\title{
LA CIRCULATION DES POISSONS MIGRATEURS : LE TRANSIT A TRAVERS LES TURBINES DES INSTALLATIONS HYDROÉLECTRIQUES
}

\author{
M. LARINIER*, J. DARTIGUELONGUE**
}

"CSP - CEMAGREF, BP 26, 31321 CASTANET-TOLOSAN CEDEX
"300 R. H-Desbals. 31100 TOULOUSE

\section{RÉSUMÉ}

La réussite des programmes de resta:.'ration des populations de poissons migrateurs (salmonidés, alose...) lancés en France ces dernières annèes nécessite que leur libre circulation soit assurée notamment au niveau des installations hydroélectriques. Lors de la migration d'avalaison, les dommages subis par les juvéniles au cours de leur passage à travers les turbines sont difficilement prévisibles et peuvent se révéler importants.

Entre 1984 et 1986, sept expérimentations ont été réalisées en France sur divers types de turbines (FRANCIS, KAPLAN, OSSBERGER), afin de mettre au point un protocole standard d'évaluation des mortalités.

Les résultats obtenus lors de ces études ont permis de compléter les données disponibles dans la bibliographie et de réaliser une analyse statistique d'ensemble. Cette analyse a montré que l'importance des dommages est étroitement liée aux caractéristiques de la turbine et à la taille des poissons.

Sur les turbines FRANCIS, la mortalitè est influencèe par des paramètres comme les vitesses absolue et relative en entrée de roue, la vitesse de rotation ainsi que par le rapport taille du poisson à l'espace inter-aubes. Sur les turbines KAPLAN, la mortalite est surtout liée au rapport de la taille du poisson à l'espace inter-pales.

Des équations de régression ont été établies qui permettent de prédire l'ordre de grandeur de la mortalité sur ces deux types de turbines pour les salmonidés et les anguillidés.

\section{THE MOVEMENT OF MIGRATORY FISH : TRANSIT THROUGH TURBINES OF HYDROELECTRIC INSTALLATIONS}

\section{ABSTRACT}

The success of plans for the restoration and enhancement of migratory fish stocks (salmonids, clupeïds...) launched in France during recent years requires that their free movement be ensured, especially at hydroelectric plant locations. The damages suffered during the downstream migration by juveniles or adults passing through a turbine cannot be easily estimated and may be important.

Between 1984 and 1986, seven experiments were carried out on several types of turbines (FRANCIS, KAPLAN, OSSBERGER) in order to define a standardized experimental procedure for estimating turbine mortalities.

The results obtained during these experiments, completed by other past studies of turbinerelated fish mortality, have allowed a statistical analysis. It resulted that the mortality rate is highly dependent on the characteristics of the turbines and the length of the fish.

Hydrodynamic factors, such as absolute and relative velocities at the vanes entrance, as well as speed of revolution and the length of the fish related to the mean distance between vanes, affect losses in FRANCIS turbines. The only factor in KAPLAN turbines is the length of the fish related to the mean distance between blades.

Models for predicting potential losses have been formulated for FRANCIS and KAPLAN runners for salmonids and eels. 


\section{INTRODUCTION}

Dans le cadre des différents programmes de restauration ou de protection des populations d'espèces migratrices - saumon, truite de mer, alose, anguille... - initiés par le Ministère de l'Environnement depuis une quinzaine d'années, et comme conséquence de la récente loi sur la pêche en eau douce de 1984, de nombreuses réalisations de passes à poissons ont vu le jour en France. Le retour d'expérience, en particulier lors du contrôle et du suivi de ces dispositifs, s'est traduit par des progrès certains dans la connaissance du comportement migratoire des espèces et dans la définition des critères de dimensionnement des ouvrages.

Il convenait d'aborder parallèlement les problèmes liés à la dévalaison des juvéniles - et des adultes pour certaines espèces _ , dévalaison qui ne se fait pas sans dommages lorsque les migrateurs sont entraînés dans les prises d'eau des centrales hydroélectriques.

Avant d'envisager systématiquement au niveau de chaque prise d'eau la mise en place de dispositifs d'évitement - barrières physiques ou comportementales -, dont la conception et la mise en œuivre sont délicates et le degré d'efficacité souvent mal connu, les probabilités d'entraînement dans la prise d'eau ainsi que le pourcentage de mortalité potentielle lors du transit dans la turbine doivent être évalués.

Les probabilités d'entraînement dans la prise d'eau sont fonction de la configuration du site, du degré d'équipement de la centrale, de l'hydrologie en période de dévalaison et du comportement migratoire de l'espèce considérée: elles ne peuvent être évaluées que cas par cas.

Le pourcentage de mortalité potentielle dans la turbine est fonction du migrateur - en particulier de l'espèce et de sa taille - des caractéristiques de la turbine et de ses conditions de fonctionnement.

On n'évoquera dans la suite que le second aspect du problème, c'est-à-dire l'évaluation des dommages potentiels lors du transit à travers une turbine, l'évaluation de la probabilité d'entraînement ne pouvant s'effectuer que ponctuellement sur chaque site.

Les expérimentations menées en France sur les dommages dans les turbines se sont déroulées entre 1984 et 1986 et ont été financées conjointement par le Ministère de l'Environnement et l'Agence Française pour la Maîtrise de l'Énergie, ainsi que par Électricité de France sur trois de ses installations.

La référence de base sur le transit à travers les turbines au moment oú était lancée l'étude était la synthèse réalisée par BELL en 1967 et actualisèe en 1981 pour le U.S. ARMY CORPS OF ENGINEERS (BELL, 1967 ; BELL et BRUYA, 1981). Dans ce rapport, l'auteur mettait en évidence l'extrême variabilité des mortalités d'une installation à l'autre en insistant sur la difficulté à dégager des modèles prédictifs des dommages potentiels; le seul moyen d'estimer raisonnablement les mortalités sur un site donné consiste alors à y effectuer une expérimentation grandeur réelle.

On s'est donc attaché à mettre au point, à l'aide de plusieurs expériences in-situ, un protocole expérimental standard susceptible d'être utilisé par la suite sur les sites présentant un intérêt particulier.

Parallèlement, les données afférentes aux expérimentations les plus intéressantes effectuées à l'étranger ont été recueillies et on a tenté de relier l'importance des dommages à certaines caractéristiques de la turbine (géométrie, champs de vitesse...).

Ces travaux ont fait l'objet, de la part de l'un des auteurs, d'une thèse de Doctorat de l'Institut National Polytechnique de Toulouse (DARTIGUELONGUE, 1988).

Après une description schématique des différents types de turbines (I), un bref historique des expérimentations menées à l'étranger est effectué (II). Les expérimentations menées dans le cadre de cette étude ont été synthétisées en III. Les essais de modélisation des dommages potentiels à partir des données françaises et étrangères ont été traités en IV.

\section{INTRODUCTION}

In the light of different programs for the restoration or protection of migratory species -salmon, sea trout, shad - initiated by the Ministry of Environment 15 years ago and as a consequence of the new legislation on Sport fishing in 1984, many fish passes have been constructed in recent years in France.

As a result of experience gained, particularly during the control of these fishways, certain advances have been made in the knowledge of the behaviour of these species and in the definition of criteria for the design of fishpasses.

At the same time, it is necessary to tackle the problems associated with the downstream migration of juveniles - and of adults for certain species - which does not take place without losses as the migrants are caught up in the intakes of hydroelectric powerstations. 
Before considering, at each intake, the installation of guiding and diverting structures physical or behavioural barriers - whose conception and setting up are critical and of which often the degree of efficiency is little known. the probability of entrainment at the intake as well as the percentage of potential biological damage during transit through the turbine, must be evaluated.

The probability of entrainment into the intake is a function of the configuration of the site, the capacity of the turbine in comparison with the river discharge during downstream migration, and the migratory behaviour of the considered species.

The percentage of potential damage in the turbine must be a function of the fish - species and length - and of the characteristics of the turbine and of its operating conditions.

Only the second aspect of the problem is considered here - that is the evaluation of potential damages during transit through a turbine since the evaluation of the probability of entrainment can only be carried out effectively at each site.

The experiments on the damages in the turbines were conducted in France between 1984 and 1986. and were financed jointly by the Ministry of Environment and the French Agency for Control of Energy (A.F.M.E.), as well as by French Electricity Board (E.D.F.) at three of the installations.

At the time the study was launched, the base reference for transit through turbines was the compendium carried out by BELL in 1967 and brought up to date for the U.S. Army Corps of Engineers (BELL, 1967; BELL and BRUYA, 1981). In this report, the author points out the extreme variability of mortality from one installation to another, stressing the difficulty of arriving at predictive models for potential damage; the only means of reasonably estimating the mortality for a given site is therefore to carry out a full scale e"periment.

It has thus been attempted to establish, with the aid of in-situ experiments, a standard procedure able to be used from now on, at sites presenting a particular interest.

Furthermore, the data pertaining to the most interesting experiments carried out abroad has been collected and an attempt has been made to relate the rate of mortality to certain characteristics of the turbines. These studies were the subject of a Doctorate thesis at the Institut National Polytechnique de Toulouse by one of the authors (DARTIGUELONGUE, 1988).

Following a schematic description of the different types of turbines (I), is a brief history of the experiments conducted abroad (II). The experiments carried out in France during this study are reviewed in 111 . The attempts at modelling the potential damages based on french and foreign data are handled in IV.

\section{I - LES TURBINES HYDRAULIQUES}

\section{GÉNÉRALITÉS SUR LES TURBINES HYDRAULIQUES}

Une turbine hydraulique est une machine tournante constituée principalement d'une roue mobile qui transforme l'énergie contenue dans l'eau d'un cours d'eau sous forme cinétique et/ou potentielle en énergie mécanique directement utilisable sur un arbre en rotation. Elle est généralement accouplée à un alternateur qui débite sur le réseau. Pour maintenir constante la fréquence, il est évidemment nécessaire de faire tourner la roue à vitesse constante.

On classe généralement les différentes turbines en deux catégories :

- les turbines à "action" (turbines PELTON, turbines BANKI-MICHELL) dans lesquelles toute l'énergie mise à disposition de la roue est sous forme d'énergie cinétique (ou de vitesse), les pressions d'entrée et de sortie de la roue étant égales à la pression atmosphérique;

- les turbines à "réaction", (turbines FRANCIS, turbines HELICES et KAPLAN), dans lesquelles l'énergie mise à disposition de la roue est en partie sous forme cinétique, en partie sous forme potentielle: l'eau subit au passage de la roue qui est sous pression à la fois une variation de vitesse et de pression.

On distingue essentiellement trois organes sur une turbine

- un distributeur, organe fixe mais souvent réglable, qui donne aux filets d'eau une vitesse, en grandeur et en direction, adéquate pour aborder la roue avec un minimum de pertes d'énergie par chocs et qui permet éventuellement de faire varier le débit admis dans la roue.

- une roue mobile, essentiellement constituée d'augets (turbines PELTON), d'aubes (turbines BANKI-MICHELL et FRANCIS), ou de pales (HELICES et KAPLAN). C'est sur l'arbre de cette roue qu'est recueillie la puissance disponible.

- un aspirateur ou diffuseur, destiné à récupérer sous forme d'énergie de pression l'énergie cinétique que peut posséder l'eau à sa sortie de la roue. Cet organe n'existe que sur les turbines à réaction 


\section{DESCRIPTION DES DIFFÉRENTS TYPES DE TURBINES}

\subsection{Les turbines à action}

\subsubsection{Les turbines PELTON}

Une turbine PELTON (fig. 1.1.) est constituée par une roue portant sur son pourtour une série d'augets en forme de double cuiller munis d'une echancrure centrale. Le distributeur se compose d'un ou plusieurs ajutages (entre 1 et 4 ) appelés injecteurs envoyant sur la roue un jet à très forte vitesse. La translation d'une aiguille à l'intérieur du distributeur permet le réglage du débit.

Le domaine d'utilisation des turbines PELTON se caractérise par des chutes généralement supérieures à $150 \mathrm{~m}$ - jusqu'à plus de $1.500 \mathrm{~m}$ - à débits relativement faibles. Le nombre d'augets est rarement supérieur à 24 .
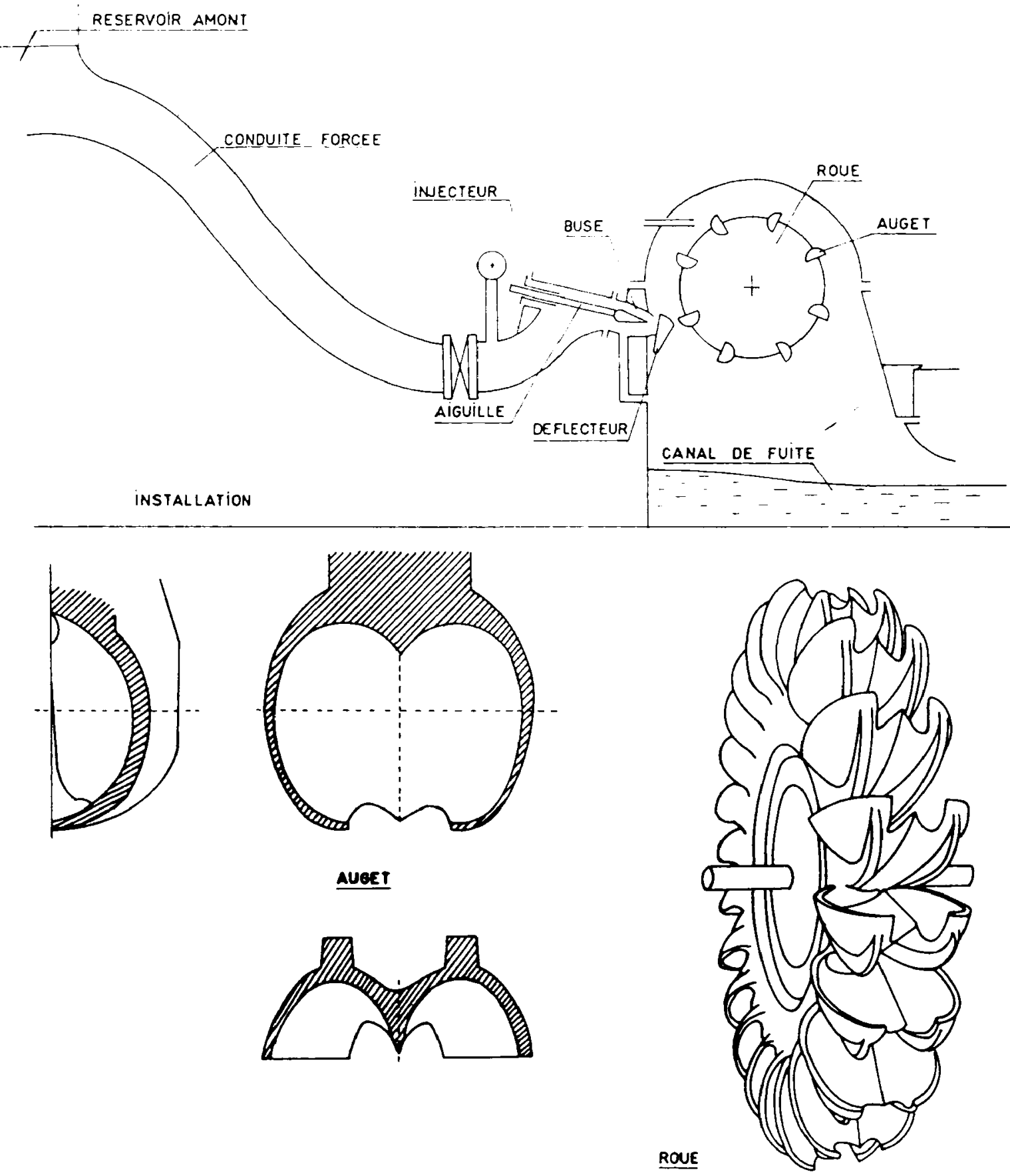

figure 1.1. : Turbine PELTON.

figure 1.1. : PELTON wheel. 


\subsubsection{Les turbines BANKI-MICHELL (OU OSSBERGER)}

Une turbine BANKI-MICHELL (fig. 1.2.) se compose d'un injecteur de section rectangulaire qui peut être modifiée par un volet profilé et d'une roue constituée de deux flasques associés par une couronne d'aubes.

Le trajet de l'eau dans la roue est à double action : l'eau agit dans un premier temps de manière centrifuge après avoir traversé la roue de l'intérieur. Il est possible de n'injecter le débit que sur une partie de la roue, ce qui permet d'accroître la plage de fonctionnement de la turbine.

Son domaine d'utilisation est assez étendu puisqu'elle peut équiper des chutes de $2 \mathrm{~m}$ à $200 \mathrm{~m}$ pour des débits variant respectivement d'une dizaine de $\mathrm{m}^{3} / \mathrm{s}$ à quelques dizaines de $1 / \mathrm{s}$. Ce type de roue est assez rarement utilisé en France.

\subsection{Les turbines à réaction}

II s'agit principalement des turbines FRANCIS et KAPLAN. Elles ont en commun les organes d'alimentation et de distribution ainsi que l'aspirateur et se distinguent surtout par la forme des roues.

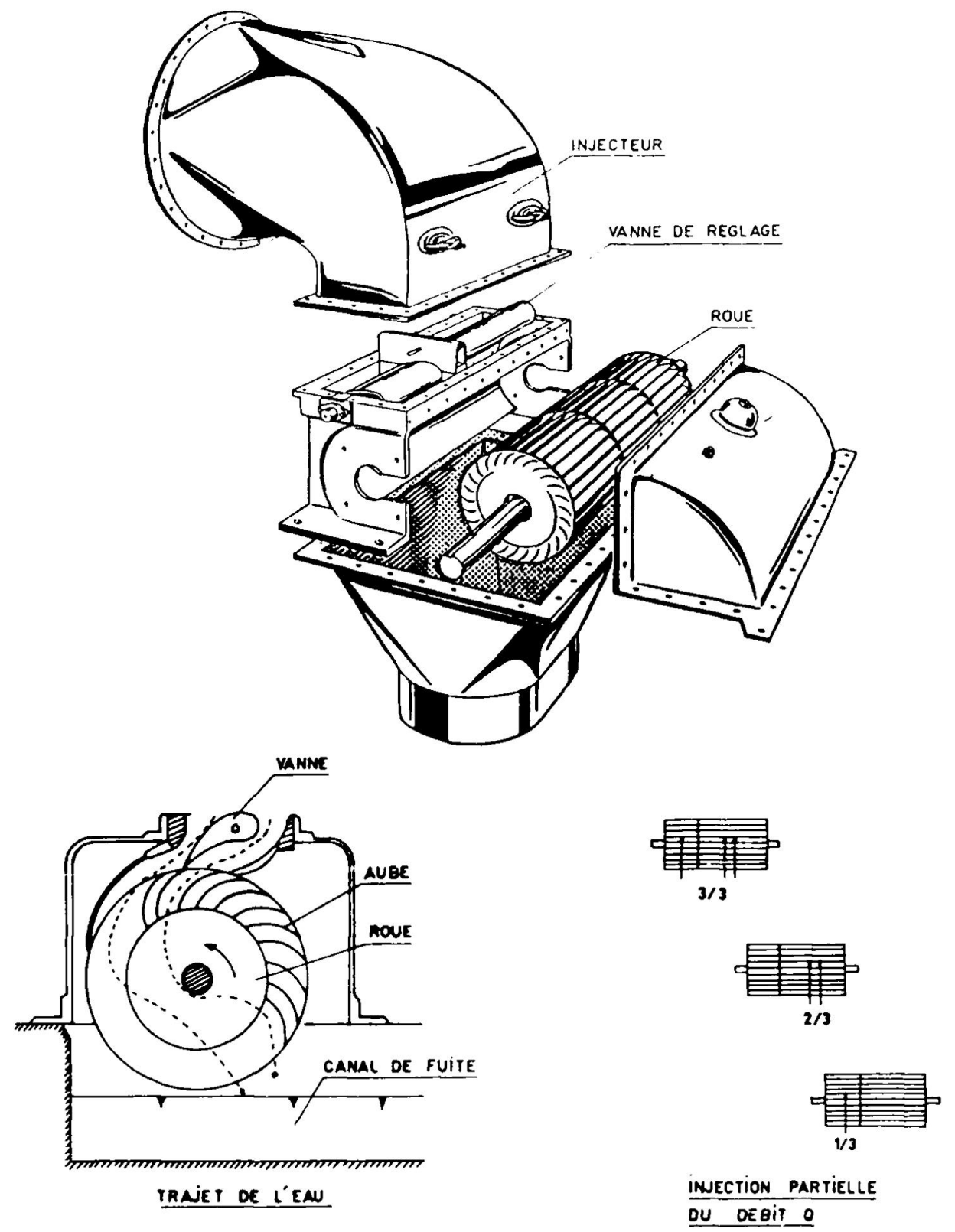

figure 1.2. : Turbine BANKI-MICHELL (ou OSSBERGER) (đoaprès documentation OSSBERGER). figure 1.2. : OSSBERGER (or BANKI-MICHELL) turbine (from OSSBERGER documentation). 


\subsubsection{Les organes d'alimentation et de distribution. L'aspirateur}

L'eau atteint la roue sur tout son pourtour apres avoir circule dans un conduit en colimaçon, la bâche spirale. Cette bâche, de section progressivement décroissante, assure une répartition constante de l'énergie à la périphérie de la roue. Sur la couronne interne sont disposées des entretoises fixes, les prédirectrices, dont le rôle est d'orienter les filets fluides vers l'entrée des directrices du distributeur (fig. 1.3.)

Le distributeur donne à l'eau une circulation convenable à l'entrée de la roue. Il est formé d'une couronne comportant un ensemble de directrices, de formes profilées, mobiles autour de tourillons parallèles à l'axe de rotation de la roue, pouvant s'ouvrir ou se fermer à la manière de persiennes. Cet organe permet de faire varier le débit admis dans la roue.

Pour les chutes en deçà de 6 mètres, la bâche spirale est gènéralement supprimée, distributeur et roue sont directement noyés dans une chambre d'eau en charge ou non.

Les turbines à réaction se terminent par un aspirateur (ou diffuseur) mettant en communication la sortie de la roue avec le bief aval. II a pour but de récupérer la hauteur de chute comprise entre la sortie de la roue el le niveau d'eau aval ainsi que la plus grande partie de l'énergie cinétique à la sortie de la roue. L'évasement très progressif de l'aspirateur est destiné à limiter la formation de tourbillons et d'instabilités inherents au ralentissement d'une veine liquide.

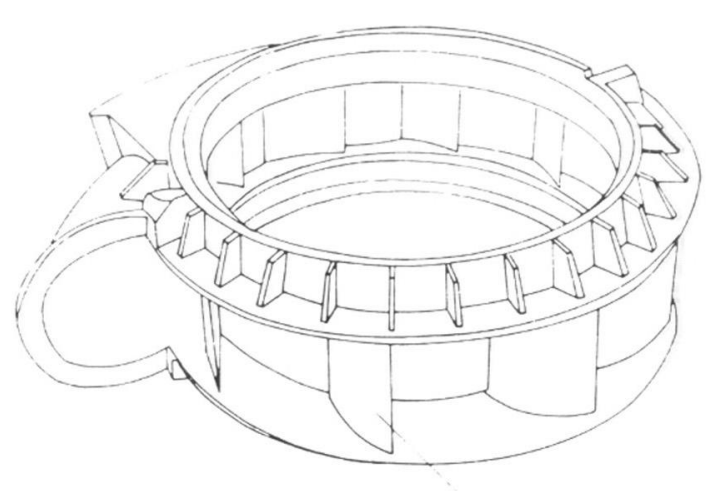

PREDIRECTRICES

COURONNE DE DIRECTRICES SUR

LA BACHE SPIRALE ( VIVIER, 1966)
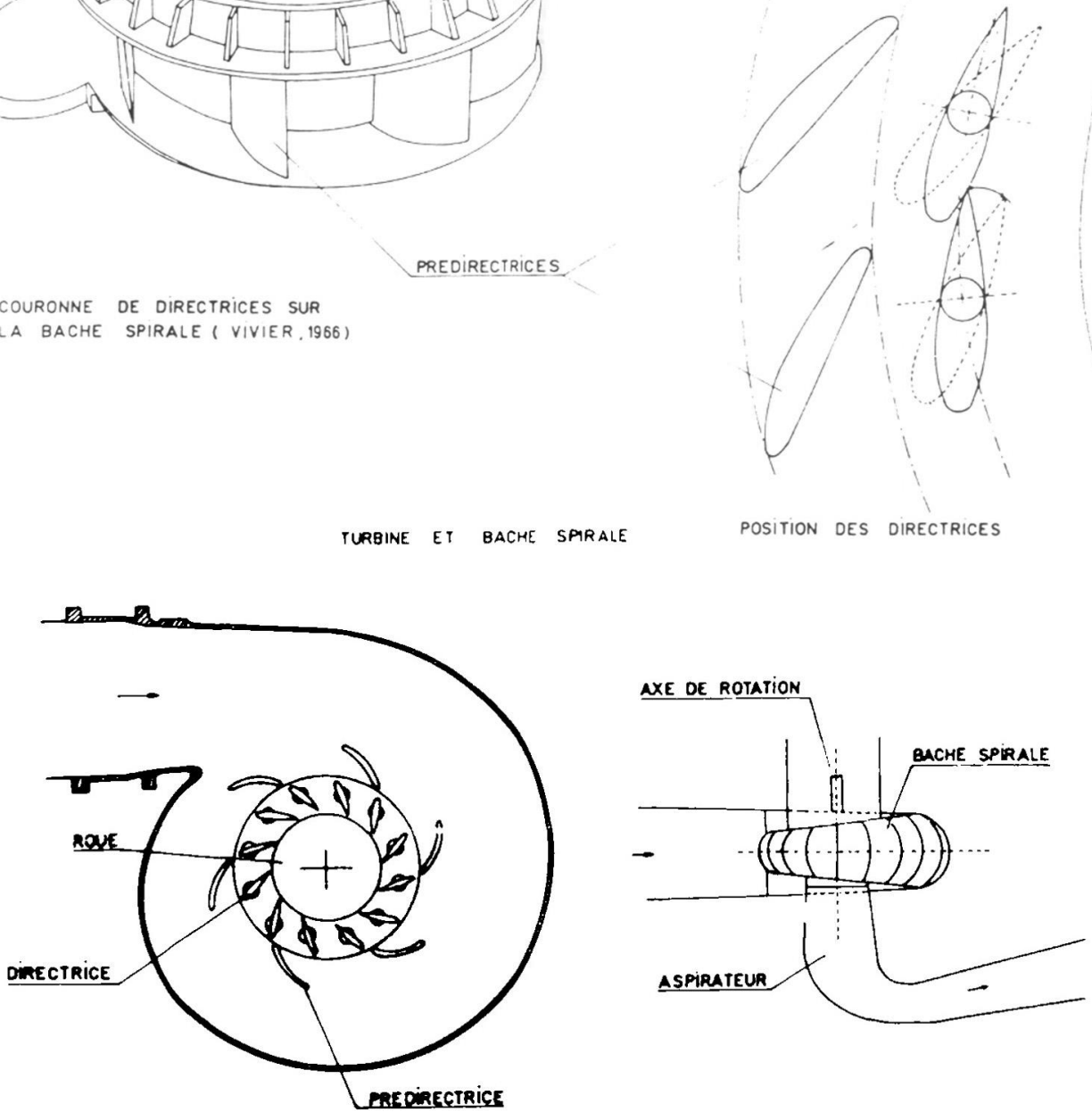

figure 1.3. : Éléments des turbines à réaction.

figure 1.3. : Elements ot reaction turbines. 


\subsubsection{Les turbines FRANCIS}

La roue (fig. 1.4.) comporte un certain nombre d'aubes - de 7 à 19 - fixèes à un arbre disposé soit horizontalement. soit verticalement. Les aubes sont des surfaces gauches donnant aux particules fluides des trajectoires absolues en forme d'hélices tracées sur des surfaces coniques. Elles sont fixees entre deux surfaces de révolution, le plafond et la ceinture. Les filets fluides qui pénètrent dans la roue dans des plans perpendiculaires à l'axe de rotation exercent une poussée sur ves aubes qui les devient et leur donnent une direction aussi voisine que possible de celle de :arbre.

La turbine FRANCIS est utilisèe dans le domaine des chutes moyennes (de 30 à plus de $300 \mathrm{~m}$ ), avec des débits allant de quelques centaines de $\mathrm{l} / \mathrm{s}$ a plus de $200 \mathrm{~m} 1 / \mathrm{s}$. Sur des installations anciennes. elles ont aussi ètè implantées sur des basses chutes.

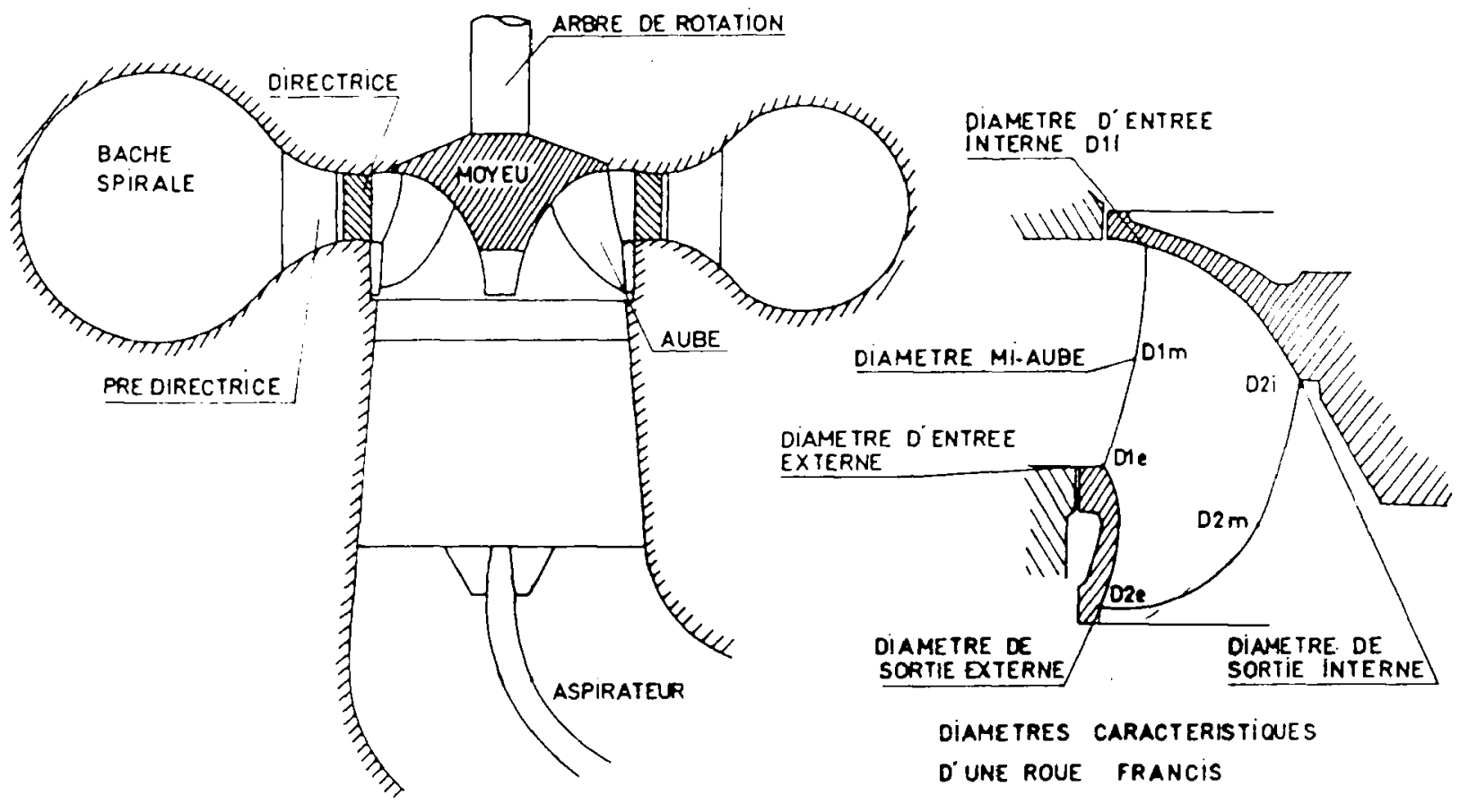

VUE GENERALE

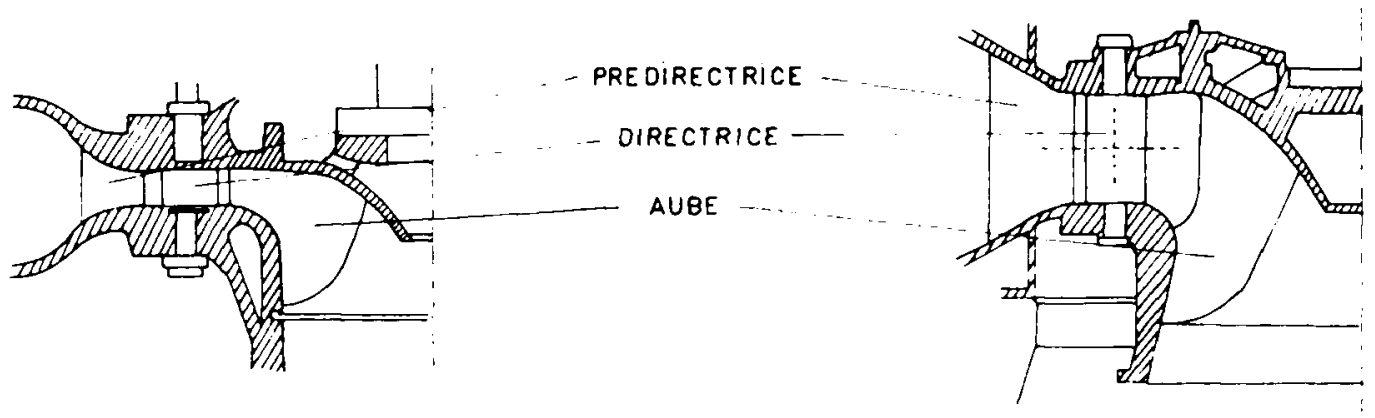

$N s=100 \mathrm{tr} / \mathrm{mn}$

Ns $=300 \mathrm{tr} / \mathrm{mn}$

EVOLUTION DU PROFIL D'UNE TURBINE EN FONCTION DE LA VITESSE SPECIFIOUE

figure 1.4. : Turbine FRANCIS.

figure 1.4. : FRANCIS turbine. 


\subsubsection{Les hélices ou turbines KAPLAN}

Les roues KAPLAN (fig. 1.5.) ne comportent que 3 à 8 pales sans couronne externe fixées sur un moyeu. Les pales sont orientables, ce qui permet un fonctionnement acceptable - c'est-à-dire avec un minimum de pertes d'énergie - pour une large gamme de débits. L'inclinaison des pales est synchronisée avec l'ouverture du distributeur.

Certaines roues KAPLAN de puissances modestes ne comportent pas de directrices, le réglage du débit et de la puissance ètant assuré uniquement par l'orientation des pales de la roue.

Une turbine hélice est une turbine KAPLAN à pales fixes. Elle ne fonctionne avec un rendement acceptable que pour une faible marge de débit.

Un groupe bulbe est une turbine KAPLAN à axe horizontal ou incliné, le distributeur et les pales pouvant être à pas fixe ou mobile ; c'est une turbine "axiale", l'axe de la turbine coincidant avec celui de l'écoulement. Ce type de turbine a permis d'abaisser le seuil de rentabilité des installations de très basses chutes en réduisant notablement le génie civil et en augmentant parallèlement l'efficacité hydraulique de l'aspirateur.

L'utilisation des turbines KAPLAN, hélices ou bulbes est réservée aux usines de basses chutes, inférieures à $30-40 \mathrm{~m}$, mais pour des débits pouvant dépasser $600 \mathrm{~m}^{3} / \mathrm{s}$.
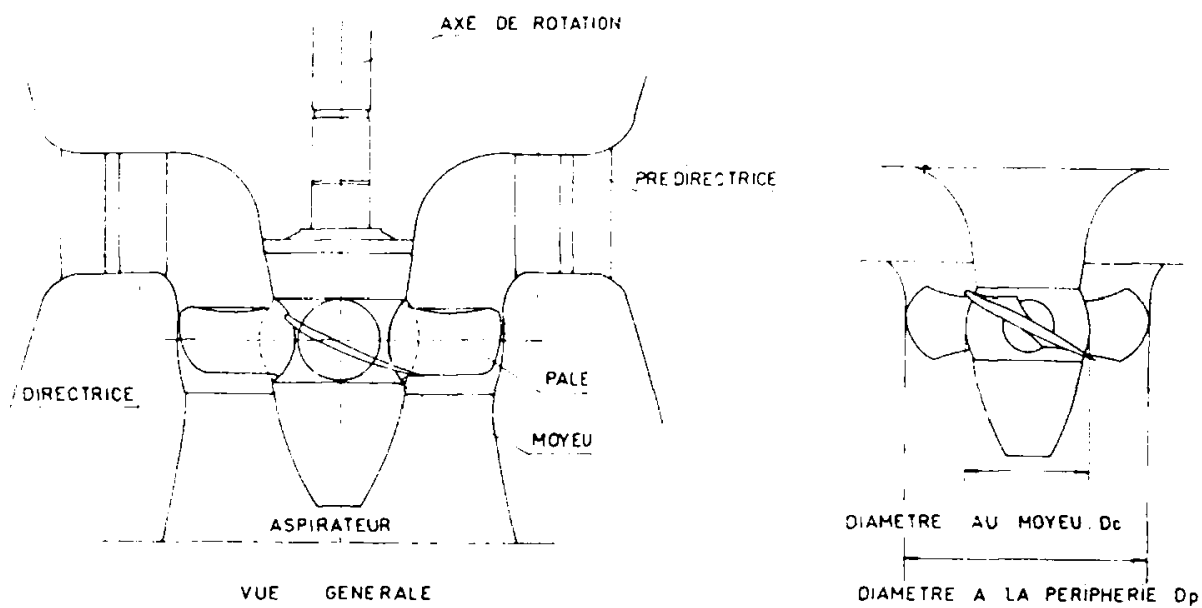

DIAMETRES CARACTERISTIOUES OUNE ROUE KAPLAN

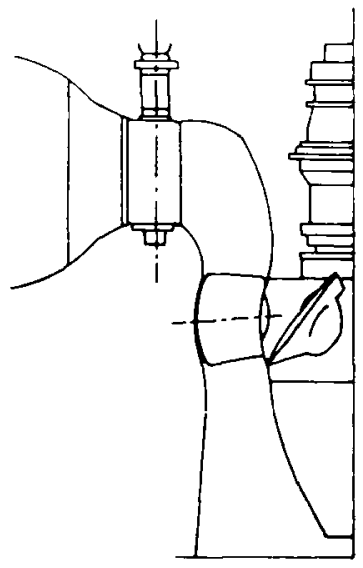

Hs: $350 \mathrm{tt} / \mathrm{mn}$

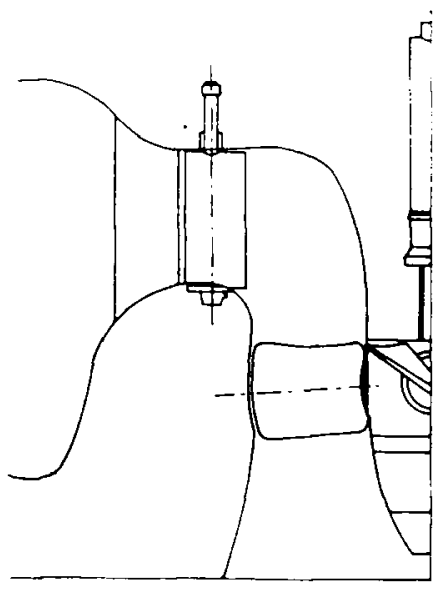

$\omega_{0}=200 \mathrm{tr} / \mathrm{mn}$

EVOUtion DU PROFIL DE LA TURAINE EN FONCTION DE LA VITESSE SPECIFIOUE

figure 1.5. : Turbine KAPLAN.

figure 1.5. : KAPLAN turbine. 


\section{NOTIONS SUR LE FONCTIONNEMENT DES TURBINES}

On ne donnera ici que les éléments essentiels à la comprèhension de la suite, notamment de la partie IV. Pour plus de détails sur le fonctionnement des turbines hydrauliques, le lecteur se reportera aux ouvrages de réfèrence sur le sujet (BROWN, 1958; VIVIER, 1966; SEDILLE, 1967; GINOCCHIO, 1978).

\subsection{Hauteur de chute et puissance nettes}

La puissance mise à disposition d'une turbine par le débit $Q$ a pour expression :

$$
\mathrm{Pn}=\text { s.g.Q.Hn }
$$

où $\mathrm{Pn}:$ puissance nette $(\mathrm{Kw})$

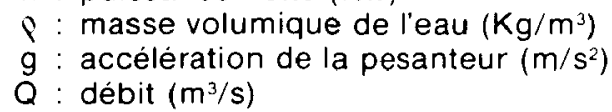

Hn est la hauteur de chute nette, c'est-à-dire la charge mise à disposition de la roue entre ses sections d'entrée et de sortie. Hn représente une énergie par kilogramme d'eau et s'exprime en mètres.

Hn est égale à la hauteur de chute brute $\mathrm{Hb}$ - différence entre la cote du plan d'eau de la prise et celle de la restitution - à laquelle on retranche les pertes de charge dans l'ouvrage de prise d'eau (en particulier aux grilles et dans les conduites ou galeries en charge situees en amont de la turbine) et dans le canal de restitution en aval de l'aspirateur.

\subsection{Les triangles de vitesse. Puissance et hauteurs de chute utiles.}

Les filets fluides pénétrant dans la roue par sa surface d'entrée subissent dans la traversée de celle-ci une déviation provoquée par les aubes. Les particules fluides sont soumises à des forces centrifuges qui, appliquées aux aubes directement ou par l'intermédiaire des couches liquides adjacentes, produisent le couple moteur sur l'axe de la rove. Le tracé des aubes est tel que ce couple soit maximal; on cherche d'autre part à rendre les plus faibles possibles les pertes d'énergie dues au frottement, à la turbulence et aux chocs.

Les particules d'eau, lorsqu'elles abordent la roue en sortie de distributeur, ont une certaine vitesse absolue (par rapport à un repère fixe, le distributeur par exemple) ; il est intéressant de définir leur vitesse relative par rapport à un repère mobile lié à cette roue. On sait que, lorsqu'un point se déplace relativement à un système en mouvement, sa vitesse absolue est la résultante de la vitesse d'entraînement du système et de la vitesse relative du point matériel. En particulier, la vitesse absolue $V$ d'une particule fluide, en un point quelconque de la roue de la turbine, sera la résultante de la vitesse d'entraînement $U$ du point lié à la roue qui se confond avec la particule considé rée et de la vitesse relative $W$ de cette particule par rapport à ce point.

On désignera par l'indice 1 (U1, V1, W1) les vitesses à l'entrée de la roue et par l'indice 2 (U2, V2, W2) les vitesses en sortie de roue.

On décompose généralement le vecteur vitesse absolue de la particule fluide $M$ suivant deux directions: sa composante giratoire $V u$, projection de $V$ sur la direction de la vitesse d'entraînement $U$ et sa composante méridienne $V m$ située dans un plan passant par l'axe de rotation de la roue (fig. 1.6.). Ces deux composantes sont importantes car nous verrons par la suite que la composante méridienne conditionne le débit alors que la composante giratoire conditionne les èchanges d'énergie.

$\mathrm{U}$

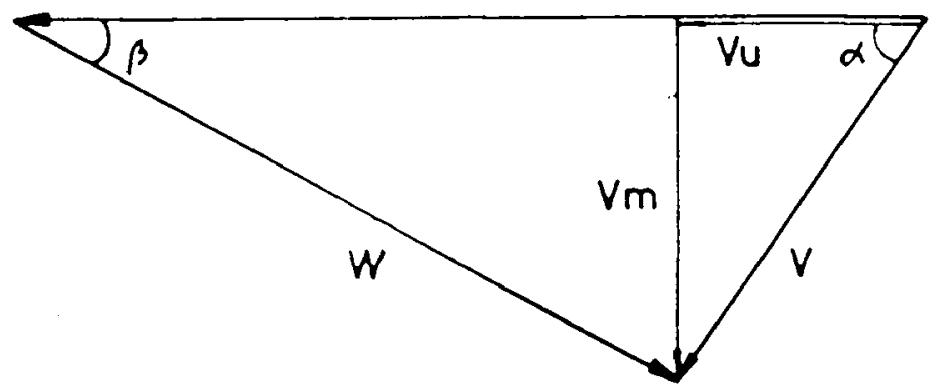

figure 1.6. : Triangle des vitesses. $V$, vitesse absolue. $U$, vitesse d'entrainement. $W$, vitesse relative. figure 1.6. : Diagram of velocities. $V$, absolute velocity. $U$, peripheral velocity. $W$, relative velocity. 
L'angle du vecteur vitesse absolue $V$ avec le vecteur vitesse d'entraînement $U$ est notè $\alpha$. En entrée de roue, il caractérise l'angle d'injection ou le degre d'ouverture du distributeur. L'angle des vecteurs vitesses relatives avec la direction négative des vecteurs vitesses d'entraînement est noté $\beta$. En entrée, il est peu différent de l'angle des aubages (fig. 1.7.). KAPLAN.

On a porté sur la figure 1.8. l'allure des triangles des vitesses pour des roues FRANCIS et

Les triangles des vitesses à l'entrée et à la sortie de la roue jouent un rôle important dans toutes les turbomachines. On montre en effet (relation d'EULER) que la puissance du couple moteur produit par l'ensemble des filets liquides - appelée puissance utile Pu - est donnée par une expression ne dépendant que des triangles des vitesses en entrée et en sortie:

$$
\mathrm{Pu}=\mathrm{P} \text {.Q. (U1.Vu1 - U2.Vu2) }
$$

Cette expression peut se mettre sous la forme d'une hauteur de chute, dite hauteur de chute effective ou utile, (Pu - P.g. Q.Hu) :

$$
\mathrm{Hu}=(\mathrm{U} 1 . \mathrm{Vu} 1-\mathrm{U} 2 . \mathrm{Vu} 2) / \mathrm{g}
$$

Hu est la hauteur de chute effectivement utilisée pour produire l'énergie mécanique sur larbre de la turbine.

La différence entre $\mathrm{Hn}$, hauteur de chute nette, et $\mathrm{Hu}$, hauteur de chute utile, est une perte de charge dite perte manométrique ou interne comprenant les pertes par frottement dans le distributeur, la roue et l'aspirateur ainsi que les pertes par choc à l'entrée de la roue se produisant lorsque la vitesse relative $W_{1}$ n'est pas tangente aux aubes en entrée.

Le rendement hydraulique in $n$ de la rove est le rapport entre hauteur de chute utile et hauteur de chute nette:

$$
\eta^{n}=\mathrm{Hu} / \mathrm{Hn}
$$

Pour donner au rendement hydraulique une valeur maximale, la vitesse de rotation de la turbine étant supposée fixée, on cherchera:

- à augmenter la composante giratoire Vu1, c'est-à-dire à diriger les filets liquides à l'entrée daris une direction aussi voisine que possible de la tangente au cercle d'entrée.

- à réduire la composante giratoire Vu2, c'est-à-dire à donner aux aubes une forme telle que les filets liquides à la sortie soient parallèles à l'axe de rotation de la roue.

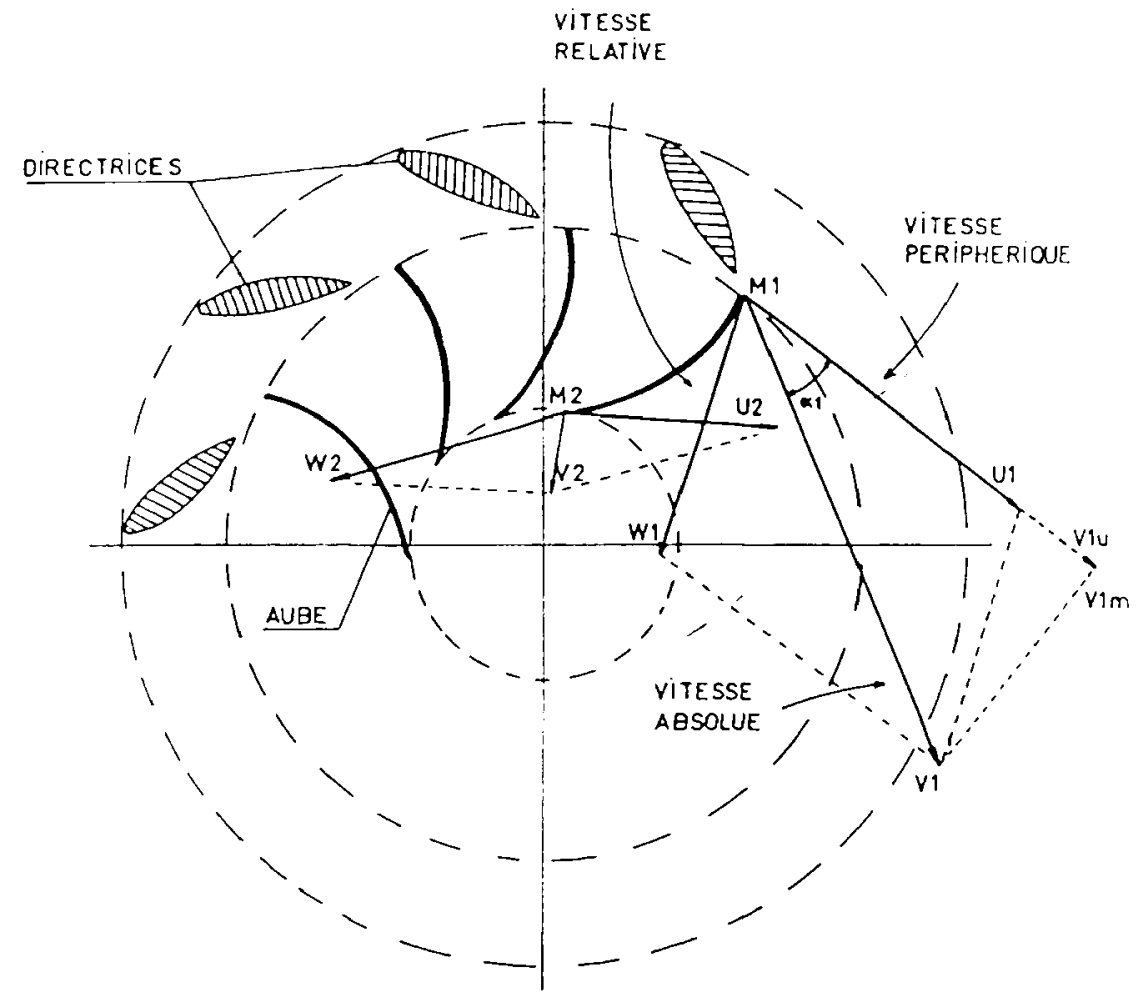

figure 1.7. : Triangles des vitesses d'une turbine à réaction en entrée et en sortie. figure 1.7. : Inlet and outlet diagrams of velocities for a reaction turbine. 


\subsection{Similitude hydraulique et vitesse spécifique}

Il est difficile de parler des turbines hydrauliques sans aborder la notion de vitesse spécifique qui résulte de l'étude des conditions de similitude des turbomachines.

De façon générale, deux systèmes mécaniques sont en similitude hydraulique s'ils sont géométriquement semblables (leurs dimensions linéaires sont proportionnelles) et cinématiquement semblables (les vitesses du fluide en des points homologues sont dans un même rapport).

Deux turbines ( $T$ ) et ( $T^{\prime}$ ) hydrauliquement semblables, de diametres $D$ et $D^{\prime}$, de vitesses de rotation $\mathrm{N}$ et $\mathrm{N}$ ', fonctionnant sous des chutes nettes $\mathrm{Hn}$ et $\mathrm{Hn}$ ', ont des triangles des vitesses semblables. Le rapport des vitesses en des points homologues est dans le rapport de la racinecarrée des chutes. Si.l est le rapport de similitude géométrique, c'est-à-dire le rapport des diamètres, on a les relations suivantes:

$$
\begin{gathered}
D / D^{\prime}=1 \\
U / U^{\prime}=(N . D) /\left(N^{\prime} . D^{\prime}\right)=V^{\prime}-W / W^{\prime}=\left(H n / H n^{\prime}\right)^{1 / 2}
\end{gathered}
$$

D'où :

$$
N / N^{\prime}=(1 / .1) \cdot\left(H n / H n^{\prime}\right)^{1 / 2}
$$

Les débits sont egaux aux vitesses multipliées par les sections de passage correspondantes

$$
\mathrm{Q} / \mathrm{Q}^{\prime}=. l^{2}(\mathrm{Hn} / \mathrm{Hn})^{\prime / 2}
$$

La similitude des triangles des vitesses entraîne l'égalité des rendements hydrauliques.

Les rendements hydrauliques étant égaux, les puissances utiles sont dans le rapport:

$$
\mathrm{Pu} / \mathrm{Pu}=(\mathrm{Q} \cdot \mathrm{Hn}) /\left(\mathrm{Q}^{\prime} \cdot \mathrm{Hn}^{\prime}\right)=. l^{2} \cdot\left(\mathrm{Hn} / \mathrm{Hn}^{\prime}\right)^{3 / 2}
$$

A partir d'une turbine donnée, on peut déterminer les caractéristiques de toute une famille hydrauliquement semblable : en particulier, une turbine déterminée (T) peut être définie en rapportant ses caractéristiques (forme, vitesse de rotation, puissance) à celles d'une turbine hydrauliquement semblable de référence produisant une puissance de $1 \mathrm{CV}$ sous 1 mètre de chute. La vitesse de rotation de cette turbine de référence qui a donc le même rendement manométrique que la turbine (T) est appelée vitesse spécifique et est notée Ns.

Elle s'exprime, en fonction des caractéristiques de la turbine ( $T$ ) par l'expression:

$$
\mathrm{Ns}=\mathrm{N} \cdot \mathrm{Pu}^{1 / 2} \cdot \mathrm{Hn}^{-5 / 4}
$$

L'expérience des constructeurs a permis de déterminer les dimensions et le tracé d'uncertain nombre de turbines fonctionnant avec le meilleur rendement possible. A partir de ces résultats, il a été possible de dresser des catalogues contenant les caractèristiques géométriques et cinématiques des turbines de référence correspondantes: chacune de ces turbines est définie par savitesse spécifique.

Inversement, lorsqu'on procède à l'établissement d'un projet d'une turbine, il suffit de calculer sa vitesse spécifique $\mathrm{Ns}$ (à partir de $\mathrm{Pu}, \mathrm{Hn}$ et $\mathrm{N}$ ) et de rechercher dans le catalogue les caractéristiques de la turbine de référence géométriquement semblable. Les dimensions de la turbine prototype sont obtenues en multipliant les dimensions de la turbine modèle par le rapport de similitude:

$$
1=\mathrm{Hn}^{3 / 4} / \mathrm{Pu}^{1 / 2}
$$

Pour chaque type de turbine ont été déterminées les limites entre lesquelles peut varier la vitesse spécifique pour que la turbine soit techniquement réalisable et fonctionne avec un rendement acceptable.

Les turbines PELTON (de 1 à 4 jets) sont utilisèes pour des vitesses spécifiques comprises entre 2 et $72 \mathrm{trs} / \mathrm{mn}$.

Le domaine d'utilisation des roues FRANCiS se situe entre 60 et $400 \mathrm{trs} / \mathrm{mn}$.

Celui des turbines KAPLAN ou HELICE entre 300 et $1.200 \mathrm{trs} / \mathrm{mn}$.

Non seulement le type, mais aussi la forme de la turbine varie avec la vitesse spécifique: Ia FRANCIS lente (c'est-à-dire à faible valeur de la vitesse spécifique) a des canaux étroits, un diamètre d'entrée supérieur au diamètre de sortie et présente un écoulement nettement centripète. L'augmentation de la vitesse spécifique se traduit par l'élargissement des canaux, l'augmentation relative du diamètre de sortie, qui devient supérieur à celui de l'entrée. l'écoulement tendant à devenir axial (fig. 1.4.). Pour les turbines KAPLAN, le nombre de pales et la grosseur relative du moyeu diminuent avec la vitesse spécifique (fig. 1.5.)

\subsection{Cavitation et hauteur de suspension}

Le phénomène de cavitation peut se produire dans une turbine si localement la pression absolue devient inférieure à la tension de vapeur d'eau (soit environ $22 \mathrm{~mm}$ de mercure ou 0,30 $\mathrm{m}$ d'eau) ; l'eau se vaporise partiellement, il se forme des bulles contenant un mélange de vapeur d'eau et de gaz dissous. Lorsque ces bulles atteignent des zones à pression plus élevée, la vapeur qu'elles contiennent se condense brutalement entraînant des implosions très violentes qui génèrent des vibrations et peuvent induire une usure accélérée des aubes de la roue. 
La cavitation peut être engendrée soit par un accroissement de vitesse se traduisant par une baisse corrélative de pression locale, soit par une réduction de pression. L'aspirateur produisant une réduction de la pression en sortie de roue favorise l'apparition de la cavitation.

La condition d'apparition de la cavitation dépend de la hauteur de suspension Hs (ou hauteur d'aspiration) de la roue, c'est-à-dire de la différence entre les cotes de la section de sortie de la roue et du niveau de l'eau dans le bief aval. Pour une roue donnée, la relation suivante donne la hauteur de suspension au-dessus de laquelle les phénomènes de cavitation sont à craindre:

$$
\mathrm{Hs} \leqslant(\mathrm{Ha}-\mathrm{Hv})-\sigma \cdot \mathrm{Hn}
$$

Ha et $\mathrm{Hv}$ sont repectivement la pression atmosphérique et la pression de vapeur saturante exprimées en mètres d'eau.

$\sigma$ appelé coefficient de cavitation (ou de Thoma) critique, est fonction de la vitesse spécifique de la roue. Il varie grosso-modo de 0.03 à 1.8 lorsque la vitesse spécifique passe de 80 (turbine FRANCIS lente) à 900 (turbine KAPLAN).

EN TREE
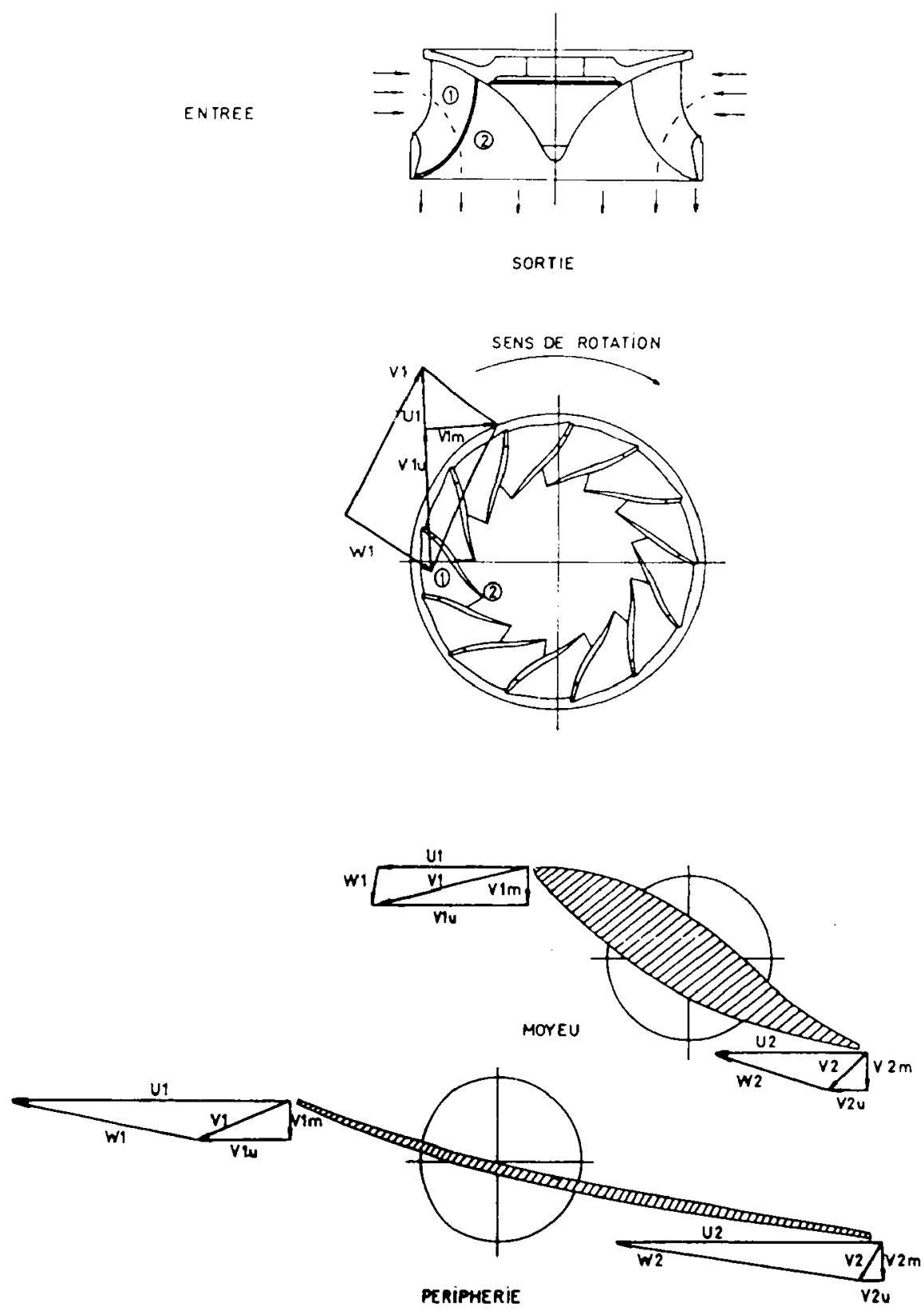

figure 1.8. : Allure des triangles des vitesses pour des turbines FRANCIS et KAPLAN.

figure 1.8. : Aspect of velocities diagrams for FRANCIS and KAPLAN turbines. 


\section{L'ENVIRONNEMENT DU POISSON LORS DE SON TRANSIT A TRAVERS UNE TURBINE}

Les variations de vitesse et de pression que subit un poisson lors de son transit dans une turbine ont été schématisées sur la figure 1.9.

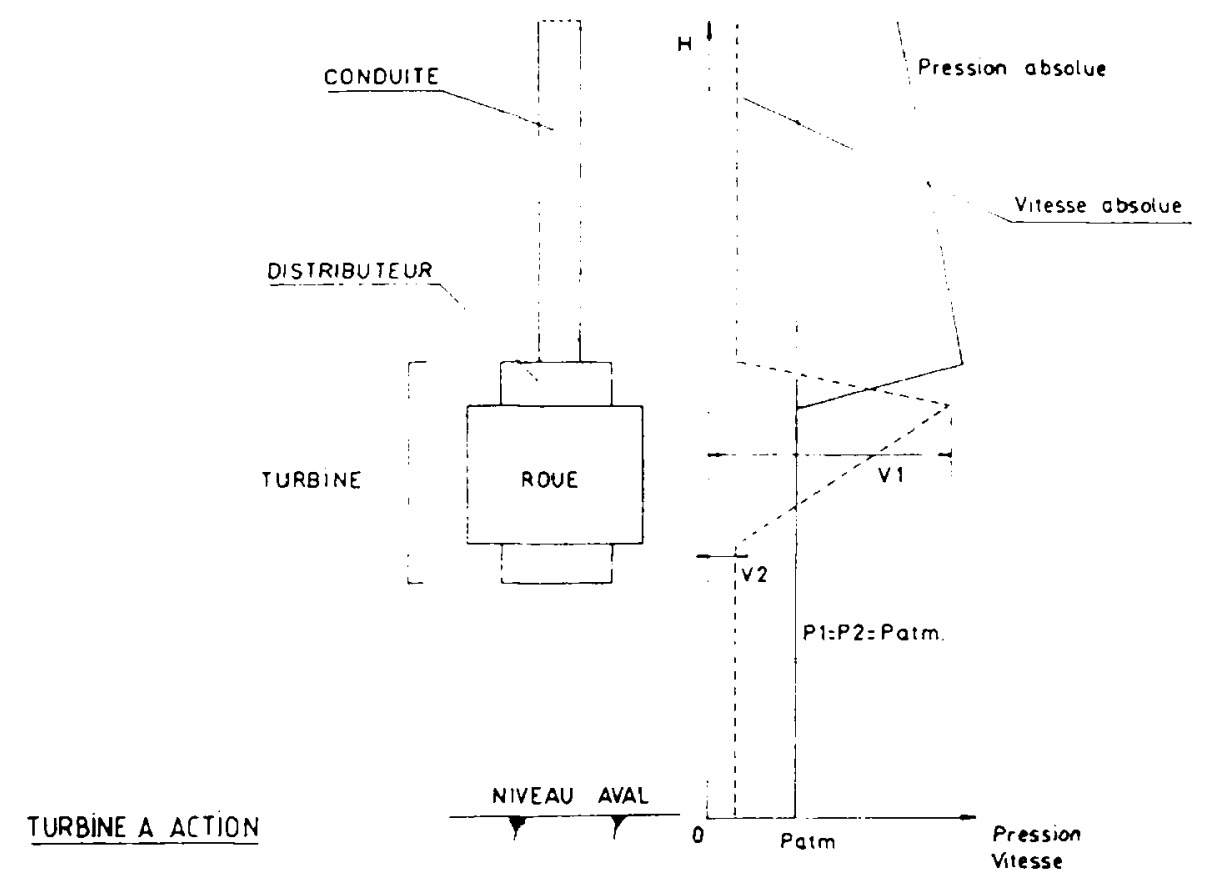

P1. pression en entrece de cove

P2, pression en sortie de rove

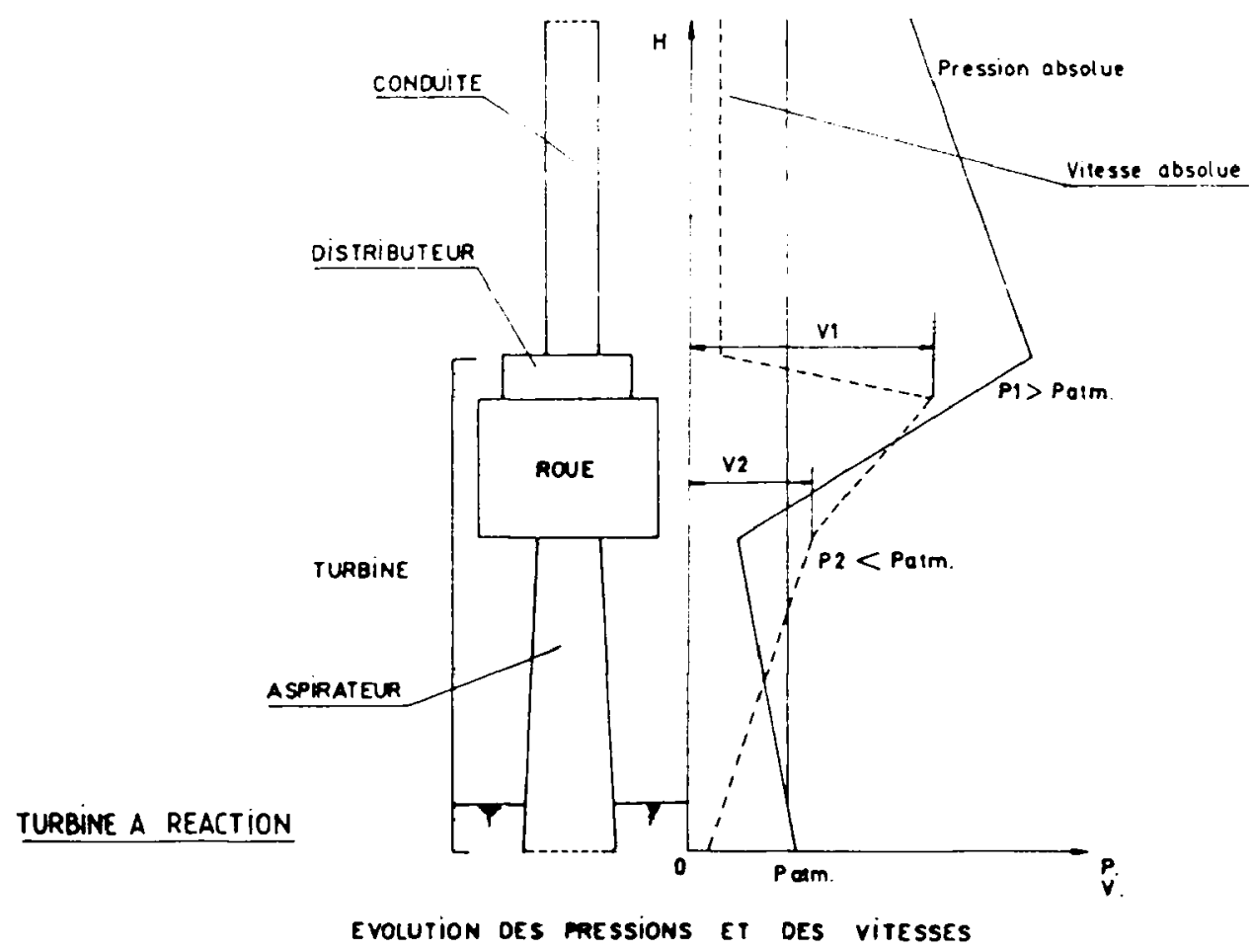

figure 1.9. : Évolution des pressions et des vitesses dans les turbines à action et à réaction. (đ̛après VIVIER, 1966).

figure 1.9. : Water pressures and flow velocities through impulse and reaction turbines. (from VIVIER, 1966). 
Les vitesses de l'eau au niveau de la prise d'eau et dans les canaux de fuite en aval de l'aspirateur de la turbine sont généralement inférieures a $2 \mathrm{~m} / \mathrm{s}$. A cette vitesse, le poisson peut éviter sans probleme les obstacles et la probabilité de mortalite est très faible. La seule possibilité de dommage se produit au niveau des grilles lorsque leur écartement ne permet pas le passage du poisson et qu'il n'existe par ailleurs aucun exutoire possible à proximité pour ce dernier: devant lutter contre des vitesses de l'ordre de $1 \mathrm{~m} / \mathrm{s}$ à $1,5 \mathrm{~m} / \mathrm{s}$, le poisson finit par s'épuiser au bout d'un certain temps et vient se plaquer et mourir sur la grille.

En aval de la prise d'eau, lors de son transit dans la turbine, le poisson est soumis à des vitesses plus fortes et à des variations de pression beaucoup plus brutales qu'il ne peut en subir dans son environnement naturel.

Il convient de distinguer les turbines à action et à réaction.

Dans les turbines à action - PELTON, par exemple -, toute l'énergie mise à disposition de la roue est sous forme d'energie cinétique, c'est-à-dire de vitesse: la vitesse du jet, voisine de $0,97 \times(2 \mathrm{~g} \mathrm{Hn})^{05}$, peut varier de $50 \mathrm{~m} / \mathrm{s}$ à près de $150 \mathrm{~m} / \mathrm{s}$ pour les chutes les plus fortes. Avec MONTEN (1985) et EICHER (EPRI, 1987), on considèrera que la probabilité de survie du poisson transitant dans une turbine PELTON est minime: même si sa taille lui permet de passer dans l'injecteur sans dommage, il ne devrait pas survivre à l'extrême brutalité des variations de pression (plusieurs dizaines de bars en une fraction de seconde) et de vitesse à l'entrée de la roue. En règle générale, les cours d'eau à grands migrateurs (saumon, alose...) ne sont fort heureusement pas concernés par ce type de turbine; par contre, le problème peut se poser pour les juveniles de truites au niveau de certains aménagements de moyenne montagne.

Dans les turbines à réaction, de type FRANCIS ou KAPLAN, que l'on rencontre fréquemment sur les cours d'eau à grands migrateurs, les vitesses sont généralement inférieures à celles atteintes dans les turbines de type PELTON. Par contre, la pression peut descendre bien en-dessous de la pression atmosphérique, en particulier en sortie de roue et dans l'aspirateur.

L'ècoulement, possédant une vitesse encore relativement modérée dans les canaux ou conduites menant à la turbine, subit une accélération au passage des prédirectrices et des directrices pour atteindre un maximum au passage de la roue, maximum pouvant se situer entre $6 \mathrm{~m} / \mathrm{s}$ et plus de $40 \mathrm{~m} / \mathrm{s}$ en fonction de la chute, de l'ouverture et du degré de réaction de la turbine. A ces vitesses, il convient de considérer le poisson comme un objet inerte, incapable d'éviter un obstacle.

Un autre paramètre important, rarement souligné par les auteurs, sauf par MONTEN (1985), est la vitesse relative de l'eau, et partant du poisson, par rapport aux aubes ou aux pales de la roue: $s i$ choc il y a, les dommages seront d'autant plus importants que la vitesse relative est forte. Dans les turbines FRANCIS, cette vitesse relative en entrée de roue peut varier suivant la chute de $4 \mathrm{~m} / \mathrm{s}$ à plus de $20 \mathrm{~m} / \mathrm{s}$ à pleine ouverture ; elle diminue cependant considérablement à ouverture partielle.

L'augmentation de la vitesse spécifique entraine celle de la vitesse relative qui devient sensiblement plus élevée que la vitesse absolue : dans les turbines KAPLAN, cette vitesse peut varier de $7 \mathrm{~m} / \mathrm{s}$ à plus de $30 \mathrm{~m} / \mathrm{s}$; elle diminue relativement peu lorsqu'on réduit l'ouverture des pales (fig. 1.10).

Il existe une autre différence fondamentale lorsqu'on considère les champs des vitesses dans les deux types de roues: dans les turbines FRANCIS, les vitesses absolues ou relatives en entree sont relativement homogènes sur toute la hauteur de l'aube (en particulier dans le cas des FRANCIS à faible vitesse spécifique), ces vitesses étant cependant susceptibles de varier considérablement suivant l'ouverture des directrices. Par contre, dans les turbines KAPLAN, les vitesses absolues en entrée augmentent de la périphérie vers le moyeu, leur rapport étant dans celui des diamètres de la roue et du moyeu ; les vitesses relatives, à l'inverse, diminuent considérablement de la périphérie au moyeu. Cette hétérogénéité des vitesses d'un point à l'autre de la roue est beaucoup plus importante que la variation de ces mêmes vitesses en un point donné résultant d'une modification des conditions de fonctionnement (ouverture ou fermeture) de la roue. On peut supposer a priori que les conditions de passage du poisson pourront être très différentes dans une roue KAPLAN suivant qu'il aborde la roue près du moyeu ou au contraire à la périphérie (fig. 1.10).

En sortie de roue, la vitesse absolue peut varier de $3 \mathrm{~m} / \mathrm{s}$ à $7 \mathrm{~m} / \mathrm{s}$ environ, pour descendre à $2 \mathrm{~m} / \mathrm{s}$ - voire moins sur les très basses chutes - en sortie d'aspirateur.

Le poisson subit une augmentation progressive de pression de la prise d'eau à l'entrée de la roue. En une fraction de seconde, au passage de la roue, il est soumis à une brutale diminution de pression, la dépression par rapport à la pression atmosphérique pouvant atteindre plusieurs mètres d'eau. Le poisson repasse progressivement à une valeur voisine de la pression atmosphérique en sortie de diffuseur. 


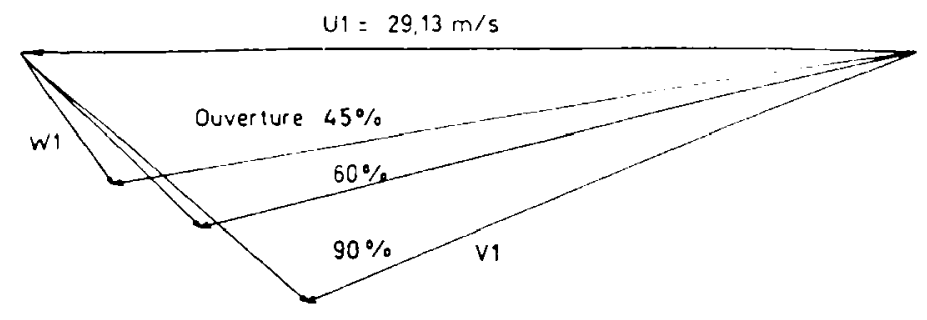

TURBINE FRANCIS DE POUTES

(CHUTE DE $61 \mathrm{~m}$. VITESSE DE ROTATION 428 TOUT $/ \mathrm{mn}$ )
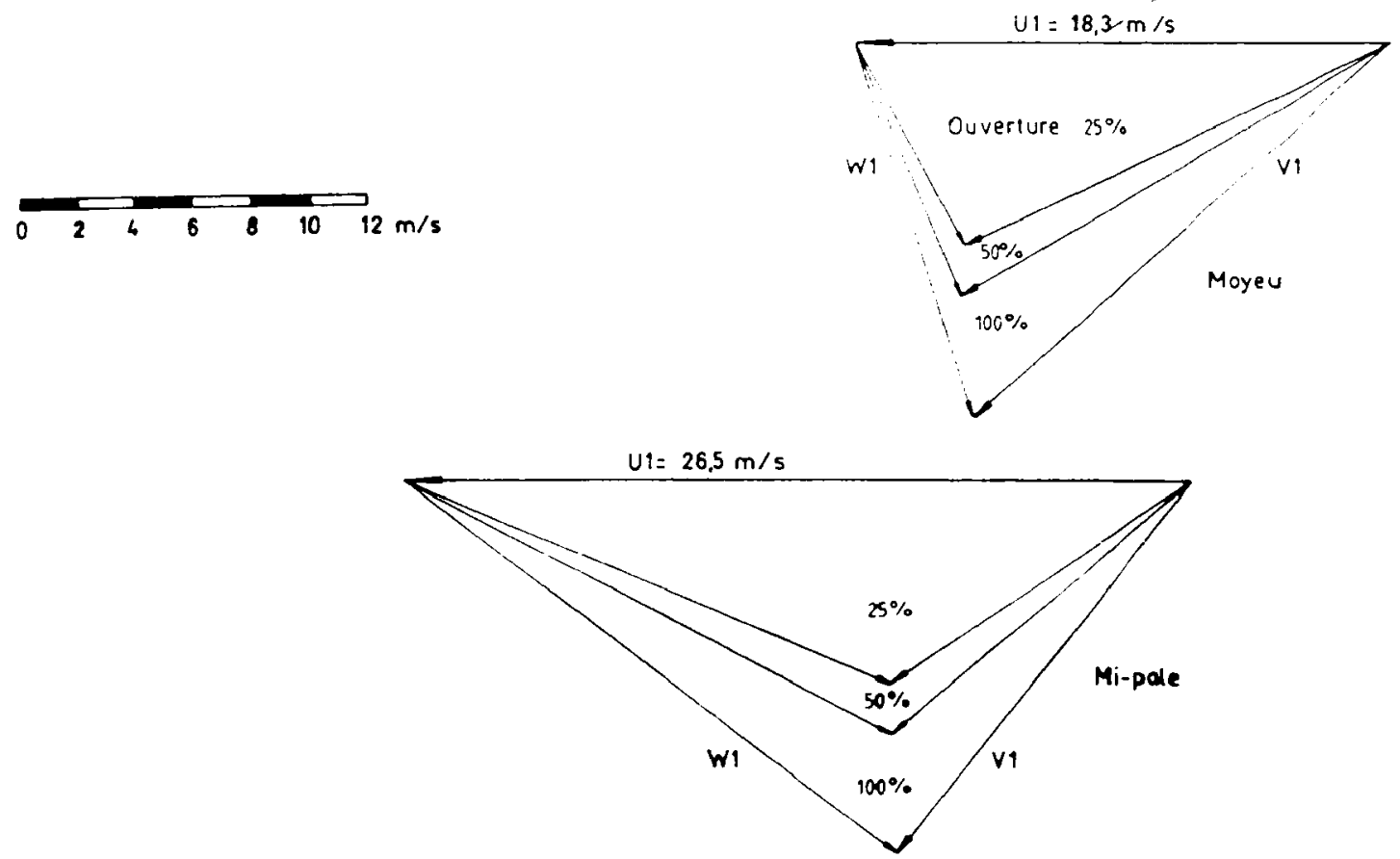

$U 1=32.8 \mathrm{~m} / \mathrm{s}$

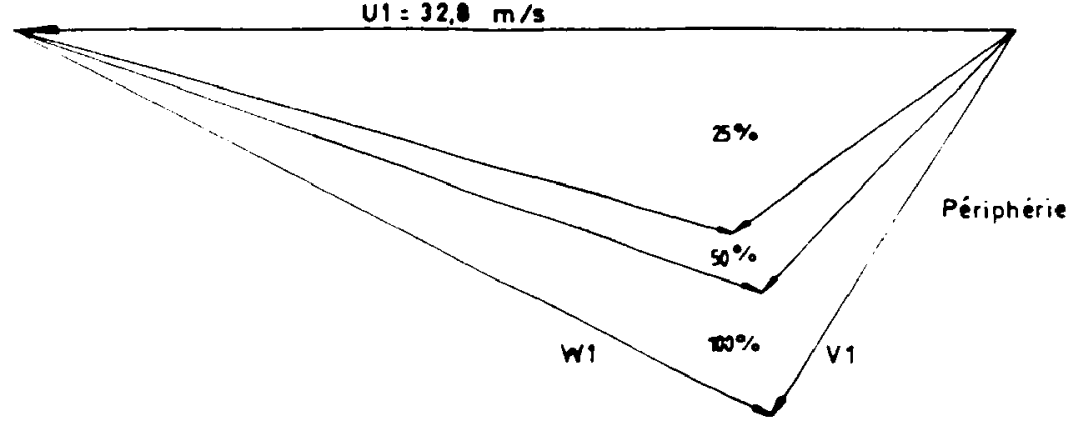

TURBINE KAPLAN DE FOSTER (d'après BELL)

(CHUTE DE $26 \mathrm{~m}$, VITESSE DE ROTATION 257 TOUIS/mn)

figure 1.10. : Évolution des vitesses en entrée de roue en fonction de l'ouverture pour des turbines FRANCIS Et KAPLAN.

figure 1.10. : Inlet velocities for various openings in FRANCIS and KAPLAN turbines. 


\section{II - REVUE BIBLIOGRAPHIQUE}

\section{HISTORIQUE}

Le problème de la libre circulation des poissons migrateurs au niveau des centrales hydroélectriques a été ressenti depuis longtemps en Europe. Les premières études théoriques ou expérimentales sur le transit des poissons dans les turbines ont vu le jour en Suede, en Allemagne et en France dans le début des années 30, menées par ALM (1927, in MONTEN, 1985), LUNDBECK (1927, in OTTERSTROM, 1931), SCHMASSMAN (1928, in KREITMANN, 1931), KREITMANN (1931) et OTTERSTROM $(1931,1932)$.

Les premières expérimentations à grande échelle ont été lancées à l'usine de Bonneville sur la rivière Columbia peu après sa construction, en 1939 (HOLMES, in LUCAS, 1962). De nombreuses autres études, toujours sur la Côte Ouest du continent Nord-Américain, leur succèdent : on citera les expérimentations effectuées sur les centrales de Baker (HAMILTON et ANDREW, 1954), d'EIwha (SCHOENEMAN et al., 1961), de Seton Creek (ANDREW et GEEN, 1958) de Cushman (CRAMER et OLIGHER, 1964), de Shasta (U.S. Army Corps of Engineers, 1963), de Big Cliff (OLIGHER et DONALDSON, 1966; U.S. Army Corps of Engineers, 1979), de Rock Island (OLSON et KACZYNSKI, 1980) et de Foster (BELL et BRUYA, 1981). Les experimentations ont porté pour la plupart sur des juvéniles de salmonidés.

Les expérimentations effectuées sur la Côte Est des États-Unis et du Canada sont beaucoup plus limitées et concernent pour la plupart des aménagements de tailles plus modestes. On mentionnera dans les provinces maritimes du Canada les essais à Tobique Narrows (Mc EARCHERN, 1960), à Tusket (SMITH, 1960, 1961), à Malay Falls (SEMPLE, 1979) et plus récemment à Lequille (Montreal Engineering Company, 1981; 1982). En Nouvelle Angleterre, le U.S. Fish and Wildlife Service a dirigé une série d'expérimentations dans le cadre des programmes de protection et de restauration des populations de certaines espèces migratrices (saumon atlantique, alose) lancés il y a une vingtaine d'années : à la centrale d'Holyoke (rivière Connecticut) avec des smolts, des juvéniles et des adultes d'aloses (KYNARD et al., 1982; BELL et KYNARD, 1985), à la centrale d'Essex (rivière Merrimack) avec des smolts (KNIGHT et KUSMESKUS, 1982) à la centrale de Colliersville (rivière Susquehanna), avec des smolts, des truitelles arc-en-ciel, des alosons et des juvéniles de bars rayés (GLOSS et WAHL, 1983)

En Europe, à part quelques expérimentations effectuées en Écosse aux aménagements d'Invergarry et de Clunie (MUNRO, 1965) et récemment en Allemagne sur la rivière Neckar (BERG, 1985), toutes les expérimentations ont été menées en Suède, par MONTEN et ses collaborateurs, notamment aux aménagements de Motala, Hemsjö, Stornorrfors, Langgó et Angabäck, plus particulièrement sur des jeunes saumons et des anguilles (MONTEN, 1985)

\section{MÉTHODOLOGIE EXPÉRIMENTALE}

\subsection{Généralités}

Plusieurs méthodes expérimentales ont été développées pour évaluer des dommages subis par les poissons lors de leur transit à travers une turbine. Ces méthodes ont évolué au fil du temps et de l'expérience: de l'évaluation à long terme à partir de techniques simples de marquage au stade juvénile et de recapture à l'état d'adulte plusieurs années après leur passage à travers la turbine, à l'évaluation immédiate à partir des techniques plus sophistiquées de radiotélémétrie.

\subsection{Estimation des mortalités juvéniles à partir du taux de retour à l'ètat adulte.}

L'une des premières méthodes utilisées pour estimer le taux de mortalitè des juvéniles lors de leur passage au travers d'une turbine a consistè à marquer différemment des lots de poissons relâcnés en amont et en aval de la turbine. Le taux de mortalité était obtenu quelques années plus tard, par différence entre les taux de retour au stade adulte de ces différents lots.

Les premières études à apporter des résultats exploitables ont été celles menées au barrage de Bonneville par HOLMES de 1938 à 1948 (EICHER, 1987). Des expérimentations basées sur le mème principe ont èté entreprises au Canada (barrage de Baker) par HAMILTON et ANDREW (1954) et en Suède à l'aménagement de Stornorrfors de 1967 à 1971 (MONTEN, 1985), aménagement dont l'importance (débit turbiné maximal de $600 \mathrm{~m}^{3} / \mathrm{s}$ ) rendait difficile le recours à des techniques plus immédiates.

Pour donner des résultats exploitables, cette méthode nécessite des taux de recapture suffisants ainsi que le marquage de plusieurs dizaines, voire centaines, de milliers de poissons.

\subsection{Estimation des mortalités à partir des recaptures immédiates du poisson}

\subsubsection{Confinement du poisson}

Afin d'accélérer l'obtention de résultats, les expérimentateurs ont cherché à recapturer les poissons immédiatement après leur passage dans la turbine. Pour faciliter leur récupération dans le 
canal de fuite, chaque poisson est enfermé dans une enveloppe ou une boîte. Cette méthode a été utilisée en Suède dans les années cinquante par CARLIN, puis par MONTEN (MONTEN, 1985) dans le but de mettre en évidence l'effet de la pression sur le poisson : celui-ci est enfermé dans une boîte en balsa; un compartiment annexe, rempli d'un lest, permet à l'ensemble d'être entraîné dans la turbine. Le lest est fixé par du papier ou de la colle hydrosoluble de telle sorte qu'il se détache de la boîte après une brève exposition à l'eau. La boîte remonte alors à la surface dans le canal de fuite où elle peut être facilement récupérée. Les taux de recapture, portant sur des lots de plusieurs centaines de poissons, ont dépassé $95 \%$.

Cette technique a été reprise et adaptée aux Etats-Unis (US Army Corps of Engineers, 1956): les poissons sont introduits dans un sac de toile attachè à deux ballons en baudruche autogonflables et un sac en papier contenant un lest. Une fois lâché, l'ensemble est entraîné dans la prise d'eau où le sac en papier, désagrégé, libère le lest. A la sortie de la turbine, les ballons gonflés font remonter l'ensemble à la surface. Sur la rivière Columbia, aux aménagements de Bonneville et $\mathrm{McNary}$, les taux de récupération sur plusieurs milliers de poissons ont été supérieurs à $80 \%$.

\subsubsection{Injection et recapture immédiate par filtration}

La méthode la plus radicale consiste à injecter le poisson à l'aide d'un tuyau le plus près possible de l'entrée de la roue et à le récupérer immédiatement à l'aval, en filtrant à l'aide d'un filet conique (fig. 2.1.) tout le débit de la turbine. Il est ainsi théoriquement possible de récupérer la totalité des poissons injectés. C'est la méthode développée en 1960 à Cushman par l'US Army Corps of Engineers et utilisée systématiquement par la suite à Shasta (1962), Big Cliff (de 1964 à 1967) et Foster (1979). Le filet est directement fixé sur l'aspirateur de la turbine; son extrémité aval conduit à un vivier supporté par un radeau dan: lequel sont récupérés les poissons (CRAMER et DONALDSON, 1964). Malgré la filtration totale du débit de la turbine, les pourcentages de recapture se révèlent assez variables (entre 45 et $95 \%$ ), les pertes étant attribuées à des déchirures du filet et au fait que dans certaines circonstances les poissons parviennent à se maintenir dans le filet, ce qui peut induire par ailleurs des mélanges entre différents lots.

Cette même méthode a été utilisée sur la Cote Est des États-Unis à la centrale de Colliersville sur la rivière Susquehanna (GLOSS et al., 1982). Elle s'est révélée satisfaisante sur les juvéniles de salmonidés; elle est par contre apparue peu adaptée aux espèces plus fragiles comme les juvéniles d'alose et de bar rayé: la mortalité attribuée à la méthode de recapture a varié de 39 à $85 \%$ pour ces espèces, comparativement à $0,5 \%$ pour les salmonidés.

Lorsque, pour une raison quelconque - importance de debit, configuration du site - la méthode ci-dessus devient difficile à mettre en œuvre, un échantillonnage plus ou moins loin à l'aval de la turbine peut être effectué (fig. 2.1.).

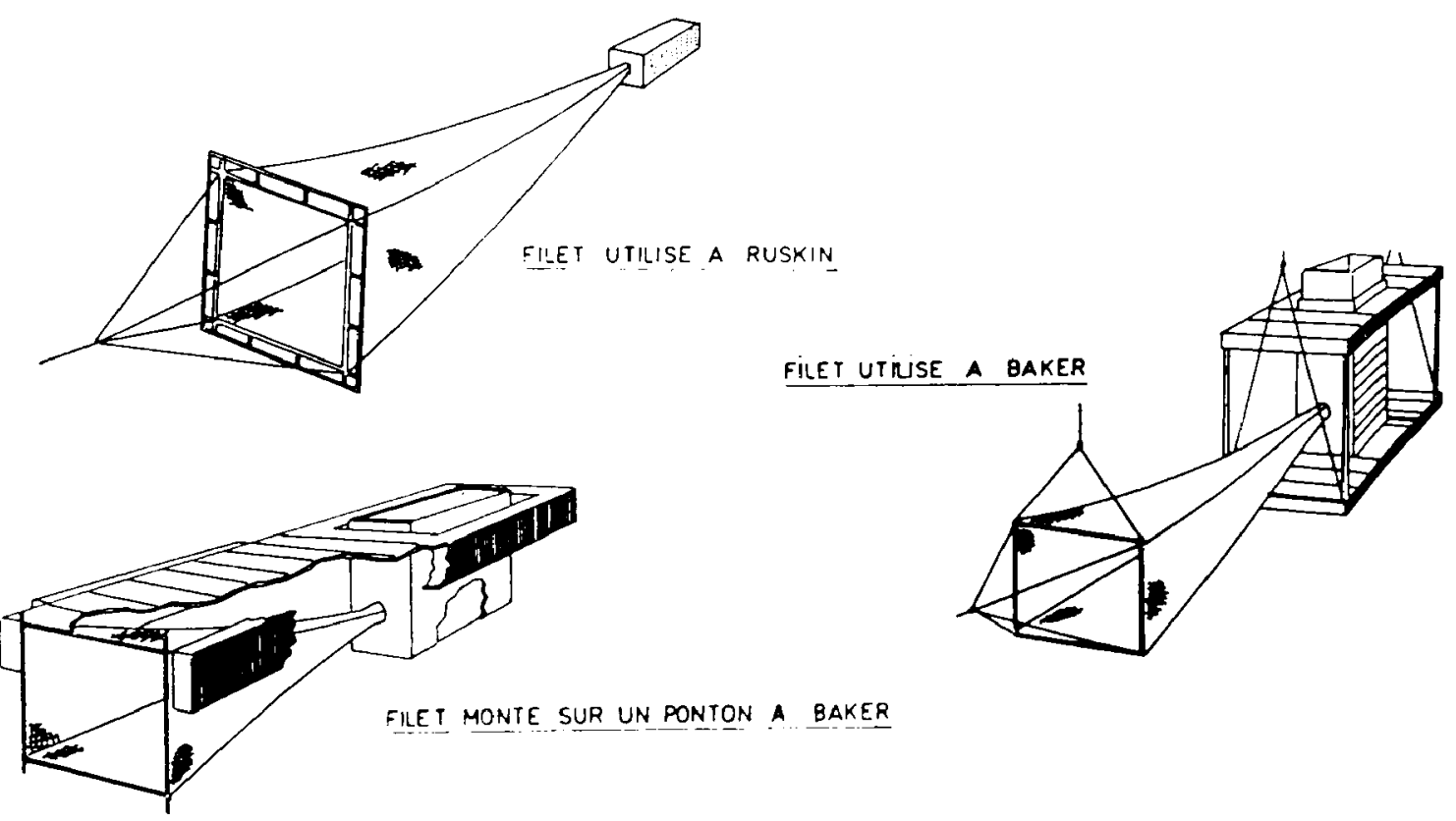

figure 2.1. : Dispositifs de récupération des poissons utilisés lors des expérimentations sur le transit des poissons à travers les turbines.

figure 2.1. : Recovery nets used tests on fish passage through hydraulic turbines. 
Au barrage de Baker, HAMILTON et ANDREW (1954) installent leur engin de pêche dans les premiers mètres du canal de fuite et parviennent ainsi à récupérer $5 \%$ des 25.000 poissons. A Big Cliff et McNary (SCHOENEMAN et al., 1954), l'échantillonnage est effectué respectivement sur près de $10 \mathrm{~km}$ et $210 \mathrm{~km}$ du point d'injection. Les taux de recapture atteignent respectivement $40 \%$ et $1 \%$, la différence s'expliquant par l'éloignement des stations d'échantillonnage à McNary et par l'étroitesse de la rivière à l'aval de Big Cliff limitant la dispersion des poissons.

Sur les aménagements des rivières Columbia et Snake, sur la Côte Ouest des Etats-Unis, une partie des juvéniles transitant au niveau des usines hydroélectriques restent piégés dans les puits de batardage des turbines. L'estimation de la mortalité subie par les juvéniles sur les installations amont peut se faire par simple échantillonnage dans ces puits. Sur 280.000 poissons lâchés dans les turbines à lce Harbor, $7 \%$ ont été récupérés dans les puits à l'usine de McNary située $72 \mathrm{~km}$ à l'aval (LONG et al., 1968). Sur 685.000 juvéniles marqués à Rock Island, près de $18 \%$ ont été récupérés au niveau des 5 installations aval (OLSON et KACZYNSKI, 1980).

\subsection{Radiotélémétrie}

La technique du radiopistage a été adaptèe récemment à l'évaluation des dommages subis par les migrateurs lors de leur passage à travers les turbines. Un émetteur radio est fixé sur le poisson qui peut être localisé et suivi après son passage dans la turbine. La miniaturisation des émetteurs rend possible leur introduction dans l'œsophage de poissons d'une vingtaine de centimètres de longueur.

Si lors des premiers essais menés au barrage d'Holyoke sur la rivière Connecticut en 1981, la mortalité sur les lots témoins de smolts a atteint 7,5\% (KYNARD et al., 1982), elle est devenue insignifiante en 1982 (STIER et KYNARD, 1986). La mortalité sur les lots témoins d'aloses adultes, ayant atteint $38 \%$ en 1981 (KYNARD et al., 1982), est passée à 1,6\% en 1982 (BELL et KYNARD, 1985).

\subsection{Conclusions}

Différentes méthodes ont été utilisées pour l'évaluation des mortalités des poissons lors de leur transit par une turbine.

- La méthode d'évaluation des taux de mortalité par comparaison des taux de retour de différents lots de poissons marqués au stade juvéniles ne demande que peu de matériel et peut être utilisée quelle que soit l'importance de l'installation. Elle permet d'intégrer l'effet du milieu sur les poissons blessés ou stressés, en particulier celui de la prédation, dont le pourcentage peut être du même ordre de grandeur que celui des mortalités directes lors du transit à travers la turbine. Cette méthode possède toutefois le double inconvénient de nécessiter un grand nombre de poissons, du fait de la faiblesse des taux de retour et de recapture des adultes, et de demander des délais très longs entre le moment du marquage et celui où il devient possible d'obtenir une estimation correcte de la mortalité.

- La méthode par confinement du poisson présente peu d'avantages : outre le fait qu'elle devient inapplicable dès que l'espacement entre aubes et directrices ou entre aubes est réduit - les boîtes en balsa sont brisées et toute récupération devient alors impossible —, il est difficile d'évaluer le biais induit par le confinement du poisson, que ce soit dans un sens ou dans l'autre.

- L'injection du poisson dans la turbine et la récupération immédiate par filtration totale du débit a généralement donné de bons résultats grâce à des taux de récupération élevés. Cependant le matériel utilisé est souvent lourd à mettre en œuvre (nappes de filet de surfaces importantes, problème de sa fixation sur l'aspirateur et de sa manipulation), l'importance du débit à filtrer étant le facteur limitant. C'est la seule méthode permettant la recapture de tous les poissons morts et partant leur examen en vue d'appréhender l'origine des dommages.

- L'évaluation de la mortalité par échantillonnage à l'aval est une opération moins lourde que la filtration totale. Cette méthode présente cependant les inconvénients propres à tout échanillIonnage: les taux de recapture souvent limités imposent l'injection d'un nombre de poissons considérable, la fraction recapturée doit être représentative de la population, et les engins de pêche exempts de toute sélectivité. Bien souvent, les auteurs ont dû recourir à des calculs correctifs plus ou moins discutables afin d'établir une estimation de la mortalité, l'efficacité de pêche étant significativement différente sur les poissons vivants et les poissons morts.

- La radiotélémétrie s'est révélée une méthode efficace, quelle que soit l'importance de l'installation. La méthodologie est simple et légère à mettre en œuvre. Elle ne nécessite pas un nombre excessif de poissons. Son inconvénient majeur réside dans un coût élevé, surtout si certains émetteurs ne peuvent pas être récupérés. 


\section{RÉSULTATS}

\subsection{Généralitès}

On peut distinguer dans la littérature deux types d'expérimentations

- celles effectuees sur un site donne dans le but d'obtenir le plus rapidement possible un ordre de grandeur des mortalités potentielles dans les conditions de fonctionnement habituelles de la roue et cela pour les especes migratrices presentes dans la rivière.

- celles, beaucoup plus approfondies et systématiques, effectuées à titre exploratoire dans le but de mettre en évidence l'influence de certains paramètres de la géométrie de la roue et de ses conditions de fonctionnement sur les mortalités et sur le type de dommage; les turbines ont alors souvent été testèes jusque dans leurs conditions extrèmes de fonctionnement.

On a repris dans les tableaux $A-I$ à $A-V I$ donnés en annexe $A$ la majorite des résultats concernant les expérimentations sur le transit des poissons dans les turbines. La plupart des données afférentes aux expérimentations effectuées en Amérique du Nord ont èté extraites des rapports de synthèse de TURBAK et al., (1981) et BELL et al.. (1967). Les données sur les experimentations suedoises ont été tirees de MONTEN (1985).

Dans les quatre premiers tableaux ont été données les caractéristiques et les conditions de fonctionnement - lorsque celles-ci sont connues - des turbines sur lesquelles ont porte les expérimentations. Dans les tableaux $A-V$ et $A-V I$ ont été reportées les données biologiques (espèces. taille, âge...) sur les poissons constituant les lots-tests, ainsi que, chaque fois quelles étaient disponibles. des indications sur la muthodologie utilisée. l'importance des échantillons et les pourcentages de récuperation.

La majoritè des expérimentations. que ce soit sur les turbines FRANCIS ou KAPLAN, ont porte sur des juvéniles de salmonidés.

Un premier examen des tableaux afférents aux turbines FRANCIS met en évidence des pourcentages de mortalité très variables: ils varient de $0 \%$ à Lower Elwha à près de $100 \%$ à Crown Zellerbach. Leur moyenne et mediane sont de l'ordre de $37 \%$, les premiers et troisièmes quartiles sont respectivement voisins de $28 \%$ et $51 \%$. Les pourcentages peuvent varier sensiblement sur une même installation, suivant les conditions de fonctionnement de la turbine.

Les dommages observés lors du transit à travers des turbines KAPLAN ou des groupes bulbes sont gènéralement inférieurs (de $0 \%$ a $30 \%$ ). Moyenne et médiane des pourcentages de mortalité sont de l'ordre de $9 \%$, les premiers et troisièmes quartiles sont respectivement voisins de $7 \%$ et $15 \%$.

Les taux de mortalités peuvent cependant devenir beaucoup plus importants sur dautres espèces, comme les clupéidés, beaucoup plus fragiles, ou les anguilles, probablement à cause de leur taille.

Lors des différentes expérimentations, les auteurs constatent généralement plusieurs types de dommages:

- les dommages dordre mécanique, causés par le contact plus ou moins violent du poisson avec un organe fixe ou mobile de la turbine.

- les dommages causés par l'exposition du poisson à des conditions de pression très rapidement variables au passage de la roue.

- les dommages attribués à l'action de cisaillement, consécutifs au passage du poisson dans des zones à turbulence extrême ou à très forts gradients de vitesse.

- les dommages attribués à la cavitation, causés par le passage du poisson dans les zones ou la pression devient inférieure à la tension de vapeur d'eau.

\subsection{Expérimentations sur modèles réduits}

\subsubsection{Expérimentation de Motala en Suède (MONTEN, 1985)}

Ces expérimentations ont été effectuées sur des modèles de roues FRANCIS et KAPLAN entre 1952 et 1954 dans le but

- de comparer les blessures infligées par ces deux types de turbines d'un diamétre identique $\left(0.60 \mathrm{~m}\right.$ ) fonctionnant à débit voisin (respectivement de 0.8 à $1.26 \mathrm{~m}^{3} / \mathrm{s}$ ) sous une même chute (14,6 mètres), les vitesses de rotation nominales étant respectivement de 381 et $598 \mathrm{trs} / \mathrm{mn}$.

- de mettre en évidence l'influence du degré d'ouverture, de la vitesse de rotation, de l'espacement entre distributeur et aubes sur la mortalite dans la turbine FRANCIS.

Les essais ont montré (fig. 2.2.) que le pourcentage de mortalité augmentait avec la taille du poisson pour les deux turbines. A taille égale. les pourcentages de mortalité étaient sensiblement moins importants sur les salmonidés que sur les autres espéces (vairons, perches). 


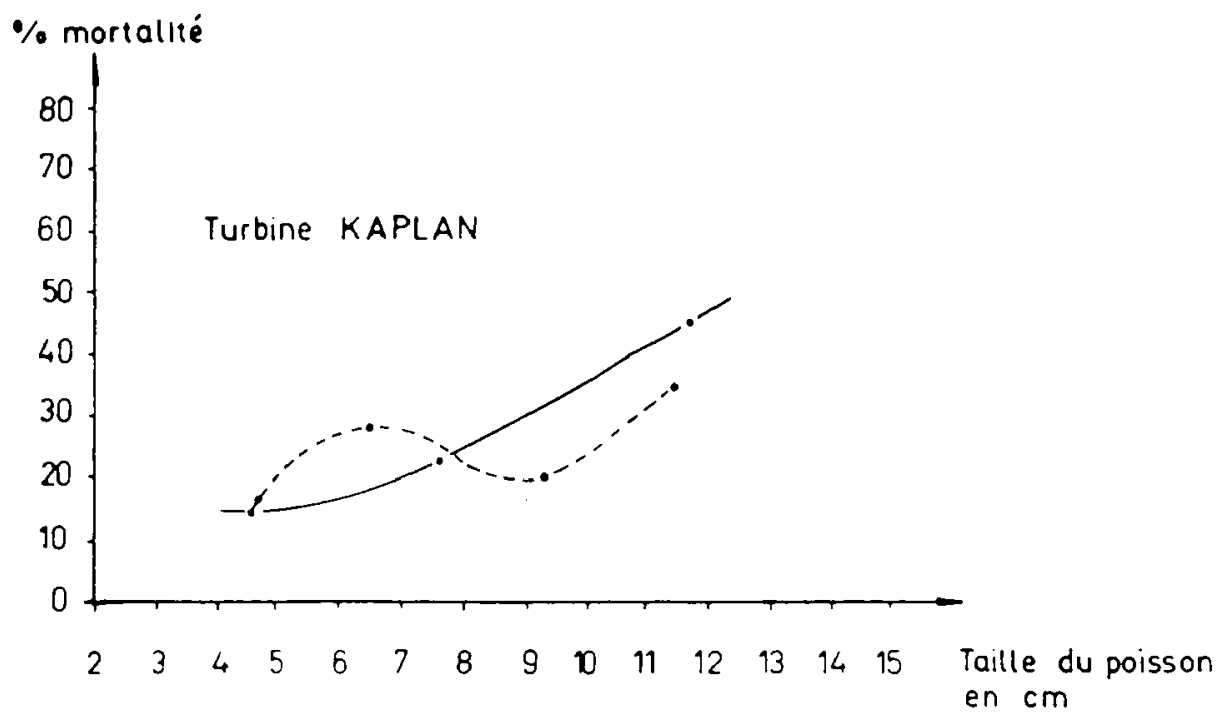

pleine charge

mi-charge

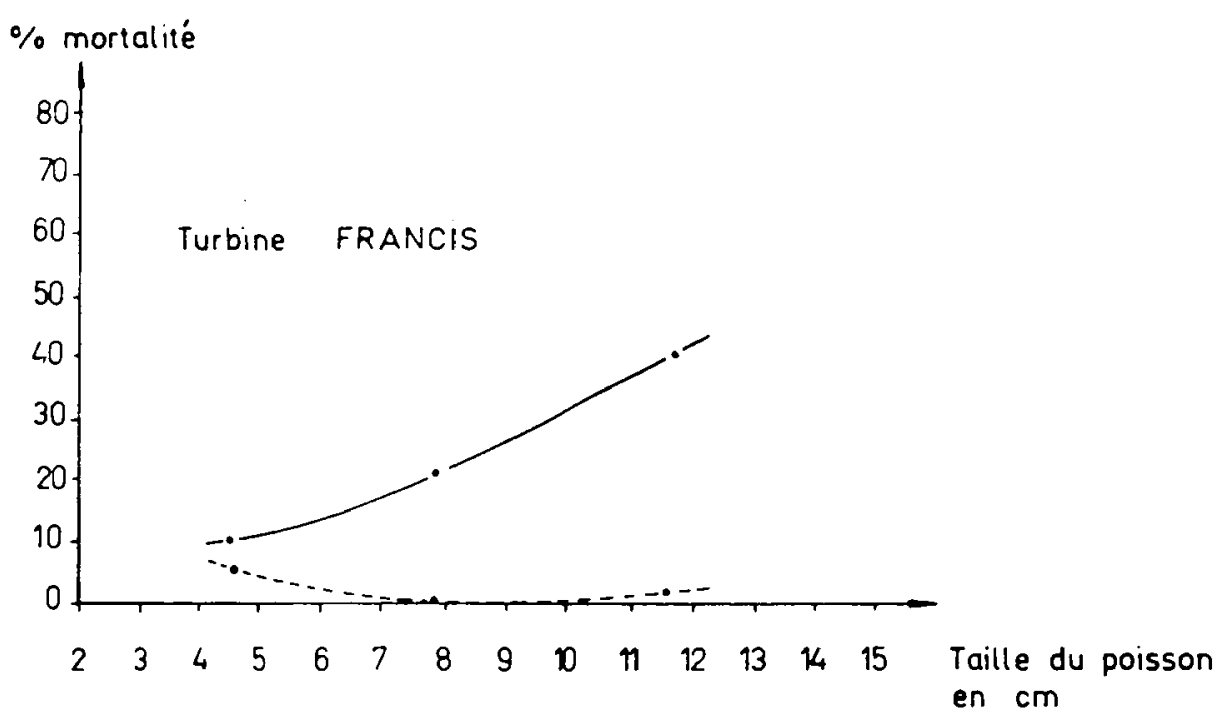

figure 2.2. : Influence de la taille du poisson et du régime de fonctionnement sur la mortalité pour deux modèles de turbines FRANCIS et KAPLAN (d'après MONTEN, 1985).

figure 2.2. : Relationship between fish size, load and losses for two model FRANCIS and KAPLAN turbines (from MONTEN, 1985).

Bien que la différence soit faible, les mortalités semblent moins importantes sur la turbine KAPLAN que sur la turbine FRANCIS, les deux turbines fonctionnant à pleine ouverture. Les types de blessure diffèrent cependant: le pourcentage de poissons coupés en deux atteint $36 \%$ sur la KAPLAN alors qu'il ne dépasse pas 1,6\% sur la FRANCIS. Par contre, le pourcentage des poissons morts sans marque apparente de blessure atteint $62 \%$ sur la FRANCIS, contre $18 \%$ sur la KAPLAN.

Les mortalités sont plus faibles à demi-ouverture qu'à pleine ouverture sur la turbine FRANCIS, alors que l'ouverture sur la KAPLAN ne semble pas affecter la mortalité.

Les résultats sur l'influence de l'espacement entre aubes et directrices sur la mortalité dans la turbine FRANCIS ne sont pas très probants dans la mesure où la vitesse de rotation n'a pas été gardée constante d'un essai à l'autre. Enfin l'influence de la vitesse de rotation n'a été mise en évidence que sur les poissons de grande taille. 


\subsubsection{Expérimentations du U.S. Army Corps of Engineers (CRAMER et OLIGHER, 1964; VON GUNTEN, 1961)}

Deux modèles réduits de turbines FRANCIS et KAPLAN de $30 \mathrm{~cm}$ de diamètre ayant respectivement 15 aubes et 6 pales ont été utilisés dans le but de mettre en évidence l'influence des conditions de fonctionnement (chute, vitesse de rotation, hauteur de suspension...) sur les mortalités de poissons de différentes espèces et tailles.

Les essais sur la roue KAPLAN ont mis en évidence :

- une augmentation de la mortalité avec la vitesse de rotation pour une chute donnée.

- une augmentation de la mortalité avec la hauteur de suspension de la roue, la chute et la vitesse de rotation restant consta.ntes.

Sur la roue FRANCIS, les essais ont montré que :

- la mortalité augmentait avec la chute, la vitesse de rotation étant conservée à sa valeur nominale (1) pour chaque chute.

- la mortalité augmentait lorsque la vitesse de rotation était abaissée au-dessous de sa valeur nominale, la charge étant conservée constante.

- quelles que soient la vitesse de rotation et la chute, la mortalité augmentait lorsqu'on fait apparaître le phénomène de cavitation en abaissant le niveau aval. Contrairement aux expérimentations suédoises, ces essais ont montre que le passage à travers la turbine FRANCIS entrain ait des mortalités légèrement moins importantes qu'à travers la roue KAPLAN, les deux turbines fonctionnant sous même chute à leur vitesse de rotation nominale. Des essais complémentaires sur la turbine FRANCIS ont mis en évidence une diminution significative de la mortalité lorsque l'espacement entre directrices et aubes était augmenté.

\section{3. Études sur prototypes de turbines FRANCIS}

On mettra l'accent sur les expérimentations au cours desquelles ont été mise en évidence des relations entre mortalités et conditions de fonctionnement de la turbine.

Entre 1960 et 1962, I'U.S. Army Corps of Engineers a effectué des expérimentations sur des jeunes salmonidés (tailles variant de 6 à $15 \mathrm{~cm}$ ) à la centrale de Cushman, dont les turbines, de $2,1 \mathrm{~m}$ de diamètre et tournant à $300 \mathrm{trs} / \mathrm{mn}$, fonctionnaient sous $140 \mathrm{~m}$ de chute (CRAMER et OLIGHER, 1964).

Les résultats ont mis en évidence une corrélation entre pourcentage de survie et rendement hydraulique de la turbine (fig. 2.3). Les dommages d'origine mécanique sont plus importants que ceux attribués à la pression, ces derniers augmentant lorsque les essais sont effectués à niveau aval bas.

Les essais sur modèle réduit ayant démontré par ailleurs l'influence de la vitesse de rotation et du clair entre aubes et directrices sur le pourcentage de survie, I' U.S. Army Corps of Engineers lança une série d'essais comparatifs à la centrale de Shasta équipée de turbines d'un diamètre plus important $(4,5 \mathrm{~m})$, fonctionnant sous une chute voisine de $130 \mathrm{~m}$ et tournant à $138,5 \mathrm{trs} / \mathrm{mn}$ (U.S. Army Corps of Engineers, 1963).

Comme il était prévisible, les pourcentages de survie se sont révélés plus importants à Shasta qu'à Cushman. Les tests ont aussi mis en évidence une similitude des courbes de pourcentage de survie et de rendement (fig. 2.3.).

De ces deux séries d'expérimentations, les auteurs ont conclu que pour minimiser les dommages sur une roue FRANCIS, il convenait d'adopter dans la mesure du possible:

- des vitesses de rotation faibles

- des rendements hydrauliques optimaux

- un calage de la turbine tel que les dépressions en sortie soient minimisées

- des espacements entre directrices et aubes maximaux.

A l'usine de Malay Falls au Canada, équipée d'une turbine de 1,66 m de diamètre fonctionnant sous $12 \mathrm{~m}$ de chute, aucune relation significative entre taux de mortalité et ouverture n'a été mise en évidence (SEMPLE, 1979). A l'usine d'Upper-Hemjö, en Suède (MONTEN, 1985), équipée d'une petite roue FRANCIS de $0,97 \mathrm{~m}$ de diamètre fonctionnant sous $16 \mathrm{~m}$ de chute, les mortalités diminuent très fortement à ouverture réduite.

(1) Pour chaque chute, la vitesse de rotation était modifiée de telle sorte que la vitesse spécifique Ns reste constante. 


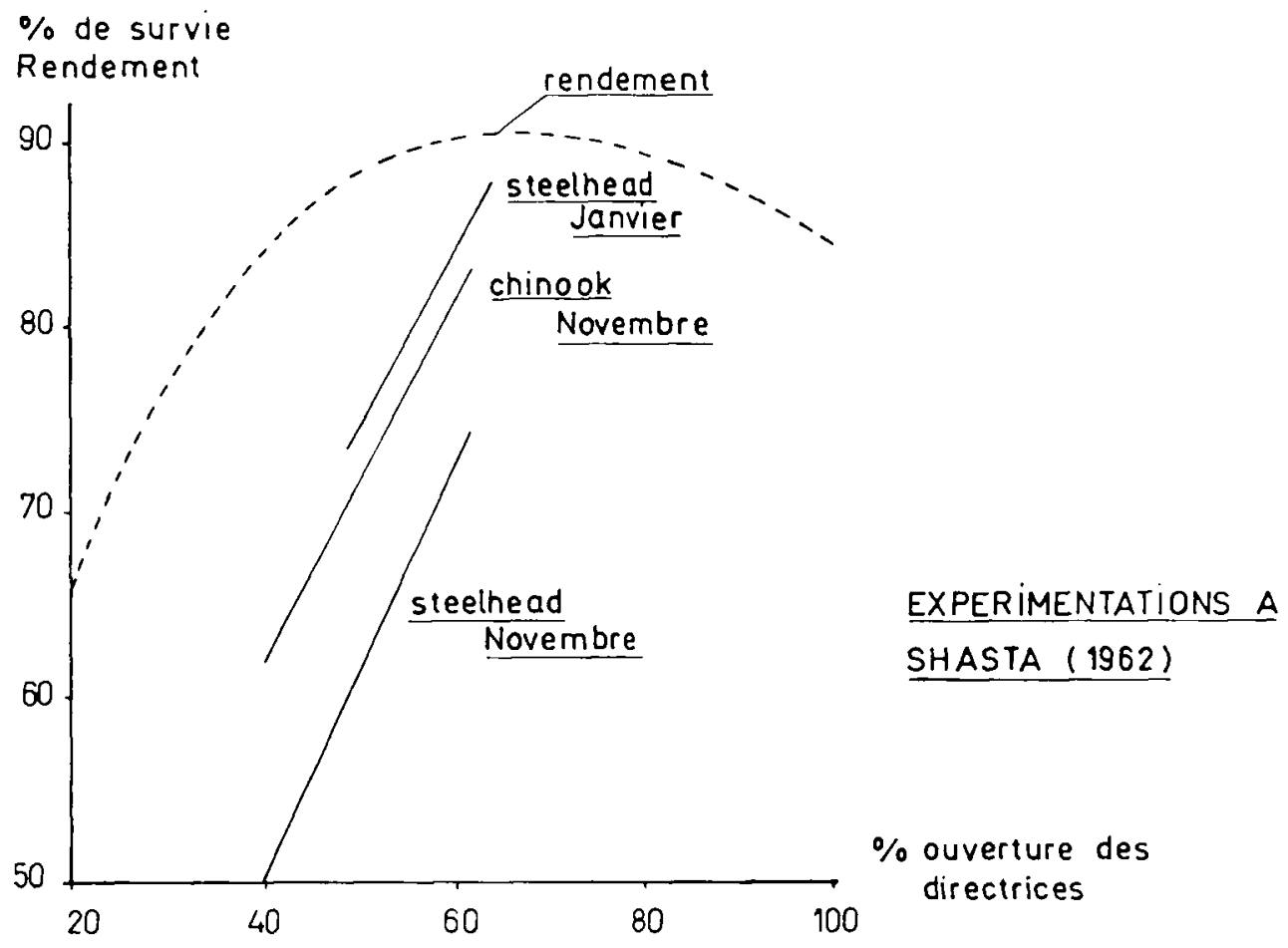

$\%$ de survie

Rendement

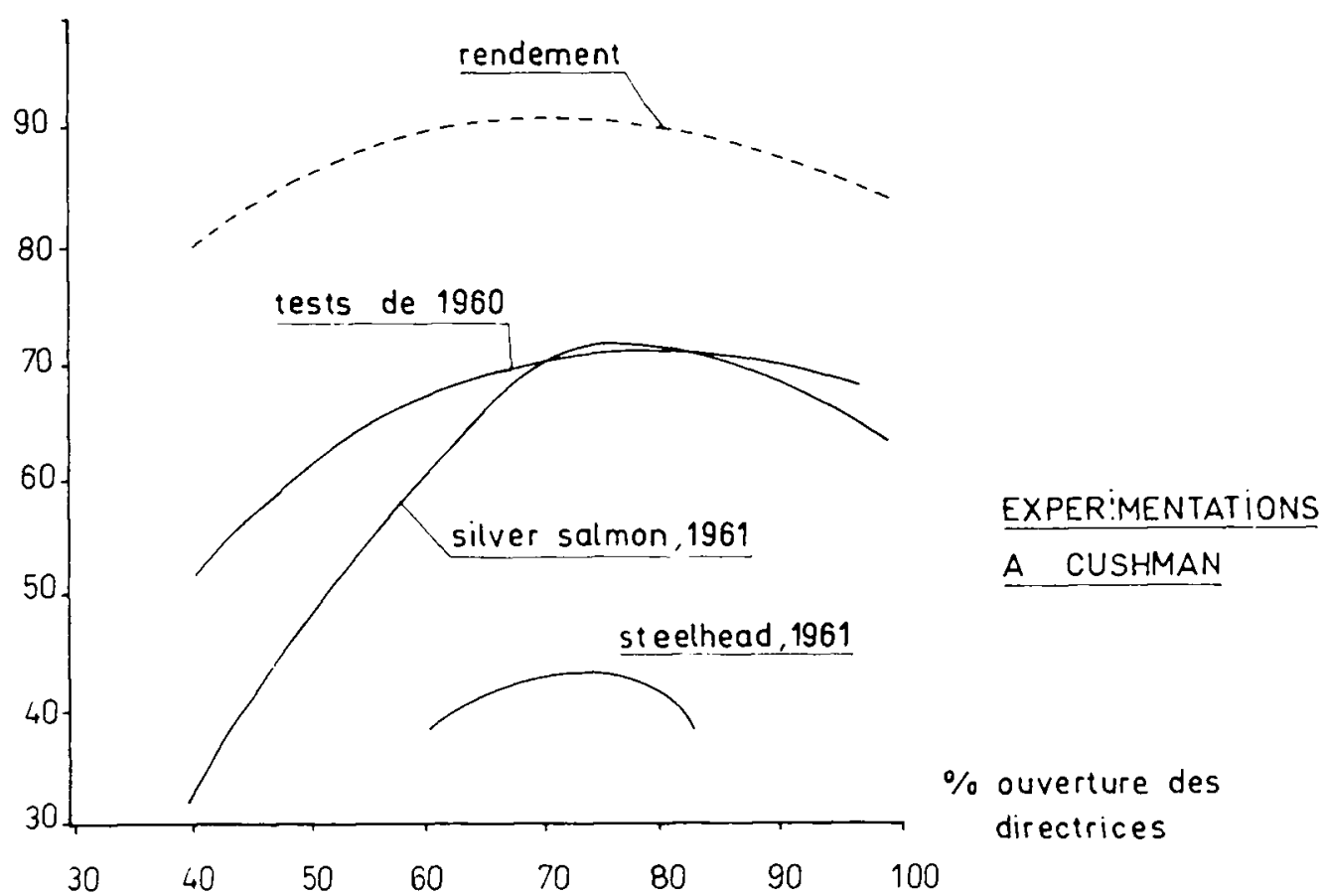

figure 2.3. : Taux de survie des poissons en fonction du rendement de la furbine aux aménagements de Cushman et Shasta (d'après CRAMER et OLIGHER, 1964).

figure 2.3. : Relationship of fish survival to turbine efficiency, Cushman and Shasta hydroelectric projects (from CRAMER and OLIGHER, 1964). 
Les expérimentations les plus fines ont été effectuées par RUGGLES et COLLINS (Montreal Engineering Company Limited, 1381 et 1982) à la centrale de Lequille en Nouvelle Écosse, équipée d'une turbine d'un diamètre voisin de $1,38 \mathrm{~m}$ tournant à $514 \mathrm{tr} / \mathrm{mn}$ sous une chute moyenne de 118 mètres. Les essais ont montré que la mortalité - variant de 15 à $76 \%$ - était avant tout liée à la taille du poisson (fig. 2.4.) ; l'influence des conditions de fonctionnement (degré d'ouverture des directrices, injection ou non d'air en sortie de roue, etc...) ne paraît pas très significative. Cependant, comme dans les deux expérimentations de Cushman et Shasta, le pourcentage de survie maximal se produit au voisinage du point de rendement maximal.

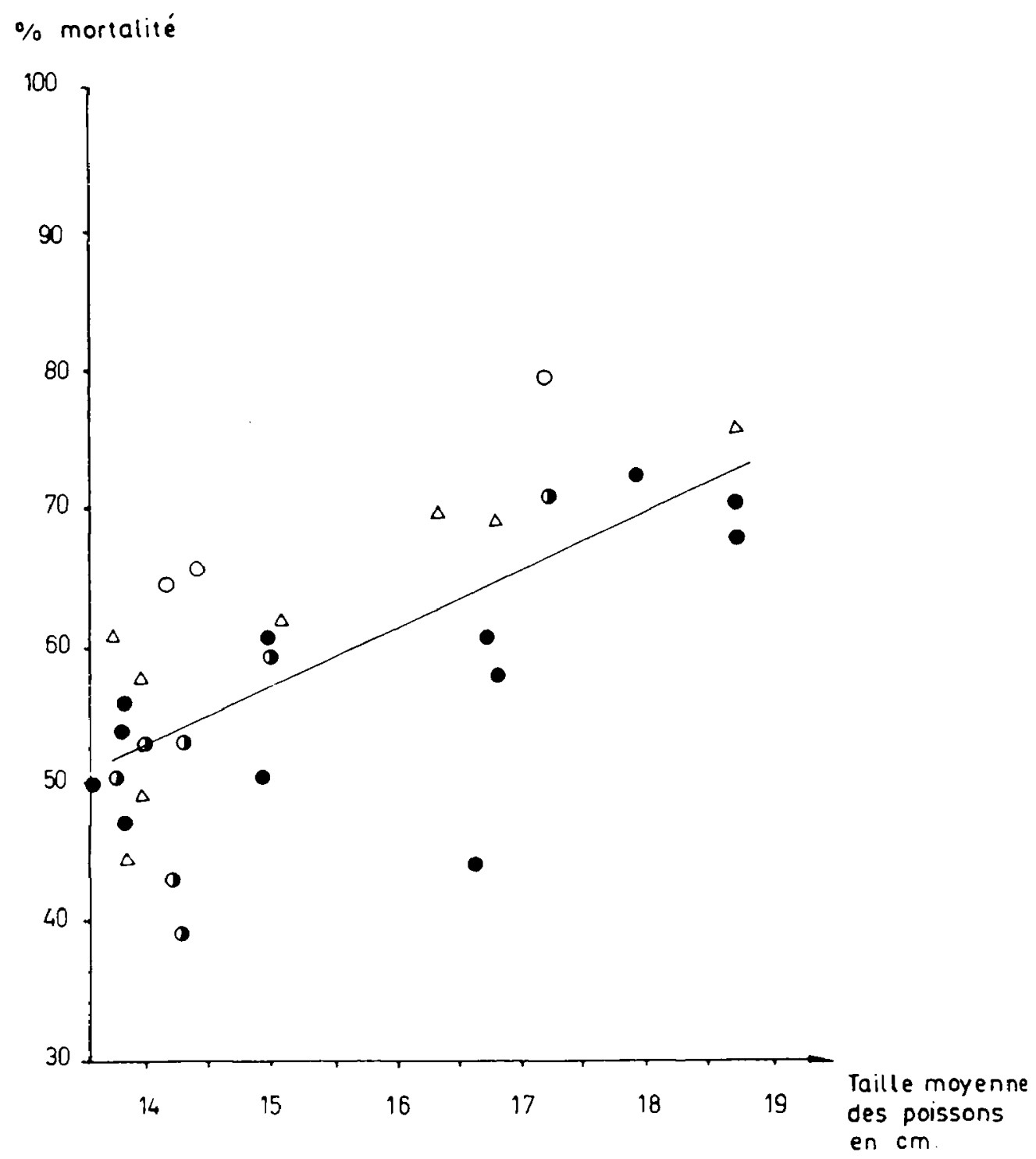
$\Delta 100 \%$ d'ouverture des directrices
- $75 \%$
- $50 \%$
o $20 \%$

figure 2.4. : Taux de mortalité des juvéniles de saumons en fonction de leur taille pour les différentes ouvertures testées à Lequille (d'après Montreal Engineering Company Ltd., 1981).

figure 2.4. : Relationship of Salmon mortality to mean length at each gate opening tested, Lequille (from Montreal Engineering Company Ltd., 1981). 


\section{4. Études sur des prototypes de turbines KAPLAN et BULBE}

Les expérimentations de référence sur le transit à travers les turbines KAPLAN sont celles effectuées par SCHOENEMAN de 1955 à 1957 (SCHOENEMAN et al., 1961) à McNary et Big Cliff sur la Côte Ouest des États-Unis. Ces turbines équipent des chutes voisines de $30 \mathrm{~m}$, et ont pour diamètres respectifs $7,10 \mathrm{~m}$ et $3,68 \mathrm{~m}$, les vitesses de rotation étant de $86 \mathrm{trs} / \mathrm{mn}$ et $164 \mathrm{trs} / \mathrm{mn}$. Aucune différence significative sur la mortalité ( $11 \%$ en moyenne) n'a été mise en évidence entre les deux turbines fonctionnant à même ouverture. A Big Cliff, la mortalité s'est révélée plus importante $(21 \%)$ à ouverture réduite des directrices $(40 \%)$ qu'à $75-80 \%$ d'ouverture $(9 \%)$. SCHOENEMAN suggère que cette différence est due à l'augmentation de la cavitation consécutive à la fermeture des directrices.

Les essais se poursuivirent à Big Cliff plusieurs années (OLIGHER et DOLNALSON, 1966 U.S. Army Corps of Engineers, 1979). Les tests ont été effectués à plusieurs ouvertures du point d'apparition de la cavitation à la pleine charge et cela pour trois hauteurs de chute. Comme pour les turbines FRANCIS, le pourcentage de survie maximal correspond pratiquement aux conditions de rendement hydraulique optimal de la turbine (fig. 2.5.).

Suite aux essais de Big Cliff, des expérimentations furent effectuées à la centrale de Foster équipée d'une roue de $2,5 \mathrm{~m}$ de diamètre, tournant à $257 \mathrm{trs} / \mathrm{mn}$ sous une chute voisine de $30 \mathrm{~m}$ (BELL et BRUYA, 1981). Les essais consistèrent à faire fonctionner la turbine, pour une ouverture de pale donnée, à ouvertures de directrices variables et cela pour plusieurs chutes. Les résultats montrent qu'à ouverture de pale donnée, la courbe de survie suit la courbe de rendement. Le taux maximal de survie, quelle que soit la chute, est obtenu avec un degré d'ouverture des pales de $25 \%$, correspondant à un angle de calage des pales de $15^{\circ}$. Ni l'effet des variations de pression au passage de la roue, ni l'effet de la cavitation n'ont pu être mis en évidence de façon très nette.

\section{$\%$ survie et rendement}

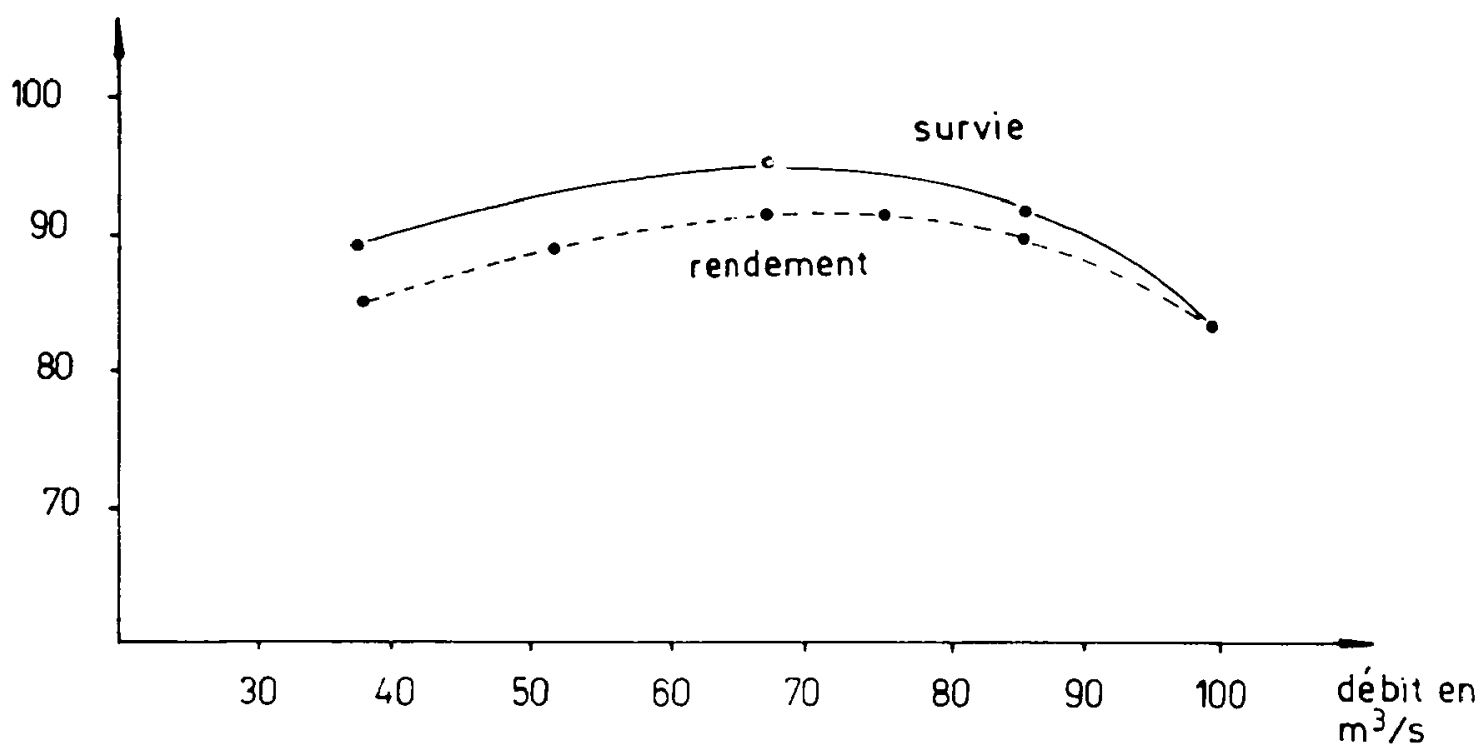

figure 2.5. : Taux de survie des poissons en fonction du rendement de la turbine à l'amènagement de Big Cliff (d'après OLIGHER et DONALDSON, 1966).

figure 2.5. : Relationship of fish survival to turbine efficiency, Big Cliff hydroelectric project (from OLIGHER and DONALDSON, 1966).

\subsection{Expérimentations sur d'autres types de turbines}

Deux roues de type OSSBERGER ont été testées au barrage de Colliersville sur la rivière Susquehanna (GLOSS et al., 1982) : d'un diamètre de $1 \mathrm{~m}$ et 1,25 $\mathrm{m}$, elles turbinent au maximum respectivement $8,5 \mathrm{~m}^{3} / \mathrm{s}$ et $11,5 \mathrm{~m}^{3} / \mathrm{s}$ sous une chute voisine.de $10 \mathrm{~m}$. Leur vitesse de rotation est de $135 \mathrm{trs} / \mathrm{mn}$ et $104 \mathrm{trs} / \mathrm{mn}$.

Les principaux résultats portent sur les salmonidés (juvéniles de saumon, de truite steelhead, truite arc-en-ciel). Ils montrent que la mortalité est très fortement corrélée à la taille du poisson (fig. 2.6.). Par contre, le régime de fonctionnement des turbines ne semble avoir aucune influence significative. 


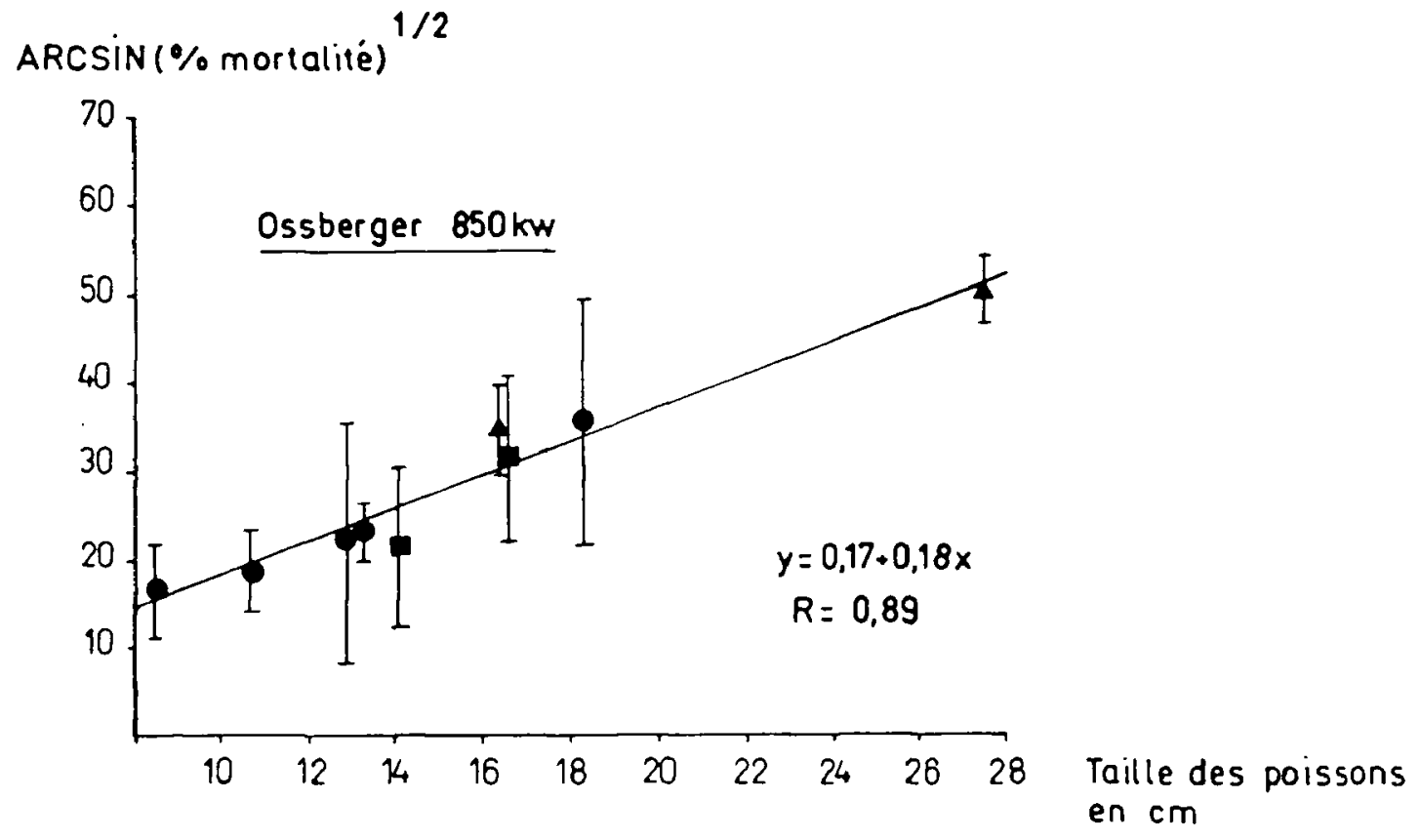

- Saumon atlantique

- Truite de mer

- Truite arc-en-ciel

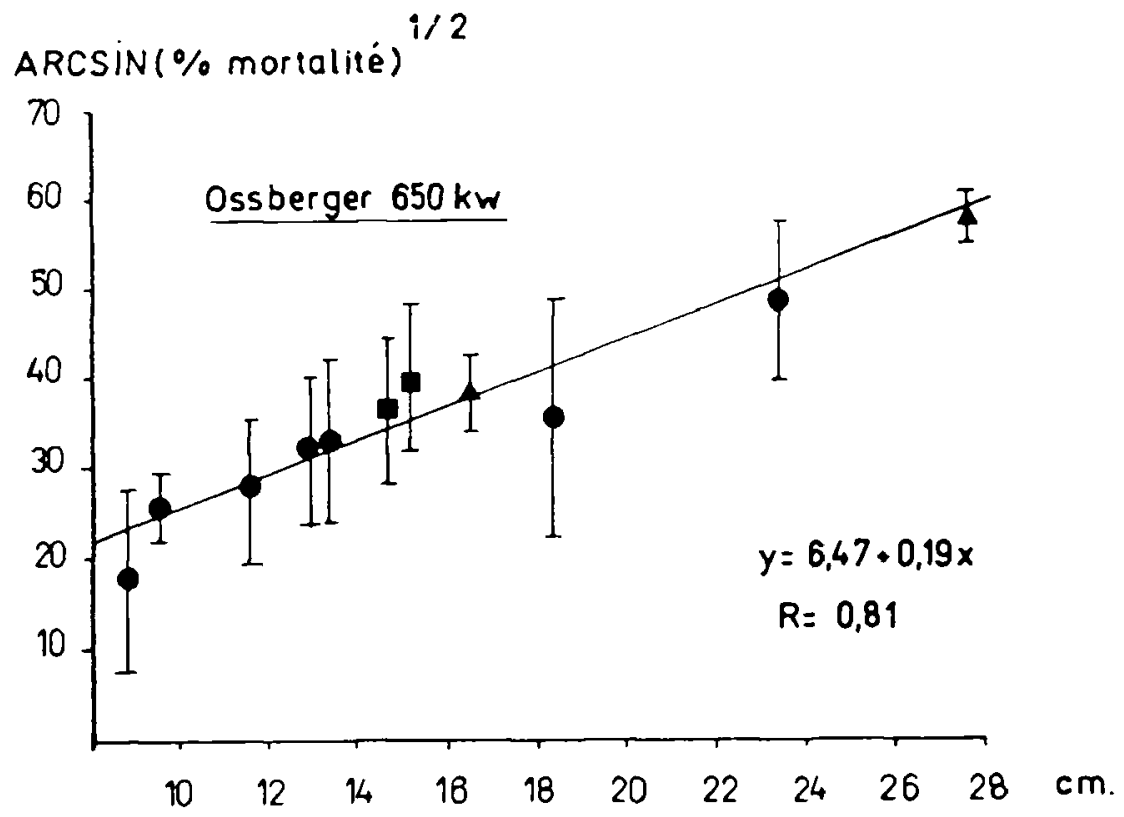

figure 2.6. : Taux de mortalité des poissons en fonction du diamètre de la roue et de la taille des poissons pour deux turbines OSSBERGER (d'après GLOSS et al., 1982).

figure 2.6. : Relationship of fish mortality to turbine diameter and fish length (from GLOSS and al., 1982). 


\subsection{Blessures}

La recherche des causes de mortalité à partir de l'examen des différents types de blessures observées sur le poisson n'a été que rarement effectuée. On citera les travaux menés aux aménagements de Shasta, Cushmam et Big Cliff (U.S. Army Corps of Engineers, 1963 et 1966), de Baker (HAMILTON et ANDREW, 1954), de Seton Creek (ANDREW et GEEN, 1958), de Lequille (Montreal Engineering Company Limited, 1981 et 1982) et de Motala (MONTEN, 1985).

Les blessures ont été classées généralement en trois catégories

- les dommages d'origine mécanique, résultant d'un contact plus ou moins violent du poisson avec un élément fixe ou mobile de la turbine, se manifestant

- extérieurement, par des blessures superficielles comme des abrasions, des contusions, des lacérations, des hémorragies à la base des nageoires, des fractures de la colonne vertébrale, par des blessures plus nettes comme des entailles allant jusqu'au sectionnement du poisson,

- intérieurement par des effets secondaires des chocs, comme des hémorragies dans les parois musculaires, dans divers organes ou dans la cavité générale, un dégonflement de

la vessie gazeuse suite à des perforations par des arêtes,

- les dommages attribués aux gradients de vitesse (cisaillement, "shearing"). Les blessures se caractérisent par des opercules retournés ou arrachés, des arcs branchiaux brisés, des isthmes rompus, voire la séparation totale du corps et de la tête.

- les dommages attribués aux phénomènes de pression, notamment aux dépressions brutales ou à la cavitation. Ils se manifestent:

- extérieurement par des exophtalmies, des hemorragies au niveau des yeux, des yeux crevés ou manquants, certaines parties du corps apparaissant "mâchurées" ("pulping") en l'absence de trace d'impact,

- intérieurement, par des hémorragies d'organes, l'éclatement de la vessie gazeuse sans trace d'impact.

A partir des expérimentations menées à Lequille, RUGGLES et COLLINS (Montreal Engineering Company Limited, 1982) ont effectué une étude statistique sur les différents types de blessure et leur relation éventuelle avec les conditions de fonctionnement de la turbine et la taille du poisson.

L'ouverture des directrices, bien que n'influençant que peu le taux de mortalité global, se fait sentir sur la distribution du type de blessure : à $60 \%$ d'ouverture, la plus grande partie des dommages est composée d'hémorragies internes, de fractures et d'yeux manquants ou crevés. A $75 \%$ d'ouverture, correspondant au rendement maximal, les hémorragies internes sont responsables de la majorité des mortalités. Par contre, à $90 \%$ et $100 \%$ d'ouverture, les poissons sectionnés, le plus souvent au niveau de l'isthme branchial, composent entre $29 \%$ et $49 \%$ des poissons morts recapturés. Le taux de poissons sectionnés ou présentant des fractures augmente significativement avec la taille.

Le pourcentage de mortalité attribuée à la pression seule est estimé à 2,4\% seulement.

Les auteurs attribuent une forte proportion des poissons sectionnés ou présentant des fractures aux effets de cisaillement à l'entrée de la roue dans la mesure où le pourcentage ne semble pas lié à l'espacement entre aubes et directrices alors qu'il semble augmenter avec le débit transitant daris la turbine.

\section{EFFETS DE LA PRESSION ET DE SES VARIATIONS}

La plupart des auteurs ont observé, quelquefois dans de fortes proportions, des dommages qui ne semblaient ni d'origine mécanique, ni causés par des gradients de vitesse et qui ont été attribués aux effets de la pression et de ses variations brutales au passage de la roue. C'est le cas à Baker (HAMILTON et ANDREW, 1954), à Seton Creek (ANDREW et GEEN, 1958), à Tusket Falls (SMITH, 1960 et 1961), à Shasta et Cushman (CRAMER et OLIGHER, 1964); sur cette dernière installation, le pourcentage de poissons morts présentant ce type de dommages atteignait $43 \%$ en 1960.

Les symptômes des dommages attribués aux variations de pression varient cependant avec les auteurs. Parmi les plus fréquemment cités, l'exophtalmie, les yeux crevés ou manquants, les hémorragies internes ou au niveau des yeux, l'éclatement de la vessie natatoire sans trace d'impact.

Un certain nombre d'expérimentations en laboratoire permettent de mieux interpréter les causes des dommages liés aux variations de pression.

Les variations de pressions induisent chez le poisson des mécanismes physiologiques de deux ordres: modification du volume de la vessie natatoire et mouvement des gaz dissous dans l'organisme, en particulier le sang. 


\subsection{La vessie gazeuse}

La plupart des poissons osseux ont un organe appelé vessie natatoire ou gazeuse, diverticule du tube digestif avec lequel il peut su non rester en communication par un conduit, le canal pneumatique. On parle alors respectivement de poisson physostome ou de poisson physocliste. Outre les nombreuses fonctions sensorielles qu'on lui attribue, cet organe, de par la masse gazeuse qu'il contient, joue un rôle hydrostatique; son volume et la pression y régnant satisfont à la loi des gaz parfaits, toute variation relative de la pression s'accompagnant d'une variation relative opposée de son volume $(\mathrm{dv} / \mathrm{V}=-\mathrm{dp} / \mathrm{P})$.

Cependant, lorsque la réduction de pression devient notable $(>10 \mathrm{~cm} \mathrm{Hg}$ ), la dilatation de la vessie reste inférieure à ce que prévoit la loi des gaz parfaits, le péritoine et les tissus environnant la vessie offrant une résistance à l'expansion de celle-ci, qui se trouve alors à une pression supérieure à la pression externe (JONES, 1951).

La régulation du volume de la vessie natatoire par adaptation de sa pression à celle du milieu ambiant permet au poisson de conserver son équilibre hydrostatique.

Chez les espèces physoclistes (perche, sandre), cette régulation s'effectue obligatoirement par échange gazeux avec les vaisseaux sanguins de la paroi de la vessie, le mélange gazeux de la vessie n'ayant d'ailleurs pas forcément la même composition que l'air atmosphérique. Chez les espèces physostomes (salmonidés, clupéidés, cyprinidés) la régulation peut s'effectuer dans certaines circonstances beaucoup plus rapidement par la bouche via le canal pneumatique.

Les poissons tolèrent sans problème de fortes pressions, le seul effet néfaste étant la perte provisoire de l'équilibre hydrostatique causée par la réduction du volume de la vessie gazeuse. En effet, aucun auteur ne mentionne de mortalité consécutive à une augmentation de pression. Les travaux de LAMPERT (1976) ont montré que des espèces physostomes et physoclistes supportaient des mises en pression de 50 atmosphères à une vitesse de $10 \mathrm{~atm} / \mathrm{s}$.

Les risques liés à 'une réduction brutale de la pression sont tout autres : toute réduction de pression se traduit en effet par une augmentation du volume de la vessie natatoire, le poisson ayant donc tendance à remonter à la surface suite à l'augmentation de sa flottabilité. Tant que la réduction de pression n'excède pas $50 \%$ à $60 \%$ de la valeur initiale (dp/Po $<0.5$ à 0.6 ) (JONES, 1952; TSVETKOV, 1971), il ne semble pas y avoir de risques d'éclatement de la vessie gazeuse.

Au-delà de cette limite, toute diminution de pression est dommageable si elle s'effectue à une vitesse supérieure au taux d'élimination ou de réabsorption du gaz excédentaire.

L'élimination du gaz en excès chez les poissons physoclistes ne peut se faire que par réabsorption au travers de la membrane de la vessie. TSVETKOV (1971) évalue ce taux de réabsorption à $10^{-5} \mathrm{~atm} / \mathrm{seconde}$, valeur du même ordre de grandeur que celles observées par d'autres auteurs (JONES, 1952; FEATHERS et KNABLE, 1983)

Par contre, chez les poissons physostomes, le gaz en excès peut être évacue beaucoup plus rapidement par le canal pneumatique - ou par le conduit postérieur chez les clupéidés TSVETKOV (1971) a établi le taux limite de décompression au-delà duquel apparaît le risque de rupture de la membrane de 0,9 à $1 \mathrm{~atm} / \mathrm{seconde}$. Cette valeur-seuil doit cependant être abaissée a $0,1 \mathrm{~atm} / \mathrm{s}$ pour les individus plus jeunes chez qui la membrane est plus fragile et/ou le canal pneumatique peu fonctionnel.

Le risque de rupture de la vessie est donc beaucoup moins grand chez les especes physostomes que chez les espèces physoclistes.

Lors d'une réduction de pression, la vitesse d'évolution de la pression paraît donc le paramètre déterminant sur le taux de survie, beaucoup plus que l'amplitude même de la variatior de pression.

\subsection{La sursaturation en gaz dissous}

Une diminution de la pression ambiante peut se traduire d'autre part par une sursaturation en gaz dissous (2) de l'organisme - particulièrement du sang - du poisson. Le gaz en excès est libéré

(2) Les mouvements des gaz dissous dans le sang et les liquides corporels d'un poisson expose a des variations de pression s'apparentent à ceux que l'on observe pour l'eau. c'est-a-dire qu'ils satisfont à la Loi de Henry: à tempèrature constante, la quantité maximale de gaz qui peut se dissoudre dans un volume de liquide donné est proportionnelle à la pression partielle exercée par ce gaz à la surface du liquide.

Les mécanismes de dissolution et d'élimination d'un gaz par un liquide ou un tissu satisfont à une cinétique du premier ordre:

$$
\mathrm{dC} /(\mathrm{C}-\mathrm{C} 1)=-\mathrm{kdt} \text { ou }(\mathrm{C}-\mathrm{C} 1) /\left(\mathrm{Co}-\mathrm{C} 1-\mathrm{e}^{\mathrm{k}(\mathrm{t})}\right. \text { to }
$$

ou $C$ - concentration au temps $t$

Co - concentration à saturation initiale au temps to à la pression po

C1 - concentration à saturation afférente a pression finale $p 1$.

La vitesse de dégazage initiale est donc proportionnellea la différence des concentrations a saturation. donc à la différence de pression. 
sous forme de bulles pouvant entraîner des embolies ("Gaz Bubble Disease"), voire la rupture des vaisseaux sanguins.

TSVETKOV (1971) note l'apparition de bulles à partir d'une diminution de pression de 1,5 à 2 atmosphères chez la majorité des physostomes testés, alors que les premières mortalités sont constatées à partir d'une réduction de pression de $2 \mathrm{~atm}$, quelle que soit la vitesse de décompression.

Les expérimentations effectuées par BEYER et al., (1976) sur le saumon coho indiquent que les mortalités, encore négligeables pour une réduction de pression de 2 atmosphères, deviennent notables pour des réductions de 3 atmosphères

Les effets dus à l'embolie gazeuse sont beaucoup plus lents à se manifester - plusieurs dizaines de minutes - que ceux consécutifs à la détérioration ou à la rupture de la vessie natatoire, presque instantanés.

Lors d'une réduction de pression, il n'y a risque d'embolie que si le poisson est acclimaté à la pression initiale : le poisson qui, acclimaté à une pression voisine de la pression atmosphérique, est soumis à une brutale augmentation de pression, puis à un retour rapide à la pression atmosphérique ne court aucun risque d'embolie gazeuse; de nombreuses expérimentations le confirment (ROWLEY, 1955; PYPER et al., 1958; MUIR, 1959).

\subsection{La cavitation}

On évoque souvent, lors des essais sur les turbines, des dommages causés par la cavitation. Les expérimentations en laboratoire n'ont jamais apporté de résultats tangibles sur ce point. Les travaux menés par l'U.S. Army Corps of Engineers ont seulement pu établir que des smolts pouvaient supporter des expositions à des très basses pressions, de l'ordre de 0,35 m d'eau, voisines de la tension de vaporisation de l'eau. MUIR (1959), après avoir observé visuellement des poissons exposés à la cavitation, a conclu que la mortalité était consécutive à des chocs : les poissons étaient projetés contre les parois de l'enceinte de confinement lors des variations très brutales de pression accompagnant l'implosion des poches d'air.

\subsection{Conclusions}

Les effets éventuels d'une variation de pression sur le poisson dépendent avant tout de l'adaptation ou non préliminaire du poisson à la pression minimale à laquelle il est soumis.

Le poisson entrant dans une turbine à partir d'une prise d'eau de surface, dont l'organisme est donc adapté à une valeur de la pression voisine de la pression atmosphérique, n'a pas la possibilitè de s'adapter à la pression plus élevée régnant en entrée de roue; le volume de sa vessie gazeuse est initialement réduit en accord avec l'augmentation de pression, puis repasse à son volume initial en sortie de roue. II ne subira aucun dommage.

Cependant, si la pression au passage de la roue subit un abaissement important au-dessous de la pression atmosphérique, supérieur à 5-6 mètres d'eau (correspondant à une réduction de 50-60\% par rapport à la pression initiale à laquelle est adapté le poisson), et ceci à une vitesse supérieure à la vitesse d'adaptation du poisson (c'est-à-dire 0,9-1 atm/s chez les espèces physostomes), il y a un risque d'éclatement de la vessie gazeuse.

Dans le cas d'une prise de fond, si le poisson est adapté à la pression correspondant à la profondeur de celle-ci, le brusque retour à la pression atmosphérique consécutif au passage à travers la turbine pourra entraîner des dommages beaucoup plus considérables.

Sur les juvéniles de salmonidés, migrant dans les couches de surface - a fortiori sur les installations de basses chutes - la pression a peu de chance d'être un facteur notable de mortalité lors du transit par une turbine, sauf si la dépression en sortie de roue est importante.

Le risque lié à une sursaturation en gaz dissous n'existe pas pour les poissons dévalant dans la tranche d'eau de surface, dans la mesure où leur organisme est généralement en équilibre avec une pression voisine de la pression atmosphérique

Chez les poissons adaptés à une profondeur notable, le risque dépend du niveau de saturation en gaz dissous de la tranche d'eau considérée.

Dans tous les cas, les risques de mortalitès dus à une sursaturation en gaz dissous lors du transit à travers une turbine restent faibles tant que la chute ne dépasse pas une vingtaine de mètres d'eau, ce qui correspond à une sursaturation potentielle maximale équivalant à une diminution de pression de 2 atmosphères. 


\section{EFFETS DES GRADIENTS DE VITESSE}

Le cisaillement est souvent cité lors des expérimentations comme l'un des facteurs prımordiaux de mortalite (RUGGLES, 1980 ; TURBAK et al., 1981). Par cisaillement ("shearing") les auteurs sous-entendent gradient transversal de vitesse au sein d'un écoulement; le poisson subirait des dommages lors de son passage au voisinage des parois ou dans des zones à très forte turbulence, ou encore lorsqu'il se trouve à la limite de deux écoulements à vitesses très différentes.

Des expérimentations ont été réalisées en laboratoire pour étudier les dommages subis par des juvéniles de salmonidés arrivant à l'intérieur d'un jet à très forte vitesse dans un bassin d'eau caime (JOHNSON, 1971, 1972), ou bien directement injectés à la périphérie d'un écoulement a tres forte vitesse (GROVES, 1972).

Les essais effectués par JOHNSON montrent que les dommages sont inexistants pour une vitesse de jet de $17 \mathrm{~m} / \mathrm{s}$. Pour une vitesse de $20 \mathrm{~m} / \mathrm{s}$, la mortalité passe à $2,4 \%$. Puis elle augmente rapidement avec la vitesse: elle passe de $7,2 \%$ pour $24 \mathrm{~m} / \mathrm{s}$ à $31 \%$ pour $28 \mathrm{~m} / \mathrm{s}$. Des films a haute vitesse pris par GROVES ont montré que les dommages se produisent quand une partie du corps du poisson rentre en contact avec un écoulement possédant une vitesse très différente de celle du reste du corps, causant une accélération différentielle intense. C'est la région de la tête qui est la plus sujette aux arrachements consécutifs à ces gradients de vitesse. Les blessures peuvent se produire en l'espace de quelques millisecondes. Les dommages les plus fréquemment observés sont des opercules retournés, des yeux rendus prohéminents ou arrachés, des hémorragies internes, voire des décapitations. Les dommages sont généralement plus importants sur les poissons les plus petits.

\section{MODĖLES PRÉDICTIFS DES MORTALITÉS DANS LES TURBINES}

Deux approches différentes ont conduit à l'élaboration de modèles prédictifs:

- formulation de la probabilité de choc du poisson avec une aube ou une pale,

- mise au point d'équations de régression à partir d'une analyse statistique des données expérimentales.

KREITMANN (1931) et VON RABEN (1957) ont proposé des modèles voisins basés sur le rapport du temps nécessaire à un poisson pour couper entièrement la trajectoire d'une aube, au temps mis par cette dernière pour parcourir la distance inter-aubes. La formule proposée par KREITMANN est discutable d'un point de vue conceptuel et ne pourrait être utilisée en toute rigueur que pour des turbines FRANCIS à faible vitesse spécifique. Elle devient inapplicable sur les autres roues FRANCIS ainsi que les turbines KAPLAN. D'où son intérêt limité. L'expérience montre que ces formules surestiment généralement les mortalités alors qu'elles sont supposées ne prendre en compte qu'une partie des dommages, les mortalités d'ordre mécanique : à Lequille, l'application de la formule de VON RABEN prévoit des mortalités supérieures à $100 \%(137 \%)$ alors que les mortalités d'ordre mécanique observèes n'ont jamais dépassé $26 \%$ (Montreal Engineering Limited, 1981).

Le modèle proposé par MONTEN (1985) ne tient pas compte de la vitesse absolue avec laquelle le poisson aborde la roue. Le pourcentage de mortalité est fonction du rapport entre la taille du poisson et l'espace minimal (appelé espace relatif) dont celui-ci dispose pour passer entre deux aubes ou pales, et de la vitesse relative du poisson par rapport aux aubages déterminant la violence des chocs. L'auteur a dú limiter l'application de sa formule au régime de fonctionnement optimal et pour des turbines présentant des vitesses relatives élevées. Le grand mérite de l'approche de MONTEN a été de considérer pour la première fois les conditions de vitesses en entrée de roue, méme si le calcul des triangles des vitesses reste très approximatif.

Dans tous les cas, les modèles précédents ne constituent qu'une évaluation de la part mécanique des dommages et ne prennent pas en compte les autres facteurs de mortalité comme l'effet des gradients de vitesse, des pressions et de leurs variations.

BELL (1967) a cherchè à déterminer des équations de régression montrant l'effet des différentes variables de fonctionnement de la turbine sur la mortalité. Les analyses de 146 observations sur les turbines FRANCIS de Cushman et Shasta ainsi que de 76 observations sur la turbine KAPLAN de Big Cliff montrent que les variables utilisées nexpliquent que 15 à $17 \%$ de la variance. Les variables les plus significatives sur les FRANCIS sont l'ouverture des directrices, le coefficient de Thoma, indicateur du degré de cavitation, ainsi que la longueur du poisson. Sur la turbine KAPLAN, la racine carrée de la chute ainsi que le coefficient de Thoma se sont revelés les plus explicatifs. La limite majeure de cette étude est qu'il est impossible d'extrapoler les résultats à d'autres installations. 


\section{III - EXPÉRIMENTATIONS FRANÇAISES}

\section{INTRODUCTION}

Une série de sept expérimentations a été menée par le CEMAGREF et le Conseil Supérieur de la Pêche, de mars 1984 à juillet 1985, en collaboration, lorsque les sites étaient sa propriété, avec Électricité de France. Ces études ont porté sur les principaux types de turbines - FRANCIS, KAPLAN, HELICE et BANKI-MICHELL - et sur des installations équipant des chutes de $4,5 \mathrm{~m}$ à $120 \mathrm{~m}$. On a repris de façon très synthétique dans la suite du texte les principaux résultats de ces différentes expérimentations qui ont été décrites plus en détail par ailleurs (DARTIGUELONGUE et LARINIER, 1987 ; TRAVADE et al., 1987; DARTIGUELONGUE, 1988).

Ces expérimentations visaient à mettre au point, en premier lieu, un protocole standard permettant d'obtenir très rapidement un ordre de grandeur des mortalités, susceptible d'être utilisé par la suite sur d'autres sites. L'analyse des différents travaux effectués à l'étranger a conduit les auteurs à privilégier le principe de l'introduction du poisson à l'entrée de la roue et de sa récupération immédiate par filtration de la totalité du débit en sortie de turbine. Ces expérimentations devaient d'autre part permettre d'estimer les risques potentiels encourus par les smolts lors de leur migration vers la mer sur des cours d'eau faisant l'objet de plans de restauration des populations de grands salmonidés migrateurs (Nivelle, Gave d'Ossau, Dordogne, Allier). Sur les deux installations testées dans le Puy de Dôme, il s'agissait d'évaluer le préjudice potentiel que pouvaient représenter deux turbines de petites dimensions et tournant très vite, pour les populations d'alevins de truite.

Les expérimentations sont présentées selon un ordre chronologique, ce qui permet de suivre l'évolution de la technique et du matériel. Les caractéristiques des turbines sont présentées dans le tableau III. 1

\section{MiCRocentrale de SAINT-PÉE-SUR-NIVELLE (PYRÉNÉES-ATLANTIQUES)}

Cette microcentrale, installée sur la rivière Nivelle, équipe une chute de $4,3 \mathrm{~m}$ de deux turbines de type HELICE de $0,60 \mathrm{~m}$ de diamètre tournant à $460 \mathrm{trs} / \mathrm{mn}$ pour un débit nominal de $2,5 \mathrm{~m}^{3} / \mathrm{s}$. les essais se sont déroulés en mars 1984 .

\subsection{Méthode et protocole}

Les poissons sont introduits dans la chambre d'eau de l'une des turbines, en aval des grilles de protection et le plus près possible de l'entrée de la roue, grâce à un tuyau souple de $10 \mathrm{~cm}$ de diamètre et de $5 \mathrm{~m}$ de longueur comportant un entonnoir à son extrémité supérieure.

Lors des premiers lâchers-tests, de nombreux poissons se sont échappésvers l'amont dans le canal d'amenée, ce qui était rendu possible par la faiblesse des vitesses dans la chambre d'eau. Pour limiter le taux d'échappement, il a été procédé à une légère anesthésie des poissons avant leur introduction.

La récupération du poisson s'effectue grâce à un filet de forme pyramidale fixé à la sortie des diffuseurs, dont il a les dimensions en entrée $(2,3 \mathrm{~m} \times 1,85 \mathrm{~m})$. Ce filet en mailles de $14 \mathrm{~mm}$ a une longueur de $10 \mathrm{~m}$; à son extrémité aval est fixé un vivier flottant destiné à récupérer les poissons et à les stocker pendant toute la durée du test.

Deux cent quarante smolts de saumon atlantique d’élevage, de 17 à $26 \mathrm{~cm}$ de longueur (moyenne de $20 \mathrm{~cm}$ ), ont été utilisés. Trois classes de tailles $(\langle 18 \mathrm{~cm}, 18$ à $21 \mathrm{~cm}$ et $\rangle 21 \mathrm{~cm}$ ) ont étè distinguées. Un lot témoin, destiné à évaluer l'impact du matériel et de la méthode, a été introduit directement dans le filet par l'intermédiaire du tube souple; ce lot n'ayant subi aucun dommage, on en a déduit la neutralité des méthodes d'injection et de recapture sur les mortalités observées.

Tous les lots-tests, ainsi que le lot témoin ont été gardés trois semaines en observation.

\subsection{Résultats}

Les taux de récupération se sont révélés très variables du fait des faibles vitesses, tant au point d'introduction qu'à la sortie des aspirateurs où les poissons pouvaient se maintenir sans difficulté

L'emploi de l'anesthésiant a tout de même permis de réduire les pertes, faisant passer le taux de récupération de 61,5 à $91,3 \%$.

Une analyse de variance a montré que l'utilisation de l'anesthésiant n'avait aucune influence significative sur le taux de mortalité $\left(F_{1,2}=4,87, p>0,05\right)$.

Compte-tenu de la variabilité dans les taux de récupération, le calcul de la mortalité a consisté à rapporter le nombre de poissons récupérés morts, soit au nombre de poissons lâchés (limite supérieure de la mortalité), soit au nombre de poissons récupérés (limite inférieure de la mortalité).

La mortalité immédiate est en moyenne égale à $21 \%$ et atteint $35 \%$ après 3 semaines de délai ces pourcentages passent respectivement à $28 \%$ et $46 \%$ si l'on rapporte le nombre de poissons morts au nombre de poissons récupérés.

Aucune liaison significative entre la taille des poissons et le type de blessure ou le pourcentage de mortalité n'a èté mise en évidence. 
Sur une cinquantaine de poissons morts examinés, $8 \%$ ne présentaient aucune blessure apparente; la majorité des blessures externes étaient d'origine mécanique : $30 \%$ des poissons morts présentaient un corps sectionné, $20,4 \%$ avaient la colonne vertébrale brisée, $36,7 \%$ montraient des traces d'impacts graves ou des dépressions importantes sur les flancs, 4, $1 \%$ des poissons avaient la tête arrachée non pas suite à un choc, mais vraisemblablement par l'effet des gradients transversaux ou longitudinaux des vitesses à la traversée de la roue.

L'autopsie de 34 poissons morts entiers a révélé 32,4\% d'hémorragies de la cavité générale, $53 \%$ d'hémorragies d'organes spécifiques (notamment le rein, 26,5\% d'hémorragies des parois musculaires (hématomes consécutifs à des chocs). Outre ces hémorragies, on a observé, dans $30 \%$ des cas, un dégonflement de la vessie gazeuse que l'on a pu attribuer soit à un choc mécanique ou à une perforation par une arête, soit éventuellement à la variation brutale de pression subie lors du passage à travers la roue.

\section{CENTRALE HYDROÉLECTRIQUE DE POUTĖS-MONISTROL (HAUTE-LOIRE)}

L'aménagement hydroélectrique de Poutès-Monistrol sur le Haut-Allier, est constitué d'un barrage d'une vingtaine de mètres de hauteur, d'une dérivation de plus de $9 \mathrm{~km}$ de longueur constituée d'une galerie puis d'une conduite forcée amenant l'eau à deux turbines Francis. La chute nette est de $61 \mathrm{~m}$, la puissance installée de 16,9 MW. Les turbines Francis ont un diamètre de $1,15 \mathrm{~m}$ en entrée, leur débit nominal est voisin de $15 \mathrm{~m} / \mathrm{s}$. Les essais se sont déroulés en mai 1984.

\subsection{Méthode et protocole}

La méthode est identique à celle utilisée précédemment : introduction des poissons dans la turbine puis filtration de la totalité de son débit.

L'introduction des poissons a lieu au barrage, au niveau de la prise d'eau située à une dizaine de mètres au-dessous de la cote normale du plan d'eau de la retenue. Le temps de transit des poissons dans les ouvrages de dérivation jusqu'à l'usine peut varier de $15 \mathrm{mn}$ à $45 \mathrm{mn}$ suivant le débit turbiné.

La récupération des poissons se fait grâce à un filet d'une vingtaine de mètres de long, fixé sur un cadre métallique aux dimensions de la sortie des diffuseurs $(3,7 \mathrm{~m} \times 2,05 \mathrm{~m})$ à laquelle il est fixé. Les mailles sont d'une dimension plus importante dans les premiers mètres du filet ( $22 \mathrm{~mm}$ au lieu de $14 \mathrm{~mm}$ plus à l'aval), afin de réduire les efforts de traction sur la nappe aux forts débits. Une nasse, disposée à l'extrémité aval du filet, permet de récupérer les poissons lors des tests.

Tableau III.1 : Caractéristiques des turbines testées.

Table III.1 : Characteristics of the tested turbines.

\begin{tabular}{|c|c|c|c|c|c|c|c|c|}
\hline $\begin{array}{c}\text { Inslailations } \\
\text { Hydrodlectriques }\end{array}$ & $\begin{array}{l}\text { Type de } \\
\text { Turbine }\end{array}$ & $\begin{array}{l}\text { Hauteur de } \\
\text { Chule } \\
\text { (m) }\end{array}$ & $\begin{array}{l}\text { Debit } \\
\left(\mathrm{m}^{\mathrm{3}} / \mathrm{s}\right)\end{array}$ & $\begin{array}{c}\text { Puissance } \\
\text { Nominale } \\
\text { (MMI) }\end{array}$ & $\begin{array}{l}\text { Vitesse de } \\
\text { Rotation } \\
\text { Trstmm }\end{array}$ & \multicolumn{2}{|c|}{$\begin{array}{c}\text { Diamére de roue } \\
\text { en }\end{array}$} & $\begin{array}{c}\text { Nombre de } \\
\text { Pales } \\
\text { au } \\
\text { D'aubes }\end{array}$ \\
\hline MICRO CENTRALES & & & & & & & & \\
\hline $\begin{array}{l}\text { ST PEE SUR NIVELLE } \\
\text { (Nivelle) }\end{array}$ & Helice & 4.3 & 2.5 & - & 460 & & & 4 \\
\hline $\begin{array}{l}\text { LAILHACAR } \\
\text { (Gave d"Ossau) }\end{array}$ & Kaplan & 5.0 & 19.8 & 0.920 & 165 & & & 4 \\
\hline $\begin{array}{c}\text { COUzON } \\
\text { (Le Couzon) }\end{array}$ & Francis & 97.0 & 0.35 & 0.260 & 1500 & 0.37 & 0.26 & 9 \\
\hline $\begin{array}{c}\text { FAYE } \\
\text { (La Faye })\end{array}$ & Ossbergar & 120.0 & 0.8 & 0820 & 750 & & & - \\
\hline USINES E.D.F & & & & & & & & \\
\hline $\begin{array}{l}\text { POUTES } \\
\text { (Allier) }\end{array}$ & Franas & 61.5 & 14.0 & 8.4 & 428 & 1.15 & 1.45 & 15 \\
\hline $\begin{array}{l}\text { MAUZAC } \\
\text { (Dordogne) }\end{array}$ & Francis & 5.0 & 60.0 & 2.8 & 55 & 4.45 & 4.8 & 14 \\
\hline $\begin{array}{l}\text { TUILIERE } \\
\text { (Dordogne) }\end{array}$ & Kaplan & 115 & 52.4 & 47 & 166.7 & & & 5 \\
\hline
\end{tabular}


Trois ouvertures de la turbine, correspondant à des débits voisins de $8 \mathrm{~m}^{3} / \mathrm{s}, 10,5 \mathrm{~m}^{3 /} / \mathrm{s}$ et $14 \mathrm{~m}^{3} / \mathrm{s}$, ont èté testées; on a procédé au lâcher d'un lot témoin directement dans le filet pou!r prendre en compte l'impact du matériel et de la méthode ainsi que d'un lot de poissons préalablement tués pour tenir compte d'une éventuelle différence de récupération entre poissons morts et poissons vivants. Près de 600 truitelles arc-en-ciel et fario d'élevage, de taille moyenne $16,2 \mathrm{~cm}, 0 \mathrm{nt}$ été utilisées.

\subsection{Résultats (Tableau III.2)}

Aucune mortalité n'a été observée sur le lot témoin introduit directement dans le filet.

Le taux de récupération des poissons morts injectés au niveau de la prise d'eau a atteint $100 \%$, alors que le taux moyen de récupération sur les lots tests a été de $74 \%$.

Le calcul de la mortalité a consisté à rapporter le nombre de poissons morts récupérés au nombre de poissons lâchés sachant que la récupération des poissons morts est totale.

Les taux moyens de mortalité sont respectivement de $48 \%, 35 \%$, et $41 \%$ pour les débits de $14 \mathrm{~m}^{3} / \mathrm{s}, 10,5 \mathrm{~m}^{3} / \mathrm{s}$ et $8 \mathrm{~m}^{3} / \mathrm{s}$. II n'y a pas de différence significative entre ces pourcentages de mortalité

Tableau III.2 : Taux de mortalitè, nombre de poissons lãchés et pourcentages de recapture, turbine de Poutès.

Table III.2 : Rate of mortality, numbers of fish released and recapture percentage, Poutes turbine.

\begin{tabular}{|c|c|c|c|c|}
\hline & $\begin{array}{c}\text { Cónditions } \\
\text { des } \\
\text { Tests }\end{array}$ & $\begin{array}{c}\text { Nombre } \\
\text { de } \\
\text { Poissons lactios }\end{array}$ & $\begin{array}{c}\text { Taux } \\
\text { de } \\
\text { Recapture }\end{array}$ & $\begin{array}{l}\text { Talx de } \\
\text { Malalité }\end{array}$ \\
\hline \multirow{3}{*}{ 惫岕 } & $8.0 \mathrm{~m}^{3} / \mathrm{s}$ & 100 & 61.0 & 41.0 \\
\hline & $10.5 \mathrm{~m}^{3} \mathrm{~s}$ & 200 & 67.5 & 35.5 \\
\hline & $14.0 \mathrm{~m}^{3} \mathrm{~s}$ & 100 & 93.0 & 48.0 \\
\hline \multirow{2}{*}{$\stackrel{5}{6}$} & $\begin{array}{l}\text { POISSONS } \\
\text { MORTS }\end{array}$ & 70 & 100.0 & \multirow{2}{*}{41.3} \\
\hline & TEMOINS & 100 & 100.0 & \\
\hline
\end{tabular}

\subsection{L'étude des blessures}

Cent trente quatre poissons morts ont été examinés :

- à débit nominal, près de $48 \%$ des poissons présentent des blessures d'origine mécanique (corps sectionnés, entaillés, traces d'impact...); $25 \%$ des blessures peuvent être attribuées au cisaillement et $12,5 \%$ à la pression.

- à ouverture réduite, les poissons morts présentent surtout des blessures que l'on a attribué aux effets de pression ( $22 \%$ et $42 \%)$ ou aux effets des gradients de vitesses $(48,9 \%$ et $31,7 \%$ des poissons présentent des arcs branchiaux brisés, des opercules retournés, ou des têtes arrachées) ; les mortalités d'origine mécanique ne représentent que 16 et $20 \%$ des mortalités.

- sur l'ensemble des essais, près de $17 \%$ des poissons morts ne présentent aucun signe externe ou interne de dommage. 


\section{MICROCENTRALE DE LAILHACAR (PYRÉNÉES-ATLANTIQUES)}

Cette microcentrale, située sur le Gave d'Ossau, équipe une chute de $5 \mathrm{~m}$ avec une turbine KAPLAN de $2,10 \mathrm{~m}$ de diamètre et de débit nominal voisin de $19 \mathrm{~m}^{3} / \mathrm{s}$. Les essais ont été effectués en octobre 1984.

\subsection{Méthode et protocole}

La méthode est identique à celle utilisée lors des expérimentations précédentes. Les poissons légèrement anesthésiés, sont introduits le plus près possible de la turbine, au moyen d'un tuyau souple. Leur récupération se fait par filtration du débit de la turbine. Le cadre métallique $(6.7 \mathrm{~m}$ par $3,5 \mathrm{~m}$ ), auquel est fixé le filet, coulisse dans les rainures de batardage ménagées à la sortie de l'aspirateur. Le filet de forme pyramidale (fig. 3.1.) a une longueur de $17 \mathrm{~m}$, la surface totale de la nappe étant voisine de $170 \mathrm{~m}^{2}$. La dimension des mailles $(20 \mathrm{~mm}, 16 \mathrm{~mm}$ et $11 \mathrm{~mm})$ décroît de l'entrée vers la nasse $(0,50 \mathrm{~m} \times 0,50 \mathrm{~m} \times 1 \mathrm{~m})$. La manipulation de l'ensemble cadre-filet nécessite des moyens de levage appropriés (pelle hydraulique).

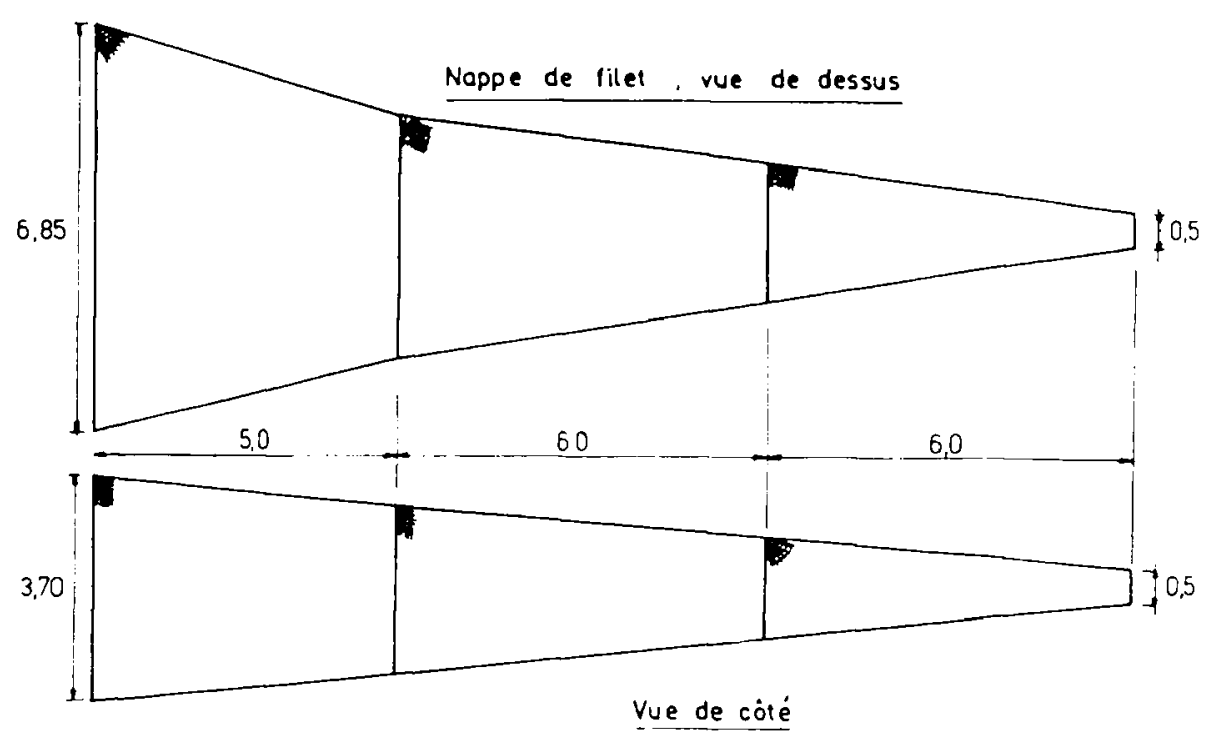

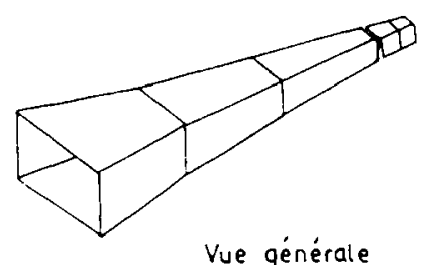

Vue générale

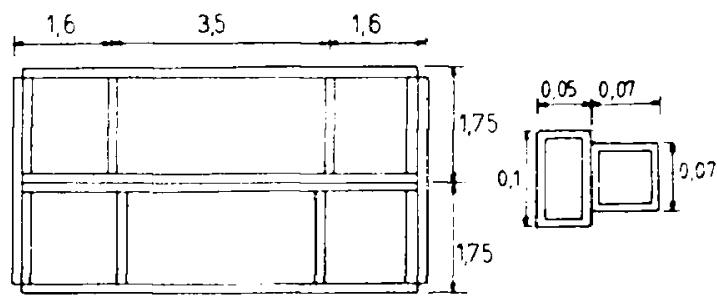

Cadre métallique supportant le filet

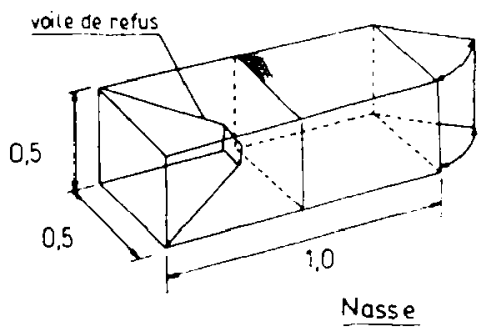

Toutes les mesures soni en mères

figure 3.1. : Schèma du système de récupération, expérimentation à la centrale de Laïlhacar. figure 3.1. : : Schematic diagram of the recovery net, Laïlhacar powerhouse tests. 
Les taux de mortalité ont été évalués pour trois ouvertures de directrices (débits voisins de $7 \mathrm{~m} / \mathrm{s}, 13 \mathrm{~m}^{3} / \mathrm{s}$ et $19 \mathrm{~m} / \mathrm{s}$ ). Les poissons utilisés sont des juvéniles de truite fario répartis en deux classes de taille (de moyennes respectives de $14,8 \mathrm{~cm}$ et $18,5 \mathrm{~cm}$ ) constituant pour moitié chacun des trois lots-tests de 100 poissons (soit au total 435 poissons en comptant le lot témoin et les lots de poissons morts). Une fois récupérés, les poissons sont transportés puis gardés en observation pendant une semaine en pisciculture.

\subsection{Résultats (Tableau III.3)}

Le taux de mortalité observé sur le lot témoin (2\%) montre la quasi-neutralité du matériel.

Le taux.moyen de récupération sur les lots-tests s'est avéré relativement faible (68\%), compte tenu que la totalité du débit est filtrée.

Le taux important de récupération des poissons morts (87\%) laisse supposer que la majorité des poissons non recupérés sont vivants.

La mortalité brute a étè corrigée de façon à prendre en compte la mortalité due au matériel et la différence de taux de recapture entre poissons vivants et poissons morts. Le taux de mortalité corrigé est donné par l'expression :

$$
\mathrm{X}=(1 / \mathrm{Nb} \cdot \operatorname{Trm}) \cdot(\mathrm{Rm}-\mathrm{Tmf}(\mathrm{Rv} /(1-\operatorname{Tmf})))
$$

où :

Nb est le nombre de poissons lâchés

Trm, le taux de recapture des poissons morts

$\mathrm{Rm}$, le nombre de poissons retirés morts du filet

Tmf, le taux de mortalité sur le lot témoin

$R v$, le nombre de poissons retirés vivants du filet

La mortalité immédiate varie suivant les essais de $4 \%$ à $9,5 \%$, et la mortalité après une semaine de délai varie de $4 \%$ à $11,5 \%$.

Les analyses de variance n'ont pas montré de liaison significative entre taille du poisson et mortalité, et entre le régime de fonctionnement et la mortalité, bien que cette dernière soit plus faible à pleine ouverture (4\%) qu'à ouverture partielle $(9,5 \%$ et $11,5 \%)$.

Le faible nombre de poissons morts (25 au total) ne permet pas d'aller très loin dans l'analyse des types de blessure. Les dommages les plus fréquents $(48 \%)$ sont ceux liés aux gradients de vitesse - cisaillement ou élongation - (têtes arrachées, opercules retournés ou arcs branchiaux brisés), ou à des chocs mécaniques (32\% des poissons morts présentent des traces d'impacts ou des entailles).

Tableau III.3 : Taux de mortalité, nombre de poissons lâchés et pourcentages de recapture, turbine de Lailhacar.

Table III.3 : Rate of mortality, numbers of fish released and recapture percentage, Laïlhacar turbine.

\begin{tabular}{|c|c|c|c|c|c|c|c|}
\hline & \multirow{3}{*}{$\begin{array}{c}\text { Conditons } \\
\text { des } \\
\text { Tests }\end{array}$} & \multirow{3}{*}{$\begin{array}{c}\text { Nombre } \\
\text { de } \\
\text { Lachès }\end{array}$} & \multirow{3}{*}{$\begin{array}{c}T a\lrcorner x \\
z e \\
\text { Recaptute }\end{array}$} & \multicolumn{4}{|c|}{ Taux de mortalite } \\
\hline & & & & \multicolumn{2}{|c|}{ Immedia! } & \multicolumn{2}{|c|}{ Apres 7 jours } \\
\hline & & & & Brut & Corrigè & Brut & Corrige \\
\hline \multirow{3}{*}{ 象总 } & $7 \mathrm{~m}^{3} \mathrm{~s}$ & 100 & 290 & 90 & 9.5 & 11.0 & 11.5 \\
\hline & $13 \mathrm{~m}^{\prime} \mathrm{is}$ & 95 & 579 & 9.5 & 85 & 10.5 & 95 \\
\hline & $19 \mathrm{~m}^{3 / \mathrm{s}}$ & 100 & $6: 0$ & 40 & 40 & 40 & 40 \\
\hline \multirow{2}{*}{ ๘ } & POISSONS & & & \multicolumn{4}{|c|}{ MOYENAN } \\
\hline & TEMOINS & 105 & $\because c$ & & & & \\
\hline
\end{tabular}




\section{MICROCENTRALES SUR LE LE COUZON ET LA FAYE (PUY-DE-DOME)}

Ces deux microcentrales, installées sur des ruisseaux, le Couzon et la Faye, équipent respectivement des chutes de $97 \mathrm{~m}$ et $120 \mathrm{~m}$ de deux turbines FRANCIS de $37 \mathrm{~cm}$ de diamètre (debit nominal de $0,350 \mathrm{~m}^{3} / \mathrm{s}$ ) tournant à $1.500 \mathrm{trs} / \mathrm{mn}$ et d'une turbine OSSBERGER de $60 \mathrm{~cm}$ de diamètre (débit maximal de $0,800 \mathrm{~m}^{3} / \mathrm{s}$ ) tournant à $750 \mathrm{trs} / \mathrm{mn}$. Dans les deux cas, l'eau est amenée de la prise d'eau à l'usine par une conduite forcée de 2 à $3 \mathrm{~km}$ de long. Les essais se sont déroulés en juin 1985.

\subsection{Méthode et protocole}

Les poissons sont introduits au niveau de la prise d'eau grâce à un tuyau glissé dans le reniflard. Le temps de transit moyen des poissons a été évalué au préalable par l'injection de fluorescéine. Pour limiter ce temps de transit et garantir des vitesses suffisantes dans la conduite, les turbines étaient mises à leur ouverture maximale pendant quelques minutes immédiatement aprés l'introduction des poissons.

La récupération des poissons s'effectuait grâce à des filets coniques disposés dans les canaux de fuite en béton restituant les eaux turbinées au ruisseau. La faible longueur de ces canaux a conduit à l'installation de filets très courts (environ $3 \mathrm{~m}$ ), ayant des angles d'ouverture importants (supérieurs à $50^{\circ}$ ), peu favorables à un bon guidage des poissons vers la nasse.

Sur le Couzon, les essais ont été effectués pour trois ouvertures de la turbine FRANCIS (1/2, $3 / 4$ et pleine charge) sur trois classes de taille de poissons $(3 \mathrm{~cm}, 10 \mathrm{~cm}$ et $16,7 \mathrm{~cm})$. Sur la Faye, trois régimes de fonctionnement ont été testés, définis par la largeur de la roue utilisée et le pourcentage du débit maximal ( $2 / 3$ et $80 \%, 3 / 3$ et $68 \%, 3 / 3$ et $40 \%)$. Trois classes de tailles ont été injectées $(3 \mathrm{~cm}$, $5,6 \mathrm{~cm}$ et $10,2 \mathrm{~cm})$.

A ces lâchers tests, ont étè rajoutés des lâchers de lots témoins et de lots de poissons morts, ce qui porte à 600 le nombre de truitelles utilisées.

\subsection{Résultats (Tableau III.4.)}

Les taux de mortalité sur les lots témoins ont été très élévés, notamment sur la plus petite classe de tailles pour laquelle ils ont atteint $100 \%$. Pour les deux autres classes de tailles, la mortalité n'est significative que sur la Faye, probablement du fait des conditions climatiques rencontrées lors des expérimentations (température de l'eau élevée et temps orageux).

Tableau III.4 : Taux de mortalité, nombre de poissons lâchés et pourcentages de recapture, turbine du Couzon.

Table III.4 : Rate of mortality, numbers of fish released and recapture percentage, Couzon turbine.

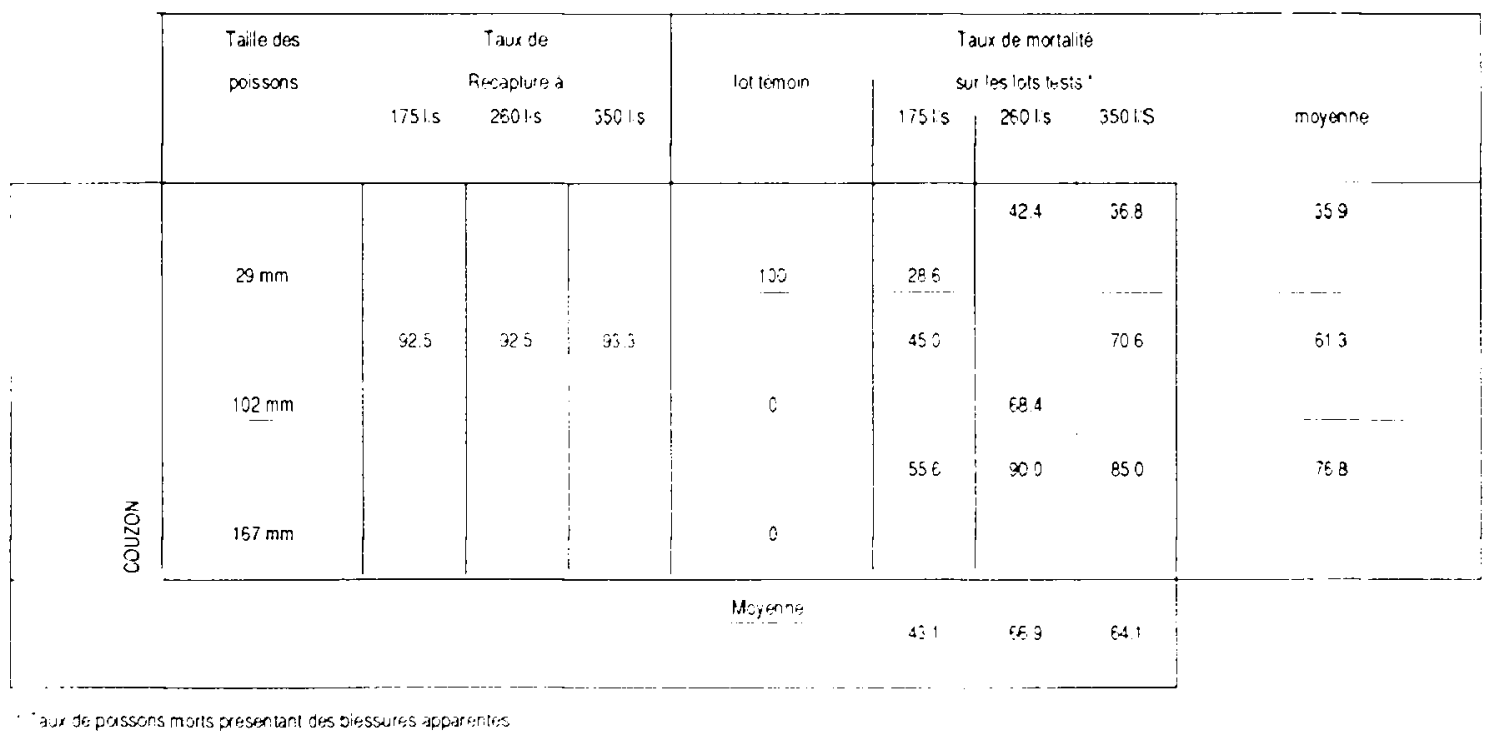


Tableau III.4 : Taux de mortalité, nombre de poissons lâchés et pourcentages de recapture, turbine de la Faye.

Table III.4 : Rate of mortality, numbers of fish released and recapture percentage, Faye turbine.

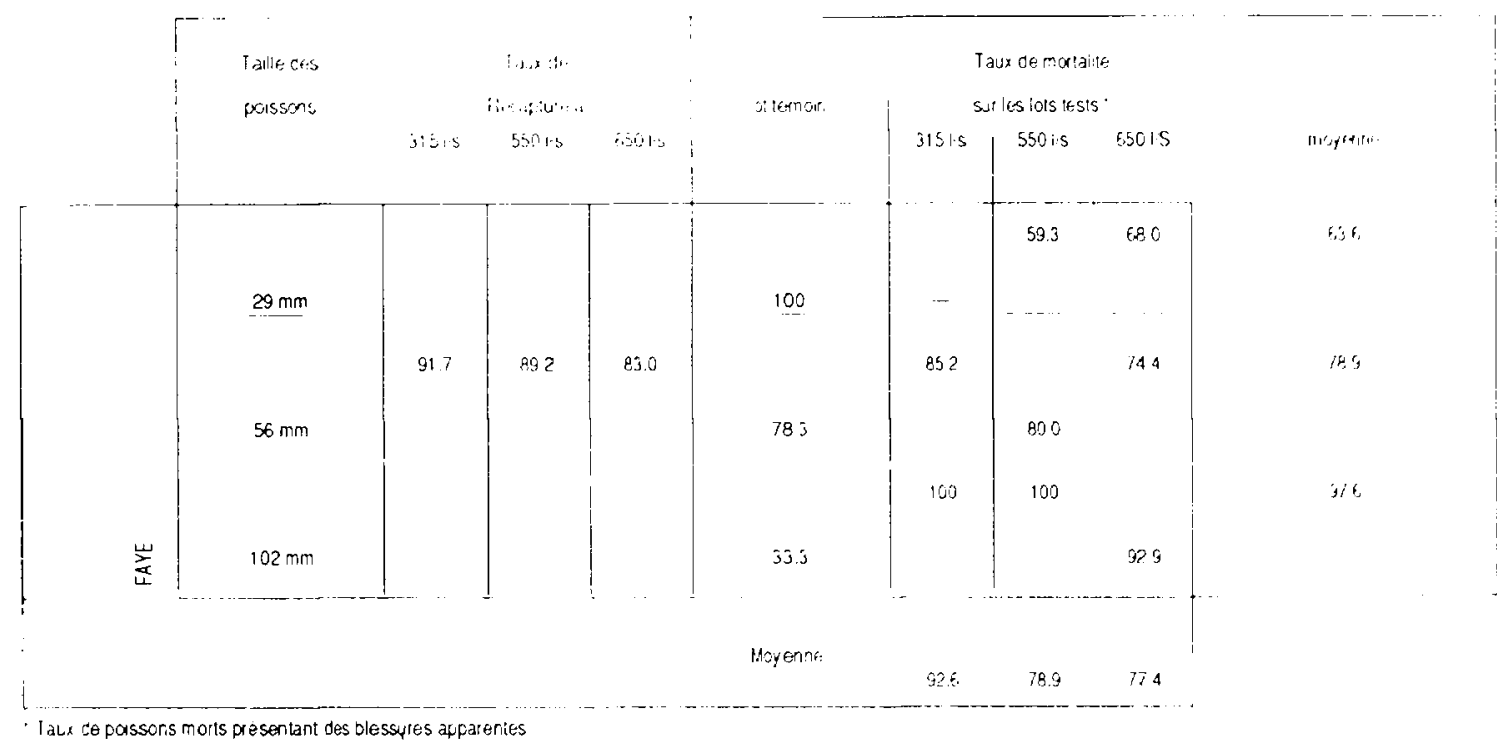

Les taux de recupération des poissons morts sont equivalents à ceux des lots tests, soit pròs de $93 \%$ sur le Couzon et en moyenne $88 \%$ sur la Faye; la présence sur les deux sites d'une fosse de dissipation sous la turbine créant des zones de recirculation où les poissons demeurent fiérgés explique les taux de poissons non récupérés.

On a alors défini un taux de mortalité "minimale" correspondant aux pourcentages de poissons récupérés morts et présentant des dommages apparents, sachant que les poissons témoins morts ne présentaient aucun signe de blessure.

Sur le Couzon, la mortalité "minimale" par blessure varie de $43 \%$ à près de $67 \%$. Ces taux de blessures sont significativement liés à la taille du poisson ( $\left.F_{2.4}=12,12, p<0,05\right)$ et au régime de fonctionnement de la turbine $\left(F_{2.4}=30,4, p<0,01\right)$.

Sur la Faye, la mortalité "minimale" a varié de $77,4 \%$ à près de $93 \%$. Les pourcentages de blessures augmentent significativement avec la taille di pcissun ( $\left.F_{L .5}=21,29, p<0,01\right)$.

Ces forts pourcentages de blessures d'origine mécanique étaient prévisibles compte-tenu des vitesses de rotation élevées $(1.500$ et $750 \mathrm{trs} / \mathrm{mn})$ et des faibles espacements entre aubes $(2,5 \mathrm{~cm}$ sur le Couzon et $3 \mathrm{~cm}$ sur la Faye). Il est même étonnant qu'ils n'aient pas été p!us élevés.

\section{CENTRALE hYDROÉLECTRIQUE DE MAUZAC (DORDOGNE)}

La centrale nydroélectrique E.D.F. de Mauzac sur la Dordogne (chute brute voisine de $5 \mathrm{~m}$, puissance installée 18,2 MW) est équipée d'une turbine KAPLAN et de cinq turbines FRANCIS identiques. La turbine FRANCIS testée (à axe vertical), d'un diamètre de 4,45 m en entrée et tournant à $55 \mathrm{trs} / \mathrm{mn}$ a un débit nominal de $60 \mathrm{~m}^{3} / \mathrm{s}$. Les essais se sont déroulés en juillet 1985.

\subsection{Méthode et protocole}

Le but de cette expérimentation, outre l'appréciation des dommages potentiels sur ce site. visait à adapter une technique mise au point sur des installations de tailles modestes à un ouvrage installé sur un cours d'eau plus important. De ce fait, la méthodologie est identique à celle utilisée antérieurement: introduction des poissons le plus près possible de la turbine et récupération immédiatement à l'aval par filtration de la totalité du débit.

Le cadre métallique nécessaire à la fixation du filet a été construit aux dimensions de la rainure de batardage $(11 \mathrm{~m} \times 7 \mathrm{~m})$. La surface du filet a été calculée de manière à ce que le rapport du débit à filtrer sur la surface du filet n'excède pas $25 \mathrm{~cm} / \mathrm{s}$, la section d'entrée étant fixée par les dimensions des aspirateurs. Les poissons sont récupérés dans une nasse fixèe à un radeau.

Compte-tenu de la dimension de la turbine (près de $4,5 \mathrm{~m}$ de diamètre) et de l'expérience acquise, le facteur taille du poisson n'a pas semblé déterminant: les lots n'ont comporté qu'une seule classe de tailles, de moyenne $19.5 \mathrm{~cm}$.

Les tests ont porté sur les régimes de fonctionnement de la turbine les plus courants: pleine charge et mi-charge. En prenant en compte les lots témoins et les lots de poissons morts. près de 400 truitelles fario d'élevage ont été injectèes dans la turbine. 


\subsection{Résultats (T ableau III.5)}

Le taux de récupération sur les poissons morts a été de $100 \%$, ce qui permet de faire l'hypothèse que les poissons non récupèrés sont des poissons vivants.

Les taux de récupération sur les lots tests ont été de $93 \%$ et $96 \%$.

Le taux de mortalité sur le lot témoin a été de $3 \%$

La mortalité immèdiate a été de $8 \%$ lors des deux essais et de $5,3 \%$ et $5,4 \%$ après correction Ces valeurs ne montrent donc pas de différence significative selon le régime de fonctionnement.

Le nombre de poissons morts était trop faible pour permettre une analyse des blessures observées.

Tableau III.5 : Taux de mortalitè, nombre de poissons lâchés et pourcentages de recapture, turbine de Mauzac.

Table III.5 : Rate of mortality, numbers of fish released and recapture percentage, Mauzac turbine.

\begin{tabular}{|c|c|c|c|c|c|}
\hline & $\begin{array}{c}\text { Conditions } \\
\text { des } \\
\text { Tesis }\end{array}$ & $\begin{array}{c}\text { Nombie } \\
\text { de } \\
\text { Poissons laches }\end{array}$ & $\begin{array}{c}\text { Taux } \\
\text { de } \\
\text { Recapture }\end{array}$ & Brul & Corrigè \\
\hline \multirow{2}{*}{ 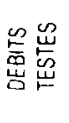 } & $55 \mathrm{~m}^{3} \mathrm{~s}$ & 100 & 96 & 80 & 5.3 \\
\hline & $30 \mathrm{~m}^{3} \mathrm{is}$ & 100 & $\$ \hat{3}$ & 80 & 5.4 \\
\hline \multirow{2}{*}{ 号 } & TEMOIN & 100 & 90 & 3.0 & \\
\hline & $\begin{array}{l}\text { POISSONS } \\
\text { MORTS }\end{array}$ & 100 & 100 & & \\
\hline
\end{tabular}

\section{CENTRALE hydRoÉleCtRIQUE DE TUILIĖRES (DORDOGNE)}

La centrale hydroélectrique E.D.F. de Tuilières sur la Dordogne (chute brute de $11.5 \mathrm{~m}$, puissance installèe de $37,6 \mathrm{MW}$ ) est équipée de huit roues KAPLAN turbinant au maximum un débit voisin de $400 \mathrm{~m}^{3} / \mathrm{s}$. La turbine testée est une KAPLAN à axe vertical de $2,9 \mathrm{~m}$ de diamètre, tournant à $167 \mathrm{trs} / \mathrm{mn}$, d'un débit nominal de $52 \mathrm{~m}^{3} / \mathrm{s}$.

\subsection{Méthode et protocole}

La méthodologie, en particulier le mode de recapture, est identique à celle utilisée à la centrale de Mauzac.

Le matériel de récupération est adapté aux dimensions de l'ouvrage. Ainsi, le cadre métallique fait $7 \mathrm{~m} \times 3.5 \mathrm{~m}$ et le filet $26 \mathrm{~m}$ de longueur.

Quatre régimes de fonctionnement ont été testés (1/4,1/2,3/4 de la charge et pleine charge).

Quatre lots de 100 poissons chacun, un lot témoin et un lot de poissons morts ont été utilisès: pour limiter le temps de manipulation, quelques minutes après lintroduction du dernier poisson, une "chasse" à plein régime de la turbine permet d'accélérer le transit des poissons jusqu'à la nasse.

Six cents truitelles fario d'élevage, de taille moyenne de $19,5 \mathrm{~cm}$, ont été introduites soit dans la turbine, soit directement dans le filet pour le lot témoin.

Les essais ont eu lieu en juillet 1985, à la suite des essais effectués à Mauzac.

\subsection{Résultats (Tableau III.6)}

Le taux de mortalité sur le lot témoin a étè voisin de $30 \%$ : cela est surtout dù aux coriditions climatiques et notamment à une temperature de l'eau qui a atteint près de $25^{\circ} \mathrm{C}$ lors des essais.

Le taux de récupération des poissons morts est de $100 \%$ et les taux de récupération sur les lots-tests sont supérieurs à $96 \%$. 
La valeur trop élevée de mortalité sur les lots témoins ne nous a pas permis d'utiliser le mode de correction dans le calcul de la mortalité adopté lors des expérimentations précédentes. 11 est apparu plus réaliste de ne prendre en compte, pour l'estimation d'une mortalité "minimale", que le nombre de poissons présentant des blessures visibles extérieurement ou intérieurement.

La mortalité "minimale" varie de $8,3 \%$ à $17 \%$, selon le régime de fonctionnement. Une analyse statistique ne révèle pas de liaison significative avec le régime de fonctionnement.

Soixante quatre poissons morts ont été examinés, sans que l'on puisse mettre en évidence une liaison entre le régime de fonctionnement et le type de blessures.

Tableau III.6 : Taux de mortalité, nombre de poissons lâchés et pourcentages de recapture, turbine de Tuilières.

Table Ill.6 : Rate of mortality, numbers of lish released and recapture percentage, Tuilières turbine.

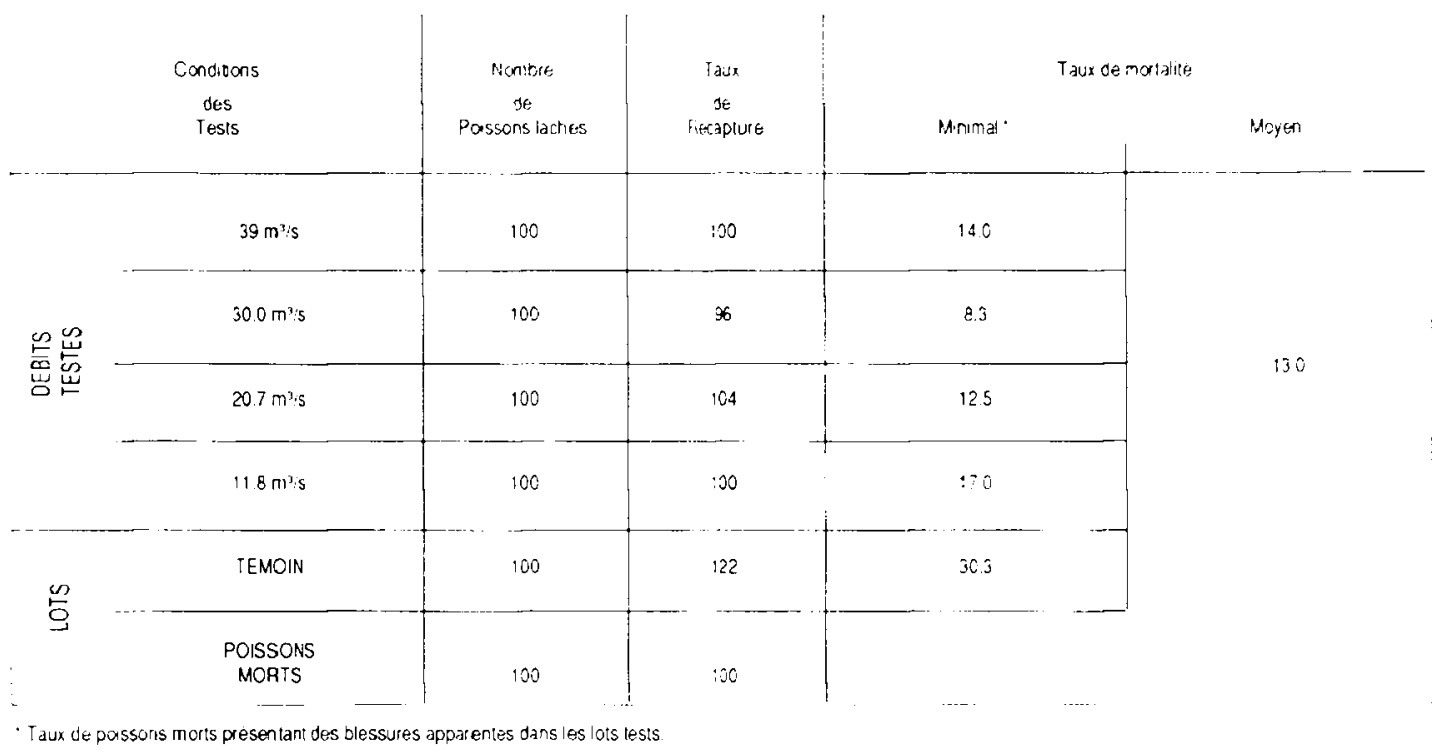

\section{CONCLUSIONS}

\subsection{Sur la méthode}

Cette série d'expérimentations a permis de tester une méthodologie basée sur la filtration de la totalité du débit de la turbine, qui permet d'obtenir relativement rapidement un ordre de grandeur des dommages potentiels sur une installation donnée, en injectant un nombre raisonnable de poissons.

Les moyens à mettre en œuvre, tant en matériel qu'en personnel, deviennent cependant très lourds lorsque les débits à filtrer atteignent plusieurs dizaines de $\mathrm{m}^{3} / \mathrm{s}$. II semble qu'une centaine de $\mathrm{m}^{3} / \mathrm{s}$ soit la limite à partir de laquelle il faille se tourner soit vers la technique plus sophistiquée du radiopistage, soit vers une méthode d'échantillonnage plus souple. mais qui en contrepartie nécessitera l'introduction de lots-tests beaucoup plus conséquents (plusieurs milliers de poissons).

La technique utilisée est grandement facilitée par la présence, à la sortie des aspirateurs, d'une rainure de batardage où glissera le cadre auquel est accroché le filet. Un moyen de levage approprié pour manipuler cadre et filet devient indispensable dès que le débit dépasse quelques $\mathrm{m}^{3 / \mathrm{s}}$, compte tenu du poids du cadre imposé par les efforts importants de traction transmis par le filet.

La température est un paramètre du milieu primordial qui peut affecter à la fois les lots-tests et les lots témoins: sur trois des expérimentations effectuées, la mortalité importante observée sur les lots de contrôle résulte vraisemblablement des conditions climatiques critiques (température de l'eau élevée) rendant toute manipulation des poissons délicate.

La période d'expérimentation doit être choisie de manière à éviter les périodes de chute des feuilles, car le colmatage rapide du filet qui en découle augmente les risques de dommage des poissons par plaquage, de déchirure du filet (cas à Lailhacar), voire de rupture du cadre. 
II semble difficile de travailler avec des individus de petites tailles - en deçà de $4 \mathrm{~cm}$-comme on a pu en faire l'expérience sur la Faye ou le Couzon, et comme le rapportent d'autres auteurs (U.S. Army Corps of Engineers, 1963; BELL et BRUYA, 1981; MONTEN, 1985).

En plus des lâchers de lots de poissons nécessaires à l'étude des facteurs de mortalité (taille des poissons, régime de fonctionnement de la turbine...), il convient de procéder systématiquement à des lâchers:

- de lots témoins, afin d'évaluer l'impact de la méthode (transport, manipulations, stabulation) ainsi que du matériel d'introduction et de récupération; dès que les pourcentages de mortalité sur les lots témoins deviennent importants, l'appréciation des dommages attribuables au transit dans la roue devient beaucoup plus délicate.

- de lots de poissons préalablement tués, afin de prendre en compte une éventuelle différence entre les taux de recapture des poissons vivants et des poissons morts.

L'emploi de l'anesthésiant - en dose légère - s'est avéré nécessaire lorsque les vitesses de l'eau au point d'introduction étaient trop faibles (cas à St-Pée-sur-Nivelle). La passivité des poissons dans les premiers mètres évite le problème de l'échappement vers l'amont. Cette anesthésie ne semble pas affecter le taux de mortalité.

Tableau III.7 : Caractéristiques des filets de recapture utilisés lors des expérimentations sur le transit des poissons à travers les turbines.

Table III.7 : Characteristics of recovery nets used in tests on fish passage through hydraulic turbines.

\begin{tabular}{|c|c|c|c|c|c|c|c|c|c|}
\hline Instaliation & Debits & & mension du filet & & Surface & Dimension & Riaport & Angle as & Dimension \\
\hline ST PEE SUR NIVELLE & 2.5 & 10 & $1.85 \times 2.3$ & $0.2 \times 0.2$ & 45 & $\begin{array}{l}14 \\
10\end{array}$ & .05 & 13 & Viner \\
\hline LAILHACAR & 19 & 17 & $3,5 \times 6,7$ & $0.5 \times 0.5$ & 170 & 20.16 el 11 & 0.12 & 21 & $\begin{array}{l}1 \times 5 \times .5 \\
\cos 6 \mathrm{~mm}\end{array}$ \\
\hline EOUZON & 0.711 & 2.3 & $2.48 \times 0.312$ & $0.2 \times 0.2$ & $4.5 ; 3\}$ & 5 & 0.15 & 52 & $5 \times 2 \times 2$ \\
\hline FAYE & $0.8(4)$ & 2.3 & $32,0 \hat{0} 2$ & $0.2 \times 02$ & $5.7 i 3 j$ & 5 & 0.15 & est & $5 \times 2 \times 2$ \\
\hline POUTES & 14 & 15 & $3.7 \times 2.05$ & $0.5 \times 0.5$ & 85 & $22 e 114$ & 0.16 & $: 2$ & $: 5 \times 5 \times 5$ \\
\hline TUILIERE & 39 & 26 & $7 \times 3,5$ & $0.5 \times 0.5$ & 275 & 20 e1 15 & 0,14 & 14 & $: 5: 5 \times .5$ \\
\hline MAUZAC & $5 \$$ & 15 & $11 \times 7$ & $0,5 \times 0.5$ & 250 & $22 \mathrm{et}: 14$ & 0.22 & $j 8$ & $.5 \times 5 \times 5$ \\
\hline SHASTA & BS & 27 & $4.2 \times 3.9$ & 0.9309 & 270 & $19 e: 14$ & 0.50 & 7 & nise \\
\hline EULIERSVILE & 115 & 135 & $45 \times 3$ & 0.32 & 105 & 13,12 elE.25 & $01:$ & 19 & $n, n \in A$ \\
\hline NECNAR & 30 & $\because$ & $15 \times 60$ & & 65 & 80,60 er 40 & 305 & $i:$ & - \\
\hline LEOUiLLE & 11.3 & 15 & $3: 2$ & c.is & 81 & is & 0.14 & 12 & NuEr \\
\hline LeOiJille & $11 . j$ & 20 & $3.1 \times 2$ & 0.15 & 109 & 13 & 0.10 & 9 & $\ln \theta$ \\
\hline
\end{tabular}

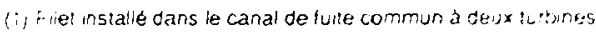

2. Section d'entree moulliee (aporox/matii)

(3) Surface de filet moullee (approximatit)

1.: File: iristalle dans le canal de fulte 
II est nécessaire d'étudier le trajet des poissons jusqu'à la turbine, afin de s'assurer de l'absence de zones d'eaux mortes propices au piégeage des poissons (cas à Tuilières) qui peuvent induire des mélanges entre différents lots. Dans le doute, on devra recourir à un marquage des poissons, par exemple un lot sur deux (ablation de l'adipeuse, d'une partie de la caudale...).

La nappe de filet doit être dimensionnée de façon qu'elle soit gonflée par l'écoulement en évitant cependant que les vitesses aux mailles ne deviennent trop fortes. Un rapport débit maximum/surface de la nappe de filet (exprimés respectivement en $\mathrm{m}^{3} / \mathrm{s} \mathrm{et} \mathrm{m}^{2}$ ) voisin de $0,15 \mathrm{~m} / \mathrm{s}$ semble une valeur acceptable. On a porté sur la figure 3.2. et le tableau III.7 les relations débit maximum filtré-surface de filet afférentes à différentes expérimentations effectuées en France ou à l'étranger.

Pour assurer un guidage correct du poisson vers la nasse, l'angle au sommet de la pyramide formée par le filet doit rester limité: $30^{\circ}$ semble une bonne valeur, $40^{\circ}$ étant un maximum.

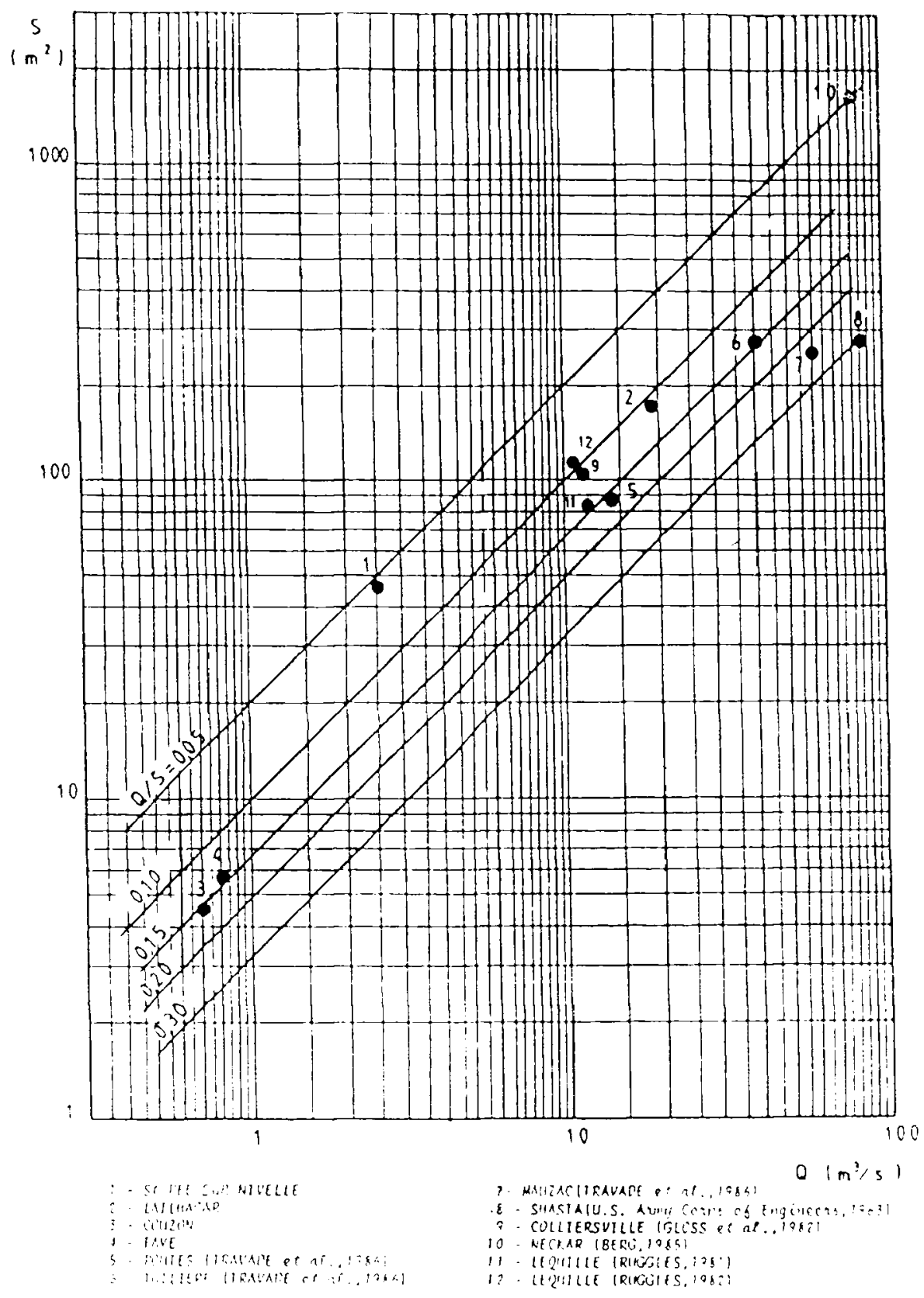

figure 3.2. : : Relațion entre le débit filtré et la surface du filet de récupération utilisé. figure 3.2. : : Relationship of recovery fyke net area to filtrated discharge. 
Les deux critères sur l'angle d'ouverture et le rapport débit/surface de la nappe, ainsi que les caractéristiques de la sortie de l'aspirateur, permettent de donner les principales caractéristiques du filet pour un site donné.

Dès que le débit à filtrer devient conséquent (supérieur à $10 \mathrm{~m}^{3} / \mathrm{s}$ ), un filet avec des mailles de différentes tailles, décroissantes de l'entrée vers la nasse, est souhaitable:

- des mailles de gros diamètre en entrée du filet évitent une trop grande tension sur le filê dans une zone où la turbulence est généralement encore très forte.

- des mailles de plus petits diamètres, disposées en bout de filet assurent le guidage et la rétention correcte des poissons.

Pour les tests à ouvertures partielles de la turbine, il s'avère souvent nécessaire d'effectuer une "chasse" de quelques minutes après le passage du lot-test dans la turbine de façon a récupérêr plus facilement et rapidement les poissons dans la nasse.

\subsection{Sur les dommages}

Bien que le but initial de la série d'expérimentations décrite brièvement ci-avant résidait a l'origine dans la mise au point d'un protocole expérimental, il a été possible de dégager certaıns résultats sur les dommages subis par les poissons.

Le taux de survie des poissons apparaît comme une fonction croissante du diamètre de la turbine pour une taille de poisson donnée: les dommages se sont révélés beaucoup plus importants sur les turbines de petites tailles (Couzon, St-Pée-sur-Nivelle) que sur les grandes (Mauzac, Lailhacar, Tuilières) bien qu'il soit hasardeux de comparer des turbines fonctionnant sous des chutes différentes.

Dans la majorité des cas, la mortalité apparaît consécutive soit à des blessures d'origine mécanique, soit à des blessures que l'on peut attribuer aux gradients de vitesses: l'influence de la pression et/ou de ses variations n'a pas semblé trés évidente sur les installations testées.

L'observation des dommages lors de ces expérimentations nous a permis de mieux comprendre les descriptions faites dans la littérature par les différents auteurs et de prendre conscience des difficultés à identifier l'origine des dommages, du fait de la similitude de certaines blessures.

A cet égard, les blessures que l'on a attribué aux gradients de vitesses, comme les têtes arrachées ou les opercules retournés, ont toutes les apparences de blessures consécutives à des chocs mécaniques. Cependant, leur observation précise montre que les abords de la blessure sont toujours nets, sans marque, alors que dans le cas d'un choc mécanique, des hématomes sont visibles à la périphérie, et qu'il est possible, dans le cas particulier d'une décapitation, de déceler des traces d'impact.

Lorsqu'il est mentionné, ce type de blessure est presque toujours attribué aux effets de cisaillement ("shear effect"), c'est-à-dire aux gradients de vitesse transversaux. Nos observations nous inclinent à penser qu'il peut s'agir aussi bien d'une conséquence de l'étirement du poisson pris dans un gradient longitudinal de vitesse. Ce peut être le cas par exemple, lorsque le poisson subit une brusque accélération: en entrée de la turbine de l'usine de Poutes, un poisson passe en quelques centièmes de seconde, d'une vitesse voisine de $5 \mathrm{~m} / \mathrm{s}$ à une vitesse supérieure à $24 \mathrm{~m} / \mathrm{s}$. 


\section{IV - ANALYSE DES DONNÉES ET MODĖLES PRÉDICTIFS}

\section{INTRODUCTION}

Les observations effectuées lors des expérimentations sur la nature de certaines biessures et leur origine possible, comme les gradients tongitudinaux ou transversaux de vitesse, ont amené les auteurs à tenter de prendre en considération, dans l'analyse des données, les conditions de vitesse et de pression régnant dans la roue au moment du passage du poisson. Une telle démarche avait été esquissée sans grand succès par BELL et BRUYA (1981) sur le site de Foster et par MONTEN (1985) en Suède; cet auteur a d'ailleurs été le premier à développer l'idée d'une vitesse critique d'impact associèe à une probabilité de contact du poisson avec une pale ou une aube. La restriction des causes de mortalité aux seuls chocs mécaniques et le fait de ne prendre en compte qu'un nombre: réduit d'expérimentations ne lui ont cependant pas permis d'aller très loin dans la prédiction des taux de mortalité.

On a donc cherché à déterminer pour un certain nombre d'expérimentations effectués en France et à l'ètranger, les vitesses absolues et relatives en entrèe et en sortie de rout. Cotts: reconstitution n'a été évidemment possible que pour les expérimentations pour lesquelles la géométrie de la roue, ainsi que ses conditions de fonctionnement lors des essais $1 \%$ ouverture, débit. chute, etc...) étaient connues avec suffisamment de précision. Les vitesses ont été calculées a mi-aubes pour les turbines FRANCIS et à mi-pales pour les turbines KAPLAN, ce choix étant guidé par le fait que les trajectoires des poissons sont a priori inconnues et que dans ces conditions. ce sont ces triangles de vitesses qui caractérisent le mieux les conditions d'écoulement dans la roue Les méthodes de calcul des triangles des vitesses ont été reportées dans l'annexe $B$.

La prise en compte d'une variable indicatrice des dépressions subies par le poisson au passage de la turbine - le coefficient de Thoma par exemple - n'a pas été possible car sa détermination nécessitait la connaissance des cotes relatives du plan d'eau aval et du plan de sortie de la roue pour tous les essais considérés. Or ces données n'étaient disponibles que sur une minorité d'expérimentations.

Sur les installations pour lesquelles un nombre très important d'essais avaient été effectués (Cushman, Shasta, Lequille), n'ont été conservés, dans le souci de ne pas donner trop d'importancie aux turbines en question, que les essais les plus indiscutables, c'est-à-dire ayant des mortalites s:Ir les lots témoins nulles ou négligeables.

85 essais sur les juvéniles de salmonidés ont été retenus pour les turbines FRANCIS, essáls afférents aux expérimentations effectuées sur les 15 sites suivants: le Couzon (DARTIGUELONGUE et LARINIER, 1987), Poutes et Mauzac (TRAVADE et al., 1987), Stornorrfors, Upper Hemsjö et Motala (MONTEN, 1985), Lower Eiwha et Glines Canyon (SCHOENEMAN et JUNGE, 1954), CUshman (CRAMER et OLIGHER, 1964), Shasta (U.S. Army Corps of Engineers, 1963), Puntledge (C.;i). Dept. of Fisheries, 1958), Seton (ANDREW et GEEN, 1958), Baker (HAMILTON et ANDREW, 1954), Ruskin (HAMILTON et ANDREW, 1953), Lequille (Montreal Engineering Ltd. 1981, 1982).

58 essais sur les juveniles de salmonidés ont èté retenus pour les turbines KAPLAN ou les groupes BULBES, essais afférents aux expérimentations effectuées sur les 14 sites suivants Lailhacar (DARTIGUELONGUE et LARINIER, 1987). Tuitières (TRAVADE et al., 1987), Angabäck, Langgöl et Motala (MONTEN, 1985), Rock Island (OLSON et KACZINSKI, 1980), Essex (KNIGHT et KUZMESKUS, 1982), McNary (SCHOENEMAN etal., 1961), Foster (BELL et BRUYA, 1981), Big Cliff (OLIGHER et DONALDSON, 1966; U.S. Army Corps of Engineers, 1979), Lower Monumental (LONG et al. 1975). Holyoke (KNAPP et al., 1982), Tusket's Falls (SMITH, 1960 et 1961), Tobique Narrows (MC EACHERN, 1960). A ces essais il convient d'ajouter 22 tests effectués en Suède en 1960 et 1961 sur le transit des anguilles à travers les turbines KAPLAN des 8 usines de Kvarnaholm, Broby, Nöbbelöv, Emsfors, Karlnäs, Gustavsberg, Langgöl et Angabäck (MONTEN, 1985).

Une analyse détaillée de ces données a été effectuée par l'un des auteurs (DARTIGUELONGUE. 1988) par différentes méthodes : analyse factorielle des correspondances. analvse discriminante, régressions linèaires. Nous ne reprendrons dans la suite que l'étude par régression dans la mesure où l'on était intéressé avant tout à dégager des expressions susceptibles de prédire sur une turbine de caractéristiques données l'ordre de grandeur des mortalités sur des poissons d'une taille connue. Les formules explicitées dans la suite peuvent différer légèrement de celles proposées dans le travail original: certaines données relativement récentes, utilisées à l'origine pour tester les formules prédictives, ont été intégrées dans de nouveaux traitements dans le souci de prendre en compte un maximum de sites différents. 


\section{LES VARIABLES}

Les régressions n'ont pas èté effectuées directement sur les pourcentages de mortalitè mais sur la variable transformée ARCSIN (\% mortalité) ${ }^{0.5}$, classiquement utilisée lorsqu'on travaille sur des pourcentages (TOMASSONE et al., 1983), et qui sera notée AMO. Cette transformation, ayant tendance à normaliser les variances, possède la caractéristique de dilater les échelles dans les domaines des très faibles et des très fortes mortalités et à écraser le domaine des mortalités moyennes.

On a distingué quatre types de variables explicatives dans les régressions:

- La taille du poisson ( $T L$ ), exprimée en mètre.

- Les caractéristiques générales de la turbine, qui sont très facilement accessibles pour tout aménagement

- le diamètre de la roue en entrée $\mathrm{D} 1 \mathrm{~m}$, à mi-aube pour les FRANCIS et à mi-pale pour les KAPLAN (voir fig. 1.4. et 1.5.), exprimé en mètres.

- le nombre de pales ou d'aubes (NAP).

- le débit $(Q)$, exprimé en $\mathrm{m}^{3} / \mathrm{s}$,

- la chute (Haut), exprimée en mètres,

- la vitesse de rotation ( $N$ ), exprimée en $\mathrm{trs} / \mathrm{mn}$,

- la vitesse spécifique (NS), exprimée en trs/mn,

- la vitesse périphérique à mi-pale ou mi-aube $\mathrm{U} 1$, exprimée en $\mathrm{m} / \mathrm{s}$,

- la puissance (Puis), exprimée en MW.

On notera par (QN), (HautN), (NSN), (PuisN), les valeurs nominales du débit ( $Q$ ), de la hauteur de chute nette (Haut), de la vitesse spécifique (NS), et de la puissance (Puis). Ces valeurs caractérisent une turbine donnée.

- des variables correspondant à des grandeurs plus physiques, en particulier les valeurs approximatives en entrée et en sortie de roue des vitesses relatives ( $W_{1}$ et $\left.W_{2}\right)$ et absolues ( $V_{1}$ et $\left.V_{2}\right)$, les composantes normales et périphériques de ces dernières $(V 1 n, V 1 u$, et $V 2 n, V 2 u)$, le rendement hydraulique de la turbine $m^{h}$ ainsi que les angles $\alpha$ et $\beta$ (Alf1, Bet1, Alf2, Bet2) en entrée et en sortie caractérisant les triangles des vitesses; les valeurs de toutes ces variables sont liées à la fois aux caractéristiques nominales de la turbine et à ses conditions de fonctionnement. On rappelle que les triangles des vitesses ont été déterminés à mi-aubes ou à mi-pales. Toutes les vitesses seront exprimées en $\mathrm{m} / \mathrm{s}$ et les angles en degrés.

- des variables composées "conceptuelles", pouvant associer de manière plus ou moins subjective certaines variables décrivant la géométrie de la roue, les champs de vitesse dans cette roue ainsi que la taille du poisson et qui seraient indicatrices de certains types particuliers de dommages (DARTIGUELONGUE, 1988). Nous ne mentionnerons dans la suite que les plus significatives et celles issues de l'analyse bibliographique. Parmi elles seront considérées les expressions proposées par VON RABEN (1957) et par MONTEN (1985), que l'on notera respectivement (RAB) et (Mont)

L'expression (RAB), formulation de la probabilité de choc, est le rapport du temps (T1) nécessaire à un poisson pour couper entièrement la trajectoire de l'arête d'entrée (bord d'attaque) d'une aube au temps (T2) mis par cette aube pour parcourir la distance inter-aube.

$$
T 1=T L / V 1 \quad T 2=(T . D 1 m / N A P) /(T . D 1 m . N / 60)
$$

D'ou :

$$
R A B=N \cdot T L \cdot N A P /(60, V 1)
$$

L'espacement relatif (Mont) défini par MONTEN est le rapport entre la taille du poisson et deux fois l'espace minimal dont il dispose pour passer entre deux aubes ou deux pales:

$$
\text { Mont }=\mathrm{TL} /(2 \text {. esp. SIN(Bet1)) }
$$

(esp) est la distance inter-aubes ou inter-pales calculée le long du cercle d'entrée :

$$
\text { esp }=T . D 1 m / N A P
$$

(Bet1), l'angle d'incidence de l'écoulement en entrée, est peu différent de l'angle d'inclinaison des pales sur les turbines KAPLAN. Sur les turbines FRANCIS, cet angle n'est voisin de celui des aubes que dans les conditions optimales de fonctionnement de la roue.

Comme autres variables testees, citons (Mont1), (TL/esp), (CC) et (Tou)

(Mont1) dérive de (Mont) en remplaçant l'angle d'incidence de l'écoulement par l'angle d'inclinaison des aubes ou des pales en entrée (Betn). (Betn) est constant dans le cas d'une turbine FRANCIS. (Mont1) et (Mont) sont égaux ou tout au moins très voisins dans le cas des turbines KAPLAN. 
La variable (TL/esp), rapport de la taille du poisson et de la distance inter-aubes ou intf; - palt::, possède par rapport aux variables précédentes l'avantage de la simplicité de calcul.

(CC) est une vitesse de choc définie en thèrie des turbomachines (VIVIER, 1966) commr: Iit quantité dont il faudrait augmenter ou diminuer la vitesse périphérique U1 pour rétablir le trianglr: des vitesses normal, c'est-à-dire sans chocs:

$$
\mathrm{CC}=\operatorname{IV} 1 \mathrm{~m} .(\operatorname{cotg}(\text { Bet } 1-\operatorname{cotg}(\text { Betn)}))
$$

Les pourcentages de mortalités consécutifs à des chocs devant être a priori fonction, norn seulement de la probabilité de choc, mais aussi de sa violence, certaines variables ont èté introduites, qui combinent l'effet de la taille du poisson et des vitesses en entrée de roue. Parmi tout 5 s less variables testées, citons (Tou), variable voisine de (RAB) et (Mont), intégrant la vitesse relativi; d: l'écoulement en entrée de roue.

$$
\text { Tou }=\text { - W1. (N.TL/esp) })^{1 / 2}
$$

\section{PRÉDICTION DES MORTALITÉS DANS LES TURBINES FRANCIS}

\subsection{A partir des caractéristiques nominales.}

Les paramètres les plus corrèlés à la mortalité sont la hauteur de chute nominale (HautN) (coefficient de corrélation $R=0.66$ ), la vitesse périphérique à mi-aube $U 1(R=0.62)$, le nombre d'aubes (NAP) $(R=-0.61)$, la vitesse spécifique ( $\mathrm{R}=-0.51$ ) ainsi que la vitesse de rotation $N$ $(R=0.45)$. La mortalité semble peu liée au seul paramètre biologique, la taille du poisson $(R=0.20)$.

La régression simple

$$
\text { AMO }=16.22+0.226 \text { HautN }
$$

explique $43 \%$ de la variance.

L'équation de régression à quatre variables suivante explique $70 \%$ de la variance de la mortalité $(R=0.85)$ :

$$
\mathrm{AMO}=6.54+0.218 \mathrm{HautN}+118 \mathrm{TL}-3.88 \mathrm{D} 1 \mathrm{~m}+0.0078 \mathrm{~N}
$$

Une fois ces quatre variables prises en compte, l'influence des autres facteurs n'est plus significative.

Cette expression permet d'obtenir un taux moyen de mortalité pour une taille de poissor, donnée indépendamment des conditions de fonctionnement de la turbine considé rée, en particulie de son ouverture.

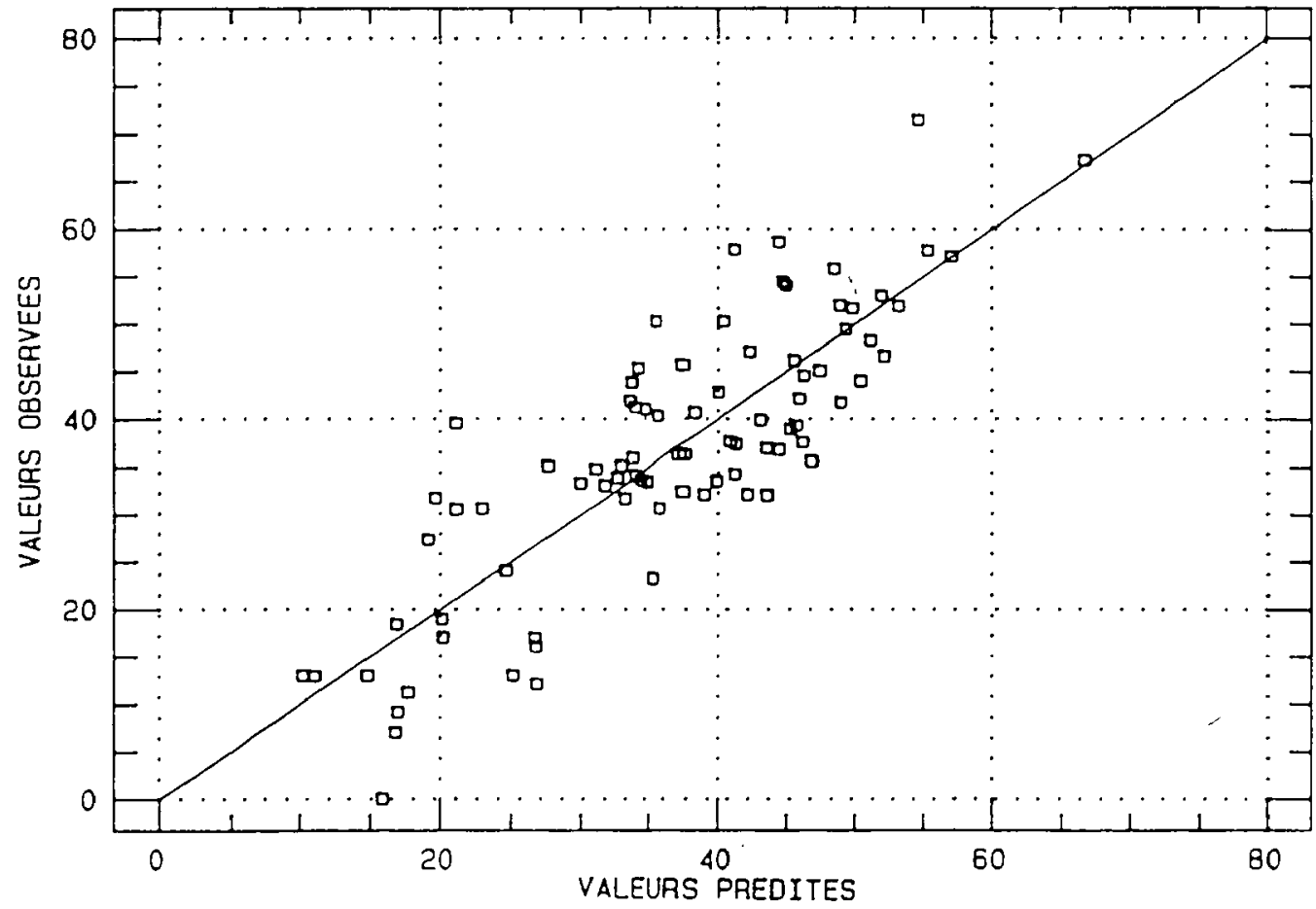

figure 4.1. : Valeurs prédites des taux de mortalité transformés (variable AMO) en fonction des valeurs observées. Régression linéaire sur les turbines FRANCIS.

figure 4.1. : : Relationship of predicted transformed mortalities (AMO) with observed mortalities. Linear regression on FRANCIS turbines. 


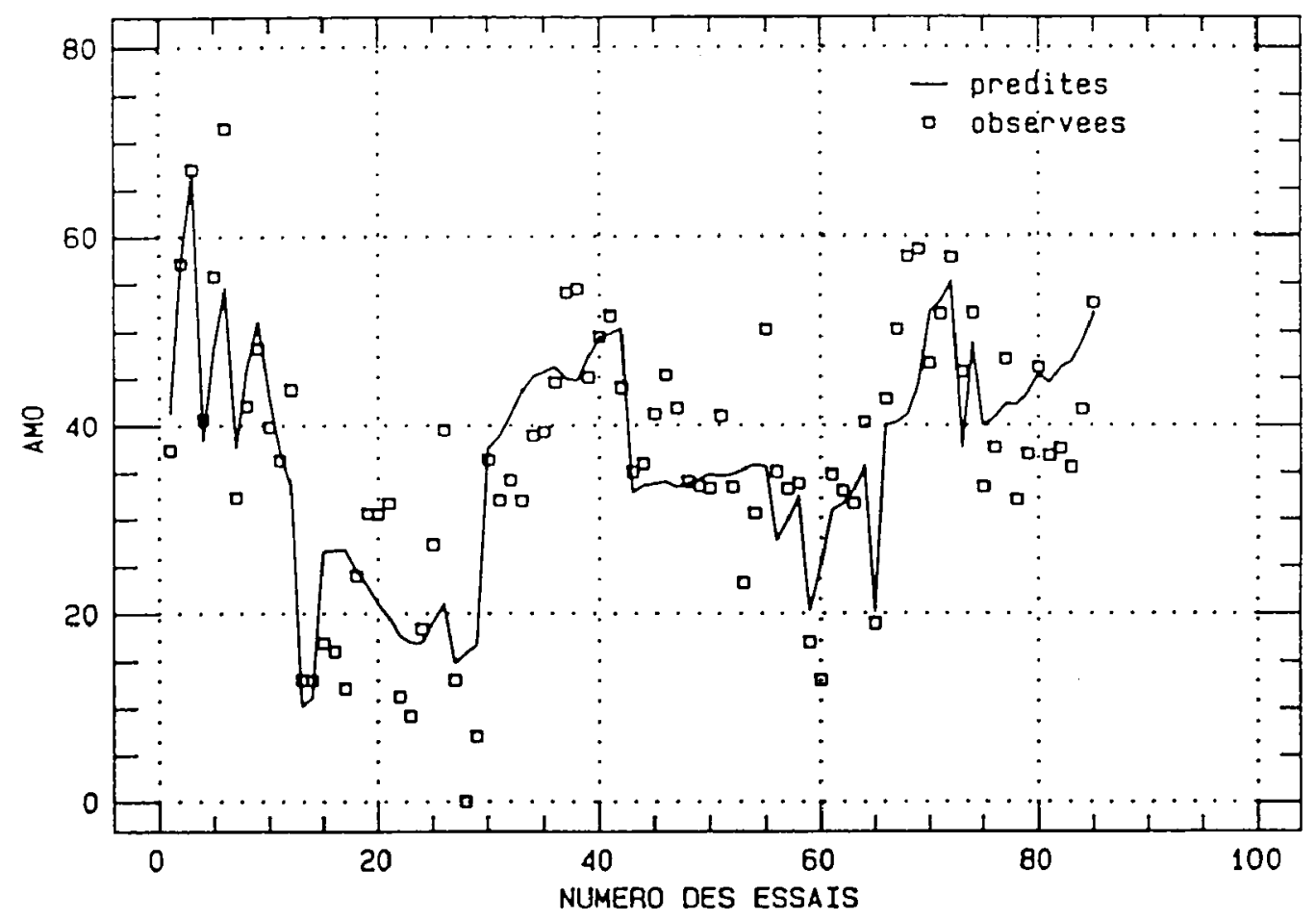

figure 4.2. : Valeurs prédites et observées des taux de mortalités (AMO) en fonction du numéro de référence de l'essai (voir annexe $\mathrm{C}$ ). Régression linéaire sur les turbines FRANCIS.

figure 4.2. : Relationship of predicted and observed transformed mortalities (AMO) with index of experiment (see appendix $\mathrm{C}$ ). Linear regression on FRANCIS turbines.

\subsection{A partir des variables caractérisant les conditions de fonctionnement des roues et les variables composées.}

Le pourcentage de mortalité - ou plus exactement sa transformée AMO - est avant tout relié, parmi l'ensemble des variables caractérisant le fonctionnement hydraulique de la roue, à la vitesse absolue en entrée $V 1(R=0.60)$ et à sa composante giratoire $V 1 u(R=0.58)$ qui lui est très liée. Ces deux variables sont en grande partie déterminées par la hauteur de chute nette (Haut) (coefficient de corrélation supérieur à 0.95). La mortalité est beaucoup moins liée aux composantes normales des vitesses $V 1 \mathrm{~m}(R=0.37)$ et $V 2 m(R=0.25)$, à $V 2(R=0.38)$ ainsi qu'aux vitesses relatives $W 1$ $(R=0.20)$ et $W 2(R=0.39)$. La matrice des corrélations est donnée en annexe $D$.

Parmi les variables composées, (Tou) s'est révélée être la plus lièe à la mortalité, avec un coefficient de corrélation voisin de 0.62 .

Par contre, les liaisons entre $(R A B)(R=0.083$, (Mont) $(R=0.011)$ et la mortalité ne sont pas significatives.

La régression multiple optimale, au sens de régression performante avec le moins de variables, est celle liant $V 1$ et (TOu) à la mortalité; elle explique plus de $70 \%$ de la variance

$$
\mathrm{AMO}=3.12+0.76 \mathrm{~V} 1+0.12 \mathrm{Tou} \quad(\mathrm{R}=0.84)
$$

On a porté sur les figures 4.1. et 4.2. les valeurs observées en fonction des valeurs prèdites par l'expression précédente, ainsi que les valeurs prédites et les valeurs observées en fonction d'un index correspondant au numéro de l'essai référencé dans l'annexe $C$.

Appliquée à des échantillons de cas nouveaux, cette équation de régression reste bonne, dans le sens où les coefficients de corrélation sont très voisins sur les échantillons-tests. Les deux variables intervenant dans la régression sont peu corrélées entre elles et expliquent chacune une proportion comparable de la variance de la mortalité.

On a tenté d'augmenter significativement la part de la variance expliquée en ayant recours à un modèle de régression non linéaire, les exposants de la variable (Tou) ayant été choisis au départ de façon quelque peu arbitraire. L'équation de régression linéaire a été utilisée pour l'estimation initiale des diffèrents paramètres. L'équation finale est la suivante:

$$
A M O=-4.21+1.25 V^{0.821}+2.28 \mathrm{~N}^{0.19}(\mathrm{TL} / \mathrm{esp})^{0.84} \mathrm{~W}^{0.71}
$$

Cette équation explique $75 \%$ de la variance, le coefficient de corrélation $\mathrm{A}$ étant voisin de 


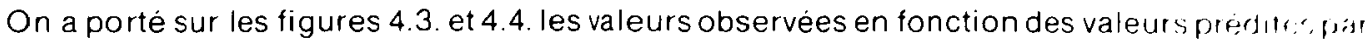
l'expression précédente, ainsi que les valeurs prédites et les valeurs observèes en fonctirsi clurı index correspondant au numéro de l'essai référencé dans l'annexe $C$.

Un seul résidu normé possede une valeur supérieure à 2.5, indiquant une mauvaıs: prér.u.tion. II s'agit d'un essai effectué à Motala en Suède, mortalités observées et prédites ètant respe; tuv;; ment de $0 \%$ et $8.3 \%$.

\section{PRÉDICTION DES MORTALITÉS DANS LES TURBINES KAPLAN}

\subsection{Essais portant sur les juveniles de salmunidés.}

De toutes les variables simples ou nominales, la vitesse de rotation ( $N$ ) est la mieux corrélée a la mortalité $(R=0.52)$. Parmi les variables composées, c'est le rapport de la taille a la distance inter-pales (TL/esp) qui s'est révélée la plus significative ( $R=0.59$ ), suivie par d'autres variables plus ou moins directement liées au rapport de la taille du poisson au diametre de la roue, notamment $(R A B)(R=0.50)$, et (Mont1) $(R=0.42)$.

La mortalité n'est fortement corrélée à aucune variable de vitesse. Les corrélations maximales sont celles avec la vitesse relative $W 1$ en entrée $(R=-0.29)$ et dans une moindre mesure avec la vitesse relative en sortie W2. La matrice des corrélations est donnée en annexe D.

L'équation de régression simple décrivant le mieux la mortalité est:

$$
\mathrm{AMO}=13.41+42.8(\mathrm{TL} / \mathrm{esp}) \quad(\mathrm{R}=0.59)
$$

On a porté sur la figure 4.5. les valeurs observées et les valeurs prédites par l'expression précédente en fonction de la variable (TL/esp).

Seuls deux résidus normés sont au-delà de 2.5 , indiquant des écarts très importants entre prédictions et observations : il s'agit d'essais afférents aux centrales d'Essex $10 \%$ de mortalite observée sur des smolts de $28 \mathrm{~cm}$ ) et de Foster $(31 \%$ de mortalité, valeur de la mortalité la plus élevée observée sur une turbine KAPLAN, obtenue cependant pour des conditions extrèmes de fonctionnement de la roue).

Le recours à un modèle de régression multiple permet de réduire quelque peu la part de I. variance restée inexpliquée.

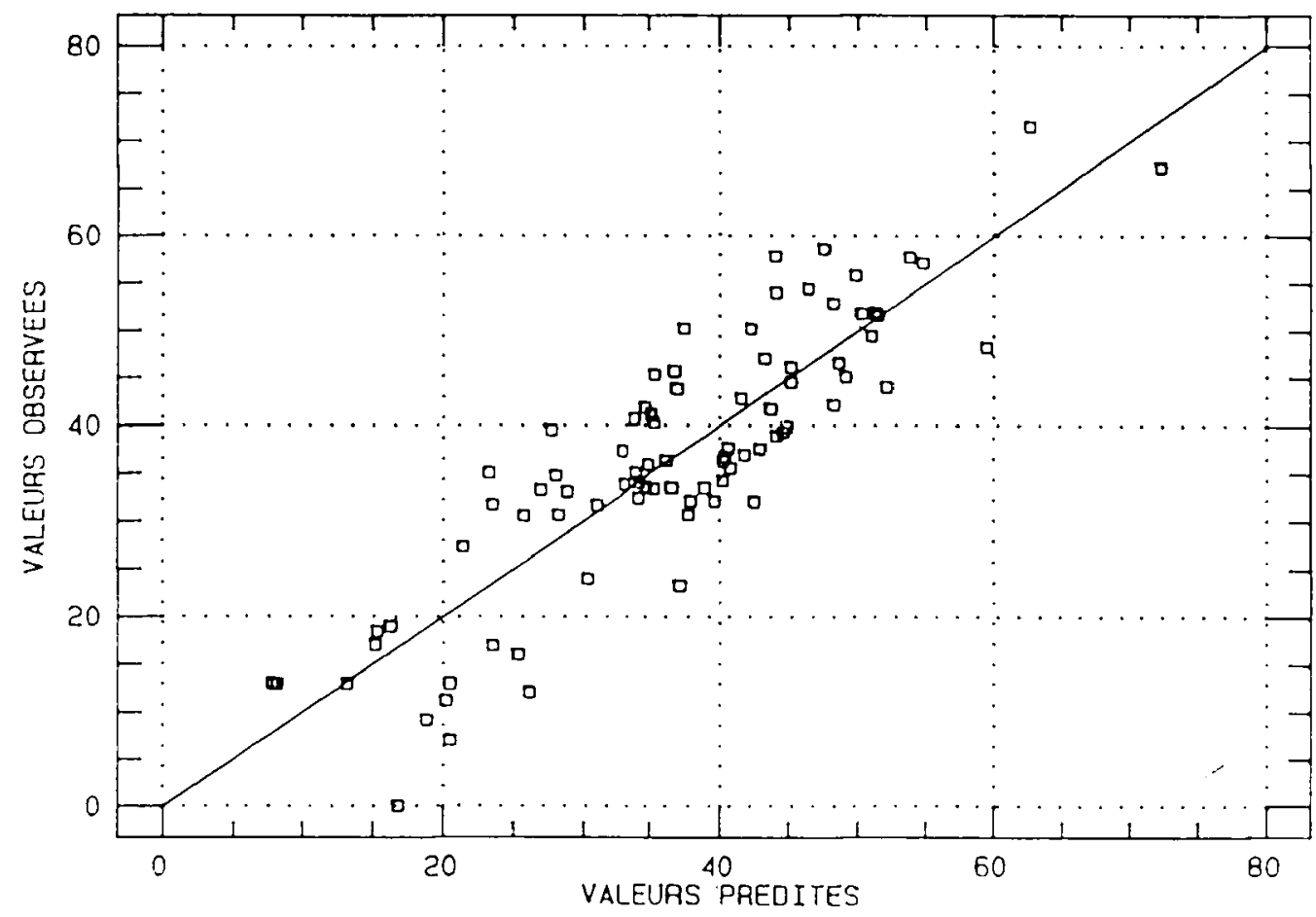

figure 4.3. : Valeurs prédites des taux de mortalité transformés (variable AMO) en fonction des valeurs observées. Régression non linéaire sur les turbines FRANCIS.

figure 4.3. : Relationship of predicted transformed mortalities (AMO) with observed mortalities. Nonlinear regression on FRANCIS turbines. 


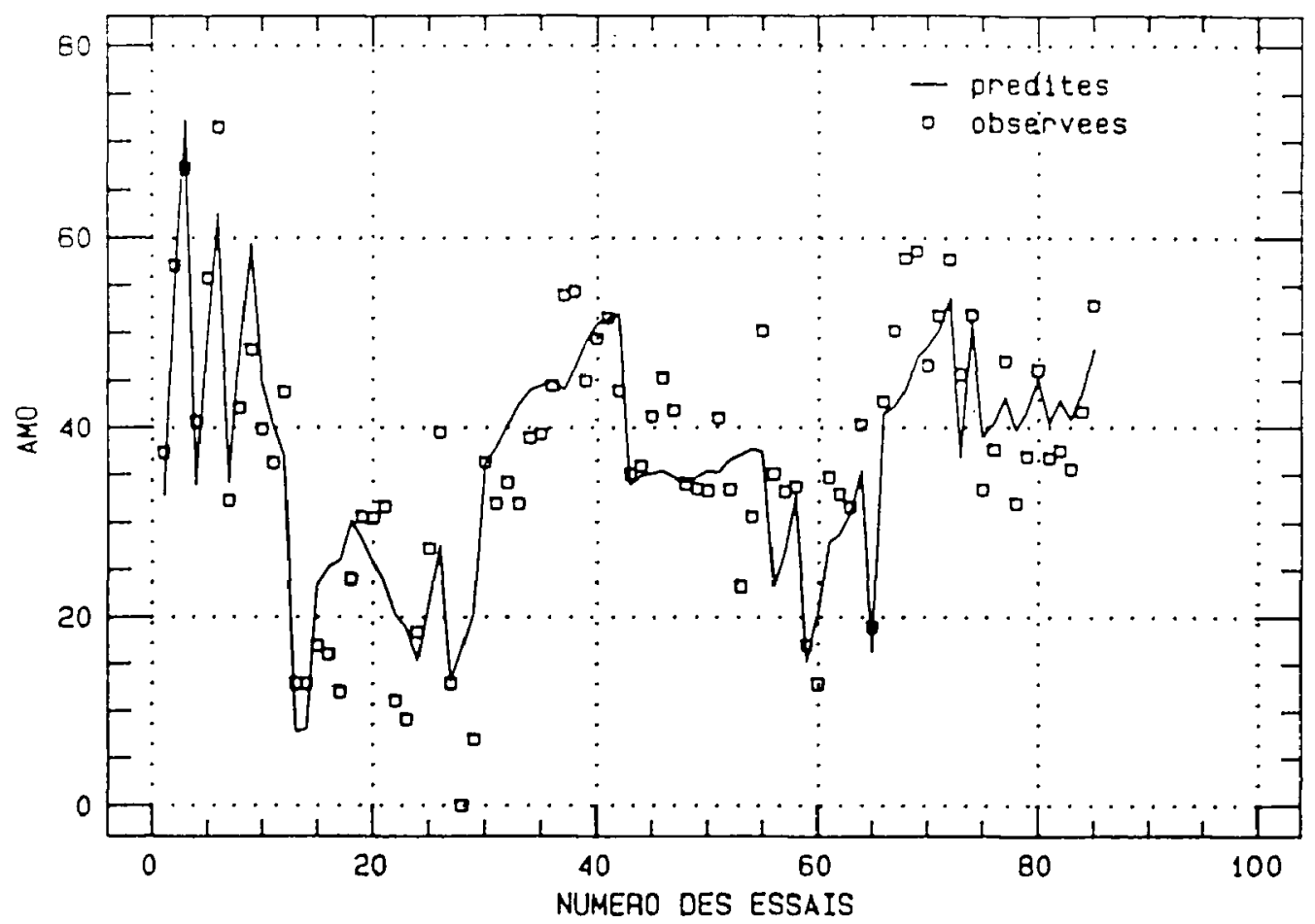

figure 4.4. : Valeurs prédites et observées des taux de mortalités (AMO) en fonction du numéro de référence de l'essai (voir annexe C). Régression non-linéaire sur les turbines FRANCIS.

figure 4.4. : Relationship of predicted and observed transformed mortalities (AMO) with index of experiment (see appendix $\mathrm{C}$ ). Nonlinear regression on FRANCIS turbines.

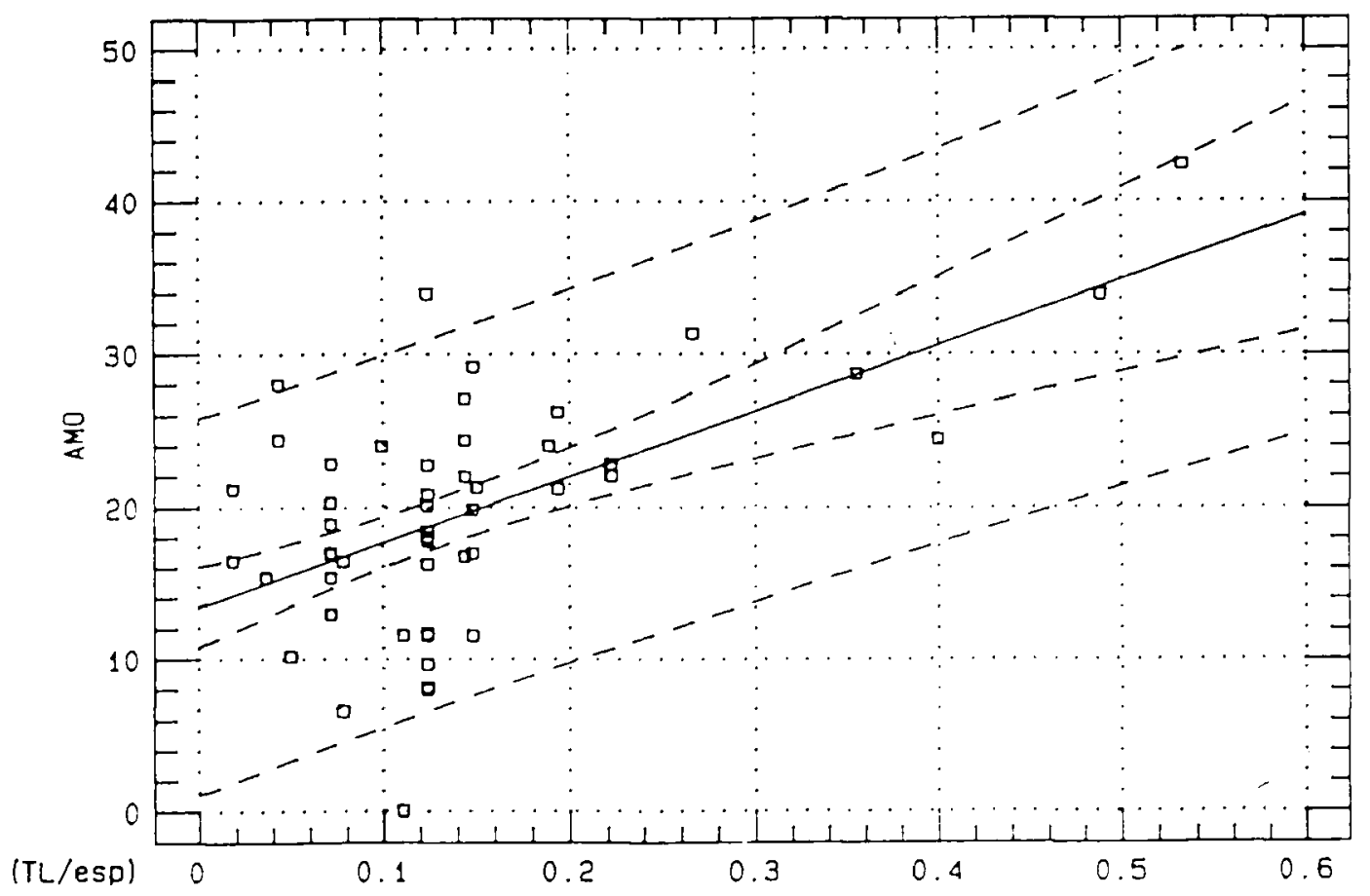

figure 4.5. : Valeurs prédites el observées des taux de mortalité transformés (variable AMO) sur les salmonidés en fonction de la variable (TL/esp). Règression linèaire sur les turbines KAPLAN avec les intervalles de confiance et de prédiction à $95 \%$.

figure 4.5. : Relationship of predicted and observed transformed mortalities (AMO) on salmonids with the variable (TL/esp). Linear regression on KAPLAN turbines with the $95 \%$ confidence and prediction limits. 
La meilleure régression est celle faisant intervenir le paramètre (TL/esp) et la puissance (Puis) exprimée en MW

$$
\mathrm{AMO}=10.6+53(\mathrm{TL} / \mathrm{esp})+0.083 \text { Puis } \quad(R=0.65)
$$

L'apport de la puissance dans la régression est cependant d'un intérêt limité ; cette variable n'intervient pratiquement que pour expliquer la mortalite plus importante que la moyenne $(17 \%$ et $22 \%$ ) constatée sur les deux essais afférents à la grosse turbine de Lower Monumental.

Les fractions de la variance de la mortalité expliquées par les deux modèles précédents sont respectivement de $35 \%$ et $42 \%$. Si on élimine les deux tests dont les résidus normés ont une valeur élevée, cette part de la variance passe à plus de $52 \%$.

\subsection{Essais portant sur les anguilles.}

Le facteur le plus corrélé à la mortalité est le même que pour les juvéniles de salmonidés, c'est-à-dire le rapport (TL/esp). La matrice des corrélations est donnée en annexe D.

La formule de régression simple est :

$$
A M O=28.6+48.7 \text { (TL/esp) } \quad(R=0.85)
$$

On a porté sur la figure 4.6. les valeurs observées et les valeurs prédites par l'expression précédente en fonction de la variable (TL/esp).

Un seul essai présente un résidu normé supérieur à 2.5. Surces mêmes données, la technique de régression linéaire multiple "pas à pas" n'a pas permis de sélectionner un modèle de régression comportant une autre variable que (TL/esp)

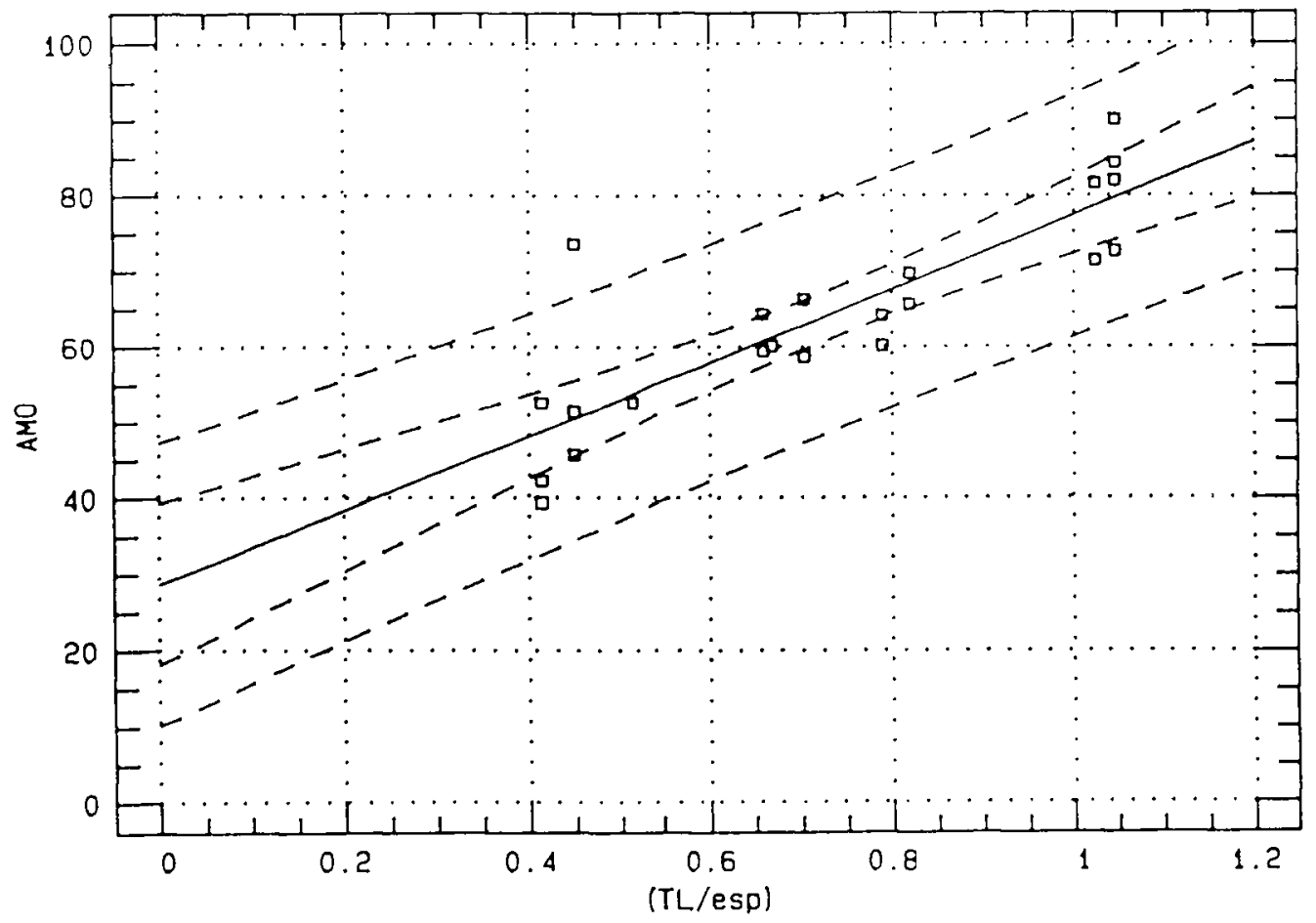

figure 4.6. : Valeurs prédites et observées des taux de mortalité transformés (variable AMO) sur les anguilles en fonction de la variable (TL/esp). Régression linéaire sur les turbines KAPLAN avec les intervalles de confiance et de prédiction à $95 \%$.

figure 4.6. : Relationship of predicted and observed transformed mortalities (AMO) on eels with the variable (TL/esp). Linear regression on KAPLAN turbines with the $95 \%$ confidence and prediction limits.

\subsection{Essais sur les salmonidés et les anguilles confondus}

Les régressions effectuées indépendamment sur les juvéniles de salmonidés et les anguilles ont mis en évidence la même variable explicative et des équations de régression relativement voisines. 

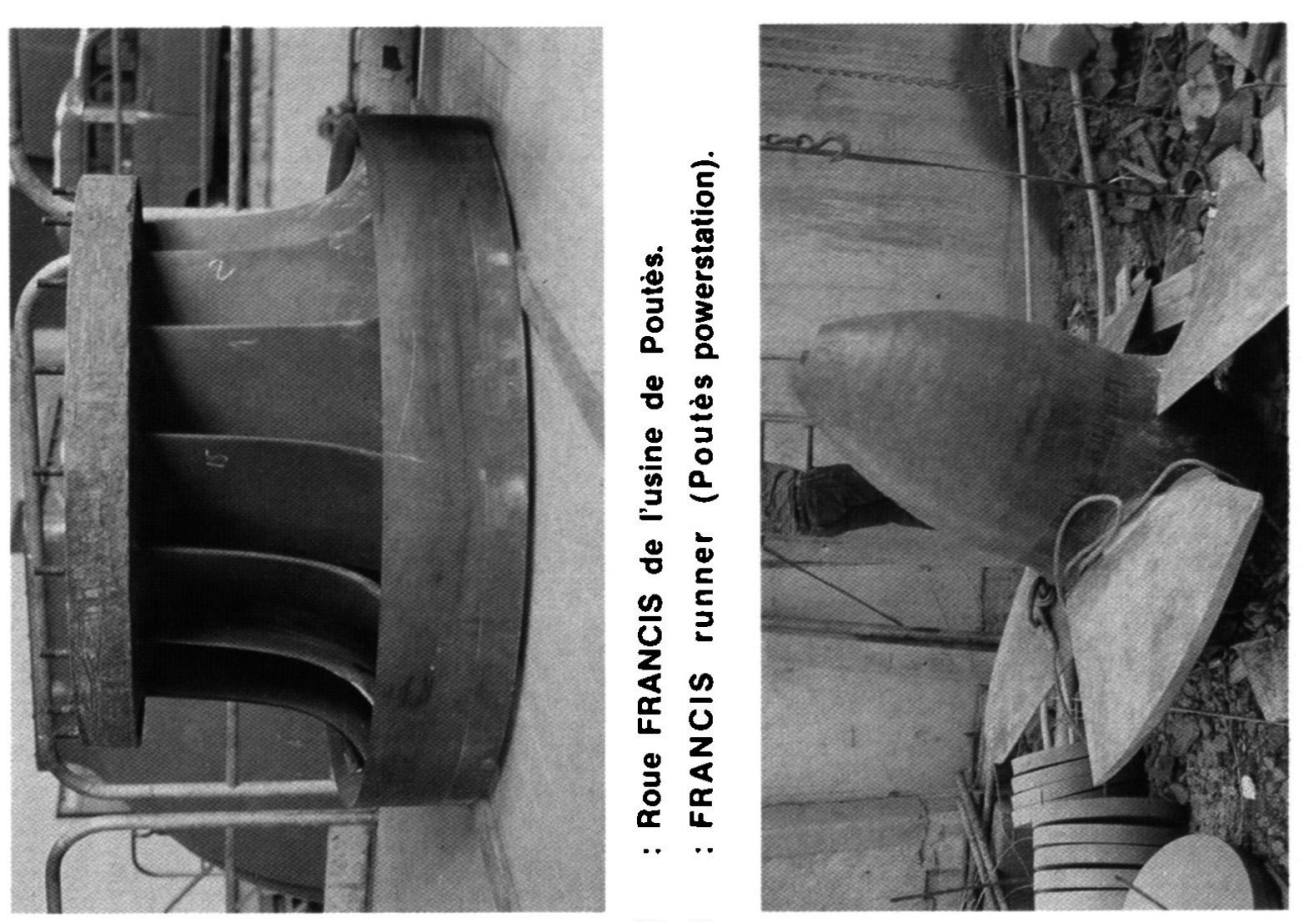

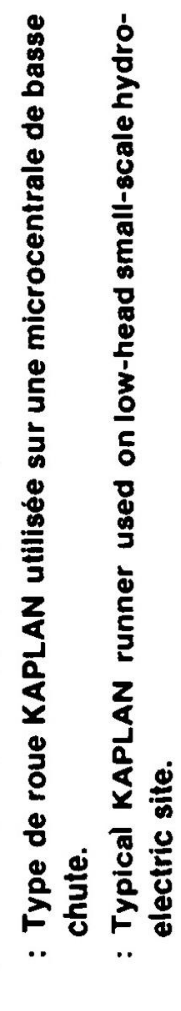

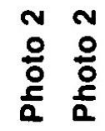

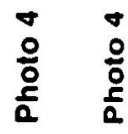
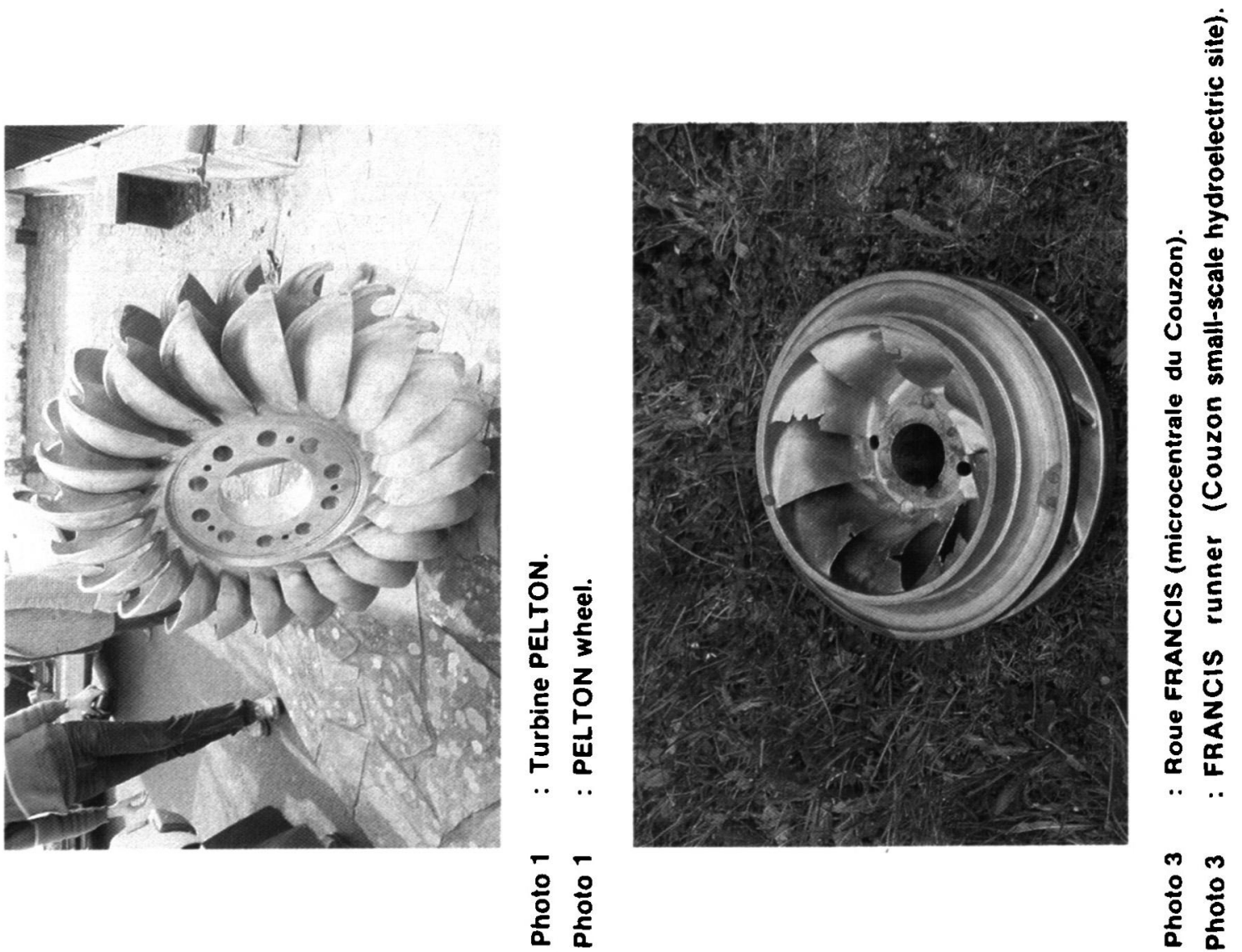

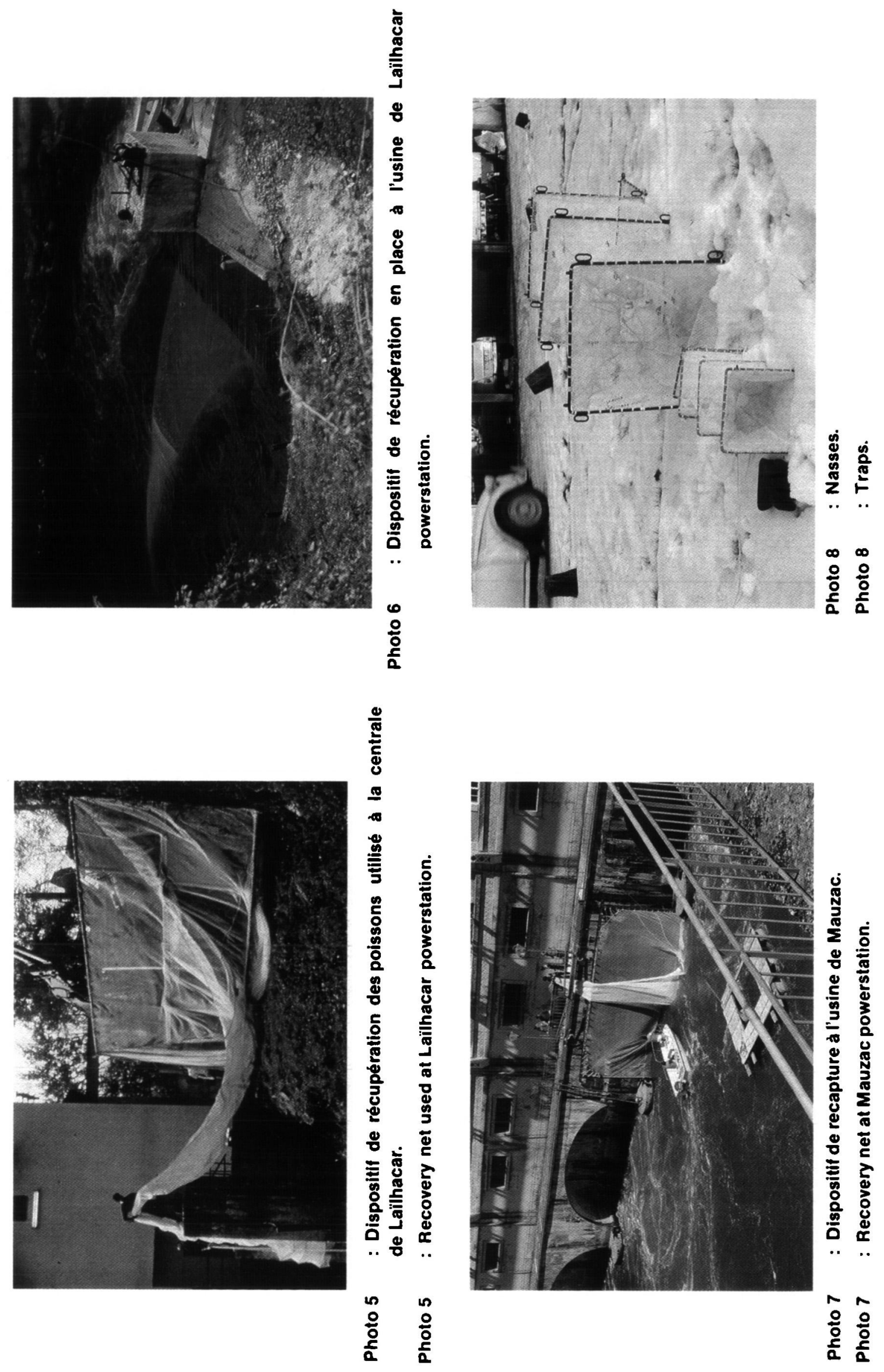

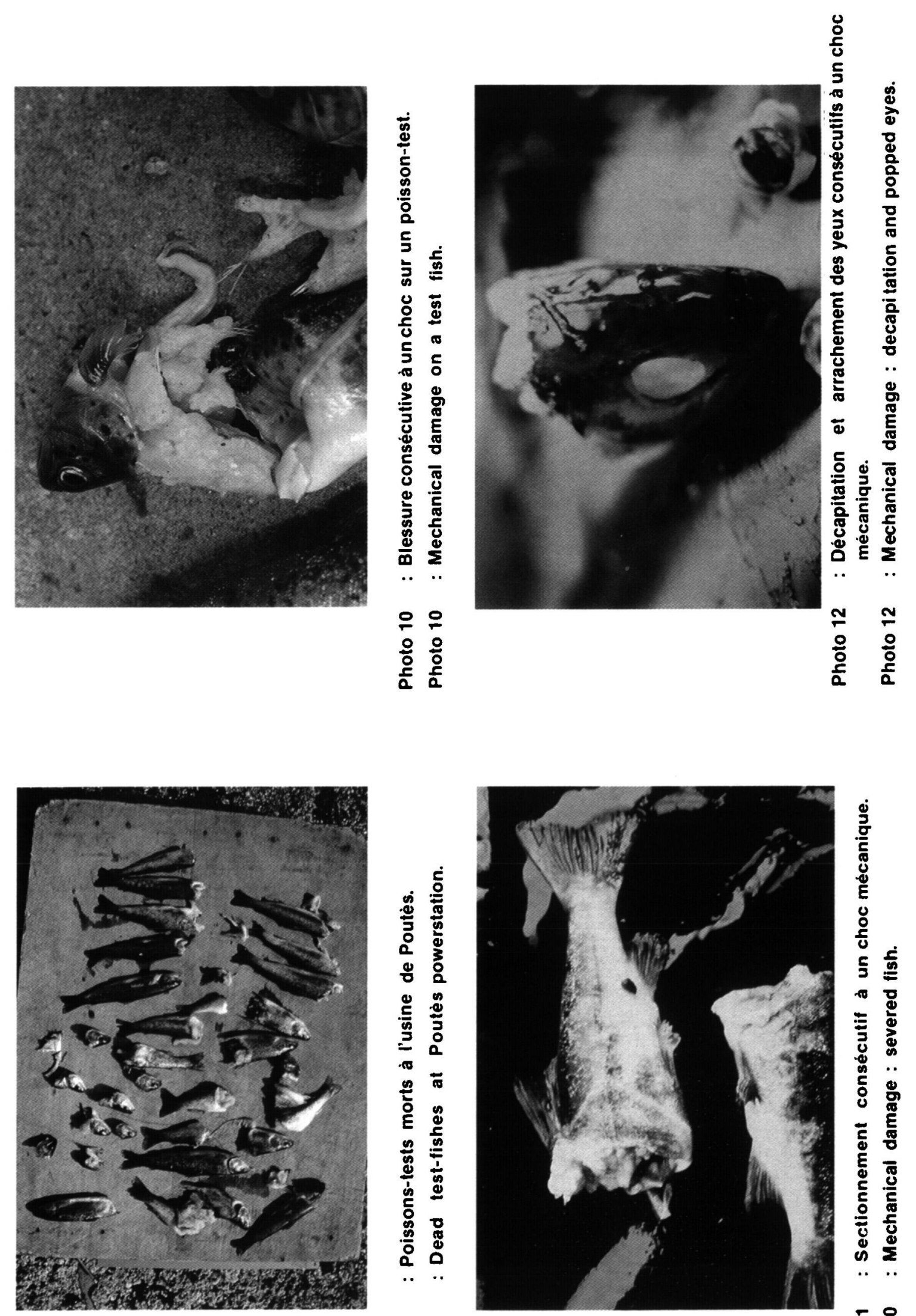

$\begin{array}{ll}0 & 0 \\ \circ & 0 \\ ㅇ & 0 \\ & \frac{1}{2} \\ \frac{1}{2} & \frac{1}{a}\end{array}$

$=$ 으

$\frac{\frac{0}{2} \frac{2}{2}}{2}$ 

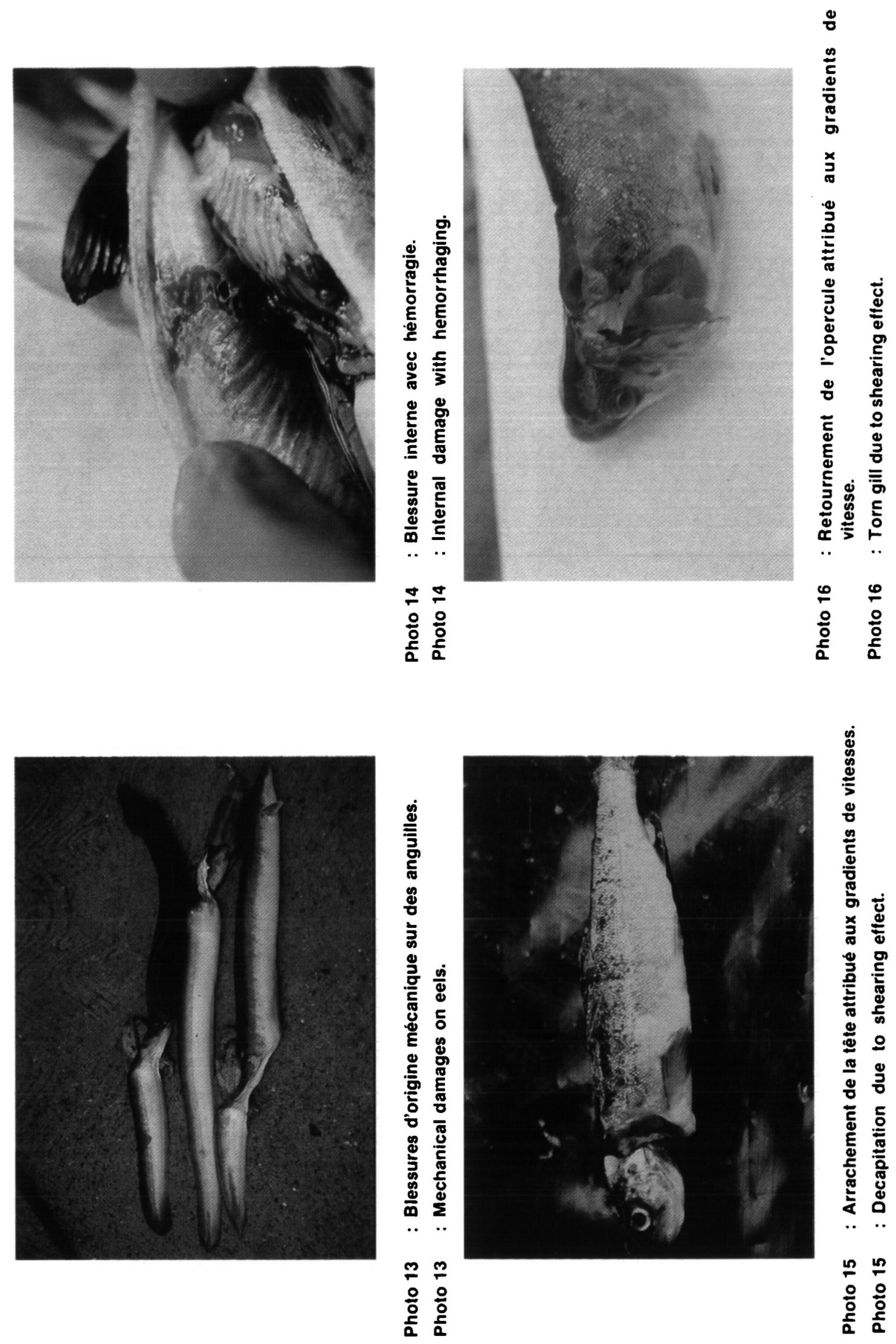
En effectuant un traitement sur l'ensemble des essais, juvéniles de salmonidés et anguilles confondus, on retrouve évidemment comme principale variable (TL/esp) qui explique près de $90 \%$ de la variance totale. La matrice des corrélations est donnée en annexe $D$.

L'équation de régression simple est la suivante :

$$
\text { AMO }=11.1+68.7(\mathrm{TL} / \mathrm{esp}) \quad(\mathrm{R}=0.94)
$$

On a porté sur les figures 4.7. et 4.8. les valeurs observées et les valeurs prédites par l'expression précédente en fonction de la variable (TL/esp), ainsi que les valeurs prédites et les valeurs observées en fonction d'un index correspondant au numéro de l'essai référencé dans l'annexe $C$ (les essais afférents aux anguilles ont été mis à la suite des essais sur les salmonidés).

En éliminant les trois mêmes tests qui déjà lors des traitements séparés s'ajustaient mal aux équations (résidus normés supérieurs à 2.5), la variance expliquée passe à plus de $93 \%$, l'équation de régression demeurant très voisine de la précédente:

$$
\mathrm{AMO}=10.7+68.8 \text { (TL/esp) } \quad(\mathrm{R}=0.96)
$$

Par la méthode "pas à pas", la meilleure combinaison significative de variables qui régressent la mortalité est celle constituée par (TL/esp), TL, et la puissance (Puis). Avec un coefficient de corrélation voisin de 0.98 , ce modèle régresse près de $96 \%$ de la variance.

$$
\mathrm{AMO}=7.4+57.7(\mathrm{TL} / \mathrm{esp})+20.25 \mathrm{TL}+0.089 \text { Puis } \quad(R=0.98)
$$

La variante (Puis), même si son apport à la régression est significative, n'apporte qu'une contribution négligeable $(1 \%)$ à la part de la variance expliquée.

Le résidu afférent à la régression simple restant lié à $T L$ et esp, on a procédé à un ajustement par une régression non linéaire expliquant plus de $91 \%$ de la variance :

$$
\mathrm{AMO}=11.3+74.3\left(\mathrm{TL} \cdot 074 / \mathrm{esp}^{0.768}\right) \quad(\mathrm{R}=0.955)
$$

En laissant de côté les trois mêmes tests s'ajustant mal aux différentes équations de régression, la part de la variance expliquée passe à plus de $94 \%$ :

$$
\text { AMO }=12.2+72.7\left(\mathrm{TL}^{\left.1.125 / \mathrm{esp}^{0.843}\right) \quad(R=0.97)}\right.
$$

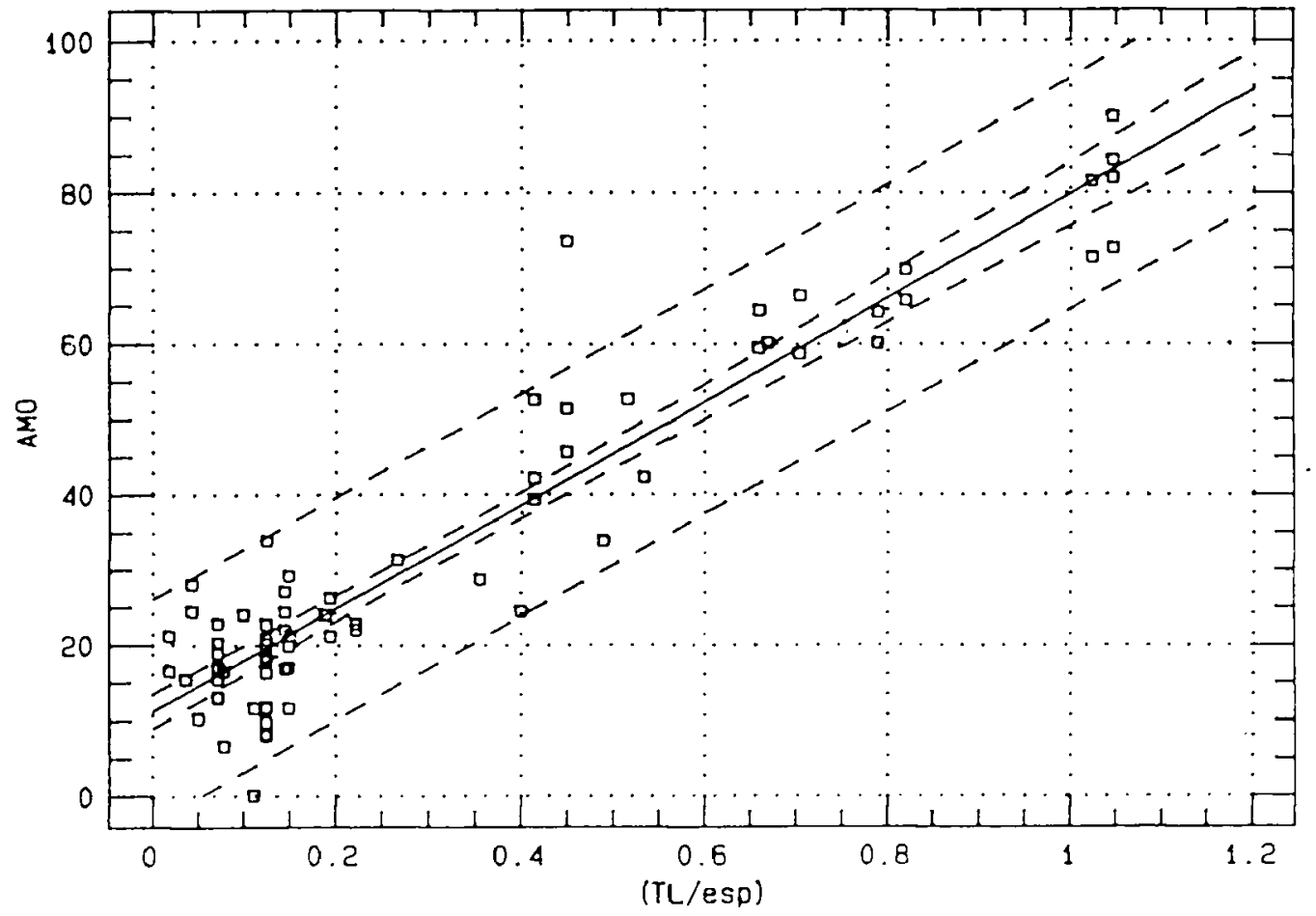

figure 4.7. : Valeurs prédites et observèes des taux de mortalité transformés (variable AMO) sur les salmonidés et les anguilles en fonction de la variable (TL/esp). Régression linéaire sur les turbines KAPLAN avec les intervalles de confiance et de prédiction a $95 \%$.

figure 4.7. : Relationship of predicted and observed transformed mortalities (AMO) on salmonids and eels with the variable (TL/esp). Linear regression on KAPLAN turbines with the $95 \%$ confidence and prediction limits. 


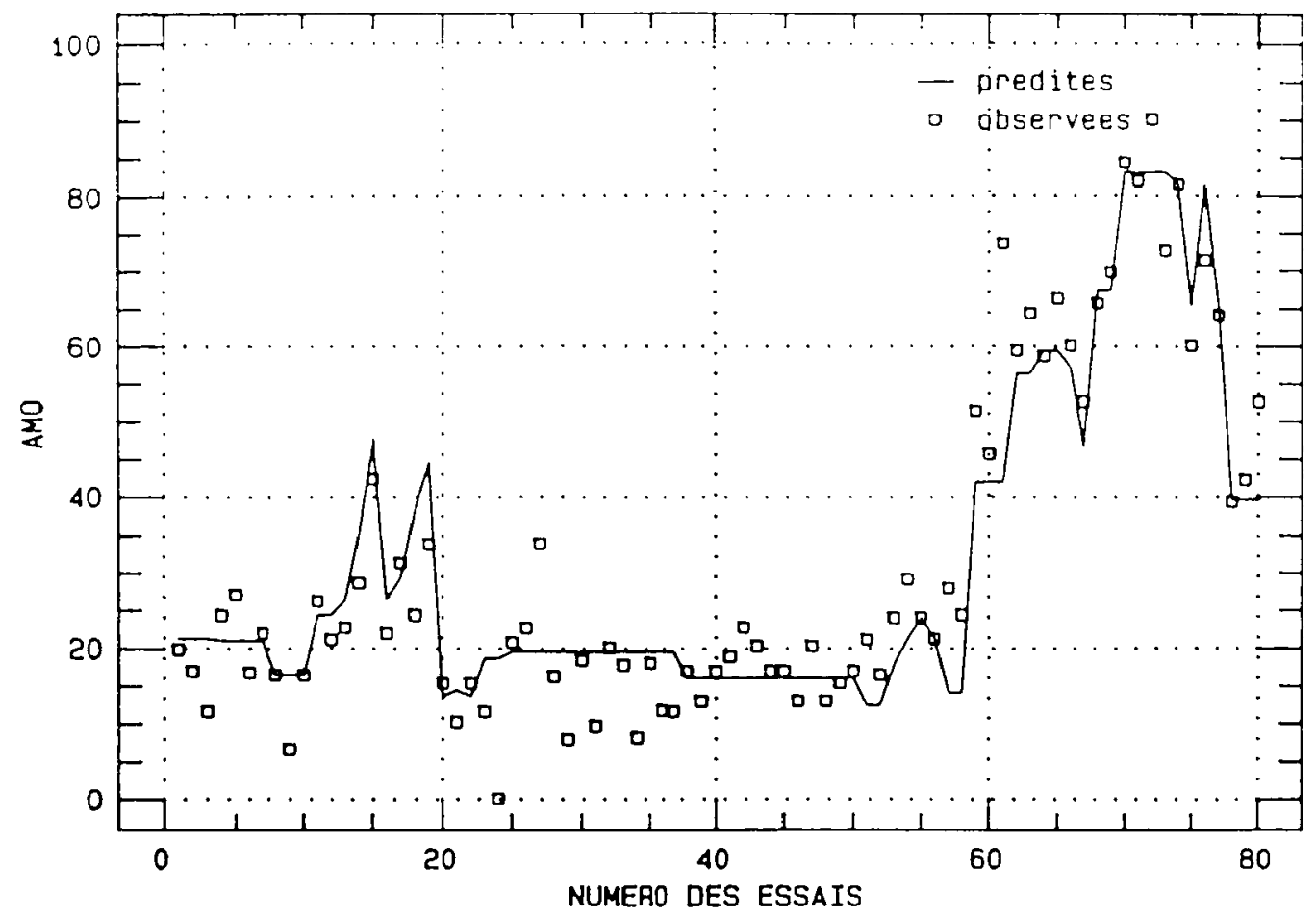

figure 4.8. : Valeurs prédites et observées des taux de mortalités (AMO) sur les salmonidés et les anguilles en fonction du numéro de référence de l'essai (voir annexe C). Régression linèaire sur les turbines KAPLAN.

figure 4.8. : Relationship of predicted and observed transformed mortalities (AMO) on salmonids and eels with index of experiment (see appendix $C$ ). Linear regression on KAPLAN turbines.

\section{DISCUSSION}

\subsection{Turbines FRANCIS}

L'analyse des données sur les turbines FRANCIS a mis en évidence l'influence sur les mortalités de deux facteurs relativement peu liés entre eux :

- d'une part la vitesse absolue $V 1$ (ou sa composante périphèrique $V 1 u$ en entrée de roue qui lui est fortement corrélée).

- d'autre part un groupement de variables où interviennent la vitesse relative W1, la taille du poisson (TL), l'espace inter-aube (esp) et dans une moindre mesure la vitesse de rotation $N$.

Le fait que les dommages soient liés à la vitesse absolue en entrée de roue, et par conséquent à ia chute qui lui est très corrélée, n'est pas étonnant. Ce résultat peut être rapproché des expérimentations effectuées sur les poissons immergés dans des jets à fortes vitesses (voir II, paragraphe 5), pour lesquels les dommages attribués au cisaillement devenaient notables dès que les vitesses atteignaient une vingtaine de $\mathrm{m} / \mathrm{s}$ puis croissaient avec la vitesse.

En cela nous ne partageons pas l'opinion de certains auteurs, qui, encore très récemment (EICHER, 1987), ne considérent pas la chute comme un facteur déterminant l'importance des dommages, en se basant sur le fait que des niveaux de mortalités voisins ont été observés sur des turbines fonctionnant sous des chutes très différentes (Willamette Falls et Shasta, respectivement sous $12 \mathrm{~m}$ et $130 \mathrm{~m}$ ). Cette assertion nous parait un peu rapide, car il semble difficile de mettre en évidence l'influence - ou plutôt la non-influence - de la chute sur la mortalité en raisonnant sur des turbines dont les diamètres (respectivement $0.90 \mathrm{~m}$ et $4.65 \mathrm{~m}$ ) et les vitesses de rotation (respectivement $240 \mathrm{trs} / \mathrm{mn}$ et $138 \mathrm{trs} / \mathrm{mn}$ ) sont radicalement différentes.

Le second facteur explicatif de la mortalité, que ce soit la variable (Tou), ou bien le groupement des variables (TL/esp), $N$, et $W 1$, intervenant dans la régression non linéaire, sont indicatrices et cela sans ambiguïté possible, de dommages d'origine mécanique. Une certaine fraction des dommages liés aux gradients de vitesse en entrée de roue doit aussi être expliquée grâce à cette variable: à valeur de $V 1$ donnée, les gradients transversaux et longitudinaux des vitesses. et par conséquent les dommages liés à ces gradients, dans la mesure où ils existent, seront une fonction décroissante du diamètre de la roue; cette variabilité des gradients à vitesse d'entrée constante ne peut être prise en compte que par le second groupe de variables explicatives. 


\subsection{Turbines KAPLAN}

La variable expliquant le mieux les mortalités est le rapport de la taille du poisson à l'espace inter-pales mesuré à mi-pale. Si l'on considère l'ensemble des essais, la mortalité est liée aux dimensions relatives du poisson et de la roue mais est indépendante des conditions hydrauliques régnant dans cette roue, en particulier des vitesses absolues $V 1$ et relatives $W 1$ en entrée.

On retrouve donc une observation effectuée par certains auteurs, à savoir que la fraction la plus importante des dommages était d'origine mécanique et qu'elle dépendait relativement peu des conditions de fonctionnement de la turbine.

Il faut se garder cependant de considérer la mortalité comme uniquement de nature mécanique (chocs, sectionnement). Les autres formes de mortalité, si elles n'ont pas pu être mises en évidence. ne sont pas exclues pour autant. On rappelle que plus de $50 \%$ de la variance sur les mortalités des juvéniles de salmonidés reste inexpliquée. La variabilité sur les vitesses en entrée de roue sur l'ensemble des essais étant beaucoup moins forte sur les turbines KAPLAN que sur les FRANCIS, il n'est pas étonnant que les dommages éventuels causés par les gradients de vitesses soient plus difficiles à degager, leur importance devant rester du même ordre de grandeur et relativement faibles - de quelques pour cent — sur la majoritè des essais.

Le fait qu'il ait été impossible de mettre en évidence l'influence des conditions de vitesse en entrée de roue sur les mortalités peut avoir une autre explication: on a noté, dans la première partie, qu'il existait une différence fondamentale entre les champs des vitesses dans les roues FRANCIS et KAPLAN : dans les turbines FRANCIS, les vitesses absolues ou relatives en entrée sont relativement homogènes sur toute la hauteur du bord d'attaque de l'aube, ces vitesses variant notablement suivant l'ouverture des directrices; par contre, dans les turbines KAPLAN, les vitesses absolues en entrèe augmentent de la péripherie vers le moyeu, alors que les vitesses relatives, à l'inverse, augmentent fortement du moyeu à la périphérie. Cette hétérogénéité des vitesses d'un point à l'autre de la roue est beaucoup plus importante que la variation de ces mêmes vitesses en un point donné résultant de l'ouverture ou de la fermeture de la roue. D'où une interprétation de l'impossibilité à expliquer les mortalités en ayant recours aux triangles des vitesses à mi-pale; on peut cependant difficilement faire autrement dans la mesure où il est a priori impossible de déterminer sur un essai donné si le poisson a abordé la roue près du moyeu ou au contraire à la périphérie. Uneanalyse par régression a été effectuée en considérant à la fois les triangles de vitesse à la périphérie, au moyeu ainsi qu'à mi-pale; elle n'a rien ajoutè à l'analyse initiale effectuée à partir des triangles des vitesses calculés à mi-pale.

Lors de certaines expérimentations, l'influence du régime de fonctionnement de la turbine a été mise en évidence. Sur le Neckar, BERG (1985) a obtenu une relation entre la mortalité et l'angle (Bet1) que fait la direction des filets fluides avec le plan de la roue en entrée, angle voisin de celui des pales. La mortalité sur les anguilles augmentait significativement au fur et à mesure de la fermeture de la roue: Iors d'une réduction de débit, l'angle que font les pales avec le plan de la roue diminue et parallèlement l'espace offert au poisson se trouve limité.

Le fait que ce facteur n'ait pu ètre mis en évidence sur l'ensemble des essais analysès, en particulier sur ceux afférents aux anguilles, n'est pas étonnant: sur la majorité des installations. seuls deux régimes de fonctionnement ont été testés, ce qui est nettement insuffisant pour espérer dégager des résultats statistiques significatifs sur une influence éventuelle du régime. 


\section{CONCLUSIONS}

Le plus grand nombre des expérimentations sur le transit des poissons à travers les turbines a porté sur des salmonidés. Les diverses espèces de salmonidés ne semblent pas être affectées de façon significativement différente.

Par contre, la plupart des autres espèces, en particulier les clupéidés, paraissent beaucoup plus fragiles que les salmonidés, les mortalités observées étant de 2 à 3 fois plus importantes. Les mortalités, généralement très fortes, constatées sur les anguilles, peuvent s'expliquer par leur taille.

Les dommages ont été classés en trois catégories, suivant leur origine probable: les dommages d'ordre mécanique, ceux attribués au cisaillement et enfin ceux consécutifs à l'exposition du poisson à des pressions très rapidement variables ou à la cavitation. Si les dommages induits par les chocs mécaniques sont relativement faciles à distinguer, l'identification des autres causes de mortalité, comme l'effet de la pression, de la cavitation ou celui des gradients transversaux ou longitudinaux de vitesse est relativement subjective. Un même type de dommage (décapitation. yeux arrachés...) peut être attribué, suivant l'auteur, à des causes toutes différentes.

Des expérimentations ont montré que les poissons tolèrent sans dommages les mises en pression se produisant en amont des turbines. L'effet éventuel de la dépression brutale subie lors du transit dans la roue, en particulier sur la vessie natatoire, dépend de l'importance relative de cette dépression, par rapport à la pression à laquelle est adapté l'organisme du poisson, et de la vitesse à laquelle s'effectue cette dépression.

Tant que la réduction relative de pression reste inférieure à 50-60\% de la pression d'adaptation initiale, les risques demeurent limités.

Dans le cas d'une prise d'eau de fond, dans l'hypothèse où l'organisme du poisson est adapté à la pression correspondant à la profondeur de celle-ci, la brusque dépression au passage de la roue, voire le simple retour à la pression atmosphérique dans le canal de fuite, pourra entraîner des dommages considérables.

Pour des poissons migrant dans les couches de surface - et donc adaptès à une pression voisine de la pression atmosphérique - les dépressions maximales (en dessous de la pression atmosphérique) admissibles dans la turbine ou dans l'aspirateur sans risques de dommages sont de l'ordre de $5 \mathrm{~m}$ à $6 \mathrm{~m}$ d'eau, soit 0.5 à 0.6 atmosphère. Certaines expérimentations ont d'ailleurs mis en évidence l'influence de la hauteur de suspension de la roue ou du coefficient de Thoma, qui déterminent l'importance de la dépression en sortie de roue, sur le taux de mortalité.

L'effet possible du cisaillement a été confirmé en laboratoire en introduisant des poissons dans ou à la périphérie de jets à fortes vitesses: les dommages deviennent notables dès que les vitesses atteignent des valeurs de l'ordre de $20 \mathrm{~m} / \mathrm{s}$. L'effet des gradients longitudinaux des vitesses, en particulier lors du transit dans des roues de petites dimensions, n'est pas à exclure.

A l'issue des expérimentations effectuées aussi bien en France qu'à l'étranger, il est possible de dégager un certain nombre de points sur les dommages subis par les poissons lors de leur transit à travers les turbines:

- il ne semble pas exister de différence significative sur les niveaux de mortalités atteints dans les divers types de turbines à réaction - FRANCIS ou KAPLAN - à condition évidemment que ces turbines fonctionnent sous des chutes comparables et qu'elles aient des diamètres et des vitesses de rotation voisines. Si les mortalités moyennes observées sur les turbines FRANCIS $(37 \%)$ sont plus importantes que celles observees sur les turbines KAPLAN $(9 \%)$, ce ne peut ètre que parce que les premières sont installées sur des chutes plus importantes.

- il est certain que l'importance de la chute détermine en partie l'ordre de grandeur des dommages. la chute déterminant par ailleurs l'intensité des vitesses et en partie celle de leurs gradients en entrée de roue.

- quel que soit le type et les caractéristiques d'une turbine, un pourcentage minimal de mortalité de $3 \%$ à $5 \%$ semble inévitable.

- sur les turbines KAPLAN, les dommages les plus fréquemment observés sont ceux consécutifs à des chocs contre les pales de la roue.

- sur les turbines FRANCIS, la distribution des dommages est très variable suivant le site testè et, sur un même site, suivant les conditions de fonctionnement de la turbine. Les mortalités attribuées aux gradients de vitesse peuvent représenter une part importante des dommages constatès.

- la taille du poisson relative à la dimension de la turbine ou à l'espacement entre aubes ou pales de la roue semble être un paramètre important. Il suffit de comparer sur une installation donnée les mortalités afférentes à des poissons de tailles très différentes. L'influence de la taille du poisson ne semble cependant très significative que sur les dommages d'ordre mécanique.

- certains auteurs ont mis en évidence la similitude des courbes de survie et des courbes de rendement hydraulique de la turbine. Cette observation ne peut cependant être prise comme une 
règle générale: les dommages les plus faibles sur certaines turbines FRANCIS ont de façon indubitable èté constatés dans des conditions de fonctionnement très éloignées des conditions optimales.

Les observations effectuées lors des expérimentations sur la nature de certaines blessures et leur origine possible, comme les gradients longitudinaux ou transversaux de vitesse ont amené les auteurs à tenter de prendre en considération dans une analyse les conditions de vitesse absolue et relative régnant dans la roue au moment du passage du poisson. mi-pale.

Les triangles des vitesses ont été déterminés en entrée et en sortie de roue à mi-aube et

L'analyse des données sur les turbines FRANCIS a mis en évidence l'influence sur les mortalités de deux facteurs relativement peu liés entre eux:

- d'une part la vitesse absolue $V 1$ en entrée de roue (qui est fortement corrélée à la hauteur de chute).

- d'autre part un groupement de variables où interviennent la vitesse relative $W 1$, la taille du poisson (TL), l'espace inter-aube calculé à mi-aube (esp) ainsi que la vitesse de rotation (N).

La meilleure régression prédictive du pourcentage de mortalité - ou plus exactement de sa variable transformée AMO - sur les turbines FRANCIS, est la suivante :

$$
A M O=-4.21+1.25 V 1^{0.821}+2.28 N^{0.19} \text { (TL/esp) }{ }^{0.84} W_{10.71}
$$

Sur les turbines KAPLAN, l'influence des variables hydrauliques est moins nette, le rapport de la taille du poisson à l'espacement inter-pales calculé à mi-pale se révèle être le meilleur indicateur des dommages potentiels :

$$
\mathrm{AMO}=10.7+68.8(\mathrm{TL} / \mathrm{esp})
$$

Les modèles précédents peuvent être utilisès pour obtenir des ordres de grandeur des mortalités sur des salmonidés et des anguilles. Ils ne sont pas adaptés aux autres espèces, en particulier aux juvéniles de clupéidés sur lesquels, jusqu'à preuve du contraire, les dommages s'avèrent beaucoup plus importants, ainsi qu'aux espèces physoclistes, plus sensibles aux variations de pression que les espèces physostomes comme les salmonidés.

Les modèles prédictifs proposés pourront être aisément améliorés et affinés au fur et à mesure que deviendront disponibles les résultats afférents à de nouvelles expérimentations. 


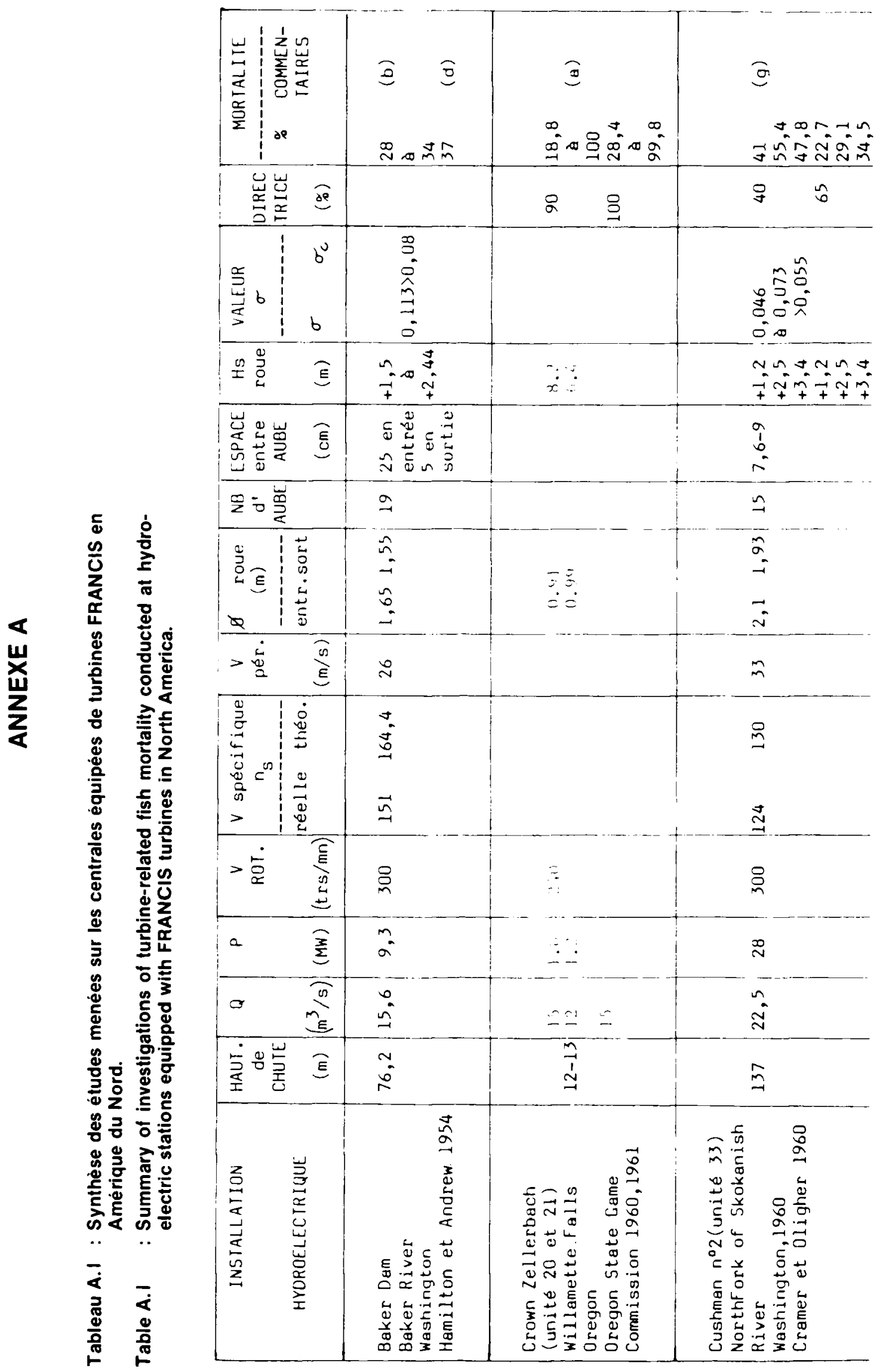




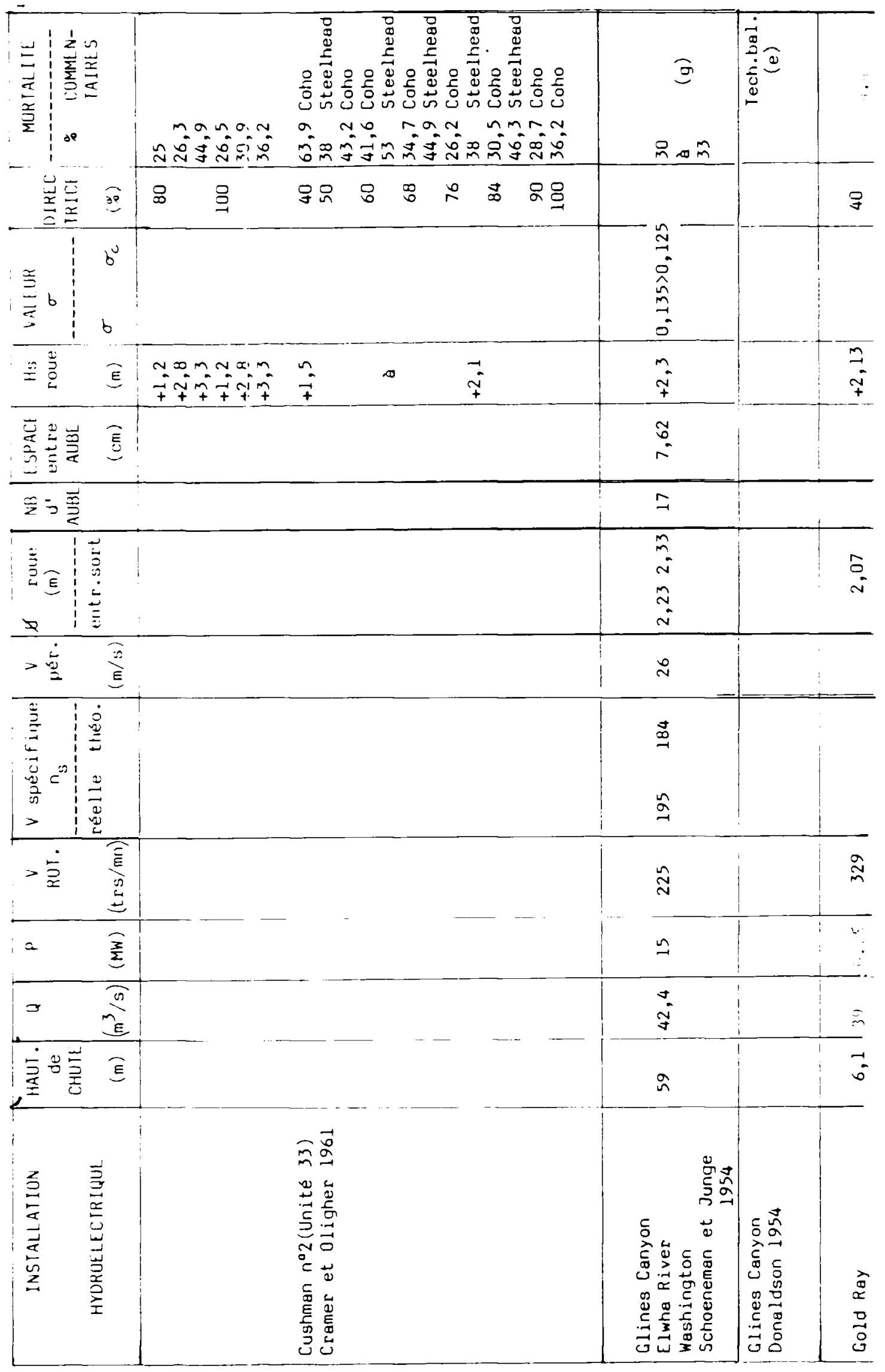




\begin{tabular}{|c|c|c|c|c|c|c|}
\hline 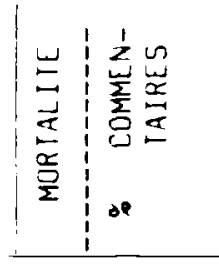 & @ & $\begin{array}{l}\infty \\
j \\
j\end{array}$ & 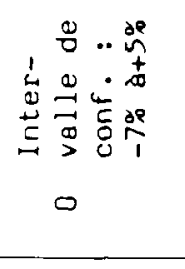 & 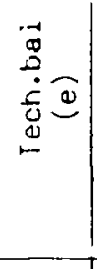 & $\begin{array}{l}\because \\
\vdots \\
0 \\
0\end{array}$ & 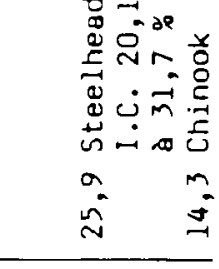 \\
\hline 岸岂岂 & & 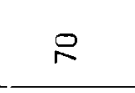 & & & & $\infty$ \\
\hline \begin{tabular}{c|c}
$\cong$ & $b$ \\
$\sum^{\prime}$ & $b$ \\
& $b$
\end{tabular} & & & $\begin{array}{l}\stackrel{0}{a} \\
- \\
0 \\
0 \\
0 \\
0 \\
0\end{array}$ & & & \\
\hline 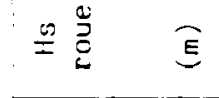 & & & & & & \\
\hline 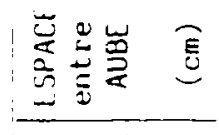 & & & $\tilde{n}$ & & & \\
\hline$\sum=\frac{\Phi}{2}$ & & & $\cong$ & & & \\
\hline 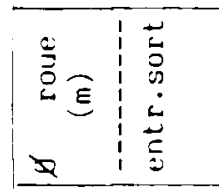 & $\stackrel{i}{i}$ & $\underset{N}{i}$ & $\begin{array}{l}n \\
\tilde{u} \\
i \\
\Xi \\
= \\
=\end{array}$ & & $\begin{array}{l}\because \\
\vdots \\
0 \\
\vdots \\
-\end{array}$ & \\
\hline$>$ 它 है & & & $\infty$ & & & \\
\hline 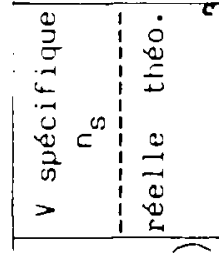 & & & $\begin{array}{l}\vec{b} \\
\vec{n} \\
\vec{n}\end{array}$ & & $\stackrel{\infty}{\Omega}$ & \\
\hline$>$ 完跣 & & $\because$ & 욤 & & $\hat{N}$ & $\stackrel{P}{\sim}$ \\
\hline$=\quad \frac{3}{2}$ & & & $\stackrel{m}{n}$ & & $\tilde{I}$ & \\
\hline$\therefore \quad \frac{\bar{n}}{m_{E}}$ & & $\bar{i}$ & \pm & & $\tilde{n}$ & \\
\hline 实离 & $\hat{N}$ & $\bar{N}$ & $\bar{m}$ & & $\cong$ & $\approx$ \\
\hline 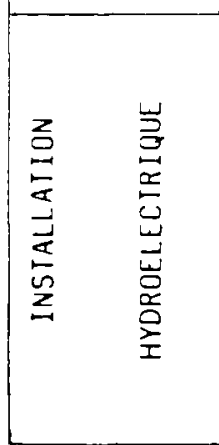 & 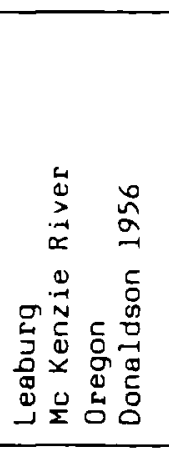 & 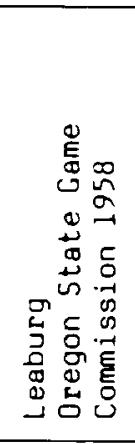 & 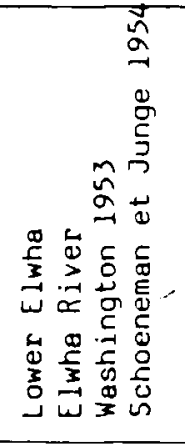 & 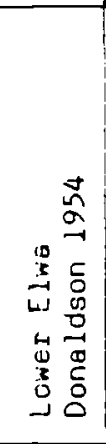 & 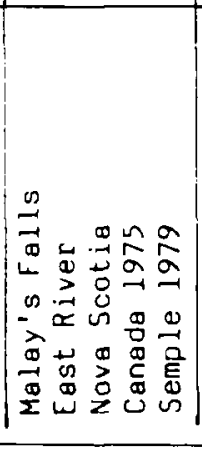 & 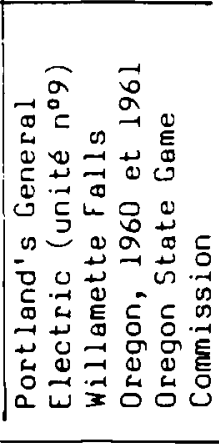 \\
\hline
\end{tabular}




\begin{tabular}{|c|c|c|c|c|c|}
\hline 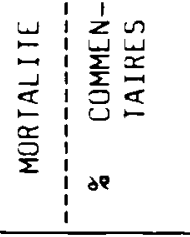 & 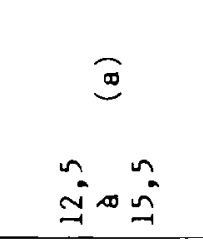 & 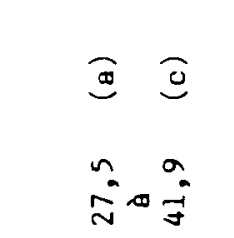 & 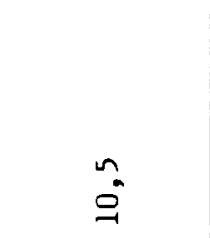 & $\underset{\sigma}{\approx}$ & 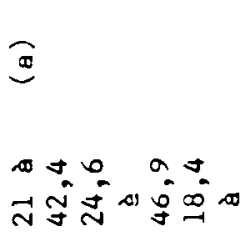 \\
\hline 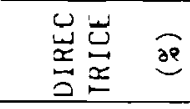 & $\stackrel{8}{9}$ & & 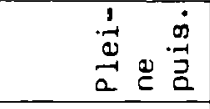 & 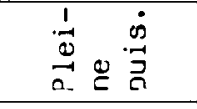 & $\exists \quad n$ \\
\hline 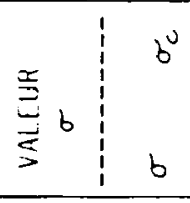 & & $\begin{array}{l}0 \\
0 \\
0 \\
\hat{\hat{N}} \\
0 \\
0 \\
0\end{array}$ & 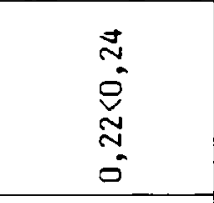 & 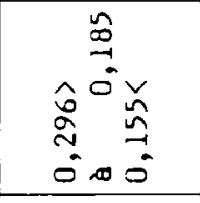 & $\begin{array}{l}\hat{x} \\
0 \\
0 \\
0 \\
0\end{array}$ \\
\hline$\stackrel{0}{=} \stackrel{D}{0}_{0}^{0}$ & tot & $\ddot{q}$ & + & i & $0^{-\infty} \neq$ \\
\hline 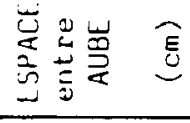 & & $\tilde{\sigma}$ & $\vec{m} \infty$ & $\cong$ & $\Xi$ \\
\hline 号亏岕 & & $\cong$ & $\exists$ & 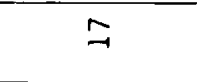 & $\leadsto$ \\
\hline 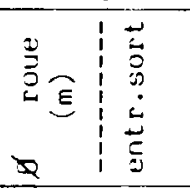 & $\bar{s}$ & $\begin{array}{l}0 \\
2 \\
- \\
0 \\
\vdots \\
\vdots\end{array}$ & $\begin{array}{l}\infty \\
a \\
m \\
\infty \\
m \\
m\end{array}$ & $\begin{array}{l}0 \\
0 \\
5 \\
0 \\
\vdots \\
m\end{array}$ & $\begin{array}{l}9 \\
\dot{s} \\
5\end{array}$ \\
\hline$>\stackrel{\dot{\omega}}{\Xi} \quad \widehat{n}$ & & $\vec{m}$ & $\stackrel{n}{n}$ & $\hat{\sim}$ & $\vec{m}$ \\
\hline 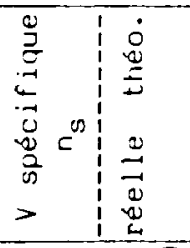 & & 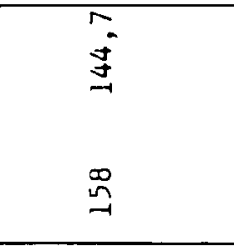 & $\begin{array}{l}\stackrel{n}{a} \\
\stackrel{N}{N} \\
\stackrel{\infty}{N}\end{array}$ & $\begin{array}{l}\overrightarrow{2} \\
\vec{n}\end{array}$ & $\begin{array}{l}\stackrel{\mathcal{J}}{\exists} \\
\stackrel{\mathcal{I}}{\exists}\end{array}$ \\
\hline$>\stackrel{\circ}{\approx} \quad \frac{\overparen{E}}{\omega}$ & $\stackrel{s}{\therefore}$ & $\stackrel{\Sigma}{\sim}$ & $\stackrel{\curvearrowright}{\underset{二}{(}}$ & 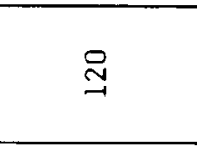 & $\stackrel{n}{\infty}$ \\
\hline$\widehat{\frac{x}{2}}$ & $\begin{array}{l}\vdots \\
\vdots\end{array}$ & i & $\tilde{m}$ & $\stackrel{0}{3}$ & $\approx$ \\
\hline$=m_{E}^{0}$ & $\because$ & $\vec{m}$ & $\cong$ & $\stackrel{\Im}{\Im}$ & $\tilde{8}$ \\
\hline 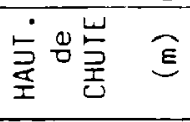 & $\stackrel{?}{\square}$ & m & $\tilde{n}$ & $\tilde{\sigma}$ & 응 \\
\hline 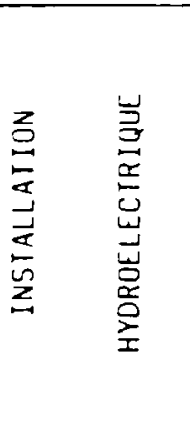 & 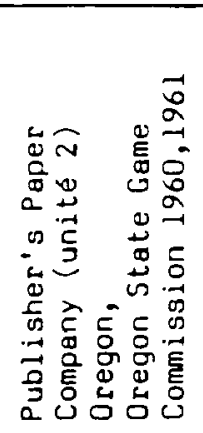 & 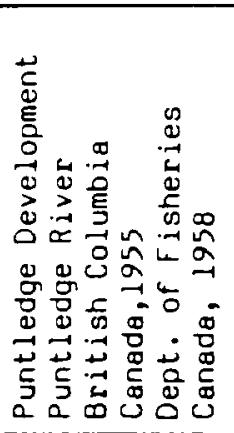 & 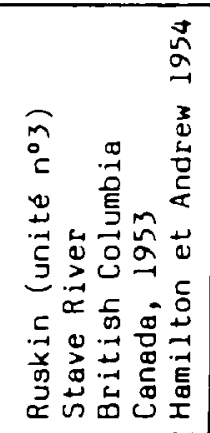 & 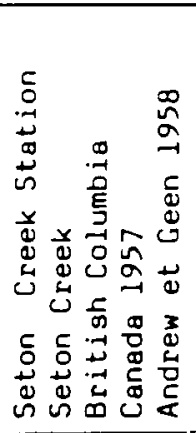 & 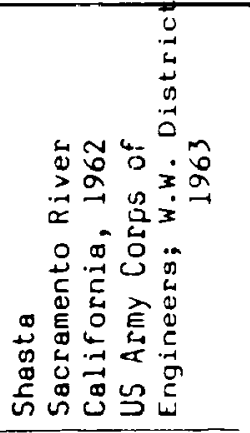 \\
\hline
\end{tabular}




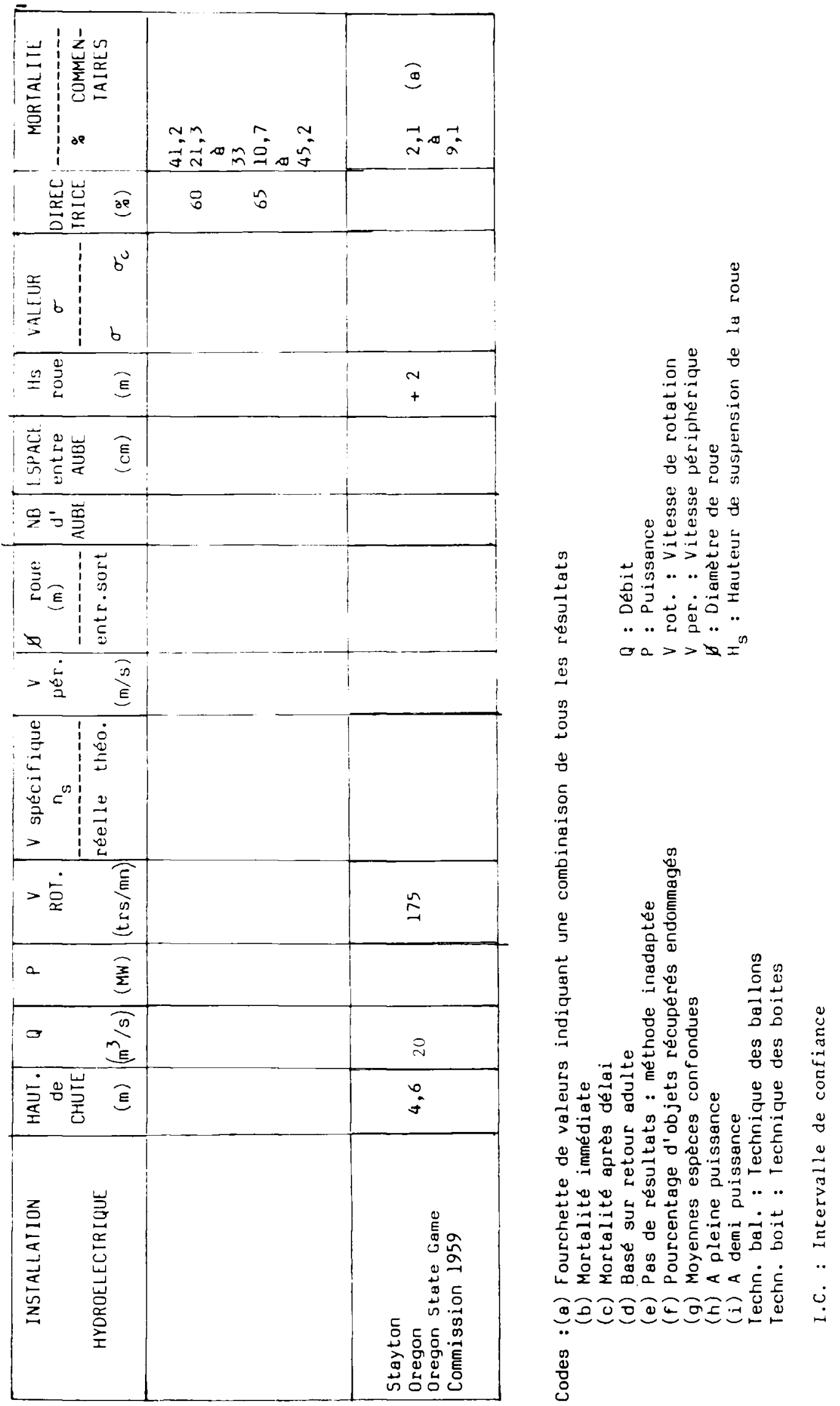




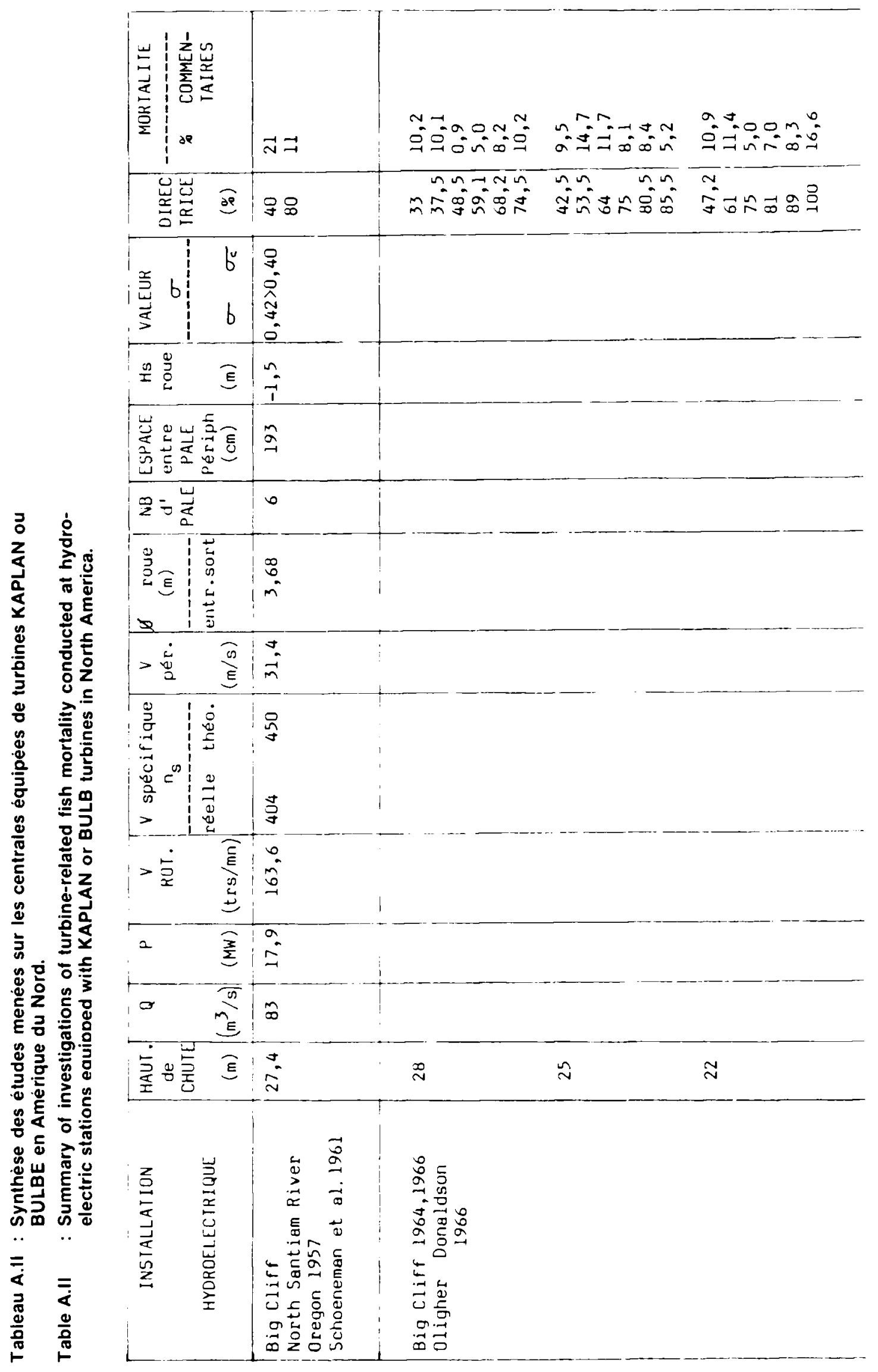




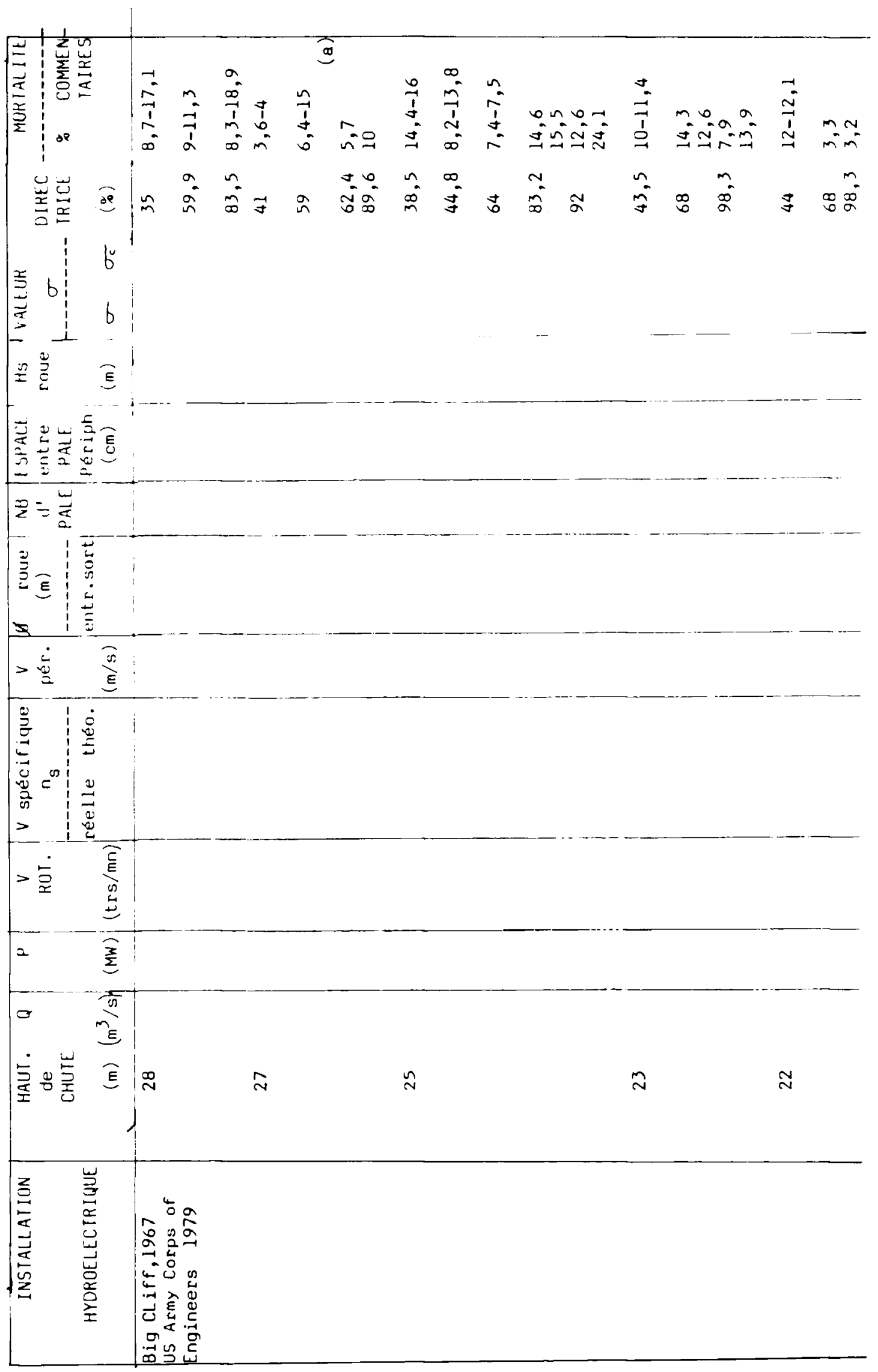




\begin{tabular}{|c|c|c|c|c|c|c|c|c|c|}
\hline 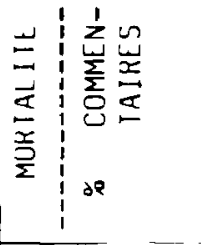 & $\begin{array}{l}3 \\
\because \\
=\end{array}$ & 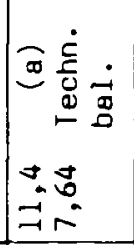 & $\underset{N}{2}$ & & 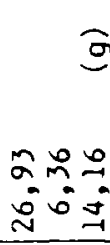 & $\begin{array}{l}0 \\
0 \\
=-1\end{array}$ & $m_{m i n}^{n}$ & $\cong \infty r^{\infty}$ & $\stackrel{\infty}{\infty}$ \\
\hline 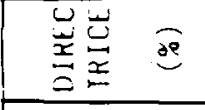 & & & & & 0 & $\approx$ & in & $\stackrel{n}{n}$ & $\stackrel{8}{\circ}$ \\
\hline 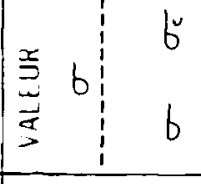 & $\begin{array}{l}n \\
n \\
0 \\
\hat{0} \\
\vdots \\
0\end{array}$ & & & $\vec{a}$ & $\infty$ & ñ & & & \\
\hline 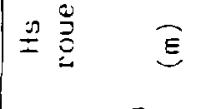 & 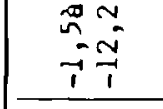 & & & & & & & & \\
\hline 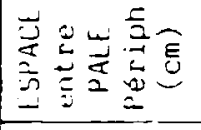 & F & & 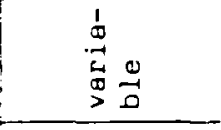 & $\stackrel{m}{\Rightarrow}$ & & & & & \\
\hline $\bar{z} \bar{z}=\underset{j}{\dot{z}}$ & un & & $m$ & 0 & & & & & \\
\hline 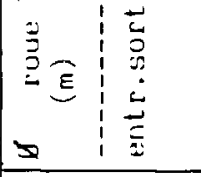 & $\because$ & & $\frac{i}{n}$ & $\tilde{n}$ & & & & & \\
\hline$>\dot{\dot{\omega}} \quad \stackrel{\Xi}{\Xi}$ & $\stackrel{D}{\sim}$ & & $\approx$ & $\vec{m}$ & & & & & \\
\hline 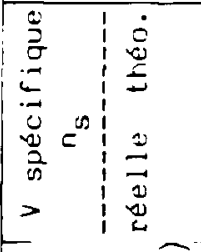 & $\begin{array}{l}\tilde{n} \\
\tilde{F} \\
\tilde{H}\end{array}$ & & & & & & & & \\
\hline$>\underset{\vec{x}}{\dot{\vec{E}}}$ & $\cong$ & & $\stackrel{\infty}{\infty}$ & $\hat{\imath}$ & & & & & \\
\hline $1 \quad \widehat{\frac{\pi}{2}}$ & 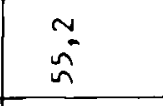 & & $\stackrel{D}{r}$ & $\stackrel{3}{0}$ & & & & & \\
\hline$=i_{\varepsilon}^{\infty}$ & 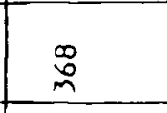 & & 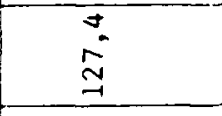 & & & & & & \\
\hline 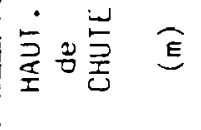 & $\tilde{\infty}$ & $\stackrel{\infty}{=}$ & $\begin{array}{l}\vec{\infty} \\
\infty\end{array}$ & ㅁำ & $\stackrel{n}{m} \tilde{m}$ & $\stackrel{n}{n} \tilde{m}$ & $\stackrel{\sim}{m} \tilde{m}$ & $\stackrel{n}{m} \stackrel{n}{m}$ & $\stackrel{\infty}{N}$ \\
\hline 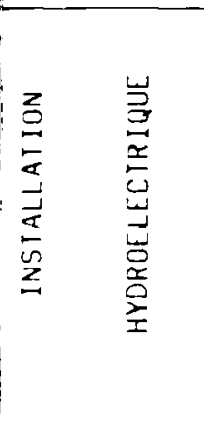 & 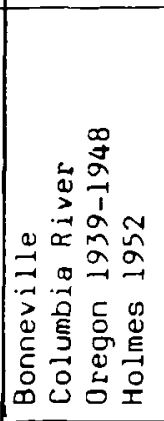 & 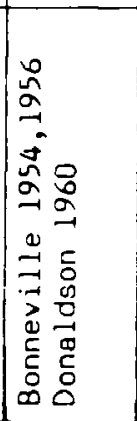 & 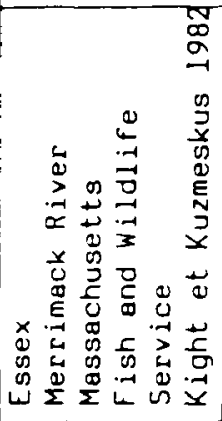 & 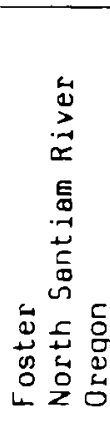 & 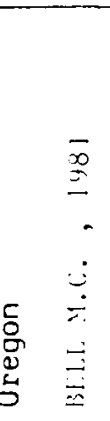 & & & & \\
\hline
\end{tabular}


Bull. Fr. Pêche Piscic. (1989) $\mathbf{3 1 2}-\mathbf{3 1 3}-62-$

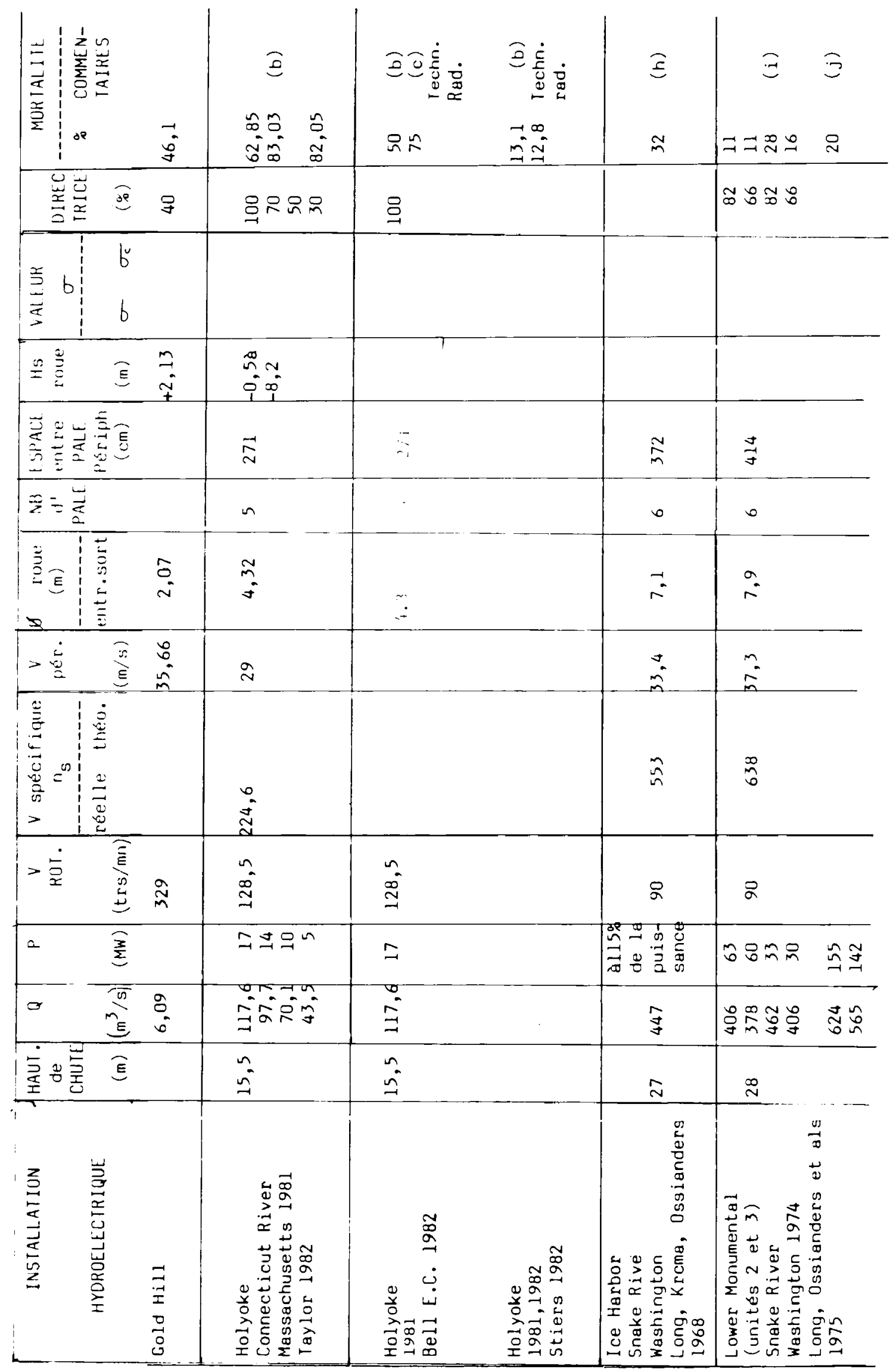




\begin{tabular}{|c|c|c|c|c|c|c|}
\hline 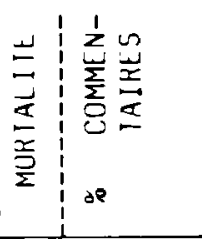 & 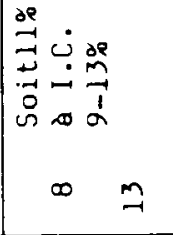 & 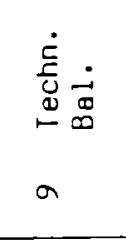 & 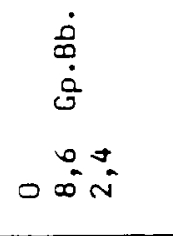 & $\begin{array}{l}2 \\
2=\end{array}$ & $\begin{array}{l}3 \\
=\end{array}$ & 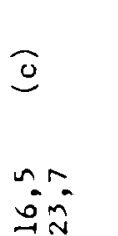 \\
\hline 岕岂 & $\stackrel{\supset}{\wedge}$ & & & & $\underline{x}$ & \\
\hline 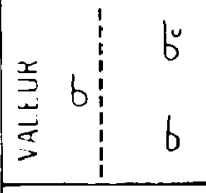 & $\begin{array}{l}0 \\
0 \\
\hat{n} \\
0 \\
0\end{array}$ & & & & $\begin{array}{l}\hat{0} 0 \\
\hat{N} \\
0 \\
0 \\
0\end{array}$ & \\
\hline 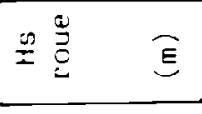 & $\stackrel{0}{i}$ & & $\stackrel{i}{i}$ & $\hat{i}$ & $\stackrel{\infty}{\infty}$ & \\
\hline 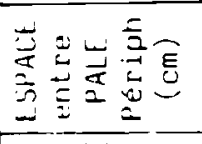 & $\approx$ & & $\underset{\sigma}{\vec{\sigma}}$ & & 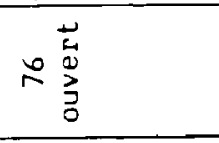 & \\
\hline$\frac{m}{2}=\frac{\breve{a}}{a}$ & 0 & & $n$ & & $n$ & \\
\hline 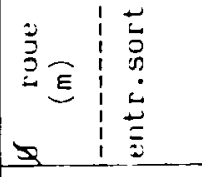 & $\exists$ & & 怘 & & $\begin{array}{l}\vec{b} \\
i\end{array}$ & \\
\hline$>$ है & $\tilde{m}$ & & $\begin{array}{l}\tilde{z} \\
\exists\end{array}$ & & $\vec{m}$ & \\
\hline 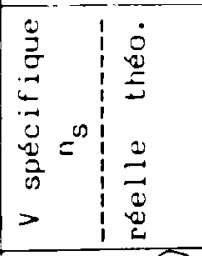 & $\begin{array}{l}\tilde{\sigma} \\
\tilde{\sigma}\end{array}$ & & $\tilde{\Xi}$ & & 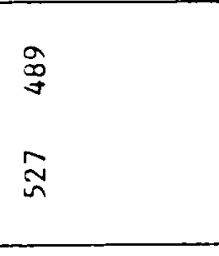 & \\
\hline 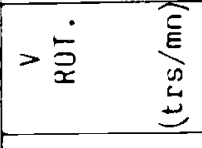 & $\infty$ & & 요 & 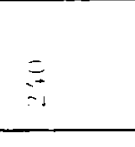 & $\hat{N}$ & \\
\hline 弪 & $\hat{\infty}$ & & $\hat{n}$ & $\because$ & $\stackrel{9}{\rho}$ & \\
\hline $0 \quad m_{E}^{\infty}$ & $\stackrel{p}{p}$ & & 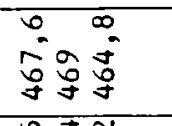 & $=r$ & $\vec{n}$ & \\
\hline 室号戛 & $\tilde{n}$ & & 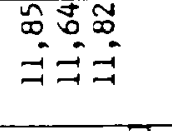 & 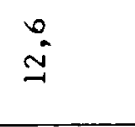 & $\hat{\sim}$ & \\
\hline 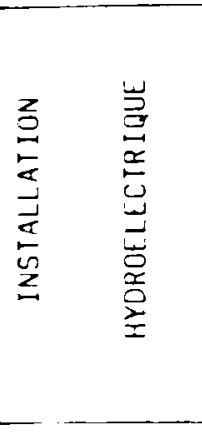 & 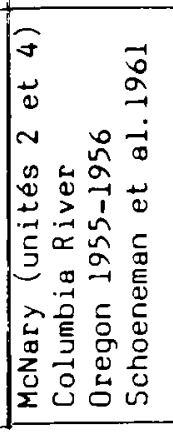 & 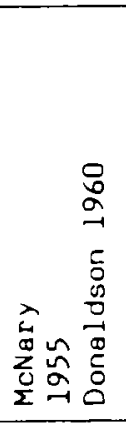 & 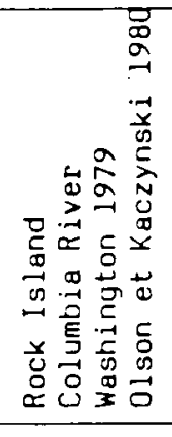 & 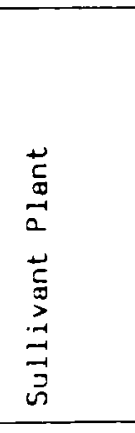 & 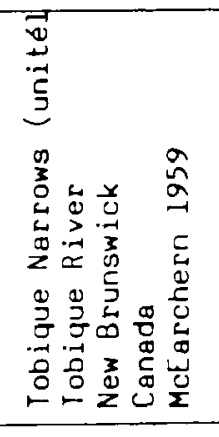 & 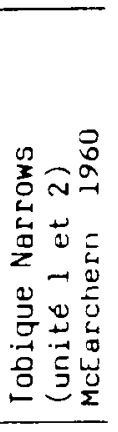 \\
\hline
\end{tabular}




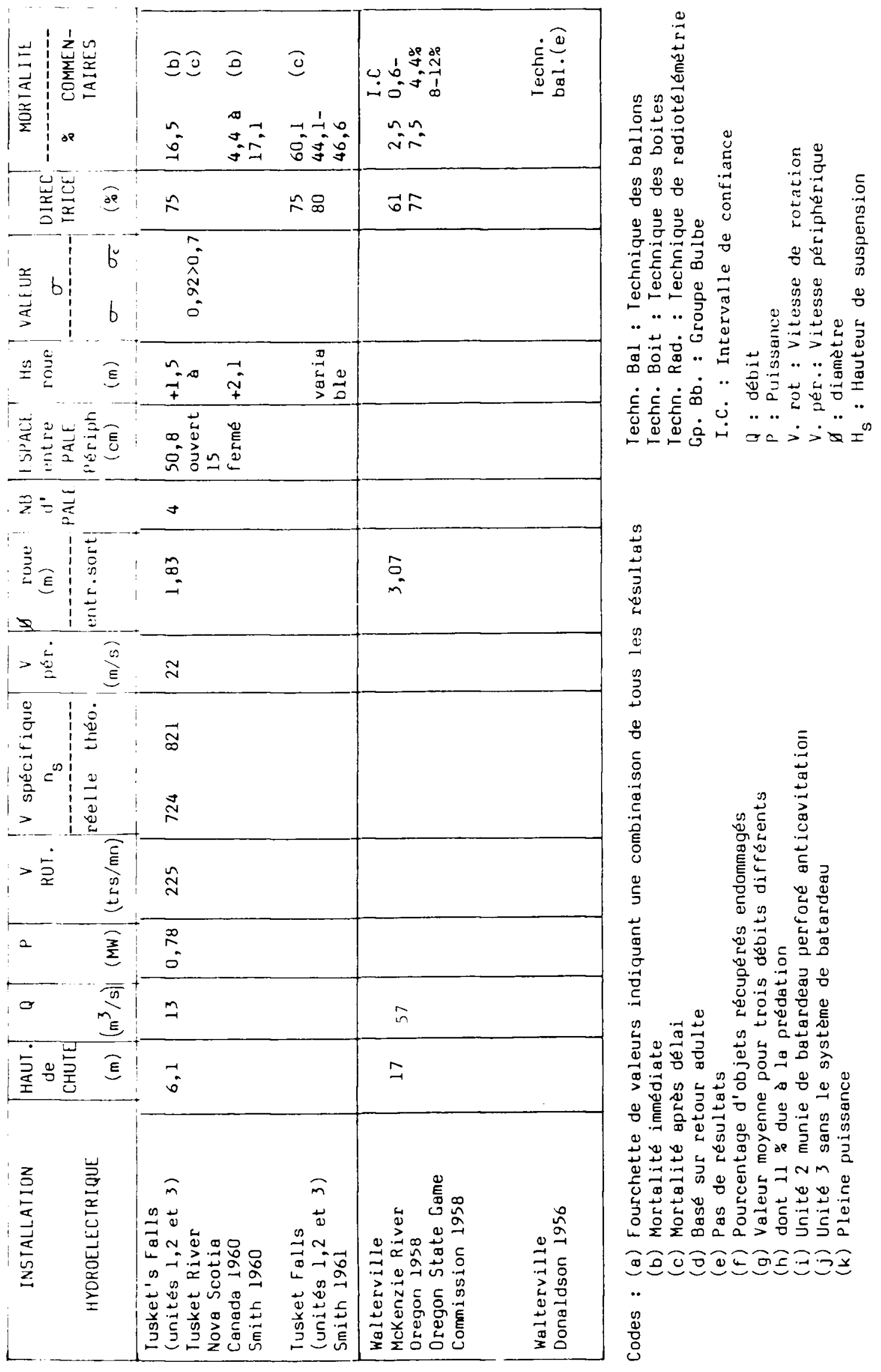




\begin{tabular}{|c|c|c|c|c|c|}
\hline & 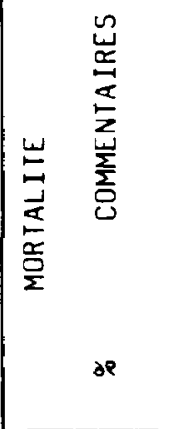 & 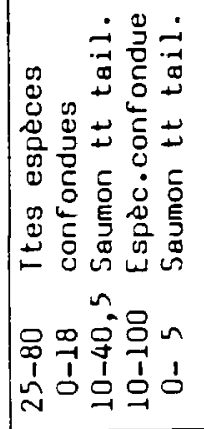 & 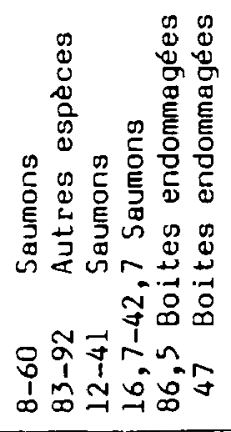 & 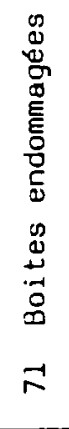 & 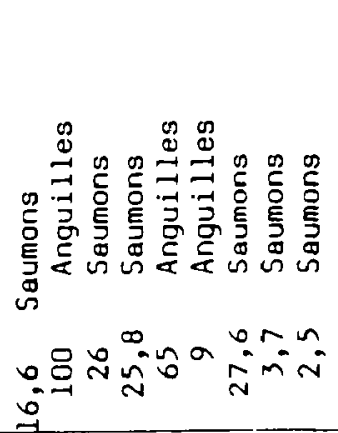 \\
\hline & de $\begin{array}{ll}\text { 总 } \\
\text { 岂 }\end{array}$ & \& $\quad \stackrel{0}{0}$ & 응응유 & & 号实 \\
\hline & 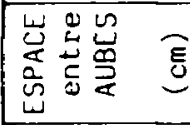 & 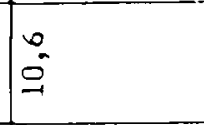 & & $\tilde{n}$ & \pm \\
\hline & 电方㟧 & 2 & & $\simeq$ & $\stackrel{\infty}{\rightarrow}$ \\
\hline 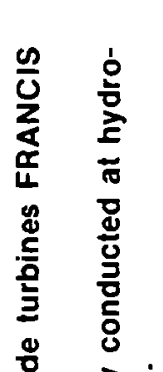 & 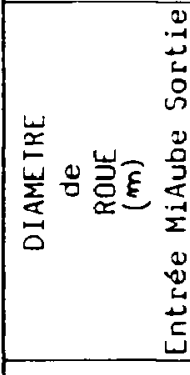 & 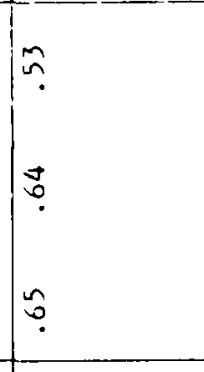 & & $\stackrel{2}{r}$ & $\begin{array}{l}\stackrel{\sim}{a} \\
\stackrel{n}{0} \\
\vdots\end{array}$ \\
\hline 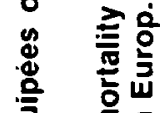 & 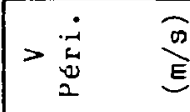 & $\hat{y=}=$ & $\ddot{m} \cong$ & & $m$ \\
\hline 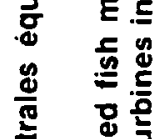 & $>\underset{\substack{n \\
\sim}}{n} e^{n}$ & 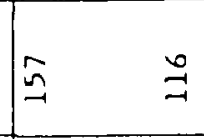 & & & 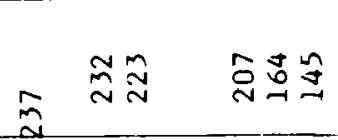 \\
\hline 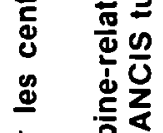 & $>\dot{0}$ & 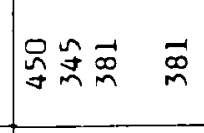 & aे & $\stackrel{R}{n}$ & 号 \\
\hline $\begin{array}{ll}5 & \frac{\pi}{5} \\
0 & \frac{\pi}{2} \\
0 & 0\end{array}$ & $a$ & $\stackrel{0}{0} \quad \stackrel{0}{\circ}$ & 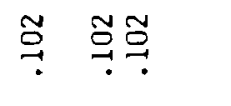 & & 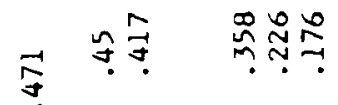 \\
\hline 造递 & $=m_{E}^{\sigma}$ & $\vec{\infty} \quad \stackrel{0}{?}$ & $\vec{\infty} \quad \overrightarrow{0} \overrightarrow{0} \stackrel{0}{\stackrel{0}{\longrightarrow}}$ & 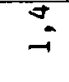 & o mà ññ \\
\hline 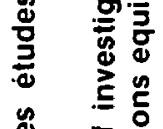 & 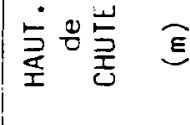 & $\begin{array}{l}0 \\
\Xi \\
\Xi\end{array}$ & & $\stackrel{\infty}{\circ}$ & $\stackrel{0}{\sim}$ \\
\hline 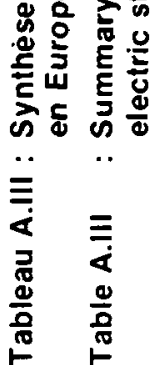 & 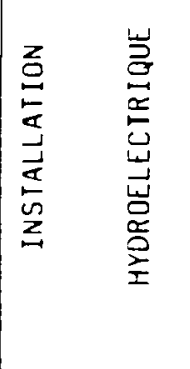 & 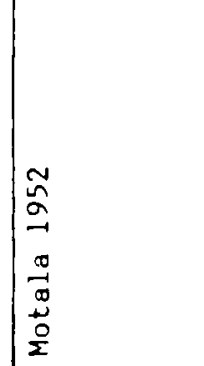 & 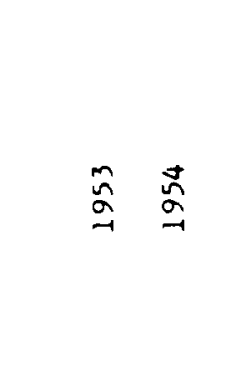 & 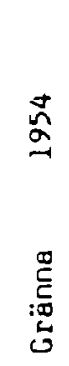 & 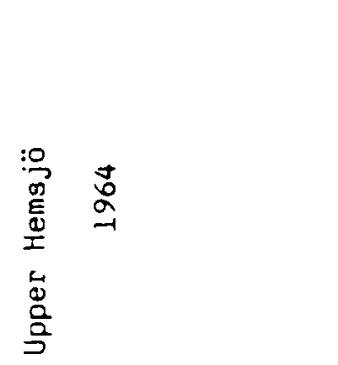 \\
\hline
\end{tabular}




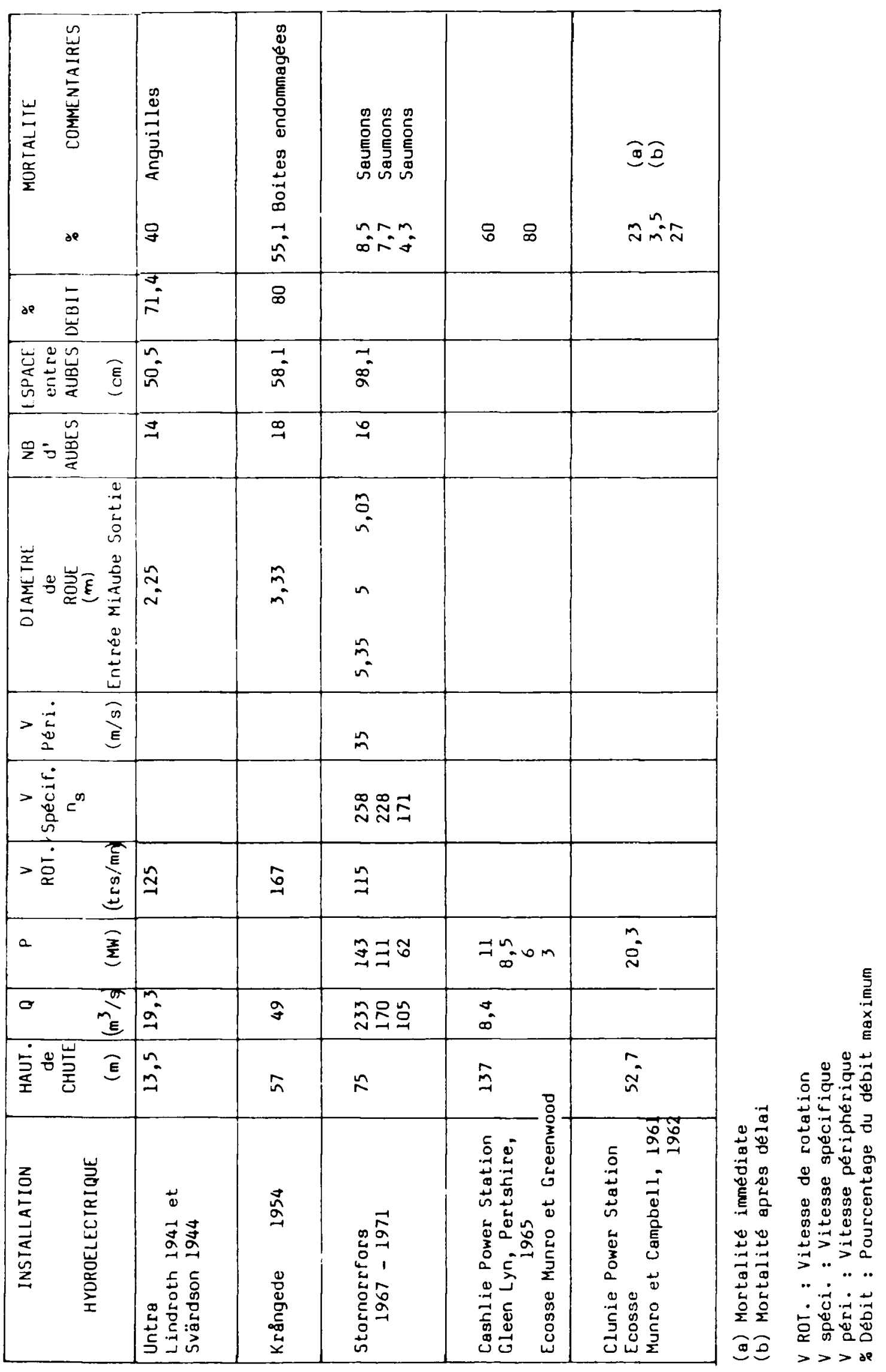




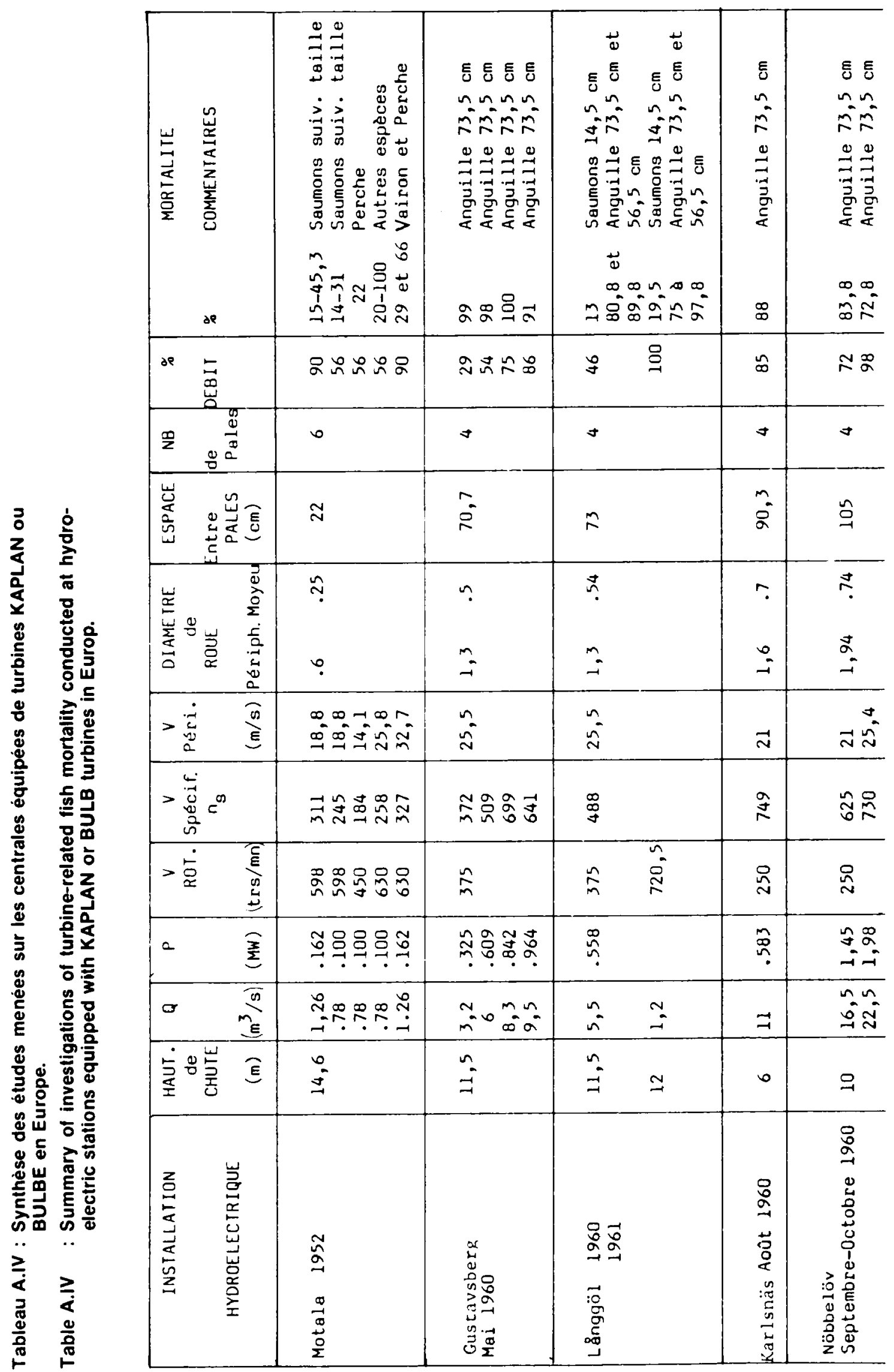




\begin{tabular}{|c|c|c|c|c|c|c|c|}
\hline 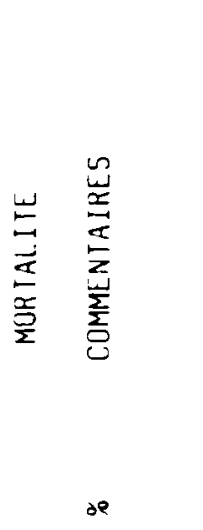 & 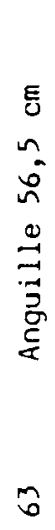 & 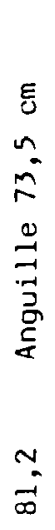 & 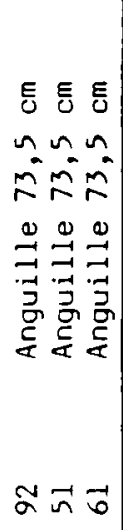 & 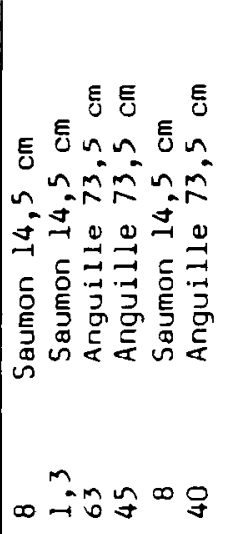 & $\begin{array}{c}0 \\
000 \\
0\end{array}$ & 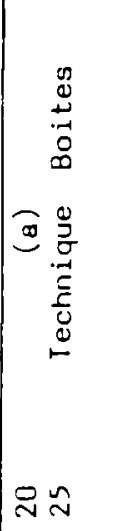 & 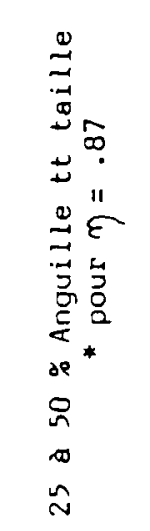 \\
\hline de $\stackrel{\square}{\stackrel{\Xi}{\Xi}}$ & $n$ & $g$ & $ㅇ ㅛ ㅇ ㅇ ㅛ$ & 吕 & & & 品只 \\
\hline$\frac{\infty}{2} \quad \frac{0}{2}$ & $\Delta$ & $\vec{\nabla}$ & $\checkmark$ & o & & & n \\
\hline 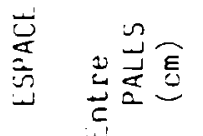 & $\exists$ & $\stackrel{m}{\exists}$ & $\stackrel{n}{0}$ & $\frac{a}{2}$ & & & I \\
\hline 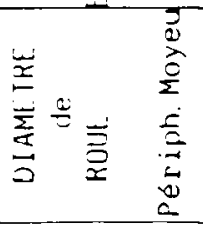 & $\tilde{\alpha}$ & $\begin{array}{l}\frac{\pi}{0} \\
0 \\
0 \\
0 \\
i\end{array}$ & $\stackrel{\sim}{i}$ & $\begin{array}{l}\infty \\
i \\
m \\
m\end{array}$ & & & $\begin{array}{l}\infty \\
\vdots \\
\approx \\
\approx \\
\vdots\end{array}$ \\
\hline$>\underset{i}{\dot{n}} \quad \stackrel{0}{0}$ & $a$ & $\hat{\sim}$ & $\stackrel{0}{i}$ & $\stackrel{0}{i}$ & & & $\stackrel{m}{m}$ \\
\hline$>\underbrace{\infty}_{\frac{\omega}{2}} c^{\infty}$ & a & 周 & $\hat{స ్}$ & 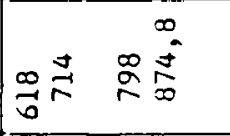 & & & $\stackrel{*}{\stackrel{*}{N}}$ \\
\hline 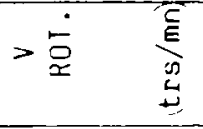 & $\underset{\infty}{\infty}$ & 怘 & $\stackrel{\text { 号 }}{\rightarrow}$ & $\cong$ & & & $\stackrel{\square}{\infty}$ \\
\hline$\widehat{\frac{x}{2}}$ & స్ర్ర & $\stackrel{n}{m}$ & $\begin{array}{l}\text { g tom } \\
\text { àn } \\
\text { inm }\end{array}$ & $\underset{0}{\exists} \cong$ & $\cong \approx \approx n$ & $n g$ & $\begin{array}{l}\text { Fo } \\
0 \\
0 \\
0 \\
m\end{array}$ \\
\hline $0 \quad m_{E}^{n}$ & $\cong$ & 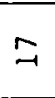 & 욤요 & $\operatorname{m}^{\circ}$ in & & & 品昌 \\
\hline 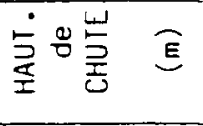 & 0 & $a$ & $n$ & 6 & พับลั & $\stackrel{a}{\approx}$ & m \\
\hline 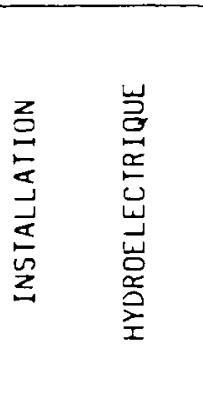 & 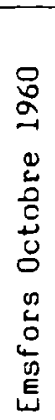 & 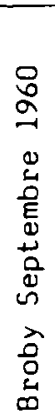 & 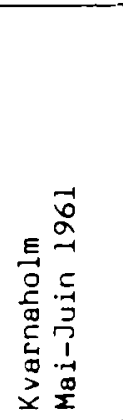 & 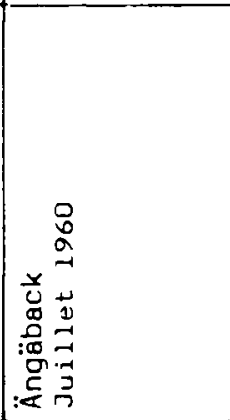 & 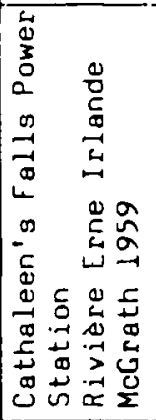 & 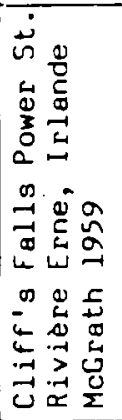 & 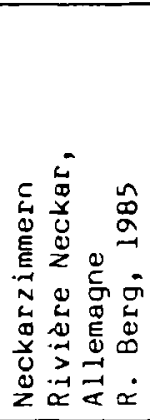 \\
\hline
\end{tabular}




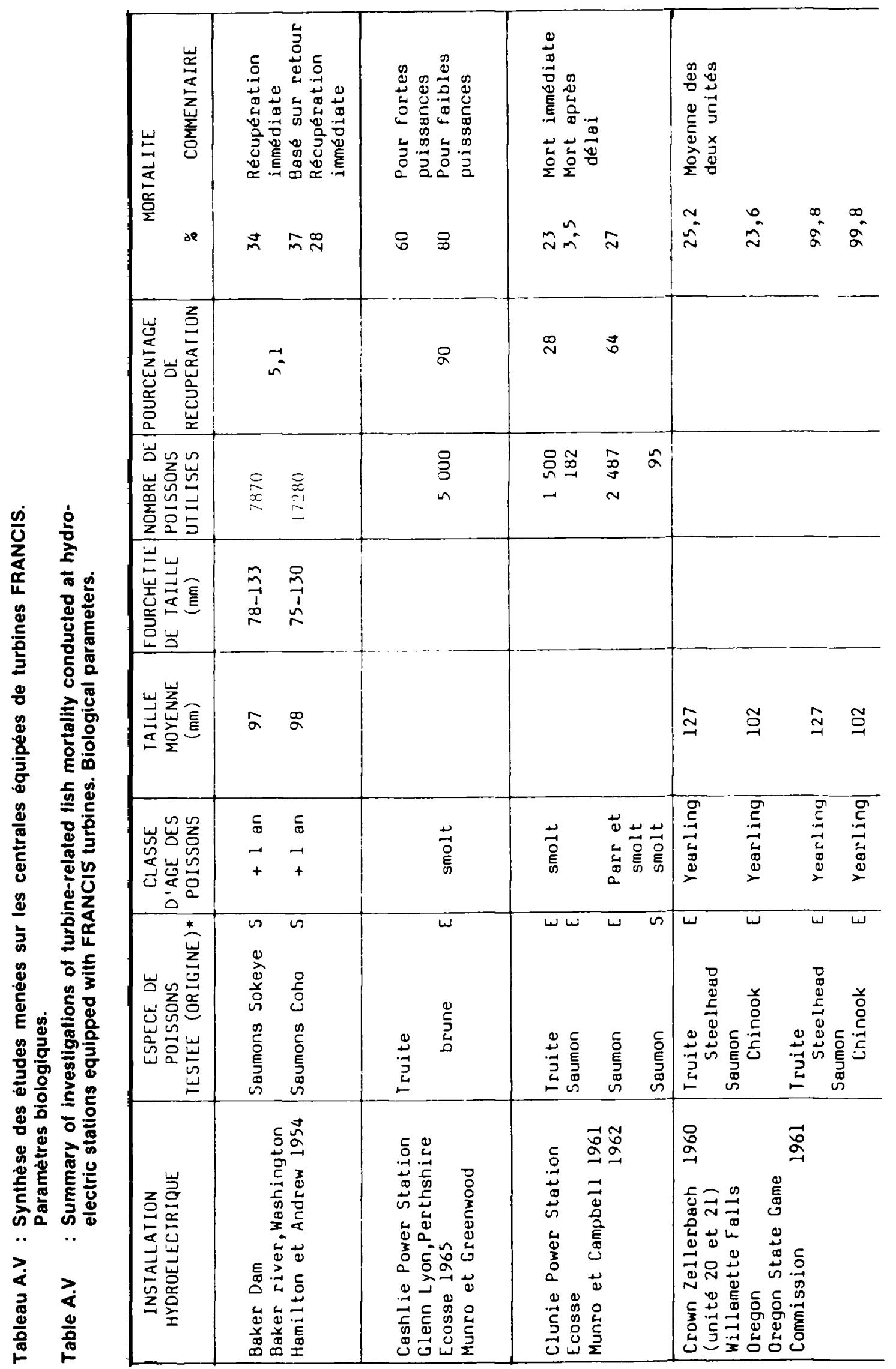




\begin{tabular}{|c|c|c|c|c|c|c|c|}
\hline 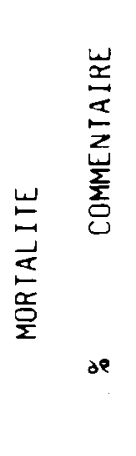 & 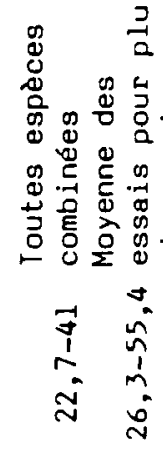 & 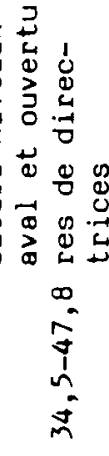 & 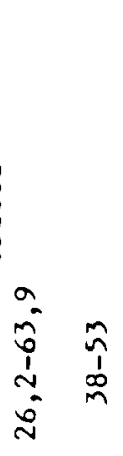 & $m \stackrel{m}{m}$ & 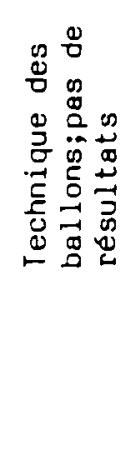 & 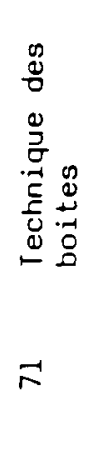 & $\hat{\xi}$ \\
\hline 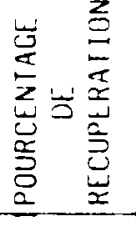 & ฉ & & $\alpha \approx$ & & & $\stackrel{\nexists}{\sim}$ & \\
\hline 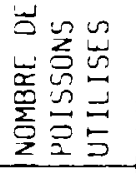 & $\begin{array}{l}\stackrel{\infty}{O} \\
\stackrel{\sim}{\sim}\end{array}$ & & \begin{tabular}{l} 
a \\
\multirow{\alpha}{N}{} \\
$\sim$
\end{tabular} & & & $\approx$ & \\
\hline 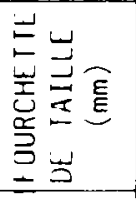 & $\begin{array}{ll}\tilde{b} & 0 \\
1 & 1 \\
7 & n\end{array}$ & 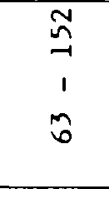 & 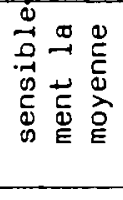 & 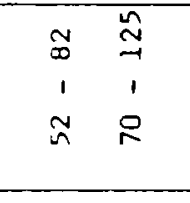 & & & \\
\hline 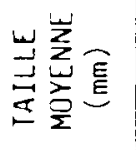 & $\hat{n}$ a & $\stackrel{ત}{\sim}$ & $\stackrel{0}{\sim}$ กี & $\stackrel{0}{\therefore}$ & & ఏ్త & \\
\hline 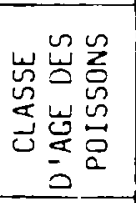 & 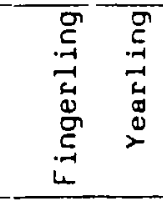 & 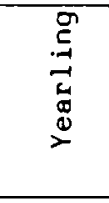 & 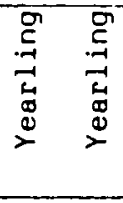 & 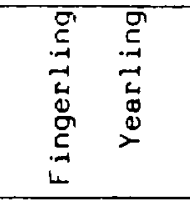 & & $\begin{array}{l}\text { ब } \\
-1 \\
+\end{array}$ & \\
\hline 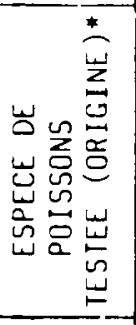 & 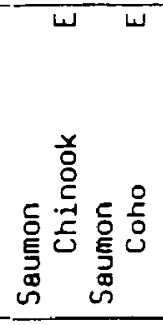 & 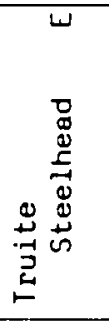 & 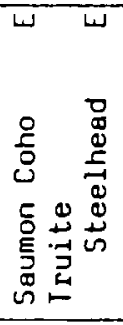 & 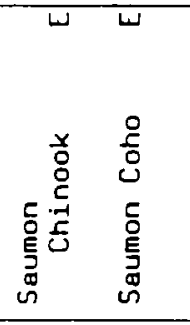 & & 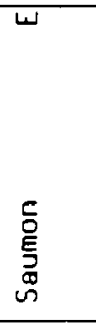 & 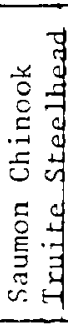 \\
\hline 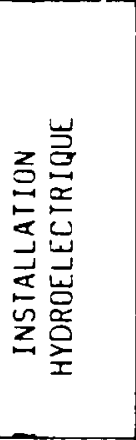 & 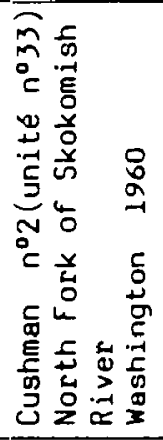 & 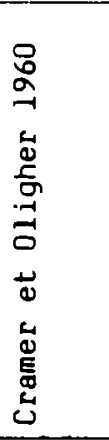 & $\begin{array}{l}\overrightarrow{0} \\
\stackrel{-}{-1}\end{array}$ & 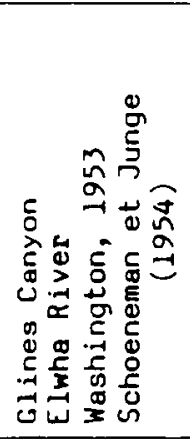 & 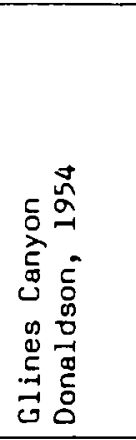 & 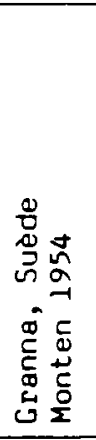 & $\begin{array}{l}\text { बे } \\
\approx \\
0 \\
0 \\
0 \\
\end{array}$ \\
\hline
\end{tabular}




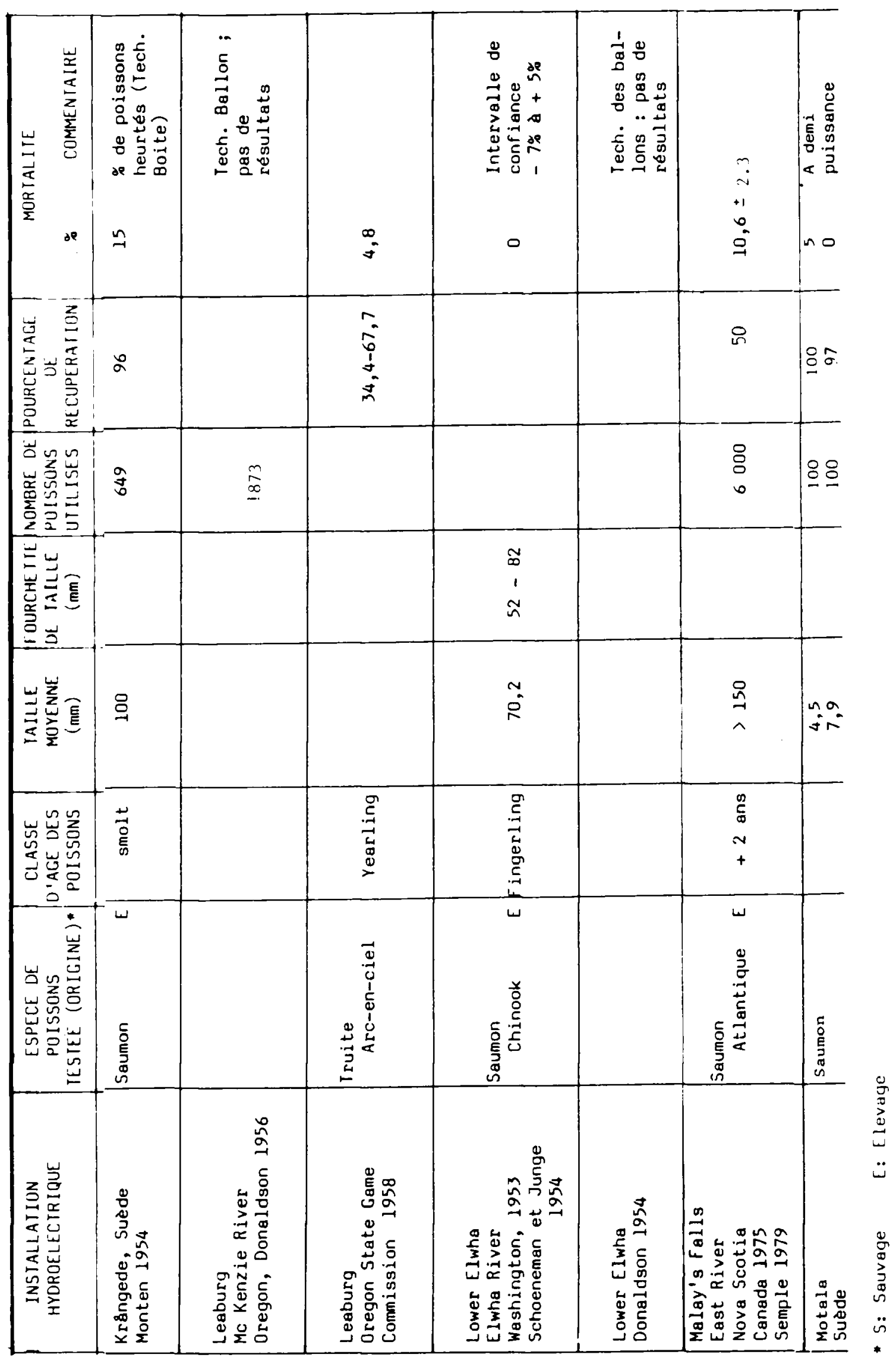




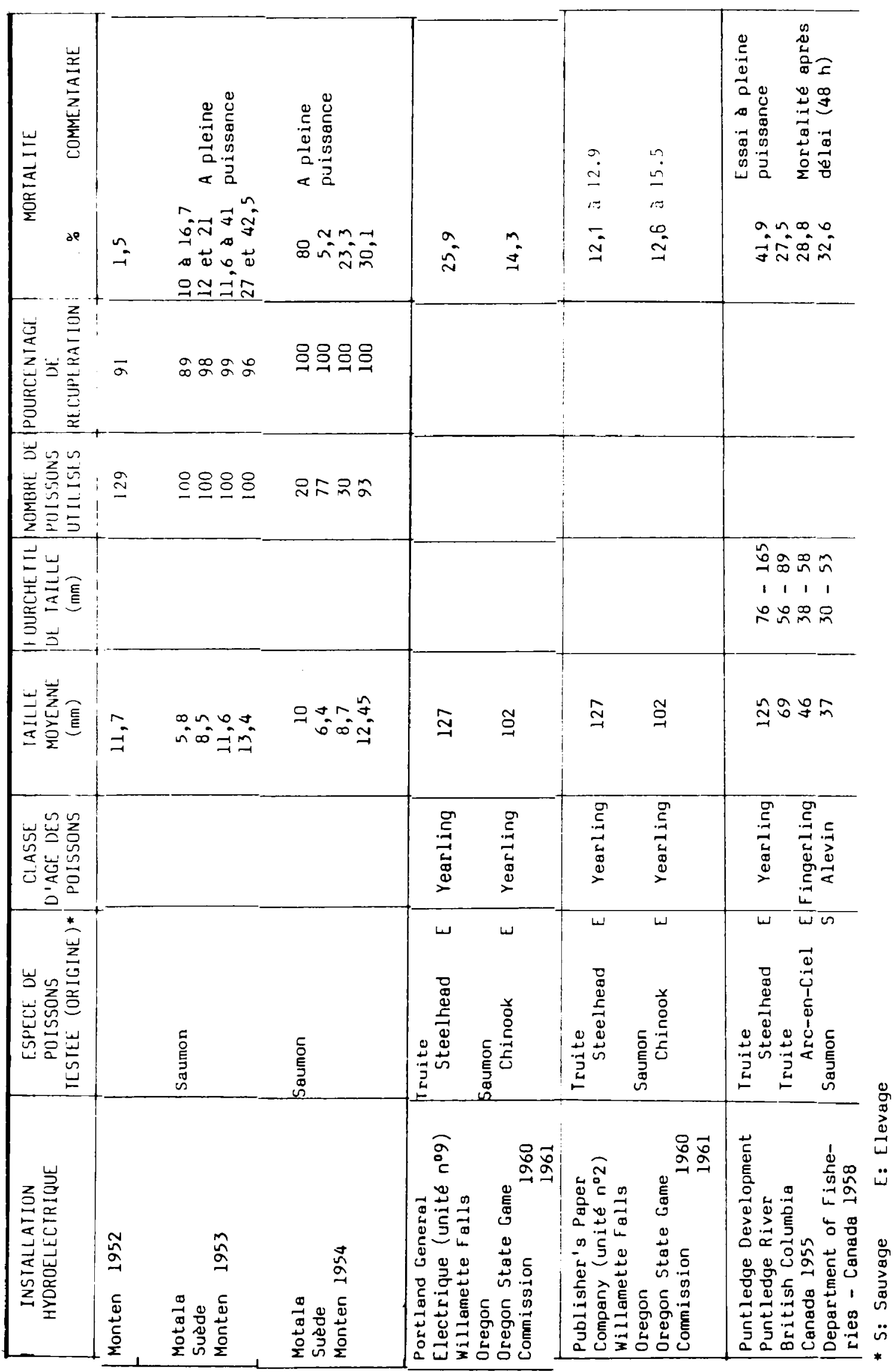




\begin{tabular}{|c|c|c|c|c|c|c|c|}
\hline 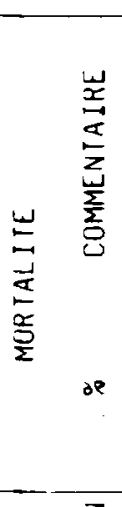 & 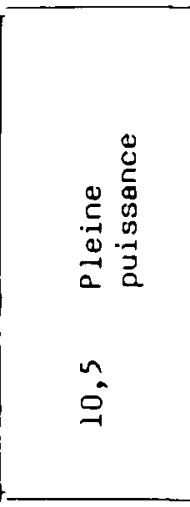 & 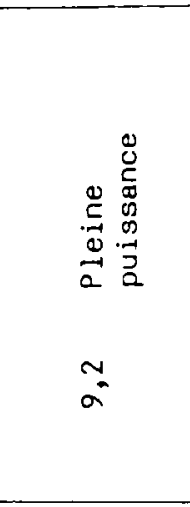 & 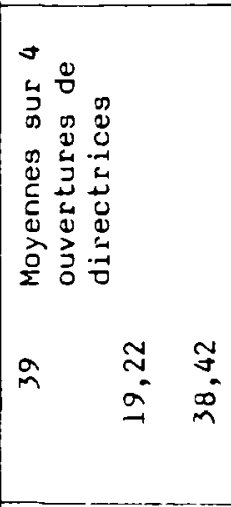 & 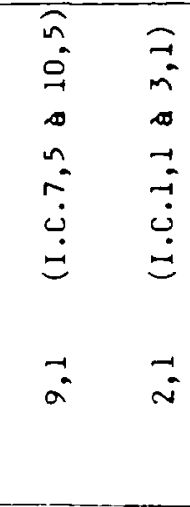 & 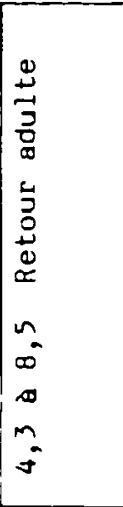 & 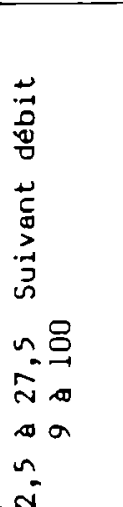 & F \\
\hline 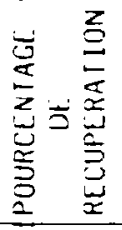 & $\sim$ & $\begin{array}{l}n \\
n \\
1 \\
0 \\
\infty\end{array}$ & $\approx \Re$ & 20 & $\dot{m}$ & ${ }_{a}^{a}$ & \\
\hline 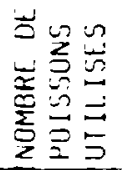 & 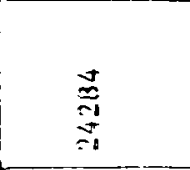 & $\begin{array}{l}\tilde{\infty} \\
\stackrel{\infty}{+} \\
0\end{array}$ & 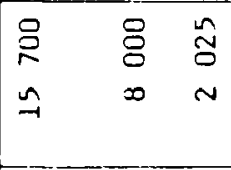 & & $\begin{array}{l}8 \\
8 \\
\sim \\
n\end{array}$ & $\check{\alpha}^{\infty}$ & \\
\hline 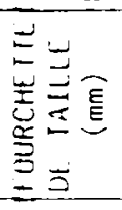 & 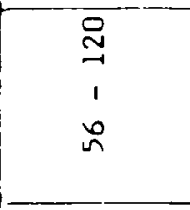 & $\begin{array}{l}a \\
1 \\
2\end{array}$ & & & & 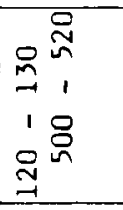 & $\begin{array}{l}8 \\
8 \\
1 \\
8 \\
0\end{array}$ \\
\hline 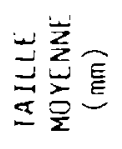 & $\infty$ & $\infty$ & $\stackrel{\sim}{\sim} \underset{\sim}{\sim}$ & & $\tilde{N}$ & 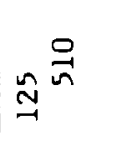 & \\
\hline 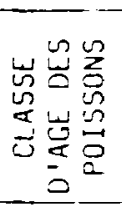 & 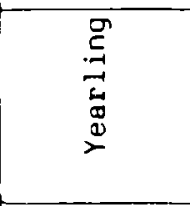 & 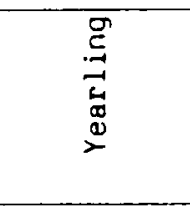 & 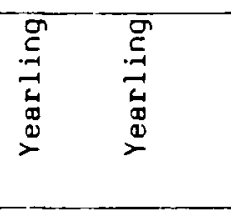 & 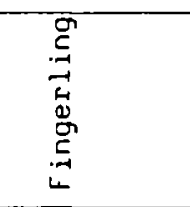 & $\begin{array}{l}m \\
\stackrel{9}{\sigma} \\
N \\
+ \\
+\end{array}$ & & \\
\hline 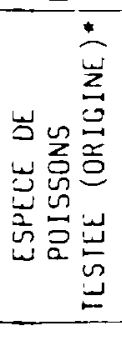 & 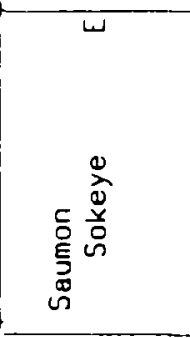 & 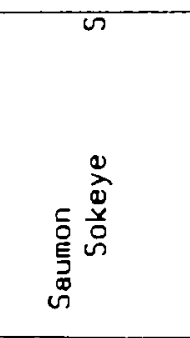 & 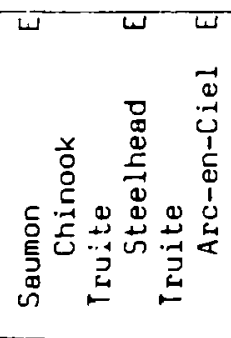 & एव & 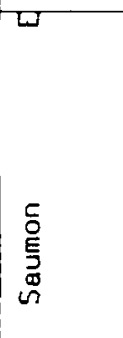 & 章 & 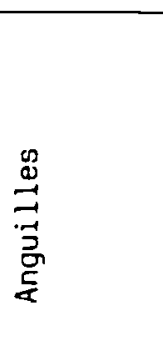 \\
\hline 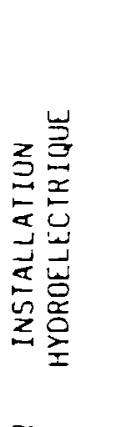 & 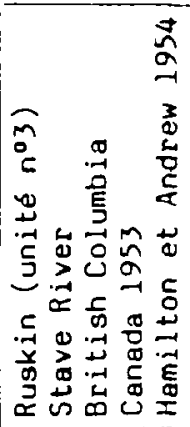 & 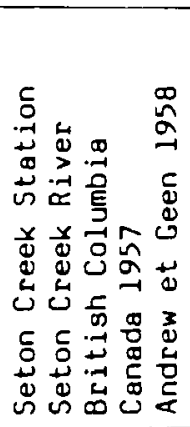 & 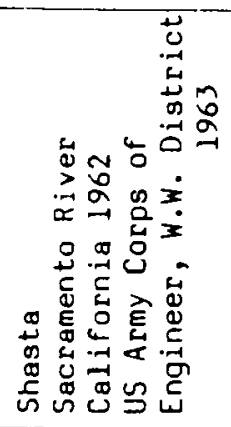 & 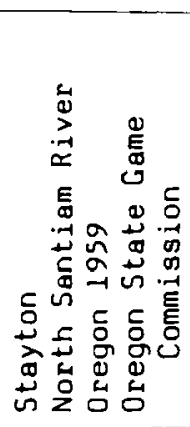 & 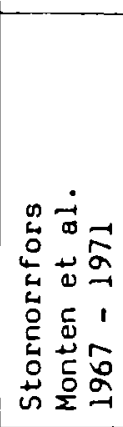 & 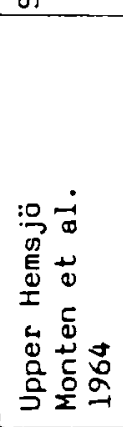 & 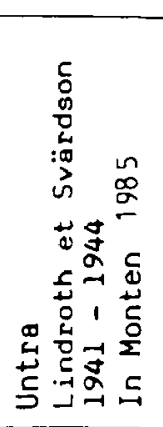 \\
\hline
\end{tabular}




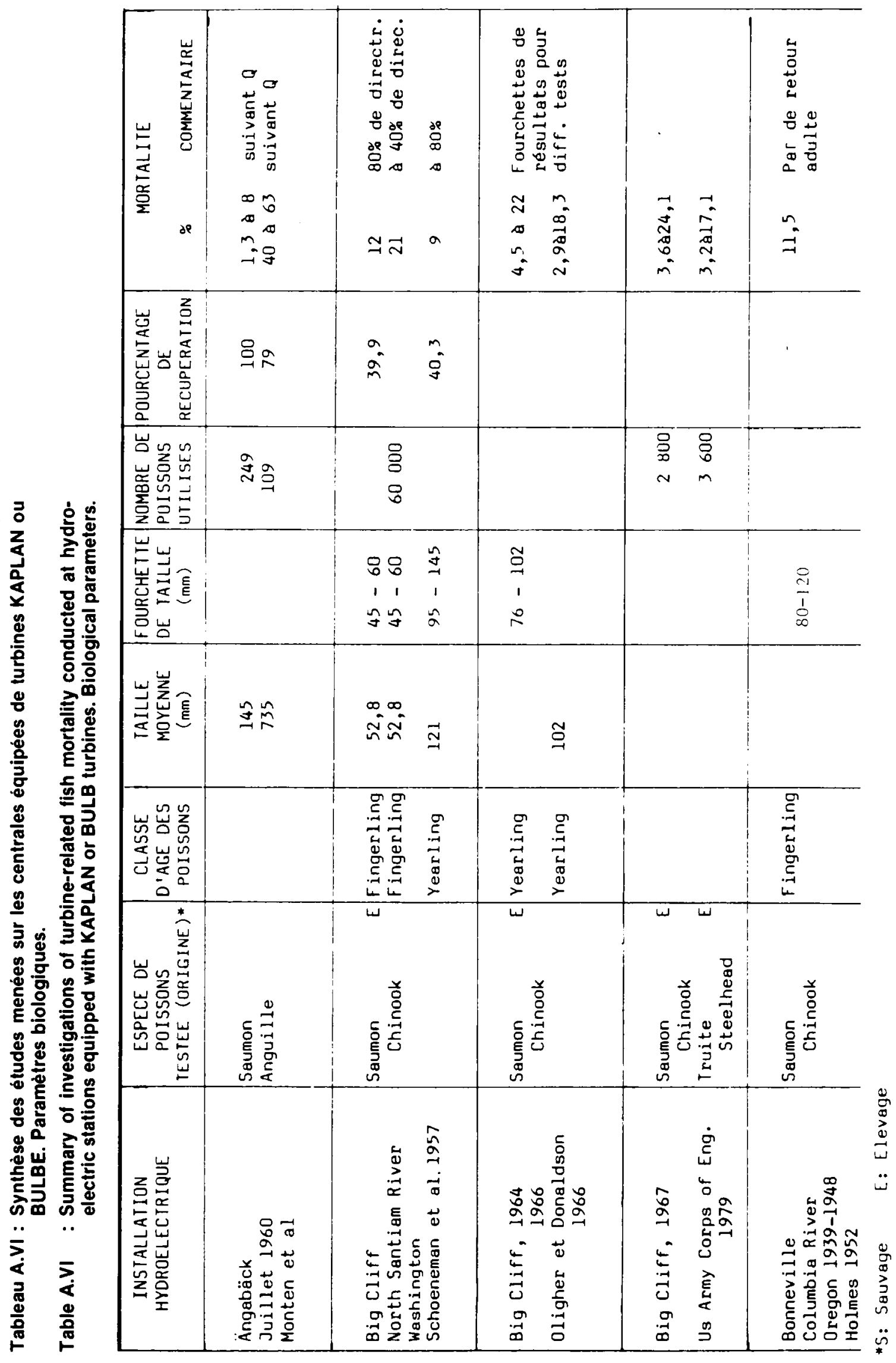




\begin{tabular}{|c|c|c|c|c|c|c|}
\hline 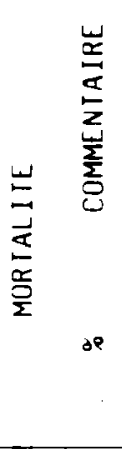 & 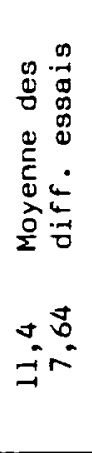 & 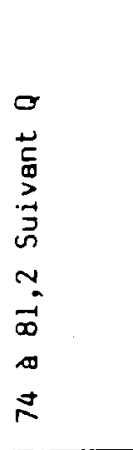 & 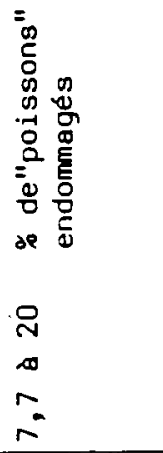 & 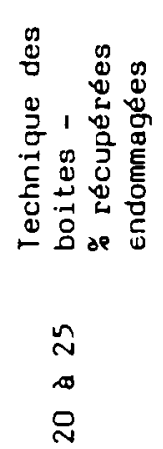 & $\begin{array}{l}05 \\
3 e \\
80 \\
80 \\
6 \%\end{array}$ & 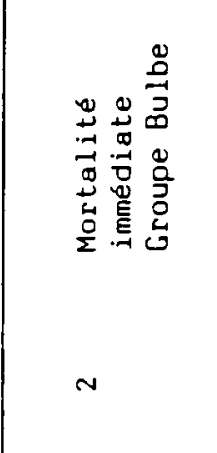 \\
\hline 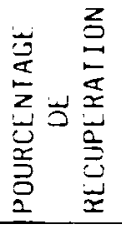 & थें & $\hat{\infty}$ & & & $\hat{o}$ oี & 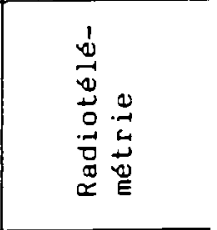 \\
\hline 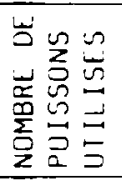 & 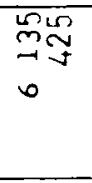 & $\stackrel{\infty}{\infty}$ & & $\infty$ & 㡶 & $\stackrel{8}{\circ}$ \\
\hline 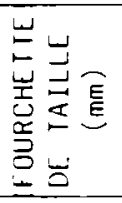 & & & & & & $\begin{array}{l}\text { 品 } \\
1 \\
\tilde{N}\end{array}$ \\
\hline 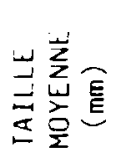 & & $\hat{N}$ & & & $\stackrel{n}{n} \tilde{n}$ & \\
\hline 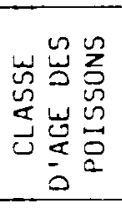 & 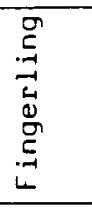 & & $\begin{array}{l}\stackrel{艹}{0} \\
\stackrel{\Xi}{E}\end{array}$ & $\begin{array}{l}\stackrel{\Delta}{0} \\
\stackrel{0}{E}\end{array}$ & & $\begin{array}{l}\stackrel{O}{E} \\
\stackrel{D}{N} \\
+\end{array}$ \\
\hline 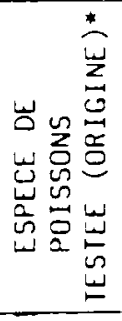 & 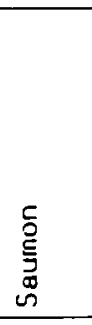 & 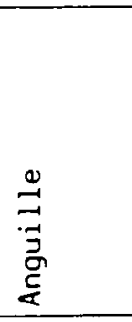 & 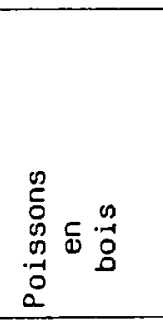 & 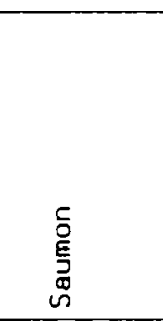 & 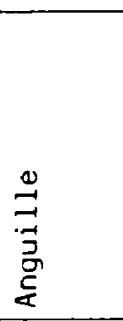 & 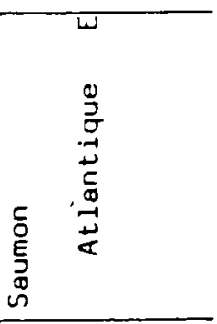 \\
\hline 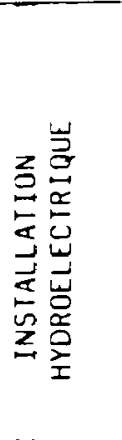 & 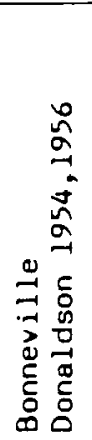 & 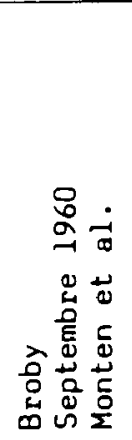 & 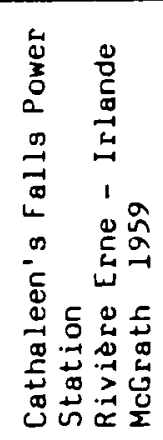 & 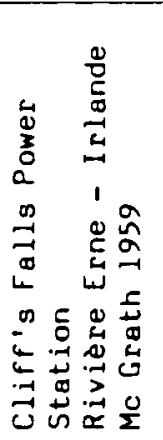 & 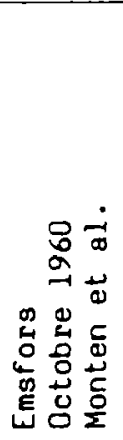 & 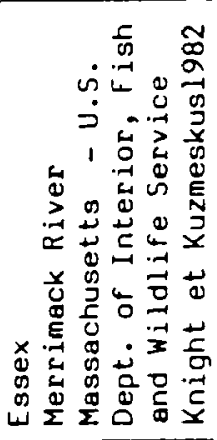 \\
\hline
\end{tabular}




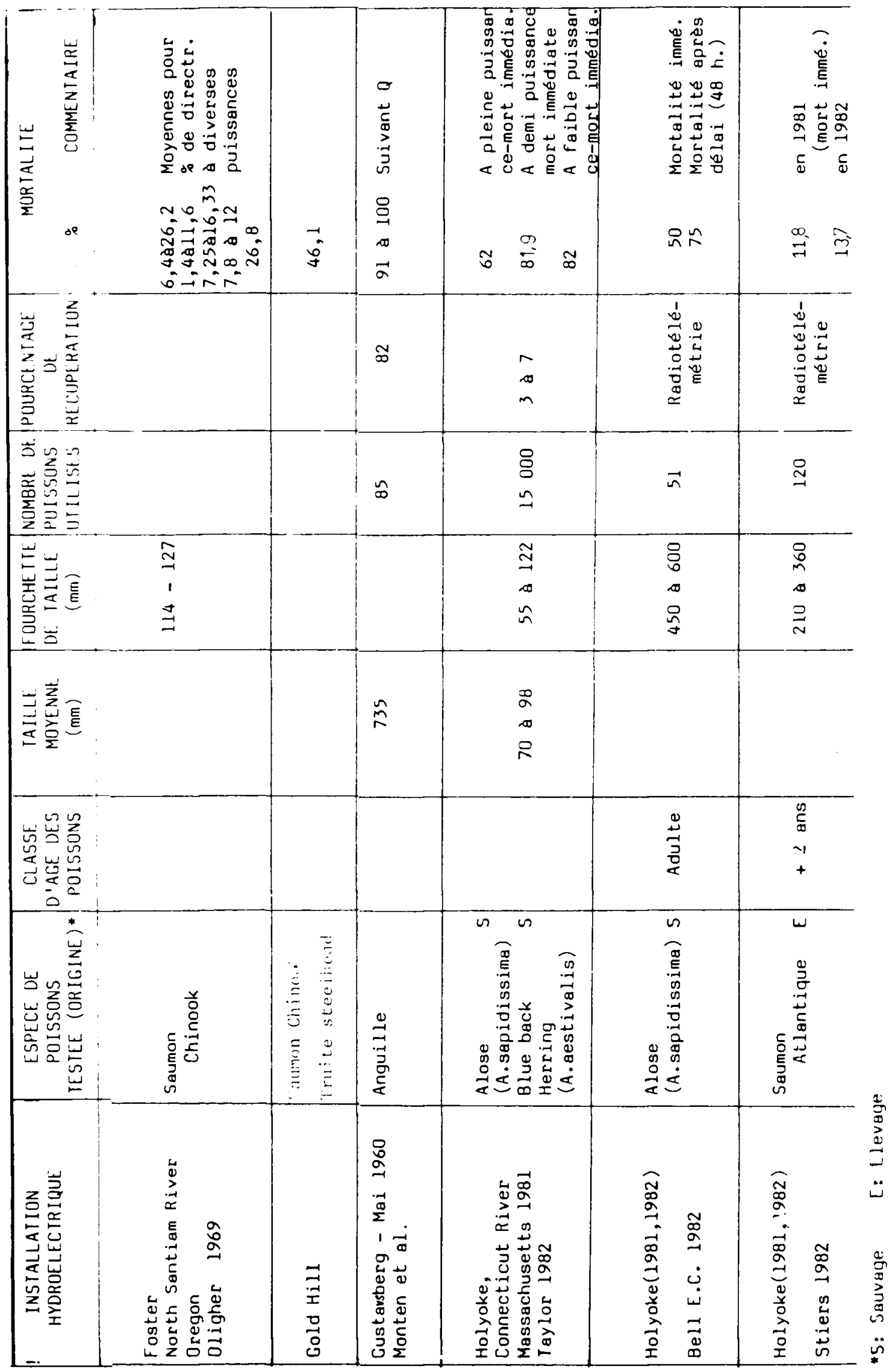




\begin{tabular}{|c|c|c|c|c|c|}
\hline 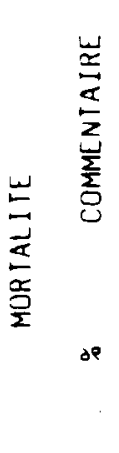 & 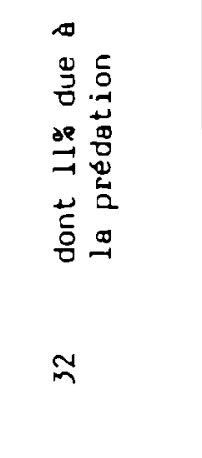 & 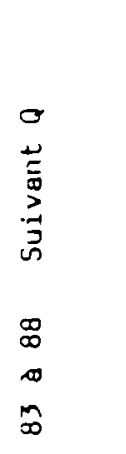 & 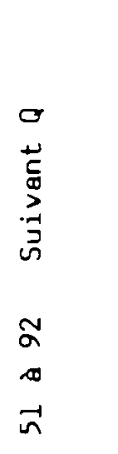 & 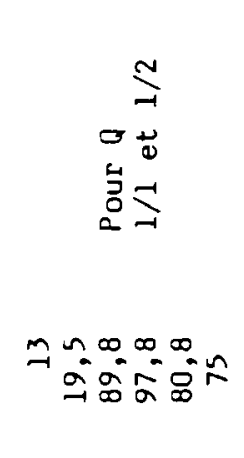 & 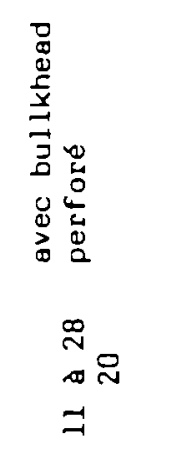 \\
\hline 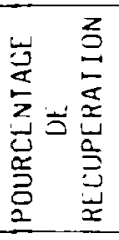 & $\exists$ & $\hat{b}$ & 2 & 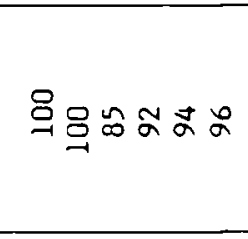 & $\begin{array}{l}0 \\
0 \\
0\end{array}$ \\
\hline 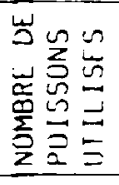 & $\begin{array}{l}\tilde{\hat{N}} \\
\vec{D} \\
\stackrel{a}{N}\end{array}$ & $\approx$ & 2 & 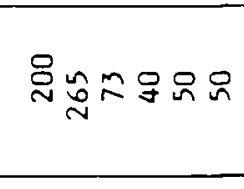 & $\begin{array}{l}\hat{0} \\
\hat{o} \\
\hat{o}\end{array}$ \\
\hline 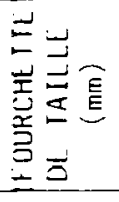 & & & & & \begin{tabular}{l}
9 \\
\hdashline \\
$\infty$ \\
0 \\
0
\end{tabular} \\
\hline 芝芳 & & $\tilde{\tilde{n}}$ & $\tilde{n}$ & $\underset{\exists}{\tilde{n}} \tilde{n}$ & $\cong$ \\
\hline 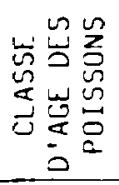 & $\begin{array}{l}\stackrel{c}{\Phi} \\
\overrightarrow{+}\end{array}$ & & & & 节 \\
\hline 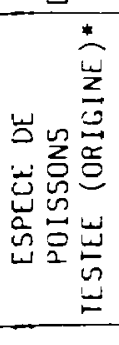 & 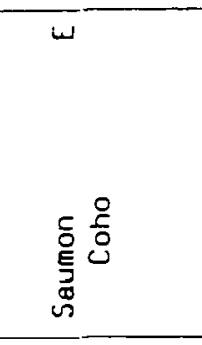 & 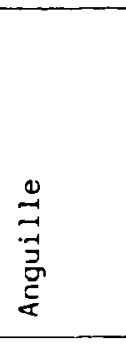 & 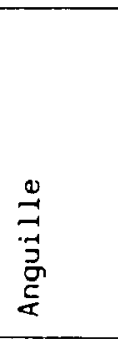 & 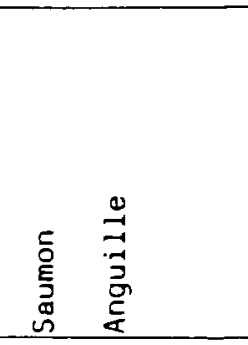 & 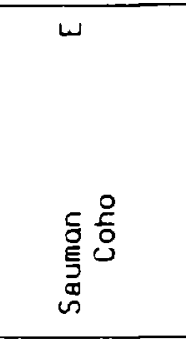 \\
\hline 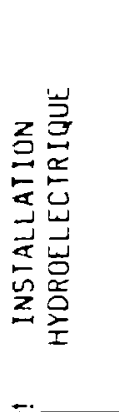 & 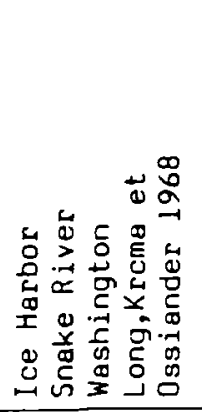 & 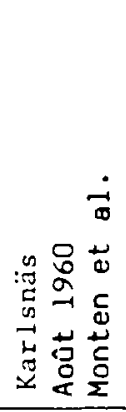 & 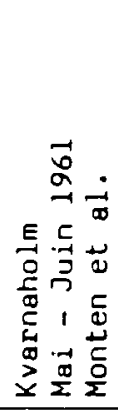 & 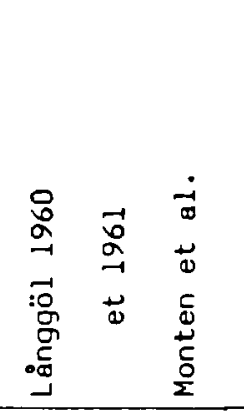 & 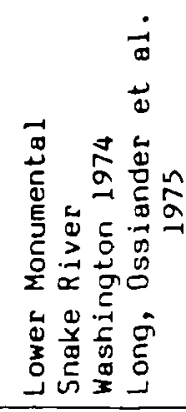 \\
\hline
\end{tabular}



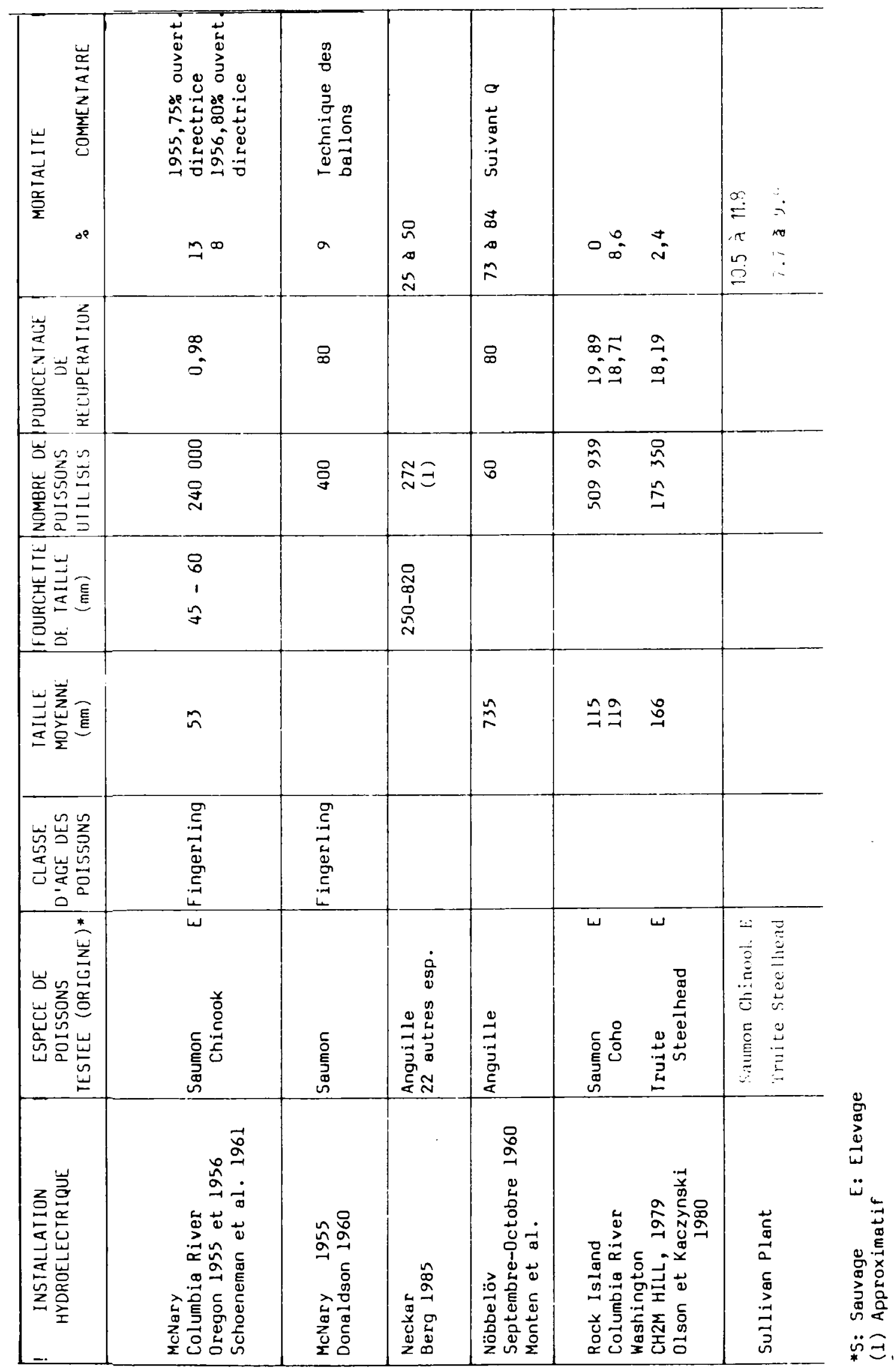


\begin{tabular}{|c|c|c|c|c|c|c|}
\hline 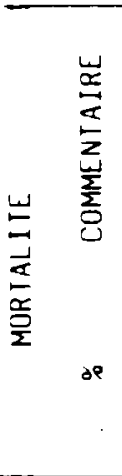 & 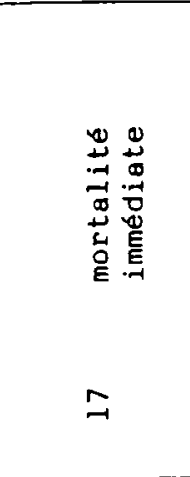 & 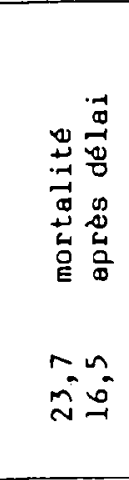 & 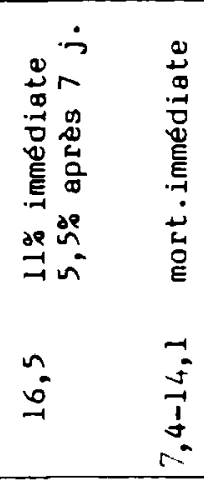 & 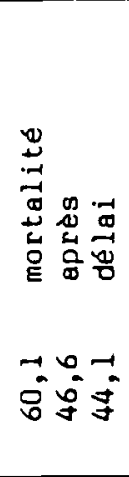 & 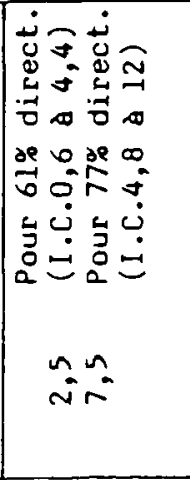 & 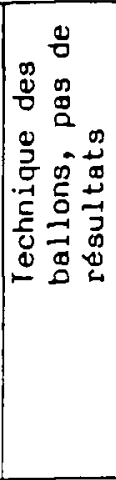 \\
\hline 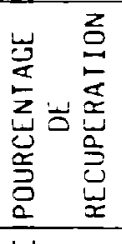 & 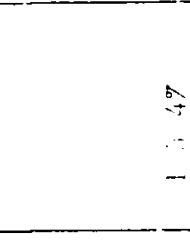 & & 今ั & $\stackrel{\Xi}{\Xi}$ & के & \\
\hline 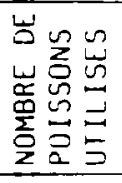 & 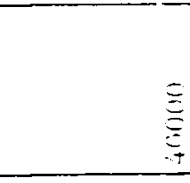 & & $\stackrel{g}{\Xi}$ & $\begin{array}{l}3 \\
\vdots \\
\vdots \\
=\end{array}$ & $\hat{\partial}$ & \\
\hline 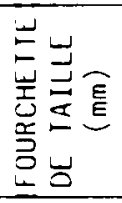 & $\begin{array}{l}9 \\
\underset{J}{9} \\
1 \\
\infty \\
\infty\end{array}$ & 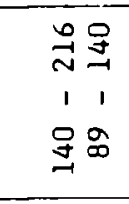 & 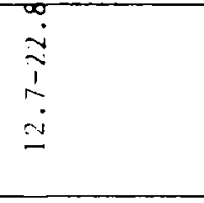 & & & \\
\hline 岂 & & & $\stackrel{\infty}{\infty} \quad \stackrel{\sim}{-1}$ & $\tilde{n} \Xi$ & & \\
\hline 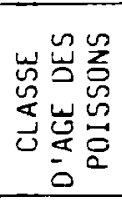 & 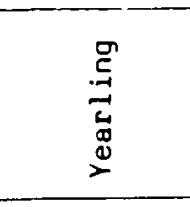 & 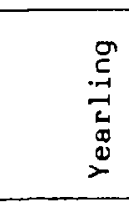 & $\begin{array}{c}\stackrel{g}{E} \\
\stackrel{\vec{G}}{G} \\
\stackrel{d}{2}\end{array}$ & $\begin{array}{l}5 \\
0 \\
0 \\
0 \\
\alpha \\
\alpha\end{array}$ & 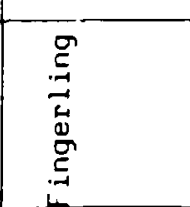 & \\
\hline 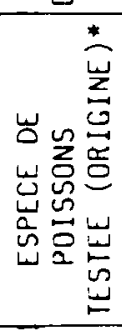 & 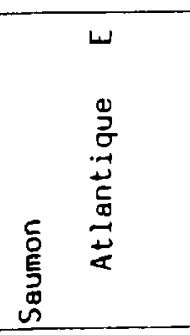 & 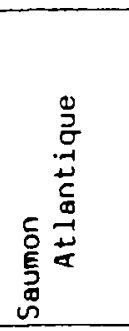 & 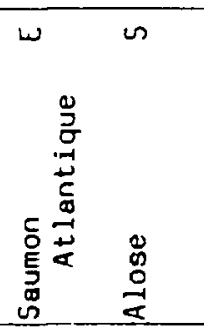 & $\underset{\stackrel{D}{2}}{\frac{D}{2}}$ & 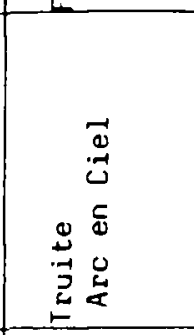 & \\
\hline 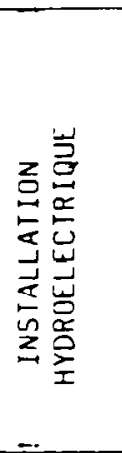 & 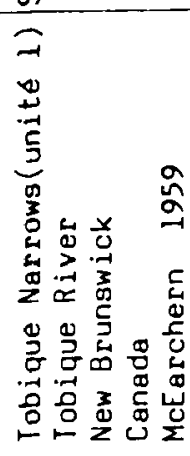 & 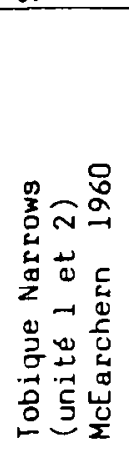 & 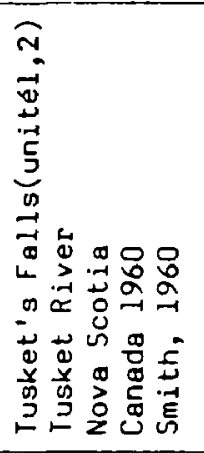 & 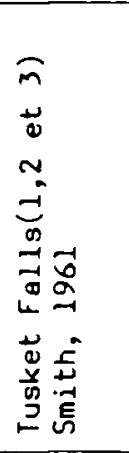 & 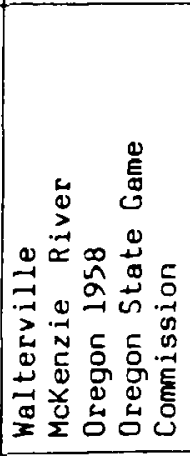 & 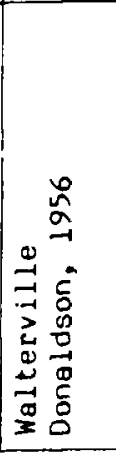 \\
\hline
\end{tabular}




\section{ANNEXE B \\ PRINCIPE DE LA DÉTERMINATION DES TRIANGLES DES VITESSES}

\section{Turbines FRANCIS}

Le principe de la détermination des triangles des vitesses est tiré de DERIAZ (in BROWN, 1958). On part des conditions de débit pour lesquelles la vitesse de l'eau en sortie de roue est axiale, c'est-à-dire sans composante périphérique. Ces conditions correspondent à un débit (noté Qax) légèrement supérieur au débit pour lequel le rendement de la turbine est maximal, débit noté Qopt (Qax = 1,04 Qopt)

Les diamètres d'entrée externes et internes de la roue (voir 1) seront notés respectivement par D1e et D1i, les diamètres de sortie de la roue externes et internes par D2e et D2i.

Les diamètres à mi-aubes en entrèe et en sortie seront notés D1m et D2m. D1m est très peu différent de D1i pour les turbines FRANCIS lentes, c'est-à-dire à faible vitesse spécifique.

La vitesse de rotation (en trs/mn) est notée $\mathrm{N}$, la chute nette (en mètres) $\mathrm{Hn}$.

La hauteur de l'aube en entrèe, peu differente de la hauteur des directrices pour les turbines à faibles vitesses spécifiques, sera notée $\mathrm{Be}$.

Dans les conditions d'écoulement axial en sortie de roue, on a :

$$
\mathrm{v} 2 \mathrm{u}=0
$$

En faisant l'hypothèse que la vitesse en sortie de roue est uniforme tout au long du bord de fuite de l'aube, on peut écrire:

$$
\mathrm{V} 2=\mathrm{V} 2 \mathrm{~m}=\mathrm{Qax} /\left(\mathrm{T} \cdot \mathrm{D} 2 \mathrm{e}^{2 / 4}\right)
$$

On détermine les caractéristiques du triangle des vitesses à mi-aube en sortie de roue :

$$
\begin{aligned}
\mathrm{U} 2 & =\tau . \mathrm{D} 2 \mathrm{~m} . \mathrm{N} / 60 \\
\mathrm{Bet} 2 & =\operatorname{arctg}(\mathrm{V} 2 \mathrm{~m} / \mathrm{U} 2) \\
\mathrm{Alf2}=90^{\circ} \quad & \text { (vitesse de sortie axiale) } \\
\mathrm{W} 2= & \text { I } \mathrm{V} 2 \mathrm{~m} / \mathrm{sin} \text { Bet2 I }
\end{aligned}
$$

On peut alors déterminer les caractéristiques du triangle d'entrée :

$$
\begin{gathered}
\mathrm{U} 1=\pi . D 1 \mathrm{~m} \cdot \mathrm{N} / 60 \\
\mathrm{~V} 1 \mathrm{~m}=\mathrm{Qax} / \pi \cdot \mathrm{D} 1 \mathrm{e} . \mathrm{Be} \\
\left.\mathrm{V} 1 \mathrm{u}=(\mathrm{m})^{\mathrm{n}} \cdot \mathrm{g} \cdot \mathrm{Hn}+\mathrm{U} 2 . \mathrm{V} 2 \mathrm{u}\right) / \mathrm{U} 1 \quad \text { avec } \mathrm{V} 2 \mathrm{u}=0
\end{gathered}
$$

$m^{h}$ est le rendement hydraulique défini dans la première partie. Il est plus grand de quelques pour cent - on prendra $2 \%$ - au rendement proprement dit de la turbine dans la mesure où sont prises en compte pour l'évaluation de ce dernier les pertes par fuite à travers les joints entre la roue et les parties fixes, les pertes par frottement aux paliers ainsi que les frottements au niveau de la bâche spirale et du distributeur.

$$
\begin{gathered}
V_{1}=\left(V_{1} m^{2}+V_{1} u^{2}\right)^{1 / 2} \\
\text { Bet1 }=90^{\circ}-\operatorname{arctg}\left(U_{1}-V_{1} u\right) / N_{1 m} \\
\text { Alf1 }=\arcsin \left(V_{1 m} / V_{1}\right) \\
W_{1}=\mid V_{1 m} / \sin \text { Bet1 I }
\end{gathered}
$$

On détermine ensuite les vitesses lorsque l'écoulement n'est plus axial en faisant l'hypothèse que l'angle Bet2 se conserve, d'où les relations:

$$
\begin{gathered}
\mathrm{v} 2 \mathrm{~m}=\mathrm{Q} /\left(\pi . \mathrm{D} 2 \mathrm{~s}^{2} / 4\right) \\
\mathrm{W} 2=\mid \mathrm{V} 2 \mathrm{msin} \text { Bet2 } \\
\mathrm{v} 2 \mathrm{u}=\mathrm{U} 2-\mathrm{W} 2 \cdot \cos B e t 2 \\
\mathrm{v} 2=\left(\mathrm{V} 2 \mathrm{~m}^{2}-\mathrm{V} 2 \mathrm{u}^{2}\right)^{1 / 2} \\
\mathrm{Alf2}=\arccos (\mathrm{V} 2 \mathrm{u} / \mathrm{N} 2)
\end{gathered}
$$

La détermination des triangles des vitesses en entrée s'effectue ensuite à l'aide des mêmes expressions que lorsque l'écoulement est axial, la seule différence étant que la composante $V 2 u$ n'est plus nulle:

$$
\begin{gathered}
\mathrm{V} 1 \mathrm{~m}=\mathrm{Q} / \pi \cdot \mathrm{D} 1 \mathrm{e} \cdot \mathrm{Be} \\
\mathrm{V} 1 \mathrm{u}=\left(\mathrm{m}^{\mathrm{h}} \cdot \mathrm{g} \cdot \mathrm{Hn}+\mathrm{U} 2 . \mathrm{V} 2 \mathrm{u}\right) / \mathrm{U} 1 \\
\mathrm{~V} 1=\left(\mathrm{V} 1 \mathrm{~m}^{2}-\mathrm{V}_{1} \mathrm{u}^{2}\right)^{1 / 2} \\
\text { Bet1 }=90^{\circ}-\operatorname{arctg}(\mathrm{U} 1-\mathrm{V} 1 \mathrm{u}) / \mathrm{V} 1 \mathrm{~m} \\
\text { Alf1 }=\arcsin (\mathrm{V} 1 \mathrm{~m} / \mathrm{V} 1)
\end{gathered}
$$


Exemple : cas d'une turbine FRANCIS tournant à $\mathrm{N}=214 \mathrm{trs} / \mathrm{mn}$ sous $\mathrm{Hn}=79 \mathrm{~m}$ de chute nette, débit aux conditions de rendement maximum (.895) de $53 \mathrm{~m}^{3} / \mathrm{s}$. Diamètres (externes, internes) en entrée et en sortie : $3,0 \mathrm{~m}, 2,68 \mathrm{~m}, 2,81 \mathrm{~m}, 1,17 \mathrm{~m}$.

Qax $=56 \mathrm{~m}^{3} / \mathrm{s}$

Conditions axiales:

$\begin{array}{ll}\mathrm{v} 2 \mathrm{u}=0 & \mathrm{v} 2=\mathrm{V} 2 \mathrm{~m}=8.65 \mathrm{~m} / \mathrm{s} \\ \mathrm{U} 2=22.4 \mathrm{~m} / \mathrm{s} & \text { Bet2 }=21^{\circ} \\ \mathrm{W} 2=24 \mathrm{~m} / \mathrm{s} & \mathrm{U}_{1}=30,4 \mathrm{~m} / \mathrm{s} \\ \mathrm{V} 1 \mathrm{u}=23,3 \mathrm{~m} / \mathrm{s} & \mathrm{V} 1=24,7 \mathrm{~m} / \mathrm{s} \\ \text { Alf1 }=19,4^{\circ} & \mathrm{W}_{1}=6.1 \mathrm{~m} / \mathrm{s}\end{array}$

$$
\begin{aligned}
& \text { Alf2 }=90^{\circ} \\
& \text { v1m }=8,2 \mathrm{~m} / \mathrm{s} \\
& \text { Bet } 1=49,2^{\circ}
\end{aligned}
$$

Conditions optimales ( $3 / 4$ charge) :

$\mathrm{Q}=53 \mathrm{~m}^{3} / \mathrm{s}$, rendement hydraulique : .915

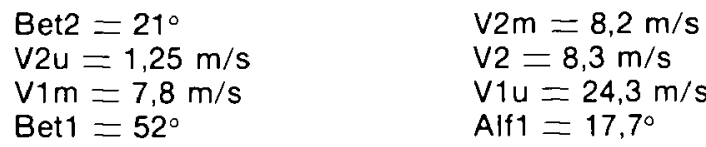

\begin{tabular}{|c|c|c|}
\hline & & \\
\hline Bet2 $=21^{\circ}$ & $\mathrm{v} 2 \mathrm{~m}=10,6 \mathrm{~m} / \mathrm{s}$ & $W 2=29,6 \mathrm{~m} / \mathrm{s}$ \\
\hline $\mathrm{v} 2 \mathrm{u}=5,2 \mathrm{~m} / \mathrm{s}$ & $\mathrm{V} 2=11,9 \mathrm{ll} / \mathrm{s}$ & Alf2 $=116^{\circ}$ \\
\hline $\mathrm{v} 1 \mathrm{~m}=10,1 \mathrm{~m} / \mathrm{s}$ & $v 1 u=18 \mathrm{~m} / \mathrm{s}$ & $v_{1}=20,7 \mathrm{~m} / \mathrm{s}$ \\
\hline Bet1 $=39^{\circ}$ & Alf1 $=29,3^{\circ}$ & $W_{1}=15,9 \mathrm{~m} / \mathrm{s}$ \\
\hline Demi-chars & & \\
\hline $\mathrm{Q}=37 \mathrm{~m}^{3} / \mathrm{s}$, ren & aulique : .83 & \\
\hline Bet2 $=21^{\circ}$ & $v 2 \mathrm{~m}=5,7 \mathrm{~m} / \mathrm{s}$ & $\mathrm{W} 2=15,7 \mathrm{~m} / \mathrm{s}$ \\
\hline $\mathrm{v} 2 \mathrm{u}=7,7 \mathrm{~m} / \mathrm{s}$ & $\mathrm{v} 2=9,6 \mathrm{~m} / \mathrm{s}$ & Alf2 $=36^{\circ}$ \\
\hline $\mathrm{v} 1 \mathrm{~m}=5,4 \mathrm{~m} / \mathrm{s}$ & v1u $=27,5 \mathrm{~m} / \mathrm{s}$ & $v_{1}=28 \mathrm{~m} / \mathrm{s}$ \\
\hline Bet $1=61^{\circ}$ & Alf1 $=11^{\circ}$ & $W_{1}=6.1 \mathrm{~m} / \mathrm{s}$ \\
\hline
\end{tabular}

Conditions maximales:

$\mathrm{Q}=69 \mathrm{~m}^{3 / \mathrm{s}}$, rendement hydraulique : .835

\section{Turbines KAPLAN}

Le principe de calcul des vitesses en entrée et en sortie de roue pour un débit donné est très voisin de celui utilisé pour les turbines FRANCIS. On commence à déterminer les triangles des vitesses dans les conditions de sortie axiales (c'est-à-dire avec une composante giratoire de la vitesse de sortie V2u nulle) pour un débit légèrement supérieur au débit en question. Les triangles des vitesses sont dans un premier temps calculés à la périphérie. On passe ensuite aux conditions recherchées en écrivant que l'angle Bet2 se conserve. On passe ensuite de la périphérie au moyeu ou à une position quelconque intermédiaire en écrivant que l'écoulement est sans échange d'ènergie et suit la loi du vortex libre.

On notera par $\mathrm{Dp}, \mathrm{Dc}$ et $\mathrm{Dm}$ les diamètres de la roue à la périphérie, au moyeu et à mi-pale. Les vitesses et les angles seront indicés par $p, c$ et $m$ suivant qu'ils sont relatifs à la périphérie, au moyeu ou à mi-pale.

Pour les conditions axiales, c'est-à-dire un débit légèrement supérieur au débit $Q$ considèré, à la périphérie de la roue:

$$
\begin{aligned}
& \mathrm{U} 1 \mathrm{p}=\mathrm{U} 2 \mathrm{p}=\boldsymbol{\tau} \cdot \mathrm{Dp} \cdot \mathrm{N} / 60 \\
& \mathrm{v} 1 \mathrm{mp}=\mathrm{V} 2 \mathrm{mp}=\mathrm{V} 1 \mathrm{~m}=\mathrm{Qax} /\left(\mathrm{T} \cdot \mathrm{Dp} \mathrm{p}^{2} / 4\right) \\
& \mathrm{V} 2 \mathrm{up}=0 \\
& \text { Alf2p }=0 \\
& \text { Bet2p }=\operatorname{arctg}\left(V_{1 m} / U_{1 p}\right) \\
& W_{2} p=V 1 \mathrm{~m} / \mathrm{sin} \text { Bet } 2 p \\
& \text { V1up }=\left(m^{n} \cdot g \cdot H n+U 1 \cdot V 2 u\right) / U 1 p \quad \text { avec } V_{2} u p=0 \\
& V_{1 p}=\left(V_{1} m^{2}+V_{1 u p^{2}}\right)^{1 / 2} \\
& \text { Bet } 1 p=90^{\circ}-\operatorname{arctg}(U 1-V 1 u p) / V 1 m \\
& W 1 p=\mid V 1 \mathrm{~m} / \mathrm{sin} \text { Bet } 1 p \mid \\
& \text { Alf1p }=\arcsin (\mathrm{V} 1 \mathrm{~m} / \mathrm{V} 1 \mathrm{p})
\end{aligned}
$$

Pour les conditions recherchées (débit $Q$ ), l'angle Bet2p se conserve :

$$
\begin{gathered}
V 1 \mathrm{~m}=\mathrm{Q} /\left(\tau . D \mathrm{p}^{2 / 4)}\right. \\
W 2 \mathrm{p}=\mid \mathrm{V} 1 \mathrm{~m} / \mathrm{sin} \text { Bet } 2 \mathrm{p} \mid
\end{gathered}
$$




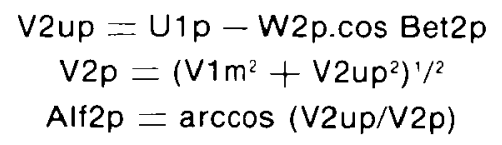

Les expressions permettant de calculer le triangle des vitesses en entrée sont identiques à celles utilisées pour les conditions axiales:

$$
\begin{aligned}
& \text { V1up }=1 / U 1 p\left(m^{n} \cdot g \cdot H n+U 1 p \cdot V 2 u p\right) \\
& V 1 p=\left(V_{1} m^{2}+V 1 u p^{2}\right)^{1 / 2} \\
& \text { Bet1p }=90^{\circ}-\operatorname{arctg}(\mathrm{U} 1 \mathrm{P}-\mathrm{V} 1 \mathrm{up}) / \mathrm{V} 1 \mathrm{~m} \\
& W 1 \mathrm{p}=\mid \mathrm{V} 1 \mathrm{~m} / \mathrm{sin} \text { Bet } 1 \mathrm{p} \mid \\
& \text { Alf1p }=\arcsin (V 1 m / V 1 p)
\end{aligned}
$$

Les relations suivantes permettent de passer des triangles des vitesses à la périphérie à ceux au moyeu (ou à ceux afférents à un diamètre quelconque, en particulier au diamètre à mi-pale) :

$$
\begin{gathered}
V 2 u c=(D p / D c) . V 2 u p \\
V 1 u c=(D p / D c) \cdot V 1 u p \\
U 1 c=(D c / D p) . U 1 p \\
V v 2 m p=V 1 m c=V 2 m c=V 1 m \\
V 1 c=\left(V 1 u c^{2}+V 1 m^{2}\right)^{1 / 2} \\
V 2 c=\left(V 2 u c^{2}+V 1 m^{2}\right)^{1 / 2} \\
W 1 c=\left((U 1 c-V 1 u c)^{2}+V 1 m^{2}\right)^{1 / 2} \\
W 2 c=\left((U 1 c-V 2 u c)^{2}+V 1 m^{2}\right)^{1 / 2} \\
\text { Alf1c }=\arccos (V 1 u c / V 1 c) \\
\text { Alf2c }=\arccos (V 2 u c / V 2 c) \\
\text { Bet1c }=\arcsin (V 1 m / W 1) \\
\text { Bet2c }=\arcsin (V 1 m / W 2)
\end{gathered}
$$




\section{ANNEXE C}

\section{IDENTIFICATION DES TESTS}

\section{TURBINES FRANCIS}

Installation

Abréviation

No test

Couzon

COUZ

Poutès

POUT

Mauzac

MAUZ

Stornorrfors

STORN

UPHEM

MOTA

Motala

CUSH

Shasta SHAS

Glines GLIN

Baker

BAKER

SETON

ELWHA

1 à 9

10 à 12

13 à 14

15 à 17

18 à 23

24 à 29

30 à 42

43 à 55

56 à 57

58

59

Elwha

PUNT

60

Puntledge

LEQ

61 à 64

65 à 85

\section{TURBINES KAPLAN ET BULBES - SALMONIDÉS}

\begin{tabular}{ll}
\multicolumn{1}{c}{ Installation } & Abréviation \\
Laïlnacar & OLOR \\
Tuilières & TUIL \\
Angabäck & ANG \\
Langgöl & LAG \\
Motala & MOT \\
Rock Island & ROC \\
Essex & ESS \\
Foster & FOS \\
Big Cliff & BIG \\
MacNary & MCN \\
Tobique & TOB \\
Tusket & TUS \\
Holyoke & HOLY \\
Lower Monumental & LOW
\end{tabular}

No test

1 à 3

4 à 7

7 à 10

11 à 12

13 à 19

20 à 22

23 à 24

25 à 37

38 à 50

51 à 52

53 à 54

55

56

57 à 58

\section{TURBINES KAPLAN ET BULBES - ANGUILLES}

Installation

Kvarnaholm

Broby

Nöbbelöv

Emsfors

Karlnäs

Gustavsberg

Langgöl

Angabäck
Abreviation

\section{EKV}

EBR

ENO

EEM

EKA

EGU

ELA

EAN
No test

1 a 3

4 à 5

6 à 7

8 à 9

10 à 11

12 à 15

16 à 19

20 à 22 

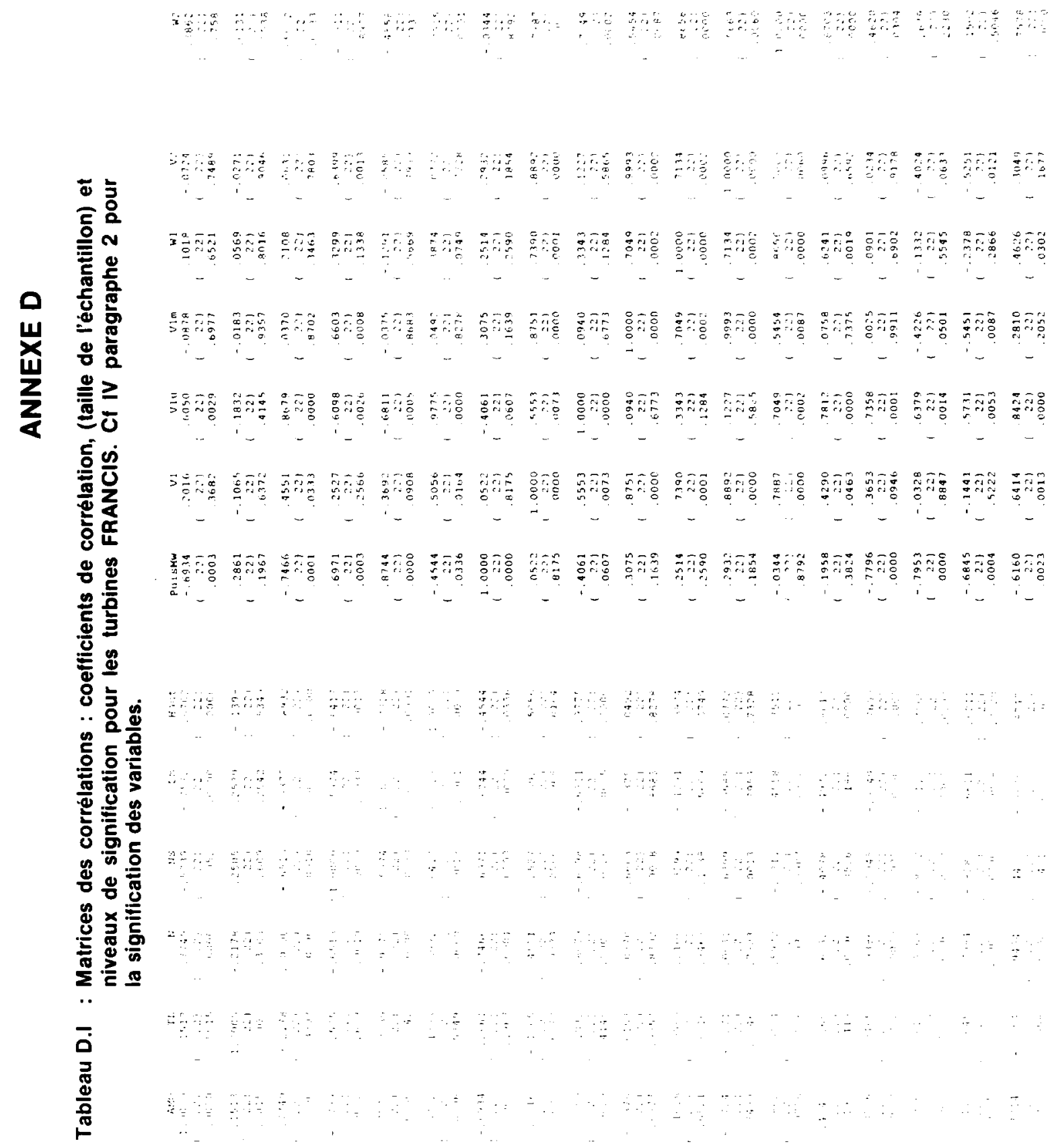


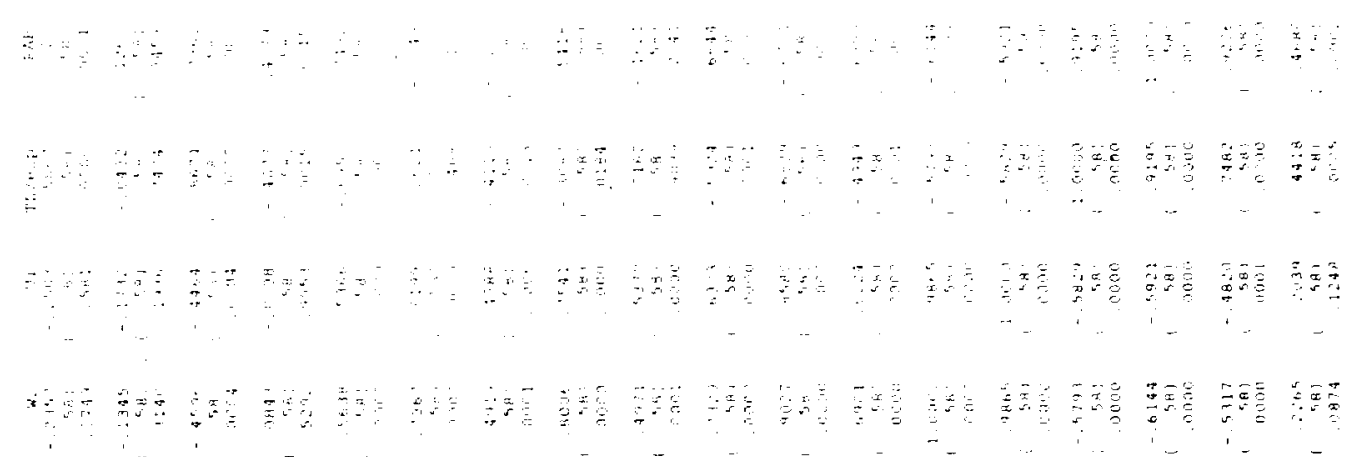

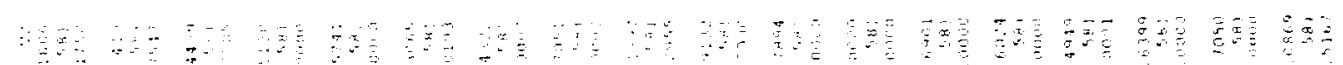

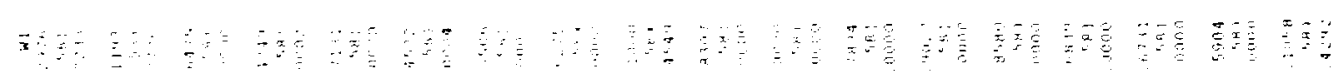

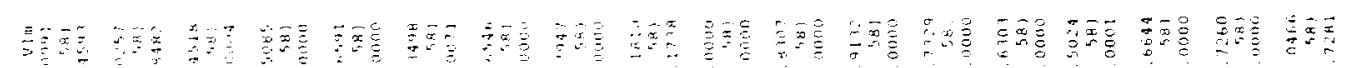

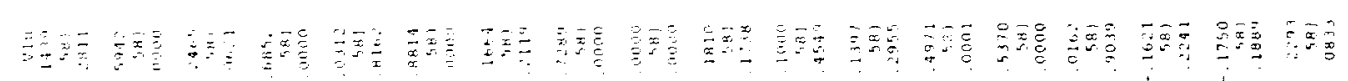

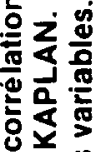

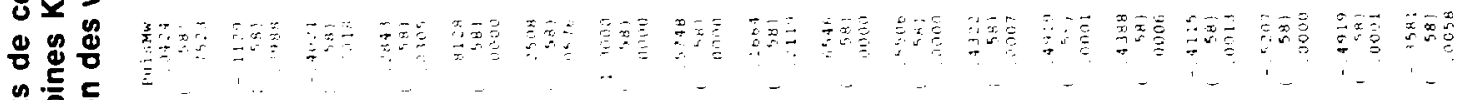
 
Bull. Fr. Pêche Piscic. (1989) 312 - 313

$-86-$

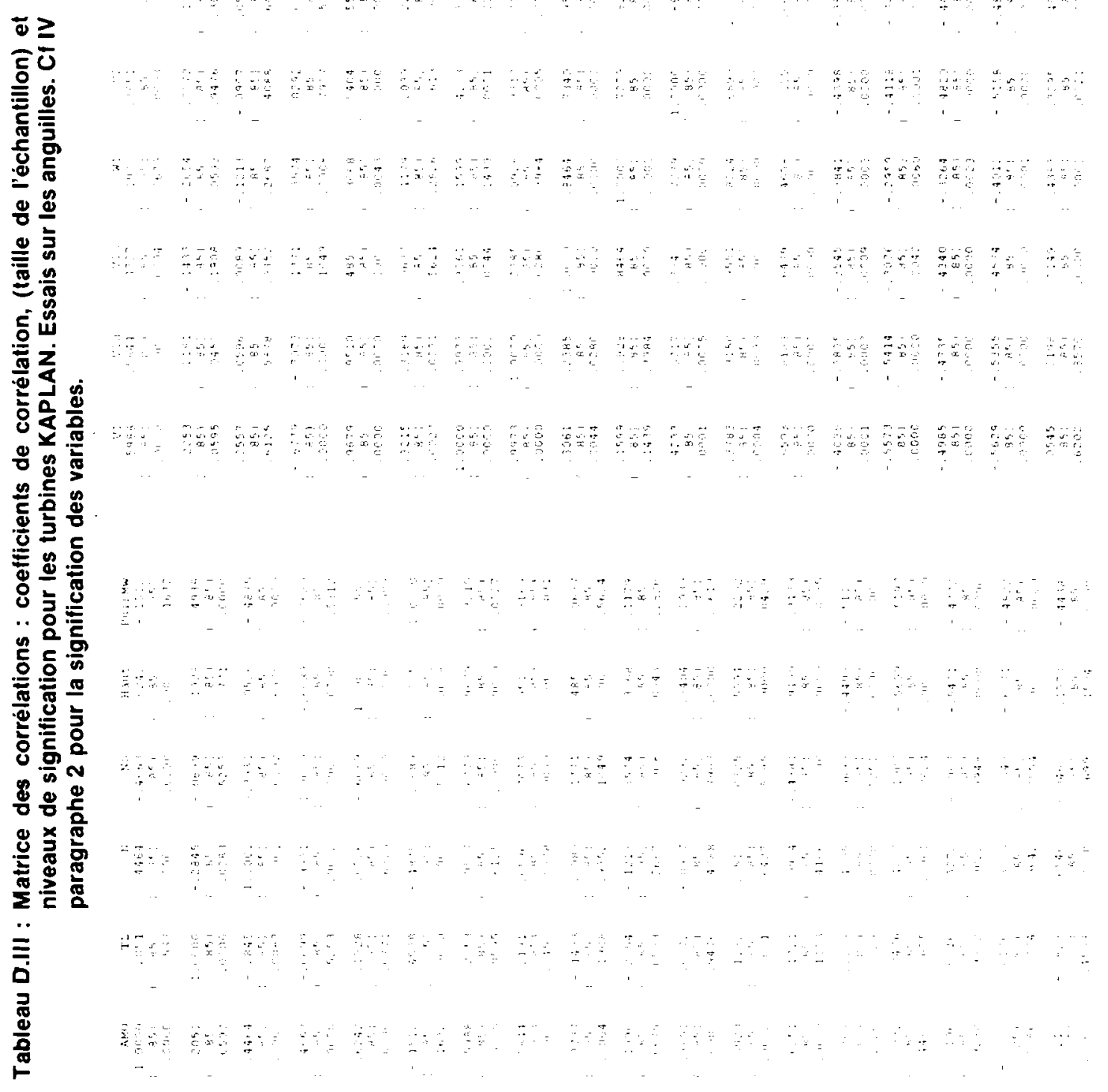


Bull. Fr. Pèche Piscic. (1989) $312-313$

$-87-$

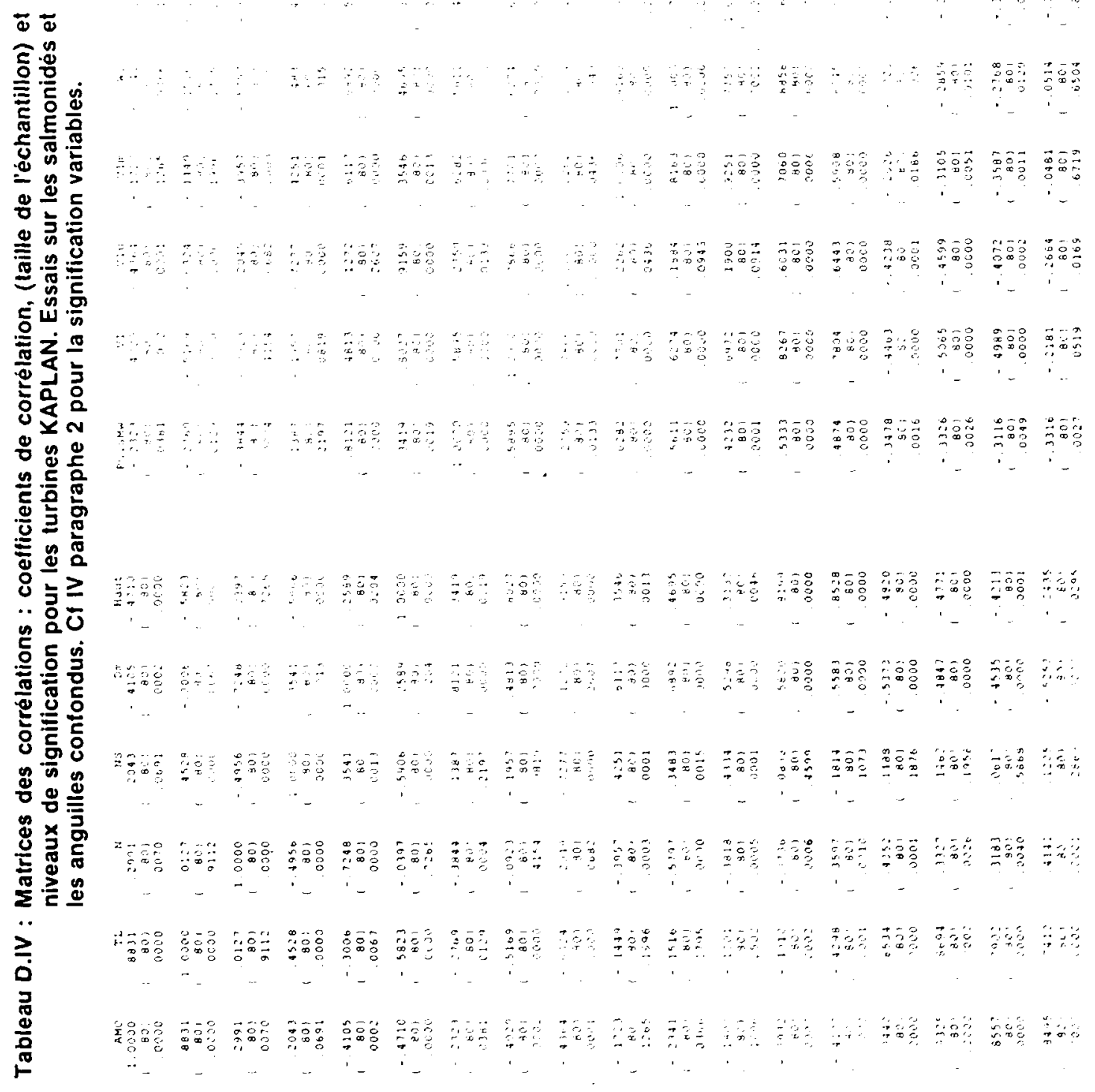




\section{BIBLIOGRAPHIE}

ANDREW F.J., GEEN G.H., 1958. Sockeye and pink Salmon investigations at the Seton Creek hydroelectric installation. Progress report. International Pacific Salmon Fisheries Commission, New Wesminster, B.C., 74 p.

BELL M.C., DELACY A.C., PAULIK G.J., 1967. A compendium on the success of passage of small fish through turbines. U.S. Army Corps of Engineers. North Pacific Division. Fisheries Engineering Research Program, Portland, Oregon, $204 \mathrm{p}$.

BELL M.C., BRUYA K.J., 1981. Updated compendium on the success of passage of small fish through turbines. U.S. Army Corps of Engineers, North Pacific Division, Fisheries Engineering Program, Portland, Oregon, $91 \mathrm{p}$.

BELL C.E., KYNARD B., 1985. Mortality of adult shad passing through a $17 \mathrm{MW}$ Kaplan turbine at a low-head hydroelectric dam. North Am. J. Fish. Management, 5, 33-38.

BERG R., 1985. Investigation on injuries of migrating eels caused by Kaplan turbines. Working party on Eel organized by C.E.C.P.I. (F.A.O.), Perpignan, $13 p$.

BEYER D.L., D'AOUST B.G., SMITH L.S., 1976. Decompression induced bubble disease. Under sea Biomedical Research, 3, 321-338.

BROWN J.G., 1958. Hydroelectric engineering practice. Vol II, Blackie and son Ltd, London, $740 \mathrm{p}$.

CRAMER F.W., DONALDSON I.J., 1964. Evolution of recovery nets used in tests on fish passage through hydraulics turbines. Prog. Fish. Cult., 26, 36-41.

CRAMER F.W., OLIGHER R.C., 1964. Passing fish through hydraulic turbines. Trans. Am. Fish. SOC., 93 (3), 243-259.

DARTIGUELONGUE J., LARINIER M., 1987. Evaluation des dommages subis par les juveniles de salmonidés lors de leur passage à travers les turbines de microcentrales de St-Pée-surNivelle et Laïlhacar (Pyrénées Atlantiques). in THIBAULT M. et BILLARD R., La restauration des rivières à saumons, $175-182$ I.N.R.A., Paris.

DARTIGUELONGUE J., LARINIER M., 1987. Mise au point d'un protocole expérimental pour l'évaluation des dommages subis par les juvéniles lors de leur transit à travers des turbines. Rapport CEMAGREF convention DPN 85/8, 42 p.

DARTIGUELONGUE J., 1988. Contribution à l'étude de la mortalité des poissons au passage des turbines d'installations hydroélectriques/méthodologie et analyse des données. Thèse de doctorat, I.N.P. Toulouse, 200 p.

EICHER ASSOCIATES INC., 1987. Turbine-related fish mortality: review and evaluation of studies. E.P.R.I. Research project 2694-4. Final report, 102 p.

FEATHERS M.G., KNABLE A.E., 1983. Effects of depressurization upon largemouth Bass. North Am. J. Fish. Management, 3, 86-90.

GINOCCHIO R., 1978. L'énergie hydraulique. Collection de la direction des études et recherches d'Électricité de France. Eyrolles ed., Paris, $598 \mathrm{p}$.

GLOSS S.P., WALH J.R., 1983. Mortality of juvenile salmonids passing through Ossberger crossflow turbines at small-scale hydroelectric sites. Trans. Am. Fish. Soc., 112, 194-200.

GLOSS S.P., WALH J.R., DUBOIS R.B., 1982. Potential effects of ossberger turbine on Atlantic Salmon smolts, Striped Bass and American Shad, in Potential effect of Kaplan, Ossberger and Bulb turbines on anadromous fishes of the Northeast United States. Final technical report, FWS/OBS-82/62, 51-90, Fish and Wildlife Service, U.S. Dept. of the Int., Newton Corner, MASS.

GROVES A.B., 1972. Effects of hydraulic shearing action on juvenile salmon. in U.S. Army Corps of Engineers, North Pacific Division, 1976. Fourth Progress Report on Fisheries Engineering Research Program, $670 \mathrm{p}$.

HAMILTON J.A.R., ANDREW F.J., 1954. An investigation of the effect of Baker dam on downstreammigrant salmon. Internat. Pac. Salmon Fish. Comm. Bull. 6, New Westminster, B.C., 73 p.

JONES F.R.H., 1951. The swimmbladder and the vertical movenents of the teleostean fishes. I. Physical factors. J. Exp. Biol., 28, 553-566.

JONES F.R.H., 1952. The swimmbladder and the vertical movement of the teleostean fishes. II. The restriction to the rapid and slow movements. J. Exp. Biol., 29, 94-109.

JOHNSON R.L., 1970. Fingerling fish mortalities at 57.5 fps. in Fourth Progress Report on Fisheries Research Program 1966-1972. 355-370. U.S. Army Corps of Engineers, North Pacific Division, Portland Oregon.

JOHNSON R.L., 1970. Fingerling fish research effect of mortality of 67 fps velocity. in Fourth Progress Report on Fisheries Research Program 1966-1972. 371-378. U.S. Army Corps of Engineers, North Pacific Division, Portland Oregon. 
JOHNSON R.L., 1972. Fingerling fish research, high-velocity flow through four-inch nozzle. in Fourth Progress Report on Fisheries Research Program 1966-1972. 379-383. U.S. Army Corps of Engineers, North Pacific Division, Portland Oregon.

KNIGHT A.E., KUSMESKUS D., 1982. Potential effect of bulbs turbines on Atlantic Salmon, in Potential effect of Kaplan, Ossberger and Bulb turbines on anadromous fishes of the Northeast United States, Final Technical report, FWS/OBS-82/62, 91-98, Fish and Wildlife Service, U.S. Dept. of the Int., Newton Corner, MASS.

KYNARD B., TAYLOR R., BELL C., STIER D., 1982. Potential effects of Kaplan turbines on Atlantic Salmon smolts, American shad and Blueback herring, in Potential effect of Kaplan, Ossberger and Bulb turbines on anadromous fishes of the Northeast United States, Final technical report, FWS/OBS-82/62, 5-50, Fish an Wildlife Service, U.S. Dept. of the Int., Newton Corner, MASS.

KREITMANN L., 1931. Le franchissement des installations hydroélectriques par les poissons. Congreso Intern. Oceanografia, hidrografia, marina y hidrologia continental, Seville. 30 p.

LAMPERT W., 1976. Experiments on the resistance os fish to rapid increase in hydrostatic pressure. J. Fish. Biol. 8, 381-383.

LONG C.W., KROMA R.F., OSSIANDER F.J., 1968. Research on fingerling mortality in Kaplan turbines. U.S. Bureau of Commercial Fisheries, Biological Laboratory, Seattle WA., 7 p.

LONG C.W., OSSIANDER F.J., RUEHLE T.E., MATTHEWS G.M., 1975. Survival of coho salmon fingerlings passing through operating turbines with and without perforated bulkheads and of steelhead trout fingerlings passing through spillways with and without a flow deflector. Nat. Marine Fish. Serv., Coastal Zone and estuarine Studies, Northwest Fisheries Center, Seattle, Final report, $7 \mathrm{p}$.

LUCAS K.C., 1962. The mortality to fish passing through hydraulic turbines as related to cavitation and performance characteristics, pressure change, negative pressure and other factors, in Cavitation and hydraulic machinery, Proceedings of I.A.R.H. Symposium, 307-335, NUMACHI editor, Sensaï, Japan.

MC EACHERN N., 1960. Mortality tests at Tobique Narrows dam. Canada Department of Fisheries, Halifax N.S., Report 60-12, 11 p.

MONTEN E., 1985. Fish and turbines. Fish injuries during passage through power station turbines. Vattenfall, Stockholm, $111 \mathrm{p}$.

MONTREAL ENGINEERING COMPANY, Ltd, 1981. Fish mortality as a function of the hydraulic properties of turbines. Canadian Electrical Association, Research and Development, report G 144, 75 p.

MONTREAL ENGINEERING COMPANY Ltd, 1982. Fish mortality in Francis turbines. Canadian Electrical Association, Research and Development, report $G$ 261, $131 p$.

MUIR S.F., 1959. Passage of young fish through turbines. J. Power Division, Proc. A.S.C.E., 85, 23-46.

MUNRO W.R., 1965. Effect of the passage through hydroelectric turbines on salmonids. I.C.E.S., Salmon and Trout Committee, 57,5 p.

OLIGHER R.C., DONALDSON I.J., 1966. Fish passage through turbines: tests at Big Cliff hydroelectric plant. Progress report $n^{\circ} 6$, U.S. Army Corps of Engineers, Walla Walla District.

OLSON F.W., KACZINSKI V.W., 1980. Survival of downstream migrant coho salmon and steelhead trout through Bulb turbines. Public Utility District no 1 of Chelan County, Wenatchee, WA., $43 p$.

OTTERSTROM C.V., 1931. Die Turbinen und die Abwärts wandernden jungen Lachse und Forellen. J. Cons. Internat. Expl. Mer, vol VI, no 2, 273-286.

OTTERSTROM C.V., 1932. Die Turbinen und die Abwäts wandernden jungen Lachse und Forellen. Teil 2. J. Cons. Internat. Expl. Mer, vol VII, n 1, 63-75.

PYPER J. 1958. Pressure test on sockeye smolts. Intern. Pacific Salmon Fish. Comm., unpublished report, $6 \mathrm{p}$.

PYPER J., HARVEY H.H., JOHNSTON L.W., 1958. Adult sockeye pressure studies. Cultus Lake, B.C. Intern. Pacific Salmon Fish. Comm., unpublished report, $14 \mathrm{p}$.

ROWLEY W.E., 1955. Hydrostatic pressure tests on rainbow trout. California Fish and Game, Vol. $41, n^{\circ} 3,243-244$.

RUGGLES C.P., 1980. A review of the downstream migration of Atlantic Salmon. Can. Tech. Rep. of Fish. and Aqua. Sciences $n^{\circ} 952,37 \mathrm{p}$.

SCHOENEMAN D.E., JUNGE C.O., 1954. Investigations of mortalities to downstream migrant salmon at two dams on the Elwha river. Washington State Department of Fisheries. Research Bulletin $n^{\circ} 3,1954,51 \mathrm{p}$.

SCHOENEMAN D.E., PRESSEY R.T., JUNGE C.O., 1961. Mortalities migrant salmon at McNary dam. Trans. Am. Fish. Soc., 90, 58-72. 
SEDILLE M., 1967. Turbomachines, machines hydrauliques et thermiques. Tome II, Masson, Paris, $572 \mathrm{p}$.

SEMPLE J.R., 1979. Downstream migration facilities and turbine mortality evaluation, Atlantic Salmon smolts at Malays Falls, Nova Scotia. Fisheries and Marine Service Manuscript Report $n^{\circ} 1541$, Department of Fisheries and Oceans, Halifax, Nova Scotia, $15 p$.

SMITH K.E.H., 1960. Mortality tests-young salmon and gaspereau at Tusket River power dam, Yarmouth County. Canada Dept. of Fisheries, Halifax, Nova Scotia, report 60-13, 18 p.

SMITH K.E.H., 1961. Mortality tests-yearling gaspereau at Tusket River power dam, Yarmouth County. Canada Dept. of Fisheries, Halifax, Nova Scotia, report 61-9, 19 p.

STIER D.J., KYNARD B., 1986. Use of radio telemetry to determine the mortality of Atlantic Salmon smolts passed through a $17 \mathrm{MW}$ Kaplan turbine at a low-head hydroelectric dam. Trans. Am. Fish. Soc., 115, 771-775.

TAYLOR R.E., KYNARD B., 1985. Mortality of juvenile american shad and blueback herring passed through a low-head Kaplan hydroelectric turbine. Trans. Am. Fish. Soc., 114, 430-435.

TOMASSONE R., LESQUOI E., MILLIER C., 1983. La régression. Nouveaux regards sur une ancienne méthode statistique. Masson, Paris, $180 \mathrm{p}$.

TRAVADE F., DARTIGUELONGUE J., LARINIER M., 1987. Dévalaison et franchissement des turbines et ouvrages énergétiques : expériences E.D.F.. La Houille Blanche, $n^{\circ} 1 / 2,125-133$.

TSVETKOV V.I., PAVLOV D.S., NEZDOLIY V.K., 1971. Changes of hydrostatic pressure lethal to the young of some freshwater fish. J. Ichthiol., 12, 307-318.

TURBAK S.C., REICHE D.R., SHRIMER C.R., 1981. Analysis of environmental issues related to small-scale hydroelectric development. IV : Fish mortality resulting from turbine passage. ORNL/TM-7521. Oak Ridge National Laboratory, Oak Ridge, Tennessee, $116 \mathrm{p}$.

U.S. ARMY CORPS OF ENGINEERS, North Pacific Division, 1956. First Progress Report on Fisheries Engineering Research Program, $179 \mathrm{p}$.

U.S. ARMY CORPS OF ENGINEERS, North Pacific Division, 1960. Second Progress Report on Fisheries Engineering Research Program, $152 \mathrm{p}$.

U.S. ARMY CORPS OF ENGINEERS, North Pacific Division, 1966. Third Progress Report on Fisheries Engineering Research Program, $124 \mathrm{p}$.

U.S. ARMY CORPS OF ENGINEERS, North Pacific Division, 1976. Fourth Progress Report on Fisheries Engineering Research Program, $670 \mathrm{p}$.

U.S. ARMY CORPS OF ENGINEERS, North Pacific Division, 1979. Fifth Progress Report on Fisheries Engineering Research Program, $530 \mathrm{p}$.

U.S. ARMY CORPS OF ENGINEERS, Walla Walla district, 1963. Fish passage through turbines tests at Shasta hydroelectric plant. Progress report $n^{\circ} 5$, Walla Walla WA., $18 \mathrm{p}$.

VIVIER L., 1966. Turbines hydrauliques et leur régulation. Théorie, construction utilisation. Albin Michel, Paris, 495 p.

VON GUNTEN G.H., 1961. Fish passage through hydraulic turbines. J. Power Division, Proc A.S.C.E., Vol. $87,59-72$. 